Prepared in cooperation with the California State Water Resources Control Board

\title{
Groundwater-Quality Data for the Madera/Chowchilla- Kings Shallow Aquifer Study Unit, 2013-14: Results from the California GAMA Program
}

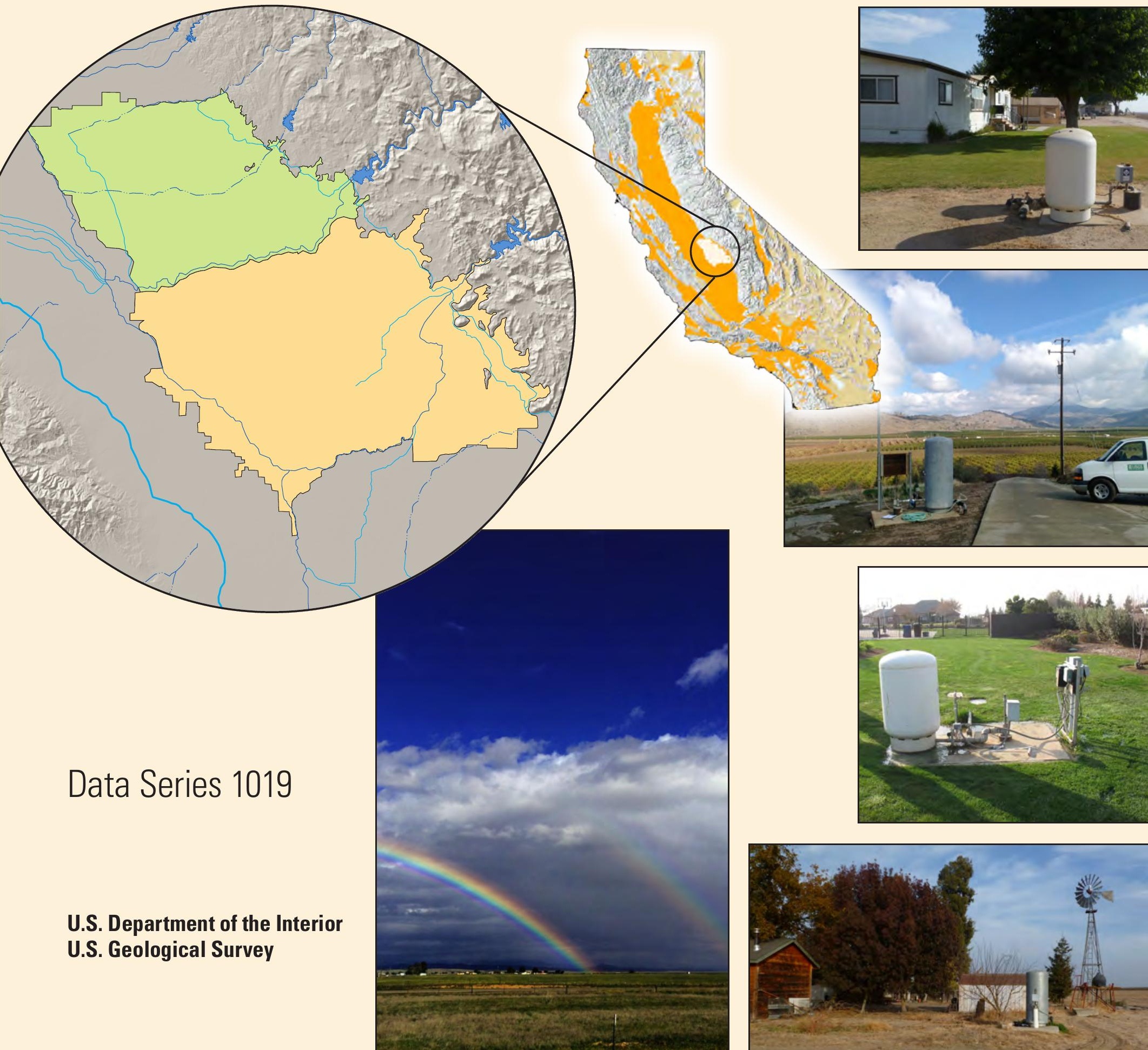


Cover photographs

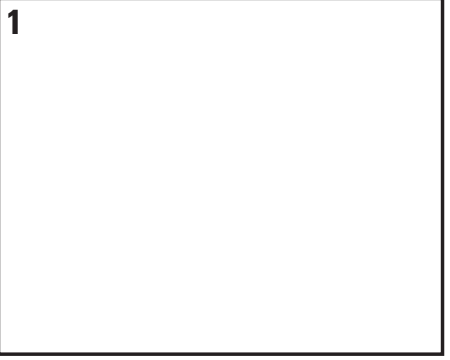

2

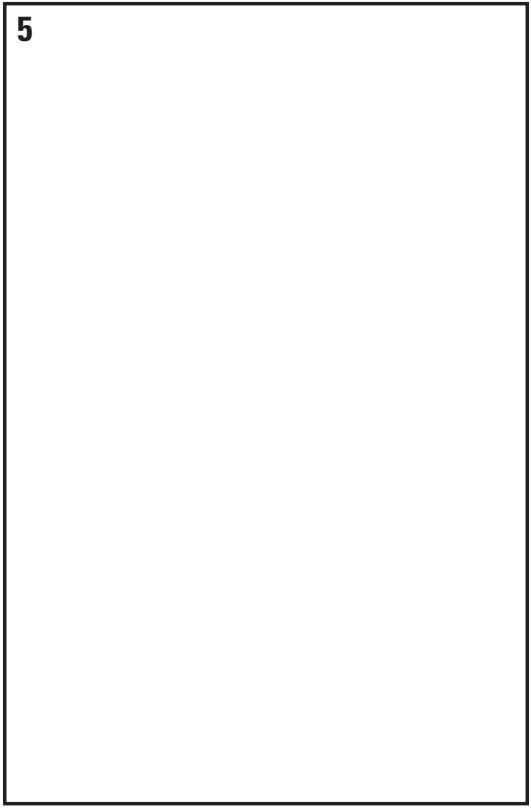

3

4

Cover. 1. Well near El Nido, California. (Photograph taken by Antonia J. Salas, U.S. Geological Survey.)

2. Well near Orange Cove, California. (Photograph taken by Jennifer L. Shelton, U.S. Geological Survey.)

3. Well near Clovis, California. (Photograph taken by Jennifer L. Shelton, U.S. Geological Survey.)

4. Well near Madera, California. (Photograph taken by Jennifer L. Shelton, U.S. Geological Survey.)

5. Near Chowchilla, California. (Photograph taken by Jennifer L. Shelton, U.S. Geological Survey.) 


\section{Groundwater-Quality Data for the Madera/ Chowchilla-Kings Shallow Aquifer Study Unit, 2013-14: Results from the California GAMA Program}

By Jennifer L. Shelton and Miranda S. Fram

Prepared in cooperation with the California State Water Resources Control Board

Data Series 1019 


\title{
U.S. Department of the Interior SALLY JEWELL, Secretary
}

\section{U.S. Geological Survey Suzette M. Kimball, Director}

\author{
U.S. Geological Survey, Reston, Virginia: 2016
}

For more information on the USGS - the Federal source for science about the Earth, its natural and living resources, natural hazards, and the environment—visit http://www.usgs.gov or call 1-888-ASK-USGS.

For an overview of USGS information products, including maps, imagery, and publications, visit http://www.usgs.gov/pubprod/.

Any use of trade, firm, or product names is for descriptive purposes only and does not imply endorsement by the U.S. Government.

Although this information product, for the most part, is in the public domain, it also may contain copyrighted materials as noted in the text. Permission to reproduce copyrighted items must be secured from the copyright owner.

Suggested citation:

Shelton, J.L., and Fram, M.S., 2017, Groundwater-quality data for the Madera/Chowchilla-Kings shallow aquifer study unit, 2013-14: Results from the California GAMA Program: U.S. Geological Survey Data Series 1019, 115 p., https://doi.org/10.3133/ds1019. 


\section{Contents}

Abstract

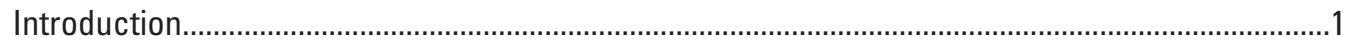

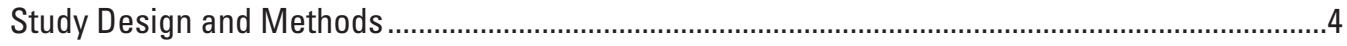

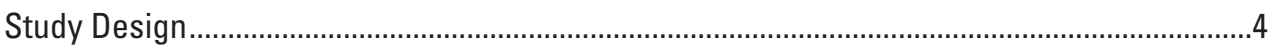

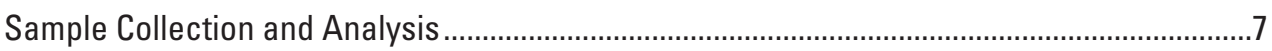

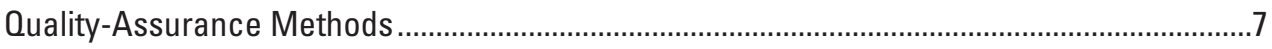

Water-Quality Results ...........................................................................................................

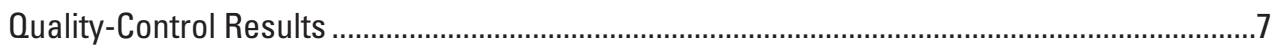

Comparative Benchmarks .......................................................................................................

Groundwater-Quality Data ....................................................................................................

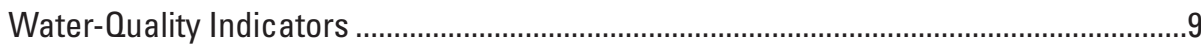

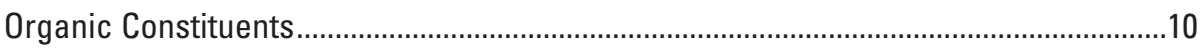

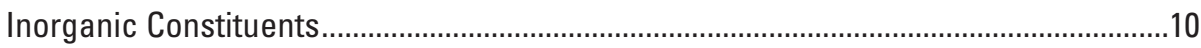

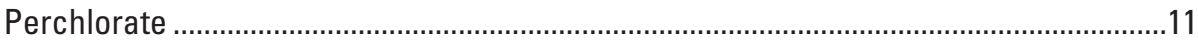

Radioactive Constituents............................................................................................11

Geochemical and Age-Dating Tracers........................................................................11

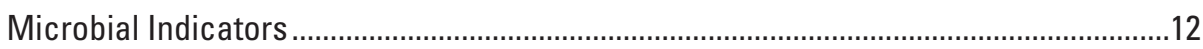

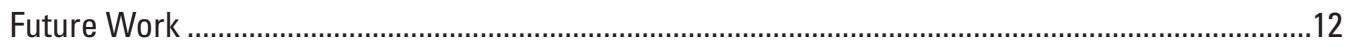

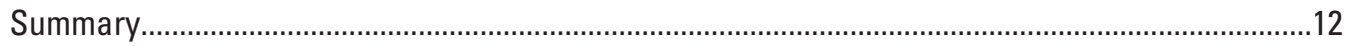

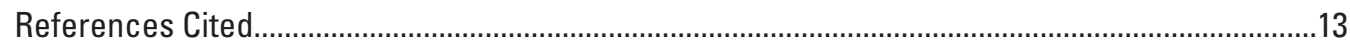

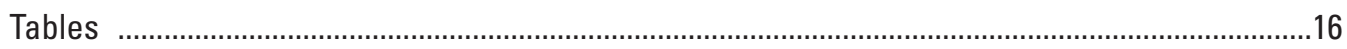

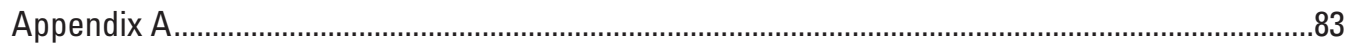

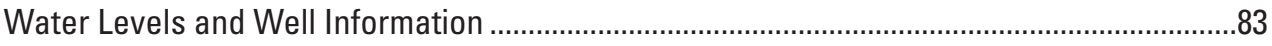

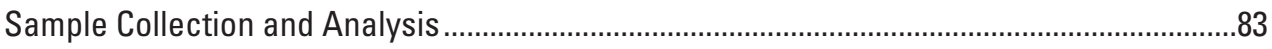

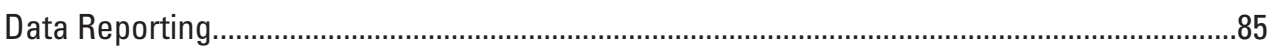

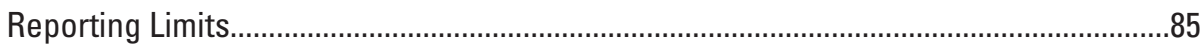

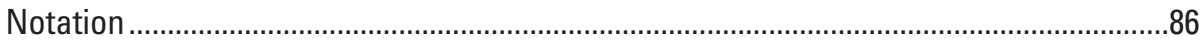

Constituents Determined by Multiple Analytical Methods ...............................................87

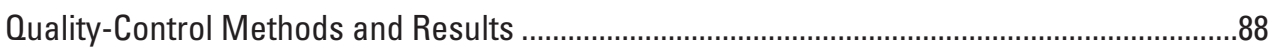

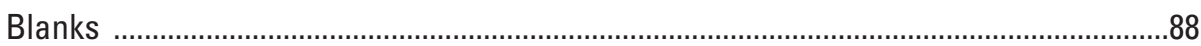

Blank Collection and Analysis ...........................................................................8

Study Reporting Levels (SRLs) Based on Results from Previous Study Units........89

SRLs Based on Detections in S3-MACK Blanks .....................................................89

Interim Study Reporting Levels Based on Laboratory Blank Data ........................90

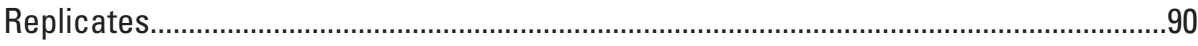

Assessment of Replicate Samples ......................................................................90

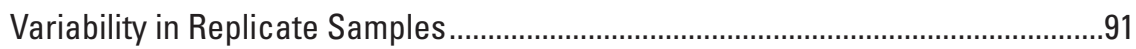

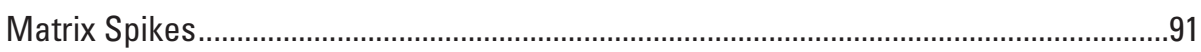

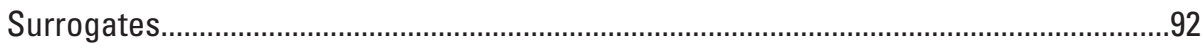

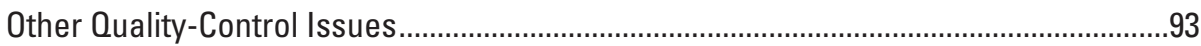

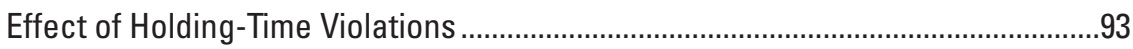

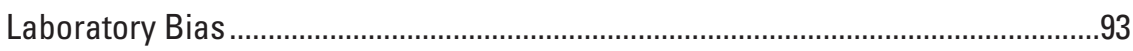

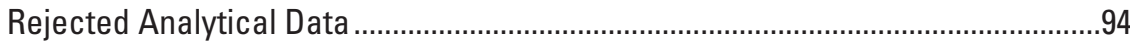

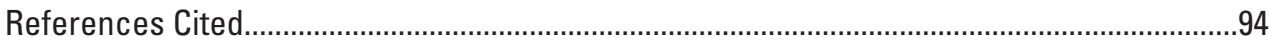




\section{Figures}

1. Map showing the hydrogeologic provinces of California and the location of the Madera/Chowchilla-Kings Shallow Aquifer study unit, Groundwater Ambient Monitoring and Assessment Priority Basin Project, California ...

2. Map showing the boundaries of the two study areas of the Madera/ChowchillaKings Shallow Aquifer study unit, as well as selected cities, topographic features, and hydrologic features...

3. Map showing the distribution of the Madera/Chowchilla and Kings study area cells, the sampled grid wells, and topographic features .

\section{Tables}

1. Identification, sampling, construction and water-level information for wells sampled in the Madera/Chowchilla-Kings Shallow Aquifer study unit, Groundwater Ambient Monitoring and Assessment Priority Basin Project, California, August 2013 to April 2014......

2. Classes of chemical constituents and field water-quality indicators measured for the Madera/Chowchilla-Kings Shallow Aquifer study unit, Groundwater Ambient Monitoring and Assessment Priority Basin Project, California, August 2013 to April 2014.......

3A. Volatile organic compounds, primary uses or sources, comparison benchmarks, and reporting information for the U.S. Geological Survey National Water Quality Laboratory Schedules 4436 and 4437

$3 B$. Pesticides and pesticide degradates, primary uses or sources, comparison benchmarks, and reporting information for the U.S. Geological Survey National Water Quality Laboratory Schedule 2437

3C. Inorganic constituents, comparative benchmarks, and reporting information ...............32

3D. Microbial indicators, comparative benchmarks, and reporting information ...................34

4. Water-quality indicators in groundwater samples collected for the Madera/ Chowchilla-Kings Shallow Aquifer study unit, Groundwater Ambient Monitoring and Assessment Priority Basin Project, California, August 2013 to April 2014

5. Volatile organic compounds detected in groundwater samples collected for the Madera/Chowchilla-Kings Shallow Aquifer study unit, Groundwater Ambient Monitoring and Assessment Priority Basin Project, California, August 2013 to April 2014.

6A. Pesticides detected in groundwater samples collected for the Madera/ Chowchilla-Kings Shallow Aquifer study unit, Groundwater Ambient Monitoring and Assessment Priority Basin Project, California, August 2013 to April 2014

6B. Pesticide degradates detected in samples collected for the Madera/ChowchillaKings Shallow Aquifer study unit, Groundwater Ambient Monitoring and Assessment Priority Basin Project, California, August 2013 to April 2014.

7. Results for analyses of trace elements in groundwater samples collected for the Madera/Chowchilla-Kings Shallow Aquifer study unit, Groundwater Ambient Monitoring and Assessment Priority Basin Project, California, August 2013 to April 2014.......

8. Results for analyses of nutrients in groundwater samples collected for the Madera/ Chowchilla-Kings Shallow Aquifer study unit, Groundwater Ambient Monitoring and Assessment Priority Basin Project, California, August 2013 to April 2014 


\section{Tables-Continued}

9. Results for analyses of major and minor ions, silica, and total dissolved solids in groundwater samples collected for the Madera/Chowchilla-Kings Shallow Aquifer study unit, Groundwater Ambient Monitoring and Assessment Priority Basin Project, California, August 2013 to April 2014

10. Results for analyses of perchlorate in groundwater samples collected for the Madera/Chowchilla-Kings Shallow Aquifer study unit, Groundwater Ambient Monitoring and Assessment Priority Basin Project, California, August 2013 to April 2014...

11. Results for analyses of radioactive constituents in groundwater samples collected for the Madera/Chowchilla-Kings Shallow Aquifer study unit, Groundwater Ambient Monitoring and Assessment Priority Basin Project, California, August 2013 to April 2014...

12. Results for analyses of isotopic tracers in groundwater samples collected for the Madera/Chowchilla-Kings Shallow Aquifer study unit, Groundwater Ambient Monitoring and Assessment Priority Basin Project, California, August 2013 to April 2014

13. Microbial indicators detected in groundwater samples collected for the Madera/ Chowchilla-Kings Shallow Aquifer study unit, Groundwater Ambient Monitoring and Assessment study, California, August 2013 to April 2014

A-1. Analytical methods used for the determination of organic and inorganic constituents in water by the U.S. Geological Survey National Water Quality Laboratory and contract laboratories..

A-2. Constituents detected in the blank water samples and the study reporting level analysis used for the Madera/Chowchilla-Kings Shallow Aquifer study unit, Groundwater Ambient Monitoring and Assessment Priority Basin Project, California, August 2013 to April 2014

A-3A. Quality-control summary for replicate pair analyses of organic and inorganic constituents in groundwater samples collected from the Madera/Chowchilla-Kings Shallow Aquifer study unit, Groundwater Ambient Monitoring and Assessment Priority Basin Project, California, August 2013 to April 2014

A-3B. Quality-control summary for replicate pair analyses of radioactive constituents in groundwater samples collected from the Madera/Chowchilla-Kings Shallow Aquifer study unit, Groundwater Ambient Monitoring and Assessment Priority Basin Project, California, August 2013 to April 2014

A-3C. Quality-control summary for replicate pair analyses of microbial indicators in groundwater samples collected from the Madera/Chowchilla-Kings Shallow Aquifer study unit, Groundwater Ambient Monitoring and Assessment Priority Basin Project, California, August 2013 to April 2014

A-4A. Quality-control summary for matrix-spike recoveries of volatile organic compounds in groundwater samples collected from the Madera/Chowchilla-Kings Shallow Aquifer study unit, Groundwater Ambient Monitoring and Assessment Priority Basin Project, California, August 2013 to April 2014

A-4B. Quality-control summary for matrix-spike recoveries of pesticides and pesticide degradates in samples collected for the Madera/Chowchilla-Kings Shallow Aquifer study unit, Groundwater Ambient Monitoring and Assessment Priority Basin Project, California, August 2013 to April 2014 


\section{Tables-Continued}

A-5. Quality-control summary for surrogate compound recoveries of volatile organic compounds and pesticides and pesticide degradates in groundwater samples collected from the Madera/Chowchilla-Kings Shallow Aquifer study unit, Groundwater Ambient Monitoring and Assessment Priority Basin Project, California, August 2013 to April 2014

\section{Conversion Factors, Datums, and Abbreviations and Acronyms}

\section{Conversion Factors}

Inch/foot/mile to International System of Units (SI)

\begin{tabular}{lll}
\hline \multicolumn{1}{c}{ Multiply } & \multicolumn{1}{c}{ By } & \multicolumn{1}{c}{ To obtain } \\
\hline inch (in.) & 2.54 & centimeter $(\mathrm{cm})$ \\
foot (ft) & 0.3048 & meter $(\mathrm{m})$ \\
mile (mi) & 1.609 & kilometer $(\mathrm{km})$ \\
square mile $\left(\mathrm{mi}^{2}\right)$ & 2.590 & square kilometer $\left(\mathrm{km}^{2}\right)$ \\
quart (qt) & 0.95 & liter $(\mathrm{L})$ \\
ounce, avoirdupois (oz) & 28.35 & gram $(\mathrm{g})$ \\
pound, avoirdupois (lb) & 0.4536 & kilogram $(\mathrm{kg})$ \\
\hline
\end{tabular}

SI to Inch/Pound

\begin{tabular}{lll}
\hline \multicolumn{1}{c}{ Multiply } & \multicolumn{1}{c}{ By } & \multicolumn{1}{c}{ To obtain } \\
\hline centimeter $(\mathrm{cm})$ & 0.3937 & inch (in.) \\
meter $(\mathrm{m})$ & 3.28 & foot (ft) \\
kilometer $(\mathrm{km})$ & 0.6214 & mile (mi) \\
square kilometer $\left(\mathrm{km}^{2}\right)$ & 0.3861 & square mile $\left(\mathrm{mi}^{2}\right)$ \\
liter $(\mathrm{L})$ & 1.0567 & quart (qt) \\
gram $(\mathrm{g})$ & 0.03527 & ounce, avoirdupois $(\mathrm{oz})$ \\
kilogram $(\mathrm{kg})$ & 2.205 & pound, avoirdupois $(\mathrm{lb})$ \\
\hline
\end{tabular}

Temperature in degrees Celsius $\left({ }^{\circ} \mathrm{C}\right)$ may be converted to degrees Fahrenheit $\left({ }^{\circ} \mathrm{F}\right)$ as follows:

${ }^{\circ} \mathrm{F}=\left(1.8 \mathrm{x}^{\circ} \mathrm{C}\right)+32$.

\section{Datum}

Vertical coordinate information is referenced to the North American Vertical Datum of 1988 (NAVD 88).

Horizontal coordinate information is referenced to the North American Datum of 1983 (NAD 83).

Altitude, as used in this report, refers to distance above the vertical datum and is reported as feet above mean sea level ( $\mathrm{ft}$ above $\mathrm{msl}$ ) and feet below mean sea level ( $\mathrm{ft}$ below $\mathrm{msl}$ ). 


\section{Supplemental Information}

Specific conductance is given in microsiemens per centimeter at 25 degrees Celsius $(\mu \mathrm{S} / \mathrm{cm}$ at $\left.25^{\circ} \mathrm{C}\right)$.

Concentrations of chemical constituents in water are given in either milligrams per liter $(\mathrm{mg} / \mathrm{L})$, micrograms per liter $(\mu \mathrm{g} / \mathrm{L})$, or nanograms per liter $(\mathrm{ng} / \mathrm{L})$.

Milligrams per liter is equivalent to parts per million (ppm), micrograms per liter is equivalent to parts per billion (ppb), and nanograms per liter is equivalent to parts per trillion (ppt).

Activities of radioactive constituents in water are given in picocuries per liter (pCi/L).

\section{Abbreviations}

$\begin{array}{ll}\text { AL-US } & \text { action level (EPA) } \\ \text { BBP } & \text { Blind Blank Program (USGS) } \\ \text { BOS } & \text { Branch of Quality Systems (USGS) } \\ \mathrm{CaCO}_{3} & \text { calcium carbonate } \\ \mathrm{CDWR}^{2-} & \text { California Department of Water Resources } \\ \mathrm{CO}_{3}^{2-} & \text { carbonate } \\ \text { CSU } & \text { combined standard uncertainty } \\ \text { DBCP } & \text { 1,2-dibromo-3-chloropropane } \\ \text { E. Coli } & \text { Escherichia coli } \\ \text { EDB } & \text { 1,2-dibromoethane } \\ \text { EPA } & \text { U.S. Environmental Protection Agency } \\ \text { GAMA } & \text { Groundwater Ambient Monitoring and Assessment Program } \\ \text { HAL-US } & \text { lifetime health-advisory level (EPA) } \\ \text { HBSL } & \text { health-based screening level (USGS) } \\ \text { HCO } & \text { bicarbonate } \\ \text { HHBP } & \text { human-health benchmark for pesticides (EPA) } \\ \text { IBSP } & \text { Inorganic Blind Sample Program (USGS) } \\ \text { IOR } & \text { interquartile range } \\ \text { IRL } & \text { interim reporting level } \\ \text { LLNL } & \text { Lawrence Livermore National Laboratory } \\ \text { LRL } & \text { laboratory reporting level } \\ \text { LSD } & \text { datum plane that is approximately at land surface at each well site } \\ \text { LT-MDL } & \text { long-term method detection level } \\ \text { MACK } & \text { Madera/Chowchilla-Kings Shallow Aquifer } \\ \text { MCL-CA } & \text { maximum contaminant level-State of California } \\ & \end{array}$


Abbreviations-Continued

\begin{tabular}{|c|c|}
\hline MCL-HI & maximum contaminant level-State of Hawaii \\
\hline MCL-US & maximum contaminant level (EPA) \\
\hline MDL & method detection limit \\
\hline MRL & minimum reporting level \\
\hline MTBE & Methyl tert-butyl ether \\
\hline MU & method uncertainty \\
\hline NAWQA & National Water Quality Assessment Program (USGS) \\
\hline NFM & National Field Manual (USGS) \\
\hline NL-CA & notification level (State of California) \\
\hline NWIS & National Water Information System (USGS) \\
\hline NWQL & National Water Quality Laboratory (USGS) \\
\hline PBP & Priority Basin Project \\
\hline PCFF & Personal Computer Field Form program designed for USGS sampling \\
\hline$Q A$ & quality assurance \\
\hline QC & quality control \\
\hline $\mathrm{RL}$ & reporting level \\
\hline RSD & relative standard deviation \\
\hline RSD5-US & EPA risk-specific dose at a risk factor of $10-5$ \\
\hline SC & specific conductance \\
\hline SD & standard deviation \\
\hline SMCL & secondary maximum contaminant level \\
\hline SMCL-CA & secondary maximum contaminant level (State of California) \\
\hline SMCL-US & secondary maximum contaminant level (EPA) \\
\hline SRL & $\begin{array}{l}\text { study reporting level (concentration cutoff for applying the less than or equal } \\
\text { to symbol in reporting results) }\end{array}$ \\
\hline $\mathrm{ssL}_{\mathrm{c}}$ & sample-specific critical level \\
\hline SWRCB & California State Water Resources Control Board \\
\hline TDS & total dissolved solids \\
\hline U.S. & United States \\
\hline USGS & U.S. Geological Survey \\
\hline VOC & volatile organic compound \\
\hline WCR & well-completion report (commonly called a driller's log) \\
\hline $1,2,3-\mathrm{TCP}$ & 1,2,3-trichloropropane \\
\hline
\end{tabular}




\title{
Groundwater-Quality Data for the Madera/Chowchilla- Kings Shallow Aquifer Study Unit, 2013-14: Results from the California GAMA Program
}

\author{
By Jennifer L. Shelton and Miranda S. Fram
}

\section{Abstract}

Groundwater quality in the 2,390-square-mile Madera/ Chowchilla-Kings Shallow Aquifer study unit was investigated by the U.S. Geological Survey from August 2013 to April 2014 as part of the California State Water Resources Control Board Groundwater Ambient Monitoring and Assessment Program's Priority Basin Project. The study was designed to provide a statistically unbiased, spatially distributed assessment of untreated groundwater quality in the shallow aquifer systems of the Madera, Chowchilla, and Kings subbasins of the San Joaquin Valley groundwater basin. The shallow aquifer system corresponds to the part of the aquifer system generally used by domestic wells and is shallower than the part of the aquifer system generally used by public-supply wells. This report presents the data collected for the study and a brief preliminary description of the results.

Groundwater samples were collected from 77 wells and were analyzed for organic constituents, inorganic constituents, selected isotopic and age-dating tracers, and microbial indicators. Most of the wells sampled for this study were private domestic wells. Unlike groundwater from publicsupply wells, the groundwater from private domestic wells is not regulated for quality in California and is rarely analyzed for water-quality constituents. To provide context for the sampling results, however, concentrations of constituents measured in the untreated groundwater were compared with regulatory and non-regulatory benchmarks established for drinking-water quality by the U.S. Environmental Protection Agency, the State of California, and the U.S. Geological Survey.

Of the 319 organic constituents assessed in this study (90 volatile organic compounds and 229 pesticides and pesticide degradates), 17 volatile organic compounds and 23 pesticides and pesticide degradates were detected in groundwater samples; concentrations of all but 2 were less than the respective benchmarks. The fumigants 1,2-dibromo3-chloropropane (DBCP) and 1,2-dibromoethane (EDB) were detected at concentrations above their respective regulatory benchmarks in samples from a total of four wells.
Most detections of inorganic constituents were at concentrations or activities less than the respective benchmark levels. Five inorganic constituents were detected in groundwater samples from one or more wells at concentrations or activities greater than their respective regulatory, healthbased benchmarks: arsenic, uranium, nitrate, adjusted gross alpha particle activity, and gross beta particle activity. Four inorganic constituents were detected in samples from one or more wells at concentrations or activities greater than their respective non-regulatory, health-based benchmarks: manganese, molybdenum, vanadium, and radon-222. Three inorganic constituents were detected in groundwater samples from one or more wells at concentrations greater than their respective non-regulatory, aesthetic-based benchmarks: iron, sulfate, and total dissolved solids.

Microbial indicators (Escherichia coli, total coliform, and enterococci) were analyzed for presence or absence. The presence of Escherichia coli (E. coli) was not detected; the presence of total coliform was detected in samples from 10 of the 72 grid wells for which it was analyzed, and the presence of enterococci was detected in samples from 5 of the 73 grid wells analyzed.

\section{Introduction}

About one-half of the water used for public and domestic drinking-water supply in California is groundwater (Kenny and others, 2009). To assess the quality of ambient groundwater in aquifers used for drinking-water supply and to establish a baseline groundwater-quality monitoring program, the California State Water Resources Control Board (SWRCB), in cooperation with the U.S. Geological Survey (USGS) and Lawrence Livermore National Laboratory (LLNL), implemented the Groundwater Ambient Monitoring and Assessment (GAMA) Program (http://www.waterboards. ca.gov/gama/). The SWRCB initiated the GAMA Program in 2000 in response to a legislative mandate (State of California, 2001a, 2001b). The main goals of the GAMA Program are to improve groundwater monitoring and to increase the amount and availability of groundwater-quality data to the public. 
The GAMA Program currently consists of four projects: (1) the GAMA Priority Basin Project (PBP), led by the USGS (http://ca.water.usgs.gov/gama/); (2) the GAMA Domestic Well Project, led by the SWRCB; (3) the GAMA Special Studies Project, led by LLNL; and (4) the GeoTracker GAMA on-line groundwater information system, led by the SWRCB (http://geotracker.waterboards.ca.gov/gama/). The private domestic wells were sampled for the SWRCB's GAMA Domestic Well Project on a voluntary basis in six counties between 2002 and 2011. From 2004 through 2012, the GAMA Priority Basin Project assessed the water quality of groundwater resources used for public drinking-water supplies. The groundwater resources used for public drinkingwater supplies typically are deeper than the groundwater resources used for domestic drinking-water supplies. In 2012, the GAMA Priority Basin Project began water-quality assessments of shallow aquifers, the groundwater resources typically used for private domestic and small-system drinkingwater supplies.

The GAMA-PBP was initiated in response to the Groundwater Quality Monitoring Act of 2001 to assess and monitor the quality of groundwater in California (State of California, 2001b). For the first phase of the GAMA-PBP, the USGS, in collaboration with the SWRCB, developed a monitoring plan to assess groundwater resources used for public drinking-water supply through direct sampling of groundwater and other statistically reliable sampling approaches (Belitz and others, 2003; California State Water Resources Control Board, 2003). Groundwater basins and areas outside of basins were prioritized for sampling primarily on the basis of the distribution of wells listed in the State of California's database of public-supply wells. Responsibility for regulation of public drinking-water sources was transferred from the Drinking Water Program of the California Department of Public Health to the Division of Drinking Water of the SWRCB on July 1, 2014. The 35 study units sampled in this first phase of the GAMA-PBP (2004-12) covered nearly all of the groundwater resources used for public-supply statewide (Belitz and others, 2015).

For the second phase of the GAMA-PBP, a different method of prioritization was required because there is no statewide database of private wells to use to prioritize areas for sampling. To prioritize shallow aquifers, California was divided into 938 groundwater units corresponding to the 463 alluvial groundwater basins defined by the California Department of Water Resources (CDWR; California Department of Water Resources, 2003) and 453 areas outside of basins (Johnson and Belitz, 2014). The distribution of households relying on domestic wells was estimated from U.S. Census data (U.S. Census Bureau, 1990), and water-use and well-location information were compiled from wellcompletion reports (WCRs) submitted to the CDWR (Johnson and Belitz, 2015). Groundwater units were prioritized for sampling on the basis of the number and density of households relying on domestic wells. Groundwater units were grouped into study units designed to facilitate comparison of groundwater quality between the shallow aquifer systems assessed in this second phase of the GAMA-PBP and the deeper aquifer systems assessed in the first phase. The depth of the shallow aquifer system is defined as shallower than the primary aquifer system, which is the depth interval tapped by public-supply wells. In many groundwater basins, domestic and small-system wells typically are shallower than publicsupply wells; thus, the shallow aquifer system typically corresponds to the depth interval tapped by domestic and small-system wells (for example, Burow and others, 2008; Burton and others, 2012). This depth difference between domestic and public-supply wells can be less distinct in some areas.

The Madera/Chowchilla-Kings Shallow Aquifer (MACK) study unit in the Central Valley hydrogeologic province was the third study unit sampled during the second phase of the GAMA-PBP (fig. 1).

Three types of water-quality assessments are completed with the data collected in each study unit: (1) status, an assessment of the current quality of the groundwater resource; (2) understanding, the identification of the natural and human factors affecting groundwater quality; and (3) trends, the detection of changes in groundwater quality (Kulongoski and Belitz, 2004). These three objectives were modeled after those of the USGS National Water Quality Assessment (NAWQA) Program (Hirsch and others, 1988).

This USGS data-series report is similar to other USGS data-series reports about the GAMA-PBP study units sampled to date and is the first in a series of reports (for example, dataseries report, scientific investigations report, and fact sheet) presenting the data collected and assessments of water quality in the MACK study unit. The water-quality data presented in this report also are available from the USGS National Water Information System (NWIS) Web (http://waterdata.usgs.gov/ ca/nwis/) and GeoTracker GAMA (http://www.waterboards. ca.gov/gama/geotracker_gama.shtml). Data-series reports and additional reports addressing the status, understanding, and trends aspects of the water-quality assessments of each study unit are available at the project website http://ca.water.usgs. gov/gama/includes/GAMA_publications.html.

The purposes of this report are to (1) describe the study design and methods used to collect the data, (2) present the analytical results for groundwater samples, and (3) present the evaluation of results from quality-control samples. Samples were analyzed for field water-quality indicators, organic and inorganic constituents, isotopic tracers, and microbial indicators. To provide context, water-quality data were compared to drinking-water-quality benchmarks established by the U.S. Environmental Protection Agency (EPA), the SWRCB-Division of Drinking Water, and the USGS. Discussion of the factors that influence the distribution of the constituents detected in groundwater samples is the subject of subsequent publications. 


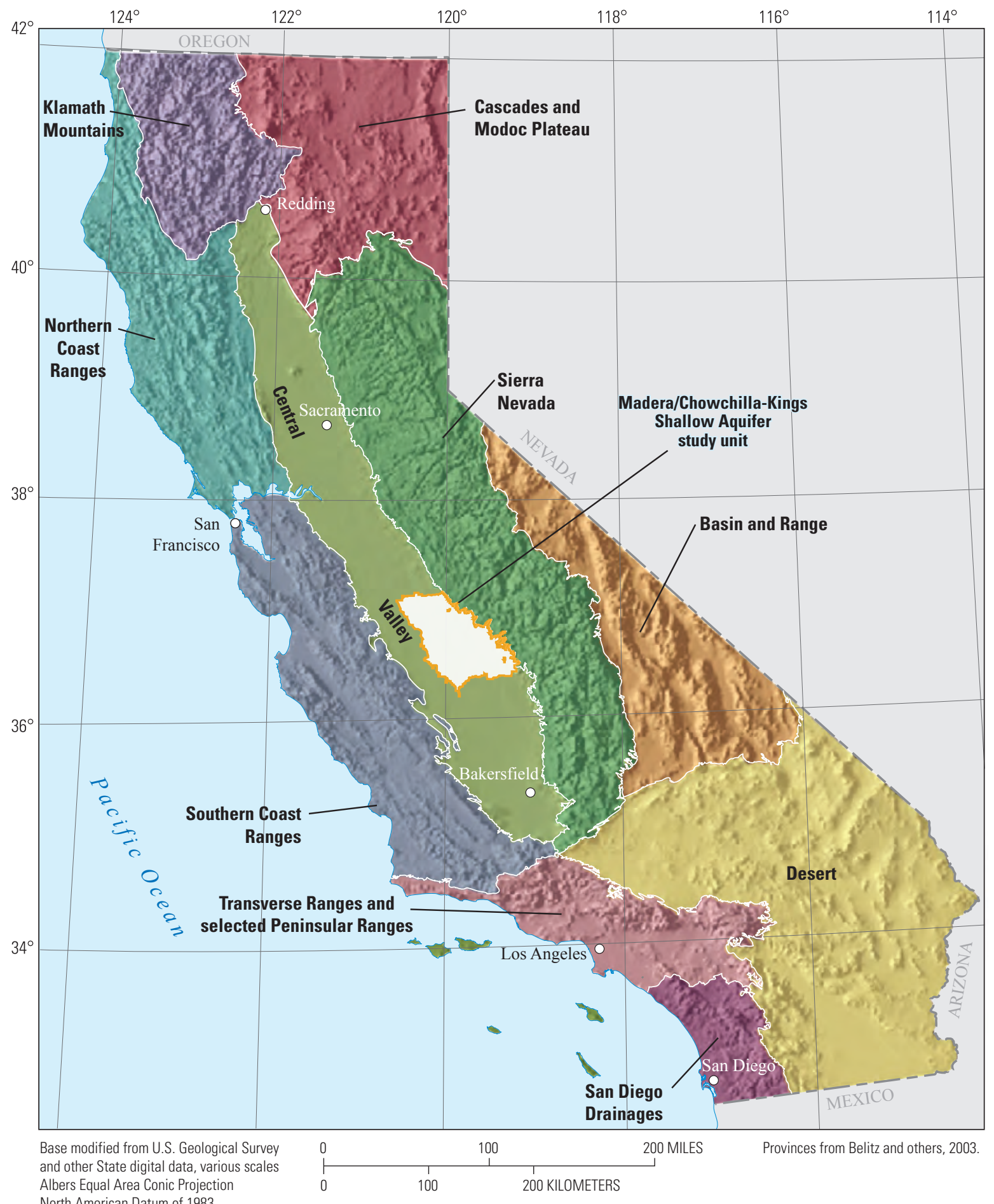

Figure 1. Hydrogeologic provinces of California and the location of the Madera/Chowchilla-Kings Shallow Aquifer study unit, Groundwater Ambient Monitoring and Assessment (GAMA) Priority Basin Project, California. 


\section{Study Design and Methods}

The methods used for the GAMA-PBP were selected to achieve the following objectives: (1) design a sampling plan for suitable statistical representation of the MACK study unit, (2) collect samples in a consistent manner, (3) analyze samples by using proven and reliable laboratory methods, (4) assure the quality of the groundwater data, and (5) maintain data securely and with relevant documentation. The appendix contains detailed descriptions of the sample-collection protocols, analytical methods, the quality-assurance (QA) methods, and the results of analyses of quality-control (QC) samples.

\section{Study Design}

The MACK study unit covers approximately 2,390 square miles $\left(\mathrm{mi}^{2}\right)$ in the Central Valley hydrogeologic province described by Belitz and others (2003; fig. 1). The study unit is divided into two study areas: the Madera/ Chowchilla study area and the Kings study area (fig. 2). These study areas correspond to the same study areas used for the GAMA-PBP assessment of public-supply aquifers: the Madera-Chowchilla study unit (Shelton and others, 2009, 2013) and the Kings study area of the Southeast San Joaquin Valley study unit (Burton and Belitz, 2008; Burton and others, 2012). These references give detailed descriptions of the hydrogeologic settings of the study areas in the MACK study unit; only a brief summary is provided here in the paragraphs that follow.

The Madera/Chowchilla study area was defined by the extent of the Madera and Chowchilla groundwater subbasins of the San Joaquin Valley groundwater basin (California Department of Water Resources, 2003, 2008a, and 2008b). The study area covers an area of approximately $860 \mathrm{mi}^{2}$, primarily in Madera County, but with a small part in Merced County north of the Chowchilla River and in Fresno County south of the San Joaquin River. The primary aquifer system in the San Joaquin Valley consists of Quaternary-age unconsolidated continental deposits. The deposits are composed of alluvial fan and fluvial gravel, sand, silt, and clay sediments interbedded with lesser amounts of lacustrine deposits. The sediments were derived from the weathering of the granitic rocks of the Sierra Nevada to the east. The sediments generally are coarsest in the eastern part of the valley along the foothills and become finer westward toward the San Joaquin River, the axial trough of the San Joaquin Valley (Gronberg and others, 1998). The continental deposits bearing freshwater increase in thickness from north to south in the San Joaquin Valley and are up to 3,000 feet (ft) thick in the Madera/Chowchilla study area (Bertoldi and others, 1991). The Corcoran Clay, the thickest of the lacustrine deposits, is present in the western half of the Madera/Chowchilla study area.
The Kings study area was defined by the boundaries of the CDWR-defined Kings groundwater subbasin of the San Joaquin Valley groundwater basin and includes $1,530 \mathrm{mi}^{2}$ of Fresno County and the northern part of Kings and Tulare Counties (California Department of Water Resources, 2003 and 2006). The San Joaquin River borders the subbasin to the north, and the Kings River flows through the eastern portion of the study area, eventually bordering it to the south. The aquifer system consists of marine and continental deposits of Tertiary to Quaternary age overlain by younger alluvial deposits of Quaternary age (Davis and others, 1959; Faunt, 2009). The eastern two-thirds of the study area consist of the Quaternary alluvial fans of the Kings River, with compound alluvial fans of intermittent streams to the north and south (Burow and others, 1997). These deposits generally are highly permeable. The western one-third of the study area, near the center of the San Joaquin valley, includes less permeable deposits in the Basin and Range geomorphic province (Davis and others, 1959; Faunt, 2009). Vertical flow is increasingly restricted by discontinuous silt and clay layers toward the valley center. The Corcoran Clay member of the Tulare Formation, ranging in depth from about 250 to $550 \mathrm{ft}$, is the most extensive clay layer and forms a regional confining unit (California Department of Water Resources, 2006).

To produce a statistically unbiased, spatially distributed assessment of the quality of groundwater resources in the shallow aquifer system, wells were selected by using a randomized, grid-based method. The study unit was divided into equal-area grid cells (Scott, 1990), and one well was randomly selected for sampling in each cell. These wells are referred to as "grid wells." Each grid cell was $31 \mathrm{mi}^{2}$ and approximately rectangular, although the shapes of the cells may vary or geographic features may force a grid cell to be divided into multiple pieces to obtain the designated coverage area for each cell. On the basis of the different sizes of the study areas, the Madera/Chowchilla study area was divided into 28 grid cells, and the Kings study area was divided into 49 grid cells.

The study objective was to sample one shallow well in each grid cell (fig. 3). Shallow wells were targeted by using WCR information obtained from the CDWR. The WCRs of shallow wells (generally private domestic and small-system wells) that contained location information were compiled for each cell. To ensure that wells were randomly selected, a random latitude/longitude point was defined in each cell, and wells closest to the random point were given highest priority. Locations were visited in descending order of priority until voluntary permission to sample an appropriate well was obtained. Basic sampling criteria (for example, a sampling point prior to any treatment application, such as chlorination, or the capability to pump for an extended time) were considered prior to site selection for sampling. 


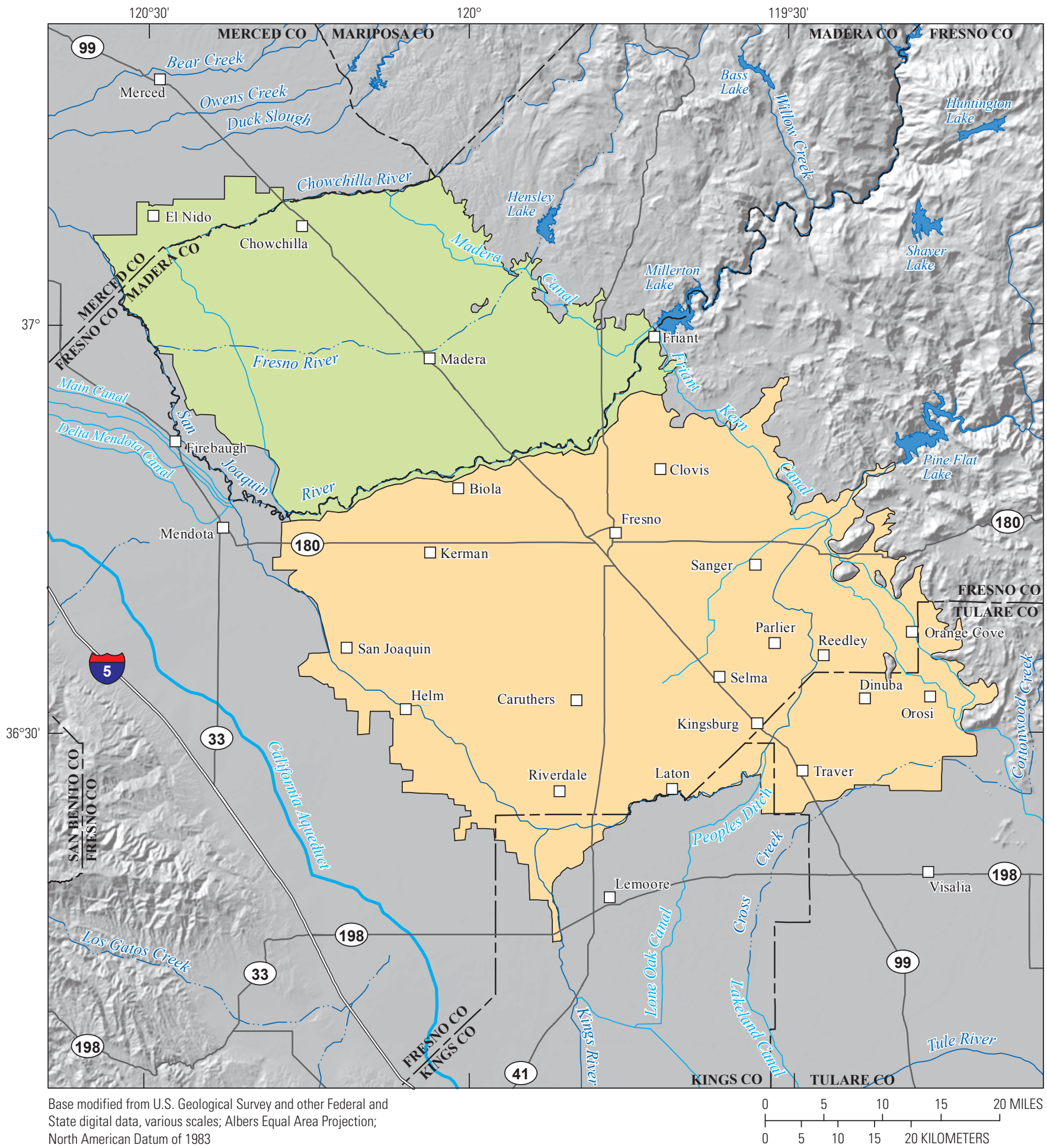

EXPLANATION

Madera/Chowchilla-Kings (MACK) shallow aquifer study unit

Kings study area

Madera/Chowchilla study area

Figure 2. Boundaries of the two study areas of the Madera/Chowchilla-Kings Shallow Aquifer study unit, as well as selected cities, topographic features, and hydrologic features. 


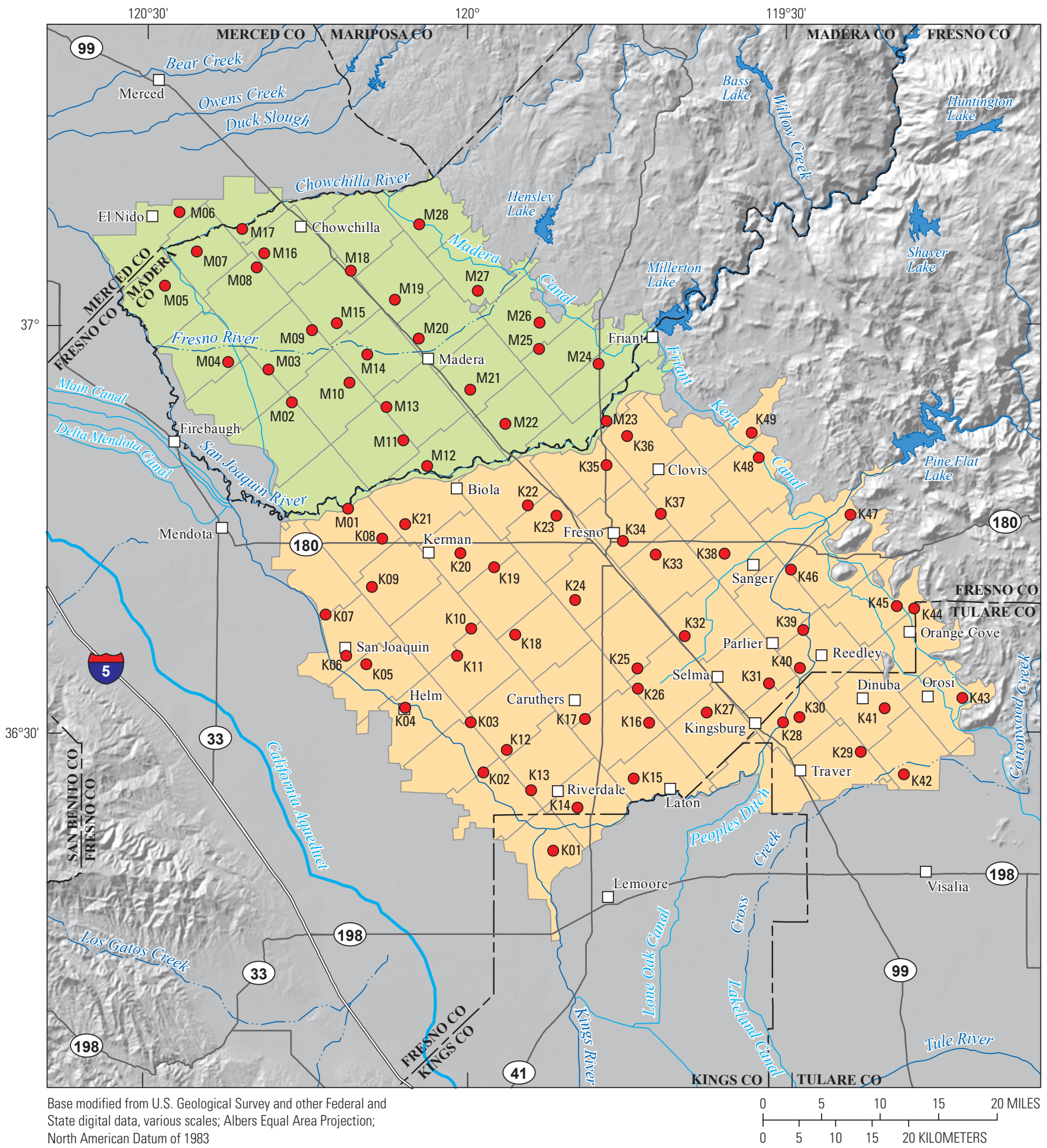

EXPLANATION

Madera/Chowchilla-Kings (MACK) shallow aquifer study unit

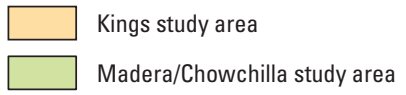

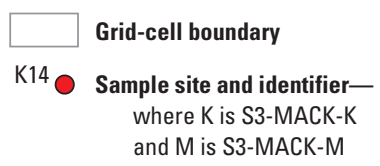

Figure 3. Distribution of the Madera/Chowchilla and Kings study area cells, the sampled grid wells, and topographic features. 
The 77 grid wells sampled in the MACK study unit were named by using the prefix "S3-MACK-M" for wells in the Madera/Chowchilla study area or "S3-MACK-K" for wells in the Kings study area (fig. 3). The "S3" portion of the prefix indicates the well is part of the third GAMA Shallow Aquifer Study well network. A sequence number defined by the number of the grid cell in which the samples were collected in each study area was then added, creating a unique alphanumeric GAMA identification number.

The GAMA identification number for each well, along with the date sampled, well type, well altitude, available wellconstruction information, and depth to water level are shown in table 1. Groundwater samples were collected during the period from August 2013 to April 2014. Well types in table 1 are identified as production or monitoring. Grid wells included 6 monitoring wells and 71 production wells, of which 68 were domestic wells, 2 were irrigation wells, and 1 was a small system public-supply well.

\section{Sample Collection and Analysis}

The methods used for sample collection and analysis are described in the appendix. Samples were collected in accordance with protocols modified from the USGS NAWQA program (Koterba and others, 1995) and the USGS National Field Manual (NFM; U.S. Geological Survey, variously dated). These sampling protocols were followed so that representative samples of groundwater were collected at each well and to ensure that the samples were collected and handled in a manner that minimizes the potential for contamination.

All 77 grid wells in the MACK study unit were sampled for a standard set of constituents (table 2). Tables $3 A-D$ list the compounds analyzed in each constituent class. Groundwater samples were analyzed for volatile organic compounds (VOCs; table $3 A$ ); pesticides and pesticide degradates (table $3 B$ ); inorganic constituents, including trace elements, major ions, nutrients, perchlorate, radioactive constituents, and geochemical and age-dating tracers (table $3 C$ ); and microbial indicators (table $3 D$ ).

\section{Quality-Assurance Methods}

The QA procedures and QC results are described in detail in the appendix. The QA procedures used for this study followed the protocols used by the NAWQA Program (Koterba and others, 1995) and are described in the USGS National Field Manual (U.S. Geological Survey, variously dated). The QC samples collected in the MACK study unit were blanks, replicates, and matrix and surrogate spikes. The QC samples were collected to evaluate potential contamination, as well as bias and variability in the data, that could have resulted from sample collection, processing, storage, transportation, or laboratory analysis.

\section{Water-Quality Results}

\section{Quality-Control Results}

Results of QC analyses were used to evaluate the quality of the data from the groundwater samples. On the basis of detections in field blanks collected for this and previous GAMA-PBP study units, "study reporting levels" (SRLs) for two VOCs and seven trace elements were used (see additional discussion in the appendix sections "Study Reporting Levels (SRLs) Based on Results for Previous Study Units" and "SRLs Based on Detections in S3-MACK Blanks"). Detections of these constituents at concentrations less than their SRLs either were reported as non-detections (VOCs) or flagged with a "less than or equal to symbol" (trace elements) in this report. On the basis of detections in the National Water Quality Laboratory prep blanks, an "interim study reporting level" (iSRL) was defined for three pesticides and one pesticide degradate (see appendix section "Interim Study Reporting Levels Based on Laboratory Blank Data"). Pesticides and pesticide degradates reported with concentrations less than iSRLs are not reported and are not counted as detections for the purposes of calculating detection frequencies.

Results from the replicates confirm that the procedures used to collect and analyze the samples were consistent. Variability for nearly 100 percent of the replicate pairs of constituents detected in samples was within the acceptable limits (see appendix tables A-3A-C). The criteria for acceptable replication are described in the appendix section "Replicates." Median matrix-spike recoveries for 1 of the 90 VOCs analyzed (butane) were less than the acceptable limit of 70 percent, and none were greater than the acceptable limit of 130 percent (appendix table A-4A). The constituents that had low recoveries possibly were not detected in some samples where they were present at concentrations near the laboratory reporting levels (LRLs). High recoveries of constituents indicate that reported values could be greater than what is in the sample. The QC results for spikes and surrogates are presented in the appendix sections titled "Matrix Spikes" and "Surrogates."

\section{Comparative Benchmarks}

The sample collection protocols used in this study were designed to obtain representative samples of groundwater. The quality of groundwater can differ from the quality of drinking water because water chemistry can change as a result of contact with plumbing systems or the atmosphere or because of treatment, disinfection, or blending with water from other sources. Water quality in domestic wells is not regulated in California; however, to provide context for the water-quality data presented in this report, results were compared to regulatory and non-regulatory benchmarks established for drinking-water quality. A constituent may have values for more than one type of benchmark, and the different benchmarks were prioritized to develop a consistent set of comparisons. For the second phase of the GAMA Priority Basin Project, benchmarks were selected in a specific order of priority. 
The highest priority benchmarks are regulatory benchmarks established by the EPA and the State of California that apply to public water systems and are designed to protect public health by limiting the levels of contaminants in drinking water (U.S. Environmental Protection Agency, 2012; California State Water Resources Control Board, 2013). These benchmarks include maximum contaminant levels (MCLs), action levels (ALs), and treatment technique requirements (TTs). The MCLs established by the EPA are the minimum standards with which states are required to comply, but individual states may choose to set standards that are more stringent. The State of California has established MCLs for constituents not regulated by the EPA and has lowered the benchmark concentrations for a number of constituents that have MCLs established by the EPA. In this report, a benchmark set by the EPA and adopted by the State is labeled "MCL-US," and one set by the State that is more stringent than the MCL-US is labeled "MCL-CA." Well owners were notified when constituents were detected at concentrations greater than the MCL-US or MCL-CA benchmark in samples collected for the GAMA-PBP, but these detections did not constitute violations of state regulations. For lead and copper, detections in public-water systems at levels greater than the benchmarks trigger requirements for mandatory water treatment to reduce the corrosiveness of water to distribution pipes. The ALs for lead and copper established by the EPA and the State were the same; thus, these benchmarks are labeled "AL-US" in this report. The benchmarks for microbial indicators in groundwater are established by the EPA and include an MCL-US benchmark for Escherichia coli (E.coli) and treatment technique thresholds (TT-US) for total coliform and for other potential fecal indicators (table $3 D$ ). Detections in public-water systems at levels greater than the benchmarks may trigger requirements for re-testing to verify results, assessments of the sanitary integrity of the groundwater source and distribution system, public notification, and additional disinfection (U.S. Environmental Protection Agency, 2016). Detections of microbial indicators do not mean pathogenic bacteria are present; rather, such detections indicate the system could be vulnerable to pathogen contamination from fecal sources. A total of 62 constituents analyzed for in this study had MCL-US, MCL-CA, AL-US, or TT-US benchmarks.

The second priority benchmarks are non-regulatory, health-based benchmarks for drinking-water quality: healthbased screening levels (HBSLs) that were developed by the USGS in collaboration with the EPA and others (Toccalino and others, 2012) and human-health benchmarks for pesticides (HHBPs) established by the EPA (U.S. Environmental Protection Agency, 2013). The HHBPs are levels of certain pesticides in water at or below which adverse health effects are not anticipated from 1-day or lifetime exposures (U.S. Environmental Protection Agency, 2013). The HHBPs and HBSLs are established by using the same methodologies implemented by the EPA when establishing drinking-water guidelines and are based on the most recent human-health toxicity information available (Toccalino and others, 2012; U.S. Environmental Protection Agency, 2013). The HHBPs and HBSLs are not established for constituents that have MCL-US benchmarks. The HBSLs have been established for some constituents that also have EPA non-regulatory health-based benchmarks and for a great number of organic constituents for which the EPA has not yet established benchmarks. For carcinogenic constituents, the HBSL range represents the constituent concentration in drinking water that represents an excess estimated lifetime cancer risk of one chance in one million to one chance in ten thousand. For noncarcinogens, the HBSL concentration is the maximum concentration in drinking water to which a lifetime of exposure is not expected to cause any adverse effect. The HBSLs follow the EPA assumptions about lifetime ingestion and are calculated assuming consumption of 2 liters (L; 2.1 quarts) of water per day over a 70-year lifetime by a 70-kilogram (kg; 154-pound) adult and that 20 percent of a person's exposure comes from drinking water. A total of 124 constituents analyzed for in this study have HBSL or HHBP benchmarks.

The third priority benchmarks are other non-regulatory, health-based benchmarks established by the EPA or the State of California (U.S. Environmental Protection Agency, 2012; California State Water Resources Control Board, 2010). These benchmarks are, in order of priority, the EPA lifetime health advisory level (HAL-US), the EPA risk-specific dose (RSD5-US), and the State of California notification level (NL-CA). A total of nine constituents analyzed for in this study had HAL-US, RSD5-US, or NL-CA benchmarks and did not also have HBSL benchmarks. The HAL-US is the maximum concentration of a constituent in drinking water that is not expected to cause any adverse health effects from a lifetime of exposure; it is calculated by assuming consumption of $2 \mathrm{~L}$ (2.1 quarts) of water per day during a 70 -year lifetime by a $70-\mathrm{kg}$ (154-pound) adult and that 20 percent of a person's exposure comes from drinking water (U.S. Environmental Protection Agency, 2012). Because the methods used to calculate HAL-US and HBSL benchmarks are the same, if constituents have a HAL-US and an HBSL, the two generally are the same value. The RSD5-US is the concentration of a constituent in drinking water corresponding to an excess estimated lifetime cancer risk of 1 in 100,000. The term "RSD5" is an acronym for risk-specific dose at the $10^{-5}$ risk level $\left(10^{-5}\right.$ equals $\left.1 / 100,000\right)$. An RSD5 is calculated by dividing the $10^{-4}$ cancer-risk concentration by 10 (U.S. Environmental Protection Agency, 2012). The NL-CA is a health-based standard established by the State for some of the constituents in drinking water that lack MCLs (California State Water Resources Control Board, 2010). If a constituent is detected in drinking water from a public water system at concentrations greater than its NL-CA, California State law requires timely notification of local governing bodies and recommends consumer notification. 
The fourth priority benchmarks are secondary maximum contaminant levels (SMCLs). The SMCLs are non-regulatory standards applied to constituents that affect the aesthetic qualities of drinking water, such as taste, odor, and color, or the technical qualities of drinking water, such as scaling and staining (California State Water Resources Control Board, 2010; U.S. Environmental Protection Agency, 2012). The EPA and the State both define SMCLs, but unlike MCLs, the SMCLs established by the State are not required to be at least as stringent as those established by the EPA. For chloride, sulfate, specific conductance, and total dissolved solids, the State of California defines a "recommended" and an "upper" SMCL-CA; detections of these constituents in groundwater samples were compared with the upper level. The SMCL-US levels for these constituents correspond to their recommended SMCL-CAs. The SMCLs established by the State are used in this report (SMCL-CA) for all constituents that have SMCL-CA values. The SMCL-US was used for $\mathrm{pH}$ because no SMCL-CA has been defined. A total of six constituents in this study had SMCL benchmarks.

Using this hierarchy to select the comparative benchmark for a constituent with more than one type of established benchmark does not necessarily result in selection of the benchmark with the lowest concentration. For example, the HBSL for boron is 6,000 micrograms per liter $(\mu \mathrm{g} / \mathrm{L})$, and the NL-CA is $1,000 \mu \mathrm{g} / \mathrm{L}$, but the comparative benchmark of highest priority selected by this hierarchy is the HBSL. The comparative benchmarks used in this report are listed in tables $3 A-D$ for all constituents and used in tables 4-13 for constituents detected in groundwater samples from the MACK study unit. Established benchmarks were not available for all constituents analyzed for this study. Detections of constituents at concentrations greater than the selected comparative benchmarks are marked with an asterisk in tables 4-13.

\section{Groundwater-Quality Data}

Results from analyses of untreated groundwater samples from the MACK study unit are presented in tables 4-13.

For organic constituents, the results tables include only those constituents that were detected and only those samples that had detections. Of the 319 organic constituents ( 90 VOCs and 229 pesticides) analyzed, 17 VOCs and 23 pesticides or pesticide degradates were detected in samples collected for the MACK study unit. For the organic constituents, the following summary statistics are presented in tables 5 and 6 for all of the grid wells: the number of grid wells at which each constituent was detected, the frequency at which it was detected (in relation to the number of grid wells in the study unit), and the total number of constituents detected at each well.

Water-quality indicators measured in the field and at the National Water Quality Laboratory (NWQL) are included in table 4 . The results of groundwater analyses, organized by constituent class, are presented in tables 5-13:
- Organic constituents.

- Volatile organic compounds (table 5).

- Pesticides and pesticide degradates (table $6 A, B$ ).

- Inorganic constituents.

- Trace elements (table 7).

- Nutrients (table 8).

- Major and minor ions, silica, and total dissolved solids (table 9).

- Perchlorate (table 10).

- Radioactive constituents (table 11).

- Isotopic tracers (table 12).

- Microbial indicators (table 13).

The tables of results for organic constituents and microbial indicators list only those samples in which one or more VOC (table 5), pesticide or pesticide degradate (table $6 \mathrm{~A}$, $B$ ), or microbial indicator (table 13) was detected and list only those constituents that were detected in one or more samples. The tables of results for inorganic constituents list all samples and all constituents.

\section{Water-Quality Indicators}

Field measurements of dissolved oxygen (DO) and water temperature, and field and laboratory measurements of $\mathrm{pH}$, specific conductance (SC), and alkalinity are presented in table 4. Bicarbonate and carbonate concentrations were calculated from the $\mathrm{pH}$ and alkalinity results. Alkalinity and DO are used as indicators of natural processes that affect water chemistry. The $\mathrm{pH}$ value indicates the acidity or alkalinity of the water. The SC is a measure of electrical conductivity of the water and is proportional to amount of total dissolved solids (TDS) in the water. The EPA and the State of California established non-regulatory benchmarks (SMCL-USs or SMCL-CAs) for $\mathrm{pH}$ and SC that are based on aesthetic properties rather than on human-health concerns.

Field $\mathrm{pH}$ values were outside of the SMCL-US range for 4 percent of the MACK study-unit grid wells: one well sample had a field $\mathrm{pH}$ value less than 6.5 standard units, and two well samples had field $\mathrm{pH}$ values greater than 8.5 standard units (table 4). Low pH in water can contribute to corrosion of pipes, and high $\mathrm{pH}$ in water can contribute to scaling. Laboratory $\mathrm{pH}$ values can differ from field $\mathrm{pH}$ values because the $\mathrm{pH}$ of groundwater can change when removed from the ambient environment and exposed to the atmosphere. Specificconductance values were greater than the upper SMCL-CA of 1,600 microsiemens per centimeter at 25 degrees Celsius in samples from 5 of the 77 grid wells (table 4). 


\section{Organic Constituents}

Volatile organic compounds (VOCs) are present in paints, solvents, fuels, fuel additives, refrigerants, fumigants, and disinfected water and are characterized by their tendency to evaporate. Generally, VOCs persist longer in groundwater than in surface water because groundwater generally is isolated from the atmosphere.

Of the 90 VOCs analyzed for the MACK study unit, 17 were detected in groundwater samples, and all but 2 were detected at concentrations that were less than health-based benchmarks (table 5). Two fumigants were detected at concentrations greater than health-based benchmarks in samples from a total of four grid wells: 1,2-dibromo-3-chloropropane (DBCP) from three grid wells and 1,2-dibromoethane (1,2-EDB) from one grid well. Fumigants are a class of VOCs and also a type of pesticide, but are discussed with VOCs in this report, because that is the constituent class group in which they were analyzed. Of the VOCs, 1or more were detected in 41 of the 77 gridwell samples (about 53 percent detection frequency). Four VOCs had detection frequencies greater than 10 percent: the fumigants 1,2-dichloropropane (1,2-DCP), 1,2,3-TCP, and $\mathrm{DBCP}$ and the gasoline oxygenate methyl tert-butyl ether (MTBE).

Pesticides include herbicides, insecticides, and fungicides and are used to control weeds, insects, fungi, and other pests in agricultural, urban, and suburban settings. Of the 115 pesticides analyzed, 12 were detected, and all concentrations were less than health-based benchmark concentrations (table $6 A$ ). Of the pesticides, 1 or more were detected in 33 of the 76 MACK study-unit grid well samples (about 43 percent detection frequency). The herbicide simazine was detected in more than 10 percent of the samples.

Pesticides can react with air, water, soil, sediments, or biota and form pesticide degradate compounds. Of the 114 pesticide degradates analyzed, 11 were detected, and all concentrations were less than health-based benchmark concentrations (table $6 B$ ). Of the pesticide degradates, 1 or more were detected in 42 of the 76 MACK study unit grid well samples (about 55 percent detection frequency). Each of the herbicide degradates chlorodiamino-s-triazine (CAAT), 2-chloro-6-ethylamino-4-amino-s-triazine (CEAT), demethyl norflurazon, and 2-chloro-4-isopropylamino-6-amino-striazine (CIAT or deethylatrazine) were detected in more than 10 percent of the samples.

Deethylatrazine and simazine are among the nation's most commonly detected pesticide compounds in groundwater (Gilliom and others, 2006).

\section{Inorganic Constituents}

Unlike the organic constituents, most of the inorganic constituents are naturally present in groundwater, although their concentrations can be influenced by human activities.
In the MACK study unit, 20 of the 23 trace elements and 1 of the 12 major and minor ions analyzed have regulatory or non-regulatory health-based benchmarks (table $3 C$ ). Of these 21 constituents with health-based benchmarks, 5 were detected at concentrations greater than benchmarks, 15 were only detected at concentrations less than their respective benchmarks, and 1 was not detected (tables 7 and 9). Arsenic concentrations greater than the MCL-US of $10 \mu \mathrm{g} / \mathrm{L}$ were detected in samples from 5 grid wells; manganese concentrations greater than the HBSL of $300 \mu \mathrm{g} / \mathrm{L}$ were detected in samples from 4 grid wells; a molybdenum concentration greater than the HBSL of $40 \mu \mathrm{g} / \mathrm{L}$ was detected in a sample from 1 grid well; uranium concentrations greater than the MCL-US of $30 \mu \mathrm{g} / \mathrm{L}$ were detected in samples from 17 grid wells; and vanadium concentrations greater than the NL-CA of $50 \mu \mathrm{g} / \mathrm{L}$ were detected in samples from 2 grid wells (table 7).

The nutrients nitrogen and phosphorus in groundwater can affect biological activity in aquifers and in surface-water bodies that receive groundwater discharge. Inorganic nitrogen can be present in the form of ammonia, nitrite, or nitrate, depending on the oxidation-reduction state of the groundwater. Groundwater samples from 21 grid wells had a nitrate concentration greater than the MCL-US of 10 milligrams per liter ( $\mathrm{mg} / \mathrm{L}$; table 8). All other nutrient concentrations in well samples from the MACK study unit were less than healthbased benchmarks.

The levels of certain trace elements, major-ion composition, and TDS content in groundwater can affect the aesthetic properties of water, such as taste, color, and odor, and the technical properties of water, such as scaling and staining. Although no adverse health effects are directly associated with these properties, they can reduce consumer satisfaction with the water or have economic effects. In addition to the SMCL-CAs for water-quality indicators, the State of California has also established non-regulatory benchmarks (SMCL-CAs) based on aesthetic properties rather than on human-health concerns for iron, manganese, silver, zinc, chloride, sulfate, and TDS. Manganese, silver, and zinc also have HBSLs, and the health-based, higher priority benchmarks are used for the comparison benchmarks instead of SMCLs in this study (table $3 C$ ). Iron and manganese are trace elements for which concentrations are affected by the oxidationreduction state of the groundwater. Precipitation of minerals containing iron or manganese can cause orange, brown, or black staining of surfaces.

Results for trace elements and major and minor ions with non-regulatory benchmarks set for aesthetic concerns showed that iron, sulfate, and TDS concentrations were each greater than established benchmarks in samples from two or more grid wells (tables 7, 9). Iron concentrations greater than SMCL-CA of $300 \mu \mathrm{g} / \mathrm{L}$ were detected in samples from three grid wells (table 7). Sulfate concentrations greater than the upper SMCL-CA of $500 \mathrm{mg} / \mathrm{L}$ were detected in samples from two grid wells and TDS concentrations greater than the upper SMCL-CA of $1,000 \mathrm{mg} / \mathrm{L}$ were detected in samples from five grid wells (table 9). 


\section{Perchlorate}

Perchlorate was classified as a constituent of special interest at the inception of the GAMA-PBP in 2003, because it had recently been detected in some drinking-water supplies in California, and the State was considering whether or not to pursue future regulation of this constituent. The State of California established an MCL-CA for perchlorate in 2007. For the second phase of the GAMA-PBP, perchlorate was grouped with the inorganic constituents. Perchlorate has natural and anthropogenic sources to groundwater. Perchlorate was detected in samples from 55 of the 76 grid wells for which it was analyzed, and all detections were at concentrations less than the MCL-CA of $6 \mu \mathrm{g} / \mathrm{L}$ (table 10).

\section{Radioactive Constituents}

Radioactivity is the release of energy or energetic particles during changes in the structure of the nucleus of an atom. Most radioactivity in groundwater comes from decay of the isotopes of uranium and thorium that are present in minerals in the sediments of the aquifer. The alpha and beta particles emitted during radioactive decay can be hazardous to human health because these energetic particles can damage cells.

Activity often is used instead of concentration for reporting the presence of radioactive constituents. The activity of radioactive constituents in groundwater is measured in units of picocuries per liter $(\mathrm{pCi} / \mathrm{L})$, and $1 \mathrm{pCi} / \mathrm{L}$ is approximately equal to two atoms decaying per minute. The number of atoms decaying is proportional to the number of particles or amount of energy emitted.

Samples for gross alpha-particle activity were collected from 73 (72-hour count) or 74 (30-day count) grid wells. The MCL-US of $15 \mathrm{pCi} / \mathrm{L}$ for gross alpha-particle activity applies to the adjusted gross alpha, not to the measured gross alpha (U.S. Environmental Protection Agency, 2012). Adjusted gross alpha is equal to the measured gross alpha minus the alpha activity attributable to uranium. Uranium activities were estimated from the measured uranium concentrations with a conversion factor of 0.67 (U.S. Environmental Protection Agency, 2000). Adjusted gross alpha-particle activity was greater than the MCL-US for one grid-well sample in the 72-hour count data (table 11). Samples were analyzed for gross beta radioactivity (72-hour and 30-day counts) from 74 grid wells, and activities of the samples measured in the 30 -day count were greater than the MCL-CA of $50 \mathrm{pCi} / \mathrm{L}$ for 8 grid wells.

Radon-222 activity was greater than the proposed MCL-US of 4,000 pCi/L in one grid-well sample (table 11).
The proposed MCL-US applies if a state or local water agency has an approved multimedia mitigation program to address radon levels in indoor air (U.S. Environmental Protection Agency, 1999). The proposed MCL-US for radon is considered a non-regulatory health-based benchmark for the purposes of this study.

\section{Geochemical and Age-Dating Tracers}

Geochemical and age-dating tracers can be used to help interpret hydrologic processes affecting groundwater quality (Clark and Fritz, 1997). Many such tracers are known, and the tracers selected for this study reported included stable-isotope ratios and radioactive isotopes.

The stable-isotope ratios of hydrogen $\left(\mathrm{d}^{2} \mathrm{H}\right)$ and oxygen $\left(\mathrm{d}^{18} \mathrm{O}\right)$ in water (table 12) help deduce the sources of groundwater recharge (see the "Notation" section of the appendix for a description of how stable-isotope ratios are reported). These isotopic ratios reflect the altitude, latitude, and temperature of precipitation as well as the extent of evaporation of the water in surface-water bodies or soils prior to infiltration into the aquifer.

Tritium activities (table 11) provide information about the age (time since recharge) of groundwater. Tritium is a shortlived radioactive isotope of hydrogen that is incorporated into the water molecule. Low levels of tritium are produced continuously by interaction of cosmic radiation with the Earth's atmosphere, and a large amount of tritium was produced as a result of atmospheric testing of nuclear weapons between 1952 and 1963. Thus, concentrations of tritium greater than background generally indicate the presence of water recharged after the early 1950s. Of the isotope-tracer constituents analyzed for this study, tritium is the only one with a health-based benchmark. All of the measured tritium activities in samples from the MACK study unit were less than the MCL-CA benchmark of $20,000 \mathrm{pCi} / \mathrm{L}$ (table 11), and nearly all were less than one-thousandth of the benchmark.

Carbon-14 (table 12), a radioactive isotope of carbon, is also an age-dating tracer. Low levels of carbon-14 are produced continuously by interaction of cosmic radiation with the Earth's atmosphere and incorporated into atmospheric carbon dioxide. Carbon dioxide dissolves in precipitation, surface water, and groundwater exposed to the atmosphere, thereby entering the hydrologic cycle. Because carbon-14 decays with a half-life of approximately 5,700 years, low activities of carbon-14 in water samples, relative to modern values, generally indicate groundwater that is several thousand years old or more. 


\section{Microbial Indicators}

Water is disinfected during drinking-water treatment to prevent diseases that can be spread by water-borne microbial constituents derived from human or animal wastes. The specific bacteria responsible for diseases generally are not measured for three reasons: (1) safety concerns related to cultivation of pathogenic organisms for analysis; (2) the time and cost incurred by a different test for each pathogen; and (3) the collection of large volumes of water required for analysis of pathogens, which are usually present at low concentrations. Non-pathogenic indicator organisms are more easily measured and can be used as surrogates for many different pathogens. Coliforms are bacteria that are naturally present in the environment and are used as an indicator that other, potentially harmful, bacteria could be present. Escherichia coli (E. coli) and enterococci are bacteria whose presence indicates that the water could be contaminated with human or animal wastes. The presence of microbial indicators does not mean that pathogenic bacteria are present, but indicates that the water system could be vulnerable to pathogen contamination from fecal sources.

The benchmarks for microbial indicators in groundwater include an MCL-US benchmark for E.coli and TT-US benchmarks for total coliforms and other potential fecal indicators, such as enterococci. Detections in public water systems at levels greater than the benchmarks may trigger requirements for re-testing for verification of results, assessments of the sanitary integrity of the groundwater source and distribution system, public notification, and additional disinfection (U.S. Environmental Protection Agency, 2016). Repeat sampling at a site was beyond the scope of this project.

Groundwater samples were analyzed for the presence or absence of three microbial indicators: E. coli, total coliform, and enterococci. The presence of $E$. coli was analyzed for in 73 grid wells and was not detected in any of the samples. The presence of total coliform (includes coliforms naturally present in soils as well as fecal coliform and E. coli) was detected in samples from 10 of the 72 grid wells for which it was analyzed, and the presence of enterococci was detected in samples from 5 of the 73 grid wells analyzed (table 13). Results of these analyses cannot be compared directly with benchmarks because the benchmarks were defined in terms of results from repeat sampling at sites. Two grid-well samples contained total coliform and enterococci.

\section{Future Work}

Subsequent reports are to focus on assessment of the data presented in this report by using a variety of statistical, qualitative, and quantitative approaches to evaluate the natural and human factors affecting groundwater quality in the MACK study unit. Additional water-quality data may be compiled, evaluated, and used in the assessments in combination with the data that are presented in this report.

\section{Summary}

Groundwater quality in the 2,390-square-mile Madera/ Chowchilla-Kings Shallow Aquifer (MACK) study unit was investigated by the U.S. Geological Survey (USGS) from August 2013 to April 2014 in cooperation with the California State Water Resources Control Board (SWRCB) as part of the SWRCB Groundwater Ambient Monitoring and Assessment (GAMA) Program Priority Basin Project. The GAMA Priority Basin Project (GAMA-PBP) was established to provide comprehensive assessment and monitoring of the quality of groundwater used for drinking water supplies in the State. The MACK study unit consisted of the Madera, Chowchilla, and Kings subbasins of the San Joaquin Valley groundwater basin.

The GAMA MACK study was designed to provide a statistically unbiased assessment of untreated groundwater quality in the shallow aquifer system. The shallow-aquifer system corresponds to the part of the aquifer system in the study unit that is used by domestic wells, and generally is shallower than the part of the aquifer system that is used by public-supply wells.

Most of the wells sampled for this study were private domestic wells. Unlike groundwater from public-supply wells, the groundwater from private domestic wells is not regulated for quality in California and is rarely analyzed for water-quality constituents. To provide some context for the results, however, concentrations of constituents measured in the untreated groundwater were compared to regulatory and non-regulatory health-based benchmarks established by the U.S. Environmental Protection Agency (EPA), the State of California, and the USGS and non-regulatory benchmarks established for aesthetic concerns by the State of California.

The MACK study included an assessment of the groundwater quality of samples from 77 grid wells in Fresno, Kings, Madera, Merced, and Tulare counties. Wells were selected by using a randomized, grid-based approach to achieve a statistically unbiased representation of groundwater in shallow-aquifer systems used primarily for domestic drinking-water supplies.

Groundwater samples were analyzed for field waterquality indicators, organic constituents, inorganic constituents, perchlorate, radioactive constituents, geochemical and age-dating tracers, and microbial indicators. Isotopes were measured to provide a dataset that can be used to interpret the sources and ages of the sampled groundwater. In total, 389 constituents and water-quality indicators were measured for this study. This report describes the sampling, analytical, and quality-assurance methods used in the study and presents the results of the chemical analyses of the groundwater samples. 
In the 77 grid-well samples, 3 detections of 1,2-dibromo-3-chloropropane (DBCP) were greater than the U.S. Environmental Protection Agency maximum contaminant level (MCL-US), 1 detection of 1,2-dibromoethane (EDB) was greater than the MCL-US, 5 detections of arsenic were greater than the MCL-US, 4 detections of manganese were greater than the USGS Health-Based Screening Level (HBSL), 1 detection of molybdenum was greater than the HBSL, 17 detections of uranium were greater than the MCL-US, 2 detections of vanadium were greater than the California notification level (NL-CA), 21 detections of nitrate were greater than the MCL-US, 1 detection of radon-222 was greater than the proposed MCL-US, 1 detection of gross alpha radioactivity 72 -hour count was greater than the MCL-US for adjusted gross alpha, and 8 detections of gross beta radioactivity 30 -day count were greater than the California maximum contaminant level (MCL-CA). The following constituents were measured at concentrations greater than the State of California secondary maximum contaminant level (SMCL-CA): three detections of iron, two detections of sulfate (greater than the upper benchmark level), and five detections of total dissolved solids (TDS, greater than the upper benchmark level). The $\mathrm{pH}$ was outside of the U.S. Environmental Protection Agency secondary maximum contaminant level (SMCL-US) range in three samples, and the specific conductance was greater than the SMCL-US in five samples. The presence of total coliform (including fecal coliform and Escherichia coli; or E. coli) was detected in samples from 10 of the 72 grid wells for which it was analyzed, and the presence of enterococci was detected in samples from 5 of the 73 grid wells analyzed. Samples from two grid wells contained both total coliform and enterococci.

\section{References Cited}

Belitz, Kenneth, Dubrovsky, N.M., Burow, Karen, Jurgens, B.C., and Johnson, Tyler, 2003, Framework for a ground-water quality monitoring and assessment program for California: U.S. Geological Survey WaterResources Investigations Report 2003-4166, 78 p., https://pubs.er.usgs.gov/publication/wri034166.

Belitz, Kenneth, Fram, M.S., and Johnson, T.D., 2015, Metrics for assessing the quality of groundwater used for public supply, CA, USA-Equivalent-population and area: Environmental Science and Technology, v. 49, no. 14, p. 8330-8338, http://dx.doi.org/10.1021/acs.est.5b00265.

Bertoldi, G.L., Johnston, R.H., and Evenson, K.D., 1991, Ground water in the Central Valley, California-A Summary Report: U.S. Geological Survey Professional Paper 1401-A, 44 p., https://pubs.er.usgs.gov/publication/pp1401A.
Burow, K.R., Weissmann, G.S., Miller, R.D., and Placzek, Gary, 1997, Hydrogeologic facies characterization of an alluvial fan near Fresno, California, using geophysical techniques: U.S. Geological Survey Open-File Report 97-46, 15 p., https://pubs.er.usgs.gov/publication/ofr9746.

Burow, K.R., Shelton, J.L., and Dubrovsky, N.M., 2008, Regional nitrate and pesticide trends in ground water in the eastern San Joaquin Valley, California: Journal of Environmental Quality, v. 37, no. 5, Supplement, p. S-249-S-263, http://dx.doi.org/10.2134/jeq2007.0061.

Burton, C.A., and Belitz, Kenneth, 2008, Ground-water quality data in the southeast San Joaquin Valley, 20052006 - Results from the California GAMA Program: U.S. Geological Survey Data Series 351, 103 p., https://pubs.er.usgs.gov/publication/ds351.

Burton, C.A., Shelton, J.L., and Belitz, Kenneth, 2012, Status and understanding of groundwater quality in the two southern San Joaquin Valley study units, 2005-2006California GAMA Priority Basin Project: U.S. Geological Survey Scientific Investigations Report 2011-5218, 106 p., https://pubs.er.usgs.gov/publication/sir20115218.

California Department of Water Resources, 2003, California's groundwater update 2003: California Department of Water Resources Bulletin 118, 246 p., accessed January 28, 2015, at http://www.water.ca.gov/groundwater/bulletin118/ update_2003.cfm.

California Department of Water Resources, 2006, California's groundwater-Individual basin descriptions-Tulare Lake Hydrologic Region, San Joaquin Valley Groundwater Basin, Kings Subbasin: California Department of Water Resources Bulletin 118, accessed January 28, 2015, at http://www.water.ca.gov/pubs/groundwater/bulletin_118/ basindescriptions/5-22.08.pdf.

California Department of Water Resources, 2008a, California's groundwater-Individual basin descriptions-San Joaquin River Hydrologic Region, San Joaquin Valley Groundwater Basin, Chowchilla Subbasin: California Department of Water Resources Bulletin 118, accessed January 28, 2015, at http://www.water.ca.gov/pubs/groundwater/bulletin_118/ basindescriptions/5-22.05.pdf.

California Department of Water Resources, 2008b, California's groundwater-Individual basin descriptionsSan Joaquin River Hydrologic Region, San Joaquin Valley Groundwater Basin, Madera Subbasin: California Department of Water Resources Bulletin 118, accessed January 28, 2015, at http://www.water.ca.gov/pubs/ groundwater/bulletin_118/basindescriptions/5-22.06.pdf. 
California State Water Resources Control Board, 2003, Report to the Governor and Legislature-A comprehensive groundwater quality monitoring program for California: Assembly Bill 599, 121 p., accessed January 28, 2015, at http://www.waterboards.ca.gov/gama/docs/final_ab_599_ rpt_to_legis_7_31_03.pdf.

California State Water Resources Control Board, Division of Drinking Water, 2010, Drinking water notification levels and response levels-An overview, accessed January 28, 2015, at http://www.waterboards.ca.gov/drinking_water/ certlic/drinkingwater/documents/notificationlevels/ notificationlevels.pdf.

California State Water Resources Control Board, 2013, California drinking water-related laws-Drinking water-related statutes and regulations, Title 22 Code of Regulations: California Department of Public Health, accessed January 28, 2015, at http://www.waterboards. ca.gov/drinking_water/certlic/drinkingwater/Lawbook. shtml.

Clark, I.D., and Fritz, Peter, 1997, Environmental isotopes in hydrogeology: Boca Raton, Florida, CRC Press LLC, 328 p.

Davis, G.H., Green, J.H., Olmsted, F.H., and Brown, D.W., 1959, Ground-water conditions and storage capacity in the San Joaquin Valley, California: U.S. Geological Survey Water Supply Paper 1469, 287 p., https://pubs.er.usgs.gov/publication/wsp1469.

Davis, T.A., Olsen, L.D., Fram, M.S., and Belitz, Kenneth, 2014, Updated study reporting levels (SRLs) for traceelement data collected for the California Groundwater Ambient Monitoring and Assessment (GAMA) Priority Basin Project, October 2009-March 2013: U.S. Geological Survey Scientific Investigations Report 2014-5105, 52 p., http://dx.doi.org/10.3133/sir20145105.

Faunt, C.C., ed., 2009, Groundwater availability of the Central Valley Aquifer, California: U.S. Geological Survey Professional Paper 1766, 227 p., https://pubs.er.usgs.gov/publication/pp1766.

Gilliom, R.J., Barbash, J.E., Crawford, C.G., Hamilton, P.A., Martin, J.D., Nakagaki, Naomi, Nowell, L.H., Scott, J.C., Stackelberg, P.E., Thelin, G.P., and Wolock, D.M., 2006, The quality of our nation's waters-Pesticides in the nation's streams and ground water, 1992-2001: U.S. Geological Survey Circular 1291, 173 p., https://pubs.er.usgs.gov/publication/cir1291.
Gronberg, J.A., Dubrovsky, N.M., Kratzer, C.R., Domagalski, J.L., Brown, L.R., and Burow, K.R., 1998, Environmental setting of the San Joaquin-Tulare basins, California: U.S. Geological Survey WaterResources Investigations Report 97-4205, 45 p., https://pubs.er.usgs.gov/publication/wri974205.

Hirsch, R.M., Alley, W.M., and Wilber, W.G., 1988, Concepts for a National Water-Quality Assessment Program: U.S. Geological Survey Circular 1021, 42 p., https://pubs.er.usgs.gov/publication/cir1021.

Johnson, T.D., and Belitz, Kenneth, 2014, California groundwater units: U.S. Geological Survey Data Series 796, 34 p., http://dx.doi.org/10.3133/ds796.

Johnson, T.D., and Belitz, Kenneth, 2015, Identifying the location and population served by domestic wells in California: Journal of Hydrology—Regional Studies, v. 3, p. 31-86, http://dx.doi.org/10.1016/j.ejrh.2014.09.002.

Kenny, J.F., Barber, N.L., Hutson, S.S., Linsey, K.S., Lovelace, J.K., and Maupin, M.A., 2009, Estimated use of water in the United States in 2005: U.S. Geological Survey Circular 1344, 52 p., https://pubs.er.usgs.gov/publication/cir1344.

Koterba, M.T., Wilde, F.D., and Lapham, W.W., 1995, Groundwater data-collection protocols and procedures for the National Water-Quality Assessment Program - Collection and documentation of water-quality samples and related data: U.S. Geological Survey Open-File Report 95-399, 114 p., https://pubs.er.usgs.gov/publication/ofr95399.

Kulongoski, J.T., and Belitz, Kenneth, 2004, Groundwater ambient monitoring and assessment program: U.S. Geological Survey Fact Sheet 2004-3088, 2 p., https://pubs.er.usgs.gov/publication/fs20043088.

Scott, J.C., 1990, Computerized stratified random siteselection approaches for design of a ground-water-quality sampling network: U.S. Geological Survey WaterResources Investigations Report 90-4101, 109 p., https://pubs.er.usgs.gov/publication/wri904101.

Shelton, J.L., Fram, M.S., and Belitz, Kenneth, 2009, Groundwater-quality data for the Madera-Chowchilla study unit, 2008-Results from the California GAMA program: U.S. Geological Survey Data Series 455, 81 p., https://pubs.er.usgs.gov/publication/ds455. 
Shelton, J.L., Fram, M.S., Belitz, Kenneth, and Jurgens, B.C., 2013, Status and understanding of groundwater quality in the Madera-Chowchilla study unit, 2008-California GAMA Priority Basin Project: U.S. Geological Survey Scientific Investigations Report 2012-5094, 86 p., https://pubs.er.usgs.gov/publication/sir20125094.

State of California, 2001a, An act to add Part 2.76 (commencing with Section 10780) to Division 6 of the Water Code, relating to water: Assembly Bill No. 599, chapter 522, accessed August 13, 2015, at http://www.waterboards.ca.gov/water_issues/programs/ gama/docs/ab_599_bill_20011005_chaptered.pdf.

State of California, 2001b, Groundwater Quality Monitoring Act of 2001-California Water Code, part 2.76, Sections 10780-10782.3, accessed August 13, 2015, at http://leginfo.legislature.ca.gov/faces/codes_displayText.xht ml lawCode $=$ WAT $\&$ division $=6 . \&$ title $=\&$ part $=2.76 . \&$ chapt er $=$ \&article $=$.

Toccalino, P.L., Norman, J.E., Booth, N.L, Thompson, J.L., and Zogorski, J.S., 2012, Health-based screening levels (HBSL) for evaluating water-quality data: U.S. Geological Survey, National Water-Quality Assessment Program, accessed February 19, 2014, at http://water.usgs.gov/nawqa/HBSL/.

U.S. Census Bureau, 1990, Census of population and housing, summary tape file 3A, accessed June 6, 2013, at ftp://ftp2.census.gov/census_1990/.

U.S. Environmental Protection Agency, 1999, National primary drinking water regulations, Radon-222: Federal Register, v. 64, no. 211, p. 59, 245-59, 294.
U.S. Environmental Protection Agency, 2000, National primary drinking water regulations; Radionuclides; Final Rule 40 CFR Parts 9, 141, and 142: Washington, D.C., Federal Register, v. 65, no. 236, p. 76708-76753, accessed August 9, 2016, at https:/www.federalregister.gov/ articles/2000/12/07/00-30421/national-primary-drinkingwater-regulations-radionuclides-final-rule.

U.S. Environmental Protection Agency, 2012, 2012 Edition of the drinking water standards and health advisories, accessed January 28, 2015, at http:/water.epa.gov/action/advisories/ drinking/upload/dwstandards2012.pdf.

U.S. Environmental Protection Agency, 2013, Human health benchmarks for pesticides: Updated 2013 technical document: U.S. Environmental Protection Agency, Office of Water and Office of Pesticide Programs EPA 820-R-13010, August 2013, 5 p., accessed April 10, 2015, at http://www.epa.gov/pesticides/hhbp/HHBP-tech.pdf.

U.S. Environmental Protection Agency, 2016, National Primary Drinking Water Regulations-Maximum Contaminant Levels and Maximum Residual Disinfectant Levels: Code of Federal Regulations, Title 40, Part 141, Subpart G, accessed July 2016, at http://www.ecfr.gov/cgibin/text-idx?SID=0ee8e46e25bd39f1577864c8376c8607\& $\mathrm{mc}=$ true $\&$ node $=\mathrm{pt} 40.25 .141 \& \operatorname{rgn}=\operatorname{div} 5 \# \mathrm{sp} 40.25 .141 . \mathrm{g}$.

U.S. Geological Survey, [variously dated], National field manual for the collection of water-quality data: U.S. Geological Survey Techniques of Water-Resources Investigations, book 9, chap. A1-A9, accessed January 28, 2015, at http://water.usgs.gov/owq/FieldManual/. 
Table 1. Identification, sampling, construction and water-level information for wells sampled in the Madera/Chowchilla-Kings Shallow Aquifer study unit, Groundwater Ambient Monitoring and Assessment (GAMA) Priority Basin Project, California, August 2013 to April 2014.

[Land-surface datum (LSD) is a datum plane that is approximately at land surface at each well. The altitude of the LSD is described in feet above the North American Vertical Datum 1988 (NAVD 88). GAMA well identification numbers: S3-MACK-M, Madera/Chowchilla-Kings Shallow Aquifer Madera/ Chowchilla study-area grid well; S3-MACK-K, Madera/Chowchilla-Kings Shallow Aquifer Kings study-area grid well. Abbreviations: ft, feet; mm/dd/yyyy, month/day/year; na, not available; USGS, U.S. Geological Survey; >, greater than]

\begin{tabular}{|c|c|c|c|c|c|c|c|}
\hline \multicolumn{4}{|c|}{ Sampling information } & \multicolumn{3}{|c|}{ Construction information } & \multirow{2}{*}{$\begin{array}{c}\text { Depth to } \\
\text { water level } \\
\text { (ft below LSD) }\end{array}$} \\
\hline $\begin{array}{c}\text { GAMA well } \\
\text { identification } \\
\text { number }\end{array}$ & $\begin{array}{l}\text { Date sampled } \\
\text { (mm/dd/yyyy) }\end{array}$ & Well type & $\begin{array}{c}\text { Altitude of LSD } \\
\text { (ft above } \\
\text { NAVD 88) }\end{array}$ & $\begin{array}{c}\text { Well depth } \\
\text { (ft below LSD) }\end{array}$ & $\begin{array}{l}\text { Depth to top of } \\
\text { highest perforation } \\
\text { (ft below LSD) }\end{array}$ & $\begin{array}{l}\text { Depth to bottom of } \\
\text { lowest perforation } \\
\text { (ft below LSD) }\end{array}$ & \\
\hline \multicolumn{8}{|c|}{ Madera/Chowchilla-Kings Shallow Aquifer study-unit grid wells (77 wells sampled) } \\
\hline \multicolumn{8}{|c|}{ Madera/Chowchilla study-area grid wells } \\
\hline S3-MACK-M03 & $4 / 10 / 2014$ & Production & 172 & 200 & na & na & 118.83 \\
\hline S3-MACK-M04 & $4 / 10 / 2014$ & Production & 153 & 340 & 300 & 340 & 144.52 \\
\hline S3-MACK-M05 & $11 / 20 / 2013$ & Production & 137 & 260 & 160 & 260 & na \\
\hline S3-MACK-M06 & $11 / 19 / 2013$ & Production & 156 & 117 & 117 & 117 & 44.38 \\
\hline S3-MACK-M10 & $12 / 4 / 2013$ & Production & 214 & 300 & 240 & 300 & 150 \\
\hline S3-MACK-M11 & $12 / 3 / 2013$ & Production & 232 & 300 & 240 & 300 & 102.59 \\
\hline S3-MACK-M12 & $3 / 18 / 2014$ & Production & 244 & 253 & 213 & 253 & 68.01 \\
\hline S3-MACK-M13 & $12 / 2 / 2013$ & Production & 232 & 280 & 280 & 280 & 132.33 \\
\hline S3-MACK-M14 & $12 / 11 / 2013$ & Production & 232 & 400 & na & na & na \\
\hline S3-MACK-M15 & $12 / 5 / 2013$ & Production & 217 & 420 & 360 & 420 & 229.95 \\
\hline S3-MACK-M16 & $11 / 18 / 2013$ & Production & 206 & 300 & 160 & 300 & 86.08 \\
\hline S3-MACK-M17 & $3 / 20 / 2014$ & Production & 198 & 300 & 180 & 300 & 209.66 \\
\hline S3-MACK-M23 & $12 / 9 / 2013$ & Production & 287 & 165 & 130 & 140 & 34.70 \\
\hline S3-MACK-M24 & $12 / 9 / 2013$ & Production & 412 & 320 & 200 & 320 & na \\
\hline S3-MACK-M25 & $3 / 19 / 2014$ & Production & 353 & 310 & na & na & 281.45 \\
\hline S3-MACK-M26 & $12 / 10 / 2013$ & Production & 380 & 285 & na & na & ${ }^{1}>200$ \\
\hline S3-MACK-M27 & $12 / 10 / 2013$ & Production & 353 & 369 & na & na & 282.87 \\
\hline S3-MACK-M28 & 4/9/2014 & Production & 340 & 340 & 240 & 340 & 140.02 \\
\hline
\end{tabular}


Table 1. Identification, sampling, construction and water-level information for wells sampled in the Madera/Chowchilla-Kings Shallow Aquifer study unit, Groundwater Ambient Monitoring and Assessment (GAMA) Priority Basin Project, California, August 2013 to April 2014.-Continued

[Land-surface datum (LSD) is a datum plane that is approximately at land surface at each well. The altitude of the LSD is described in feet above the North American Vertical Datum 1988 (NAVD 88). GAMA well identification numbers: S3-MACK-M, Madera/Chowchilla-Kings Shallow Aquifer Madera/ Chowchilla study-area grid well; S3-MACK-K, Madera/Chowchilla-Kings Shallow Aquifer Kings study-area grid well. Abbreviations: ft, feet; mm/dd/yyyy, month/day/year; na, not available; USGS, U.S. Geological Survey; >, greater than]

\begin{tabular}{|c|c|c|c|c|c|c|c|}
\hline \multicolumn{4}{|c|}{ Sampling information } & \multicolumn{3}{|c|}{ Construction information } & \multirow[b]{2}{*}{$\begin{array}{c}\text { Depth to } \\
\text { water level } \\
\text { (ft below LSD) }\end{array}$} \\
\hline $\begin{array}{c}\text { GAMA well } \\
\text { identification } \\
\text { number }\end{array}$ & $\begin{array}{l}\text { Date sampled } \\
\text { (mm/dd/yyyy) }\end{array}$ & Well type & $\begin{array}{c}\text { Altitude of LSD } \\
\text { (ft above } \\
\text { NAVD 88) }\end{array}$ & $\begin{array}{c}\text { Well depth } \\
\text { (ft below LSD) }\end{array}$ & $\begin{array}{l}\text { Depth to top of } \\
\text { highest perforation } \\
\text { (ft below LSD) }\end{array}$ & $\begin{array}{l}\text { Depth to bottom of } \\
\text { lowest perforation } \\
\text { (ft below LSD) }\end{array}$ & \\
\hline \multicolumn{8}{|c|}{ Madera/Chowchilla-Kings Shallow Aquifer study-unit grid wells (77 wells sampled)_Continued } \\
\hline \multicolumn{8}{|c|}{ Kings study-area grid wells } \\
\hline S3-MACK-K01 & $1 / 7 / 2014$ & Production & 218 & 55 & 35 & 55 & 8.99 \\
\hline S3-MACK-K03 & $1 / 28 / 2014$ & Production & 201 & 332 & na & na & 214.56 \\
\hline S3-MACK-K04 & $9 / 16 / 2013$ & Production & 188 & 300 & 240 & 300 & na \\
\hline S3-MACK-K05 & $1 / 30 / 2014$ & Production & 178 & 260 & 200 & 260 & na \\
\hline S3-MACK-K06 & $1 / 29 / 2014$ & Production & 176 & 220 & 200 & 220 & ${ }^{1}>154$ \\
\hline S3-MACK-K10 & $9 / 18 / 2013$ & Production & 213 & 212 & 152 & 212 & na \\
\hline S3-MACK-K11 & $9 / 12 / 2013$ & Production & 205 & 300 & 300 & 300 & ${ }^{1}>76$ \\
\hline S3-MACK-K12 & $1 / 7 / 2014$ & Production & 210 & 400 & 280 & 400 & 203.33 \\
\hline S3-MACK-K13 & $1 / 27 / 2014$ & Production & 217 & 285 & 265 & 285 & 177.50 \\
\hline S3-MACK-K14 & $1 / 27 / 2014$ & Production & 228 & 173 & na & na & na \\
\hline S3-MACK-K15 & $2 / 3 / 2014$ & Production & 248 & 200 & 160 & 200 & 113.95 \\
\hline S3-MACK-K16 & $2 / 3 / 2014$ & Production & 261 & 150 & na & na & 103.61 \\
\hline S3-MACK-K17 & $2 / 4 / 2014$ & Production & 248 & 209 & 160 & 209 & ${ }^{1}>160$ \\
\hline S3-MACK-K23 & $1 / 9 / 2014$ & Monitoring & 283 & 166 & 146 & 156 & 93.00 \\
\hline S3-MACK-K24 & $8 / 22 / 2013$ & Production & 261 & 180 & 140 & 180 & 86.86 \\
\hline S3-MACK-K25 & $2 / 26 / 2014$ & Production & 280 & 200 & 160 & 200 & na \\
\hline S3-MACK-K26 & $2 / 4 / 2014$ & Production & 272 & 156 & 116 & 156 & 97.01 \\
\hline S3-MACK-K27 & $3 / 4 / 2014$ & Production & 287 & 140 & 120 & 140 & na \\
\hline S3-MACK-K28 & $3 / 18 / 2014$ & Production & 303 & 140 & 120 & 140 & 42.00 \\
\hline S3-MACK-K29 & $3 / 17 / 2014$ & Production & 302 & 160 & 160 & 160 & na \\
\hline S3-MACK-K30 & $2 / 6 / 2014$ & Production & 311 & 180 & 140 & 180 & na \\
\hline
\end{tabular}


Table 1. Identification, sampling, construction and water-level information for wells sampled in the Madera/Chowchilla-Kings Shallow Aquifer study unit, Groundwater Ambient Monitoring and Assessment (GAMA) Priority Basin Project, California, August 2013 to April 2014.-Continued

[Land-surface datum (LSD) is a datum plane that is approximately at land surface at each well. The altitude of the LSD is described in feet above the North American Vertical Datum 1988 (NAVD 88). GAMA well identification numbers: S3-MACK-M, Madera/Chowchilla-Kings Shallow Aquifer Madera/ Chowchilla study-area grid well; S3-MACK-K, Madera/Chowchilla-Kings Shallow Aquifer Kings study-area grid well. Abbreviations: ft, feet; mm/dd/yyyy, month/day/year; na, not available; USGS, U.S. Geological Survey; >, greater than]

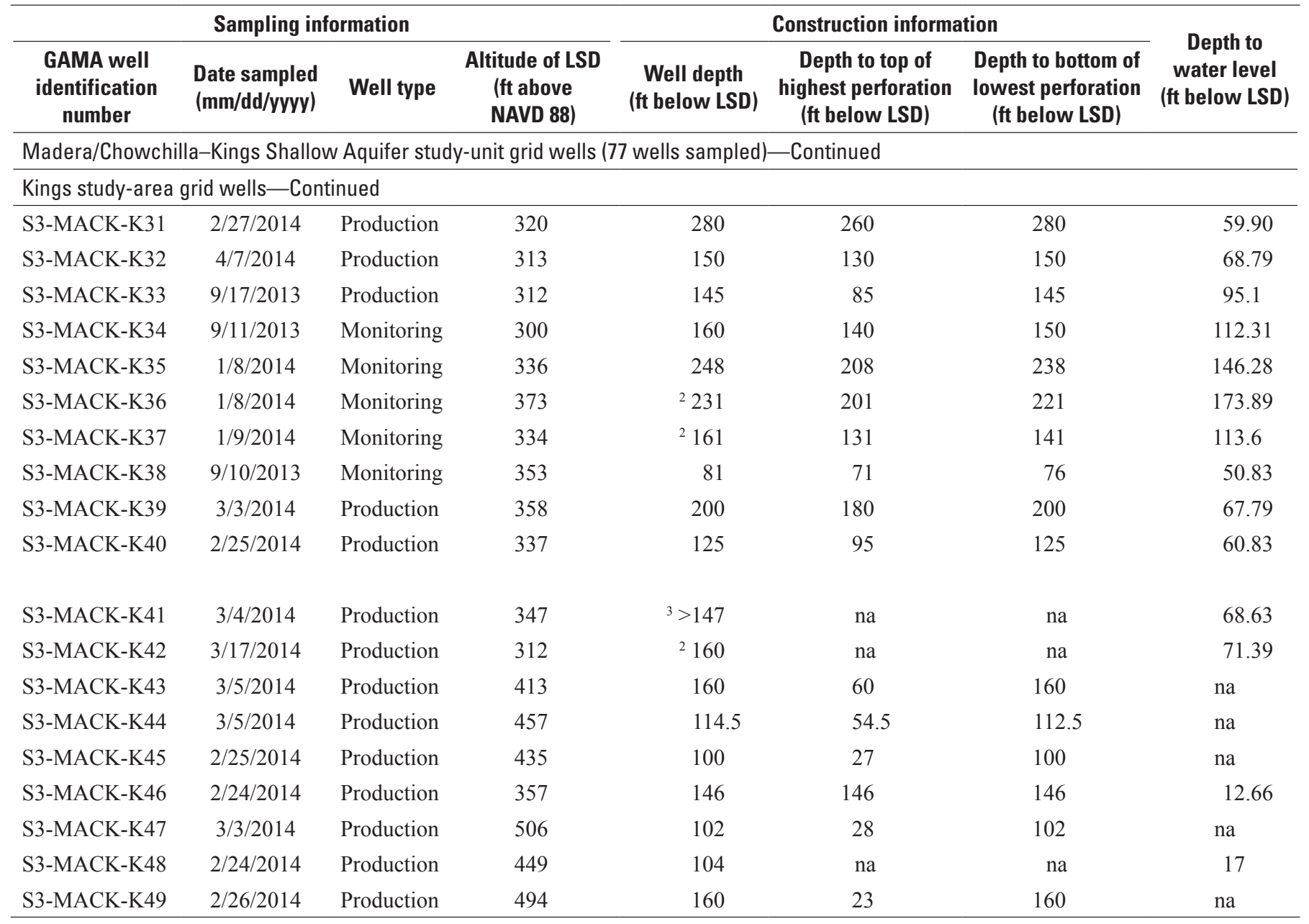

${ }^{1}$ Depth to water level is greater than depth of obstruction noted when USGS attempted to measure.

${ }^{2}$ Measured depth differs from well completion report depth noted, indicating well could have partially filled in with sediment (-K36, USGS measured well depth $206 \mathrm{ft}$; -K37, USGS measured well depth $146 \mathrm{ft}$; -K42, owner reported pump company measured depth $100 \mathrm{ft}$ in 2013 ).

${ }^{3}$ Well depth is greater than pump depth noted. 
Table 2. Classes of chemical constituents and field water-quality indicators measured for the Madera/Chowchilla-Kings Shallow Aquifer study unit, Groundwater Ambient Monitoring and Assessment (GAMA) Priority Basin Project, California, August 2013 to April 2014.

[na, not applicable]

\begin{tabular}{|c|c|c|}
\hline Constituent classes & Constituent list table & Results table \\
\hline \multicolumn{3}{|c|}{ Field water-quality indicators } \\
\hline Dissolved oxygen, temperature, $\mathrm{pH}$, and specific conductance & na & 4 \\
\hline Field alkalinity, bicarbonate, and carbonate & na & 4 \\
\hline \multicolumn{3}{|l|}{ Organic constituents } \\
\hline Volatile organic compounds (VOCs) & $3 A$ & 5 \\
\hline Pesticides and pesticide degradates & $3 B$ & $6 A, B$ \\
\hline \multicolumn{3}{|l|}{ Inorganic constituents } \\
\hline Trace elements & $3 C$ & 7 \\
\hline Nutrients & $3 C$ & 8 \\
\hline Major and minor ions, silica, and total dissolved solids (TDS) & $3 C$ & 9 \\
\hline Laboratory alkalinity, bicarbonate, and carbonate & $3 C$ & 4 \\
\hline Perchlorate & $3 C$ & 10 \\
\hline Radon-222 & $3 C$ & 11 \\
\hline Gross alpha and gross beta radioactivity (72-hour and 30 -day) & $3 C$ & 11 \\
\hline \multicolumn{3}{|l|}{ Isotopic tracers } \\
\hline Tritium & $3 C$ & 11 \\
\hline Stable isotopes of hydrogen and oxygen in water & $3 C$ & 12 \\
\hline Stable isotopes of carbon in dissolved inorganic carbon and carbon-14 abundance & $3 C$ & 12 \\
\hline Stable isotopes of nitrogen and oxygen in nitrate & $3 C$ & 12 \\
\hline Dissolved standard gases & na & none $^{1}$ \\
\hline Dissolved noble gases and helium isotope ratios & na & none $^{1}$ \\
\hline \multicolumn{3}{|l|}{ Microbial indicators } \\
\hline Escherichia coli (E. coli) & $3 D$ & 13 \\
\hline Total coliform & $3 D$ & 13 \\
\hline Enterococci & $3 D$ & 13 \\
\hline
\end{tabular}


Table 3A. Volatile organic compounds (VOCs), primary uses or sources, comparison benchmarks, and reporting information for the U.S. Geological Survey (USGS) National Water Quality Laboratory (NWQL) Schedules 4436 and 4437.

[The five-digit USGS parameter code is used to uniquely identify a specific constituent or property. Laboratory reporting level (LRL), interim reporting level (IRL), benchmark type, and benchmark level as of April 30, 2014. Benchmark types: Maximum contaminant level benchmarks are listed as MCL-US when the MCL-US and MCL-CA are identical, and as MCL-CA when the MCL-CA is lower than the MCL-US or no MCL-US exists. HBSL, USGS Health Based Screening Level; MCL-CA, State of California maximum contaminant level; MCL-US, U.S. Environmental Protection Agency (EPA) maximum contaminant level; MCL-HI, Hawaii Department of Health maximum contaminant level; NL-CA, State of California notification level; HAL-US, EPA lifetime health advisory level; RSD5-US, EPA risk specific dose at a risk factor of $10^{-5}$. Abbreviations: CAS, Chemical Abstracts Service; CASRN, Chemical Abstracts Service Registry Number; D, detected in groundwater samples (table 5); na, not available; THM, trihalomethane; $\mu \mathrm{g} / \mathrm{L}$, micrograms per liter; -, not detected]

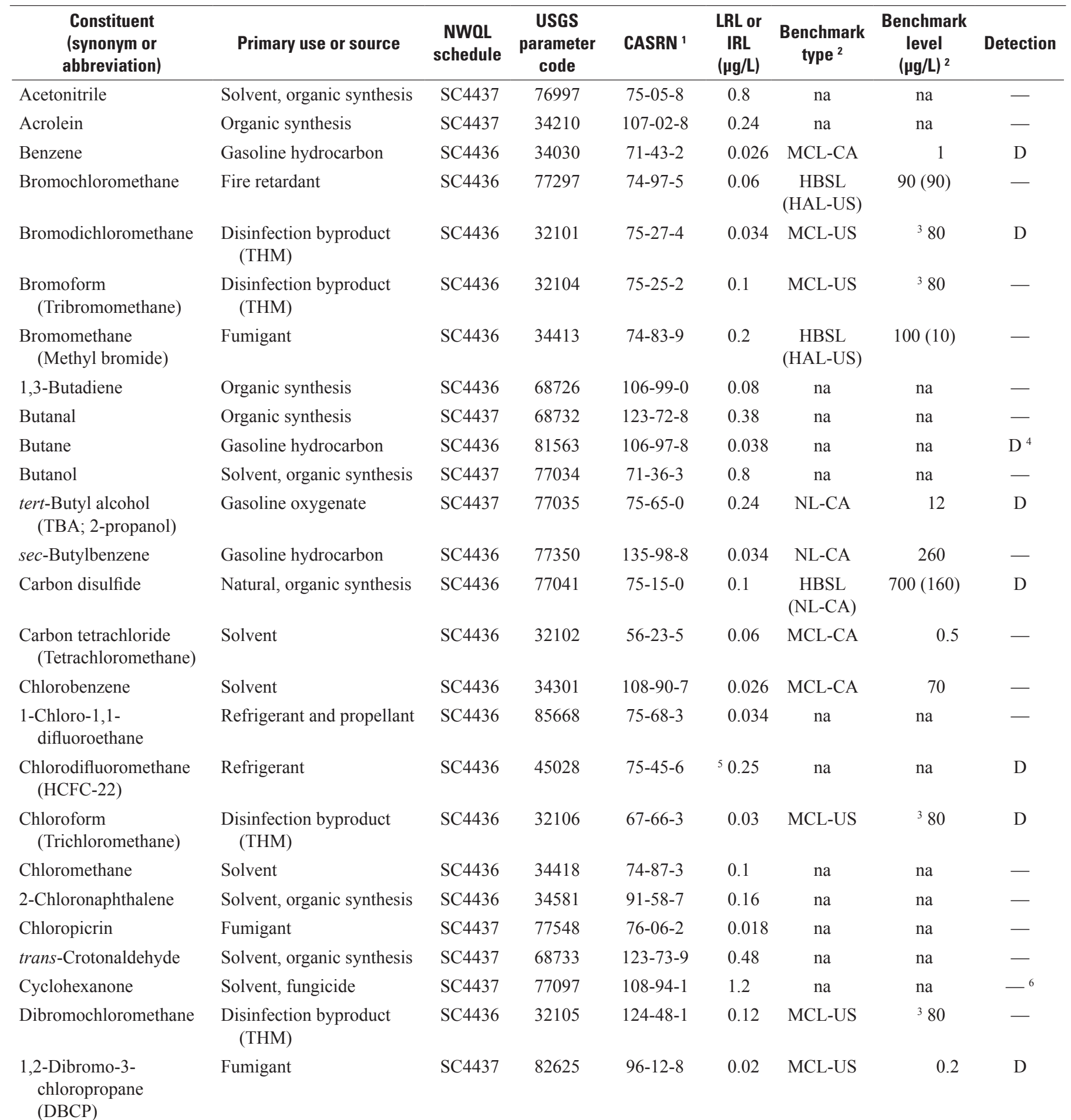


Table 3A. Volatile organic compounds (VOCs), primary uses or sources, comparison benchmarks, and reporting information for the U.S. Geological Survey (USGS) National Water Quality Laboratory (NWQL) Schedules 4436 and 4437.—Continued

[The five-digit USGS parameter code is used to uniquely identify a specific constituent or property. Laboratory reporting level (LRL), interim reporting level (IRL), benchmark type, and benchmark level as of April 30, 2014. Benchmark types: Maximum contaminant level benchmarks are listed as MCL-US when the MCL-US and MCL-CA are identical, and as MCL-CA when the MCL-CA is lower than the MCL-US or no MCL-US exists. HBSL, USGS Health Based Screening Level; MCL-CA, State of California maximum contaminant level; MCL-US, U.S. Environmental Protection Agency (EPA) maximum contaminant level; MCL-HI, Hawaii Department of Health maximum contaminant level; NL-CA, State of California notification level; HAL-US, EPA lifetime health advisory level; RSD5-US, EPA risk specific dose at a risk factor of $10^{-5}$. Abbreviations: CAS, Chemical Abstracts Service; CASRN, Chemical Abstracts Service Registry Number; D, detected in groundwater samples (table 5); na, not available; THM, trihalomethane; $\mu \mathrm{g} / \mathrm{L}$, micrograms per liter; -, not detected]

\begin{tabular}{|c|c|c|c|c|c|c|c|c|}
\hline $\begin{array}{l}\text { Constituent } \\
\text { (synonym or } \\
\text { abbreviation) }\end{array}$ & Primary use or source & $\begin{array}{l}\text { NWOL } \\
\text { schedule }\end{array}$ & $\begin{array}{c}\text { USGS } \\
\text { parameter } \\
\text { code }\end{array}$ & CASRN ${ }^{1}$ & $\begin{array}{l}\text { LRL or } \\
\text { IRL } \\
(\mu \mathrm{g} / \mathrm{L})\end{array}$ & $\begin{array}{l}\text { Benchmark } \\
\text { type }^{2}\end{array}$ & $\begin{array}{c}\text { Benchmark } \\
\text { level } \\
(\mu \mathrm{g} / \mathrm{L})^{2}\end{array}$ & Detection \\
\hline 2,6-Di-tert-butylphenol & Gasoline hydrocarbon & SC4436 & 61701 & $128-39-2$ & 0.16 & na & na & - \\
\hline $\begin{array}{l}\text { 1,2-Dibromoethane } \\
\text { (EDB) }\end{array}$ & Fumigant & SC4437 & 77651 & $106-93-4$ & 0.004 & MCL-US & 0.05 & $\mathrm{D}$ \\
\hline 1,2-Dichlorobenzene & Solvent & SC4436 & 34536 & $95-50-1$ & 0.028 & MCL-US & 600 & - \\
\hline $\begin{array}{l}\text { 1,1-Dichloroethane } \\
\text { (1,1-DCA) }\end{array}$ & Solvent & SC4436 & 34496 & $75-34-3$ & 0.044 & MCL-CA & 5 & $\mathrm{D}$ \\
\hline $\begin{array}{l}\text { 1,2-Dichloroethane } \\
\quad(1,2-\mathrm{DCA})\end{array}$ & Solvent & SC4436 & 32103 & $107-06-2$ & 0.08 & MCL-CA & 0.5 & - \\
\hline $\begin{array}{l}\text { 1,1-Dichloroethene } \\
\quad(1,1-\mathrm{DCE})\end{array}$ & Organic synthesis & SC4436 & 34501 & $75-35-4$ & 0.022 & MCL-CA & 6 & - \\
\hline $\begin{array}{l}\text { trans-1,2-Dichloroethene } \\
\quad(\text { trans-1,2-DCE })\end{array}$ & Solvent & SC4436 & 34546 & $156-60-5$ & 0.018 & MCL-CA & 10 & - \\
\hline Dichlorofluoromethane & Refrigerant and propellant & SC4436 & 77119 & $75-43-4$ & 0.05 & na & na & - \\
\hline $\begin{array}{l}\text { 1,2-Dichloropropane } \\
\quad(1,2-\mathrm{DCP})\end{array}$ & Fumigant & SC4437 & 34541 & $78-87-5$ & 0.004 & MCL-US & 5 & $\mathrm{D}$ \\
\hline $\begin{array}{l}\text { 1,1-Dichloro-2- } \\
\text { propanone }\end{array}$ & Disinfection by-product & SC4437 & 80336 & $513-88-2$ & 0.24 & na & na & - \\
\hline cis-1,3-Dichloropropene & Fumigant & SC4436 & 34704 & $10061-01-5$ & 0.1 & MCL-CA & 0.5 & - \\
\hline $\begin{array}{l}\text { trans-1,3- } \\
\quad \text { Dichloropropene }\end{array}$ & Fumigant & SC4436 & 34699 & $10061-02-6$ & 0.14 & MCL-CA & 0.5 & - \\
\hline $\begin{array}{l}\text { 2,6-Dimethyl-4- } \\
\text { heptanone }\end{array}$ & Solvent, organic synthesis & SC4437 & 77419 & $108-83-8$ & 0.032 & na & na & - \\
\hline $\begin{array}{l}\text { 1,2-Dimethyl- } \\
\text { naphthalene }\end{array}$ & Gasoline hydrocarbon & SC4436 & 68725 & $573-98-8$ & 0.18 & na & na & - \\
\hline $\begin{array}{l}\text { 1,6-Dimethyl- } \\
\text { naphthalene }\end{array}$ & Gasoline hydrocarbon & SC4436 & 68727 & $575-43-9$ & 0.2 & na & na & - \\
\hline 1,4-Dioxane & Solvent stablilizer, solvent & SC4437 & 81582 & $123-91-1$ & 0.7 & NL-CA & 1 & $\mathrm{D}$ \\
\hline 1,3-Dioxolane & Solvent, organic synthesis & SC4437 & 81583 & $646-06-0$ & 0.38 & na & na & - \\
\hline 2-Ethoxyethyl acetate & Solvent, organic synthesis & SC4437 & 68728 & $111-15-9$ & 4.2 & na & na & - \\
\hline Ethyl acetate & Solvent, organic synthesis & SC4437 & 81585 & $141-78-6$ & 0.06 & na & na & - \\
\hline
\end{tabular}


Table 3A. Volatile organic compounds (VOCs), primary uses or sources, comparison benchmarks, and reporting information for the U.S. Geological Survey (USGS) National Water Quality Laboratory (NWOL) Schedules 4436 and 4437. - Continued

[The five-digit USGS parameter code is used to uniquely identify a specific constituent or property. Laboratory reporting level (LRL), interim reporting level (IRL), benchmark type, and benchmark level as of April 30, 2014. Benchmark types: Maximum contaminant level benchmarks are listed as MCL-US when the MCL-US and MCL-CA are identical, and as MCL-CA when the MCL-CA is lower than the MCL-US or no MCL-US exists. HBSL, USGS Health Based Screening Level; MCL-CA, State of California maximum contaminant level; MCL-US, U.S. Environmental Protection Agency (EPA) maximum contaminant level; MCL-HI, Hawaii Department of Health maximum contaminant level; NL-CA, State of California notification level; HAL-US, EPA lifetime health advisory level; RSD5-US, EPA risk specific dose at a risk factor of $10^{-5}$. Abbreviations: CAS, Chemical Abstracts Service; CASRN, Chemical Abstracts Service Registry Number; D, detected in groundwater samples (table 5); na, not available; THM, trihalomethane; $\mu \mathrm{g} / \mathrm{L}$, micrograms per liter; -, not detected]

\begin{tabular}{|c|c|c|c|c|c|c|c|c|}
\hline $\begin{array}{l}\text { Constituent } \\
\text { (synonym or } \\
\text { abbreviation) }\end{array}$ & Primary use or source & $\begin{array}{l}\text { NWOL } \\
\text { schedule }\end{array}$ & $\begin{array}{c}\text { USGS } \\
\text { parameter } \\
\text { code } \\
\end{array}$ & CASRN ${ }^{1}$ & $\begin{array}{l}\text { LRL or } \\
\text { IRL } \\
(\mu \mathrm{g} / \mathrm{L})\end{array}$ & $\begin{array}{c}\text { Benchmark } \\
\text { type }^{2}\end{array}$ & $\begin{array}{c}\text { Benchmark } \\
\text { level } \\
(\mu \mathrm{g} / \mathrm{L})^{2}\end{array}$ & Detection \\
\hline Ethylbenzene & Gasoline hydrocarbon & SC4436 & 34371 & $100-41-4$ & 0.036 & MCL-CA & 300 & - \\
\hline 2-Ethyl-1-hexanol & Solvent & SC4437 & 77311 & $104-76-7$ & 2 & na & na & - \\
\hline $\begin{array}{l}\text { Hexachlorocyclo- } \\
\text { pentadiene }\end{array}$ & Organic synthesis & SC4437 & 34386 & $77-47-4$ & 1.6 & na & na & - \\
\hline Isobutyl acetate & Solvent & SC4437 & 77201 & $110-19-0$ & 0.028 & na & na & - \\
\hline Isophorone & Solvent & SC4437 & 34408 & $78-59-1$ & 5 & na & na & - \\
\hline Isopropyl acetate & Solvent, organic synthesis & SC4437 & 45013 & $108-21-4$ & 0.01 & na & na & - \\
\hline Isopropyl alcohol & Solvent & SC4437 & 77015 & $67-63-0$ & 0.8 & na & na & $-{ }^{6}$ \\
\hline $\begin{array}{l}\text { Methylene chloride } \\
\text { (Dichloromethane) }\end{array}$ & Solvent & SC4436 & 34423 & $75-09-2$ & 0.04 & MCL-US & 5 & - \\
\hline 5-Methyl-2-hexanone & Solvent, organic synthesis & SC4437 & 77179 & $110-12-3$ & 0.022 & na & na & - \\
\hline 4-Methyl-2-pentanol & Solvent & SC4437 & 77113 & $108-11-2$ & 0.2 & na & na & - \\
\hline Naphthalene & Gasoline hydrocarbon & SC4436 & 34696 & $91-20-3$ & 0.18 & $\begin{array}{c}\text { HBSL } \\
(\mathrm{NL}-\mathrm{CA})\end{array}$ & $100(17)$ & - \\
\hline Nitrobenzene & Solvent, organic synthesis & SC4437 & 34447 & $98-95-3$ & 0.6 & na & na & - \\
\hline 2-Nitropropane & Solvent, organic synthesis & SC4437 & 77076 & $79-46-9$ & 0.12 & na & na & - \\
\hline Propyl acetate & Solvent & SC4437 & 45022 & $109-60-4$ & 0.032 & na & na & - \\
\hline$n$-Propylbenzene & Solvent & SC4436 & 77224 & $103-65-1$ & 0.036 & NL-CA & 260 & - \\
\hline Styrene & Gasoline hydrocarbon & SC4436 & 77128 & $100-42-5$ & 0.042 & MCL-US & 100 & - \\
\hline alpha-Terpineol & $\begin{array}{l}\text { Natural, fragrance, } \\
\text { disinfectant }\end{array}$ & SC4437 & 68730 & $98-55-5$ & 6 & na & na & - \\
\hline $\begin{array}{l}\text { 1,1,1,2-Tetrachloro- } \\
\text { ethane }\end{array}$ & Solvent & SC4436 & 77562 & $630-20-6$ & 0.040 & $\begin{array}{c}\text { HBSL } \\
\text { (HAL-US) }\end{array}$ & $70(70)$ & - \\
\hline $\begin{array}{l}\text { Tetrachloroethene } \\
\text { (PCE, } \\
\text { Perchloroethylene) }\end{array}$ & Solvent & SC4436 & 34475 & $127-18-4$ & 0.026 & MCL-US & 5 & $\mathrm{D}$ \\
\hline
\end{tabular}


Table 3A. Volatile organic compounds (VOCs), primary uses or sources, comparison benchmarks, and reporting information for the U.S. Geological Survey (USGS) National Water Quality Laboratory (NWOL) Schedules 4436 and 4437._-Continued

[The five-digit USGS parameter code is used to uniquely identify a specific constituent or property. Laboratory reporting level (LRL), interim reporting level (IRL), benchmark type, and benchmark level as of April 30, 2014. Benchmark types: Maximum contaminant level benchmarks are listed as MCL-US when the MCL-US and MCL-CA are identical, and as MCL-CA when the MCL-CA is lower than the MCL-US or no MCL-US exists. HBSL, USGS Health Based Screening Level; MCL-CA, State of California maximum contaminant level; MCL-US, U.S. Environmental Protection Agency (EPA) maximum contaminant level; MCL-HI, Hawaii Department of Health maximum contaminant level; NL-CA, State of California notification level; HAL-US, EPA lifetime health advisory level; RSD5-US, EPA risk specific dose at a risk factor of $10^{-5}$. Abbreviations: CAS, Chemical Abstracts Service; CASRN, Chemical Abstracts Service Registry Number; D, detected in groundwater samples (table 5); na, not available; THM, trihalomethane; $\mu \mathrm{g} / \mathrm{L}$, micrograms per liter; - , not detected]

\begin{tabular}{|c|c|c|c|c|c|c|c|c|}
\hline $\begin{array}{l}\text { Constituent } \\
\text { (synonym or } \\
\text { abbreviation) }\end{array}$ & Primary use or source & $\begin{array}{l}\text { NWOL } \\
\text { schedule }\end{array}$ & $\begin{array}{l}\text { USGS } \\
\text { parameter } \\
\text { code }\end{array}$ & CASRN $^{1}$ & $\begin{array}{l}\text { LRL or } \\
\text { IRL } \\
(\mu g / L)\end{array}$ & $\begin{array}{c}\text { Benchmark } \\
\text { type }^{2}\end{array}$ & $\begin{array}{c}\text { Benchmark } \\
\text { level } \\
(\mu \mathrm{g} / \mathrm{L})^{2}\end{array}$ & Detection \\
\hline $\begin{array}{l}\text { 1,2,3,4-Tetrahydro- } \\
\text { napthalene (Tetralin) }\end{array}$ & Solvent & SC4436 & 77323 & $119-64-2$ & 0.08 & na & na & - \\
\hline Toluene & Gasoline hydrocarbon & SC4436 & 34010 & $108-88-3$ & 0.02 & MCL-CA & 150 & -6 \\
\hline 1,2,4-Trichlorobenzene & Solvent & SC4436 & 34551 & $120-82-1$ & 0.08 & MCL-CA & 5 & - \\
\hline $\begin{array}{l}\text { 1,1,2-Trichloroethane } \\
\quad(1,1,2 \text {-TCA })\end{array}$ & Solvent & SC4436 & 34511 & $79-00-5$ & 0.046 & MCL-US & 5 & - \\
\hline $\begin{array}{l}\text { Trichloroethene } \\
\text { (TCE, } \\
\text { Trichloroethylene) }\end{array}$ & Solvent & SC4436 & 39180 & 79-01-6 & 0.022 & MCL-US & 5 & $\mathrm{D}$ \\
\hline $\begin{array}{l}\text { Vinyl chloride } \\
\text { (Chloroethene) }\end{array}$ & Organic synthesis & SC4436 & 39175 & $75-01-4$ & 0.06 & MCL-CA & 0.5 & - \\
\hline$m$ - and $p$-Xylene & Gasoline hydrocarbon & SC4436 & 85795 & $\begin{array}{l}179601- \\
23-1\end{array}$ & 0.08 & MCL-CA & ${ }^{8} 1,750$ & - \\
\hline$o$-Xylene & Gasoline hydrocarbon & SC4436 & 77135 & $95-47-6$ & 0.032 & MCL-CA & ${ }^{8} 1,750$ & - \\
\hline
\end{tabular}

${ }^{1}$ This report contains CASRNs ${ }^{\circledR}$, which are registered trademarks of the American Chemical Society. The CAS recommends the verification of the CASRNs through CAS Client Services ${ }^{\mathrm{SM}}$.

${ }^{2}$ Other non-regulatory health-based benchmarks are in parentheses for comparison to HBSL, if available.

${ }^{3}$ The MCL-US benchmark for trihalomethanes is the sum of chloroform, bromoform, bromodichloromethane, and dibromochloromethane.

${ }^{4}$ The median matrix-spike recovery was less than 70 percent. Low recoveries can indicate that the compound was not detected in some samples if it was present at very low concentrations.

${ }^{5}$ The IRL for chlorodifluoromethane changed from $0.038 \mu \mathrm{g} / \mathrm{L}$ to $0.25 \mu \mathrm{g} / \mathrm{L}$ toward the end of the study. The higher value was applied to all data for consistency.

${ }^{6}$ All detections were at concentrations less than the long-term method detection level (LT-MDL; half the LRL), method detection level (MDL), or study reporting level (SRL). Results were not counted as detections.

${ }^{7}$ The NL-CA is not used as the comparison benchmark because it is set at the analytical reporting limit that does not necessarily correspond to a health-based value.

${ }^{8}$ The MCL-CA benchmarks for $m$-plus, $p$-xylene, and $o$-xylene are the sum of all three xylene compounds. 
Table 3B. Pesticides and pesticide degradates, primary uses or sources, comparison benchmarks, and reporting information for the U.S. Geological Survey (USGS) National Water Quality Laboratory (NWOL) Schedule 2437.

[The five-digit USGS parameter code is used to uniquely identify a specific constituent or property. Laboratory reporting level (LRL), interim reporting level (IRL), benchmark type, and benchmark level as of April 30, 2014. Benchmark types: Maximum contaminant level (MCL) benchmarks are listed as MCL-US when the MCL-US and MCL-CA are identical, and as MCL-CA when the MCL-CA is lower than the MCL-US or no MCL-US exists.

HAL-US, U.S. Environmental Protection Agency (EPA) lifetime health advisory level; HBSL, USGS Health Based Screening Level; HHBP, EPA Human Health Benchmarks for Pesticides; MCL-CA, State of California MCL; MCL-US, EPA maximum contaminant level; NL-CA, State of California notification level; RSD5-US, EPA risk specific dose at a risk factor of $10^{-5}$. Abbreviations: CASRN, Chemical Abstract Service Registry Number; D, detected in groundwater samples (tables 6A, B); LT-MDL, long-term method detection level; na, not available; ng/L, nanograms per liter; —, not detected]

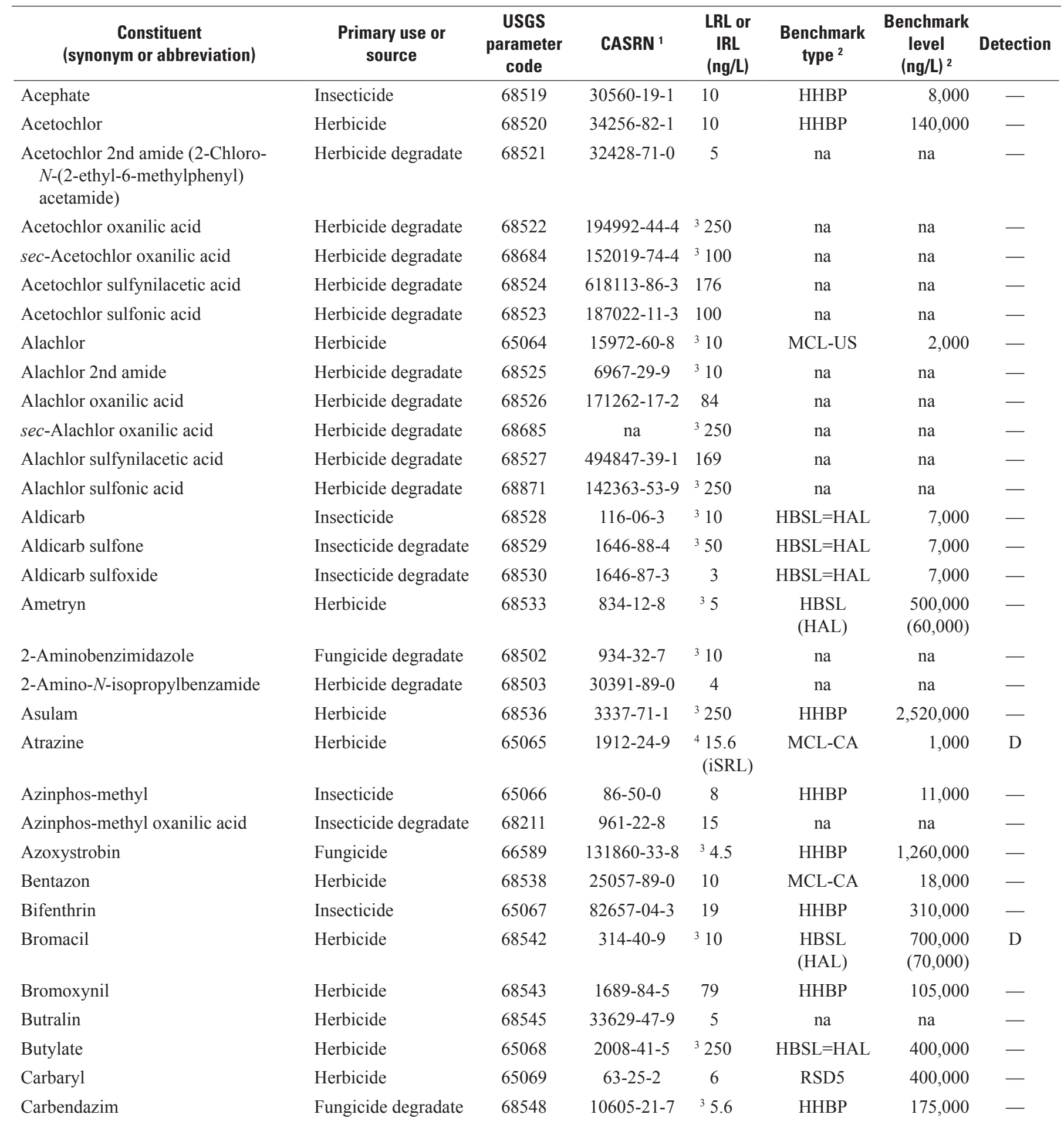


Table 3B. Pesticides and pesticide degradates, primary uses or sources, comparison benchmarks, and reporting information for the U.S. Geological Survey (USGS) National Water Quality Laboratory (NWOL) Schedule 2437.—Continued

[The five-digit USGS parameter code is used to uniquely identify a specific constituent or property. Laboratory reporting level (LRL), interim reporting level (IRL), benchmark type, and benchmark level as of April 30, 2014. Benchmark types: Maximum contaminant level (MCL) benchmarks are listed as MCL-US when the MCL-US and MCL-CA are identical, and as MCL-CA when the MCL-CA is lower than the MCL-US or no MCL-US exists.

HAL-US, U.S. Environmental Protection Agency (EPA) lifetime health advisory level; HBSL, USGS Health Based Screening Level; HHBP, EPA Human Health Benchmarks for Pesticides; MCL-CA, State of California MCL; MCL-US, EPA maximum contaminant level; NL-CA, State of California notification level; RSD5-US, EPA risk specific dose at a risk factor of $10^{-5}$. Abbreviations: CASRN, Chemical Abstract Service Registry Number; D, detected in groundwater samples (tables $6 A, B$ ); LT-MDL, long-term method detection level; na, not available; ng/L, nanograms per liter; —, not detected]

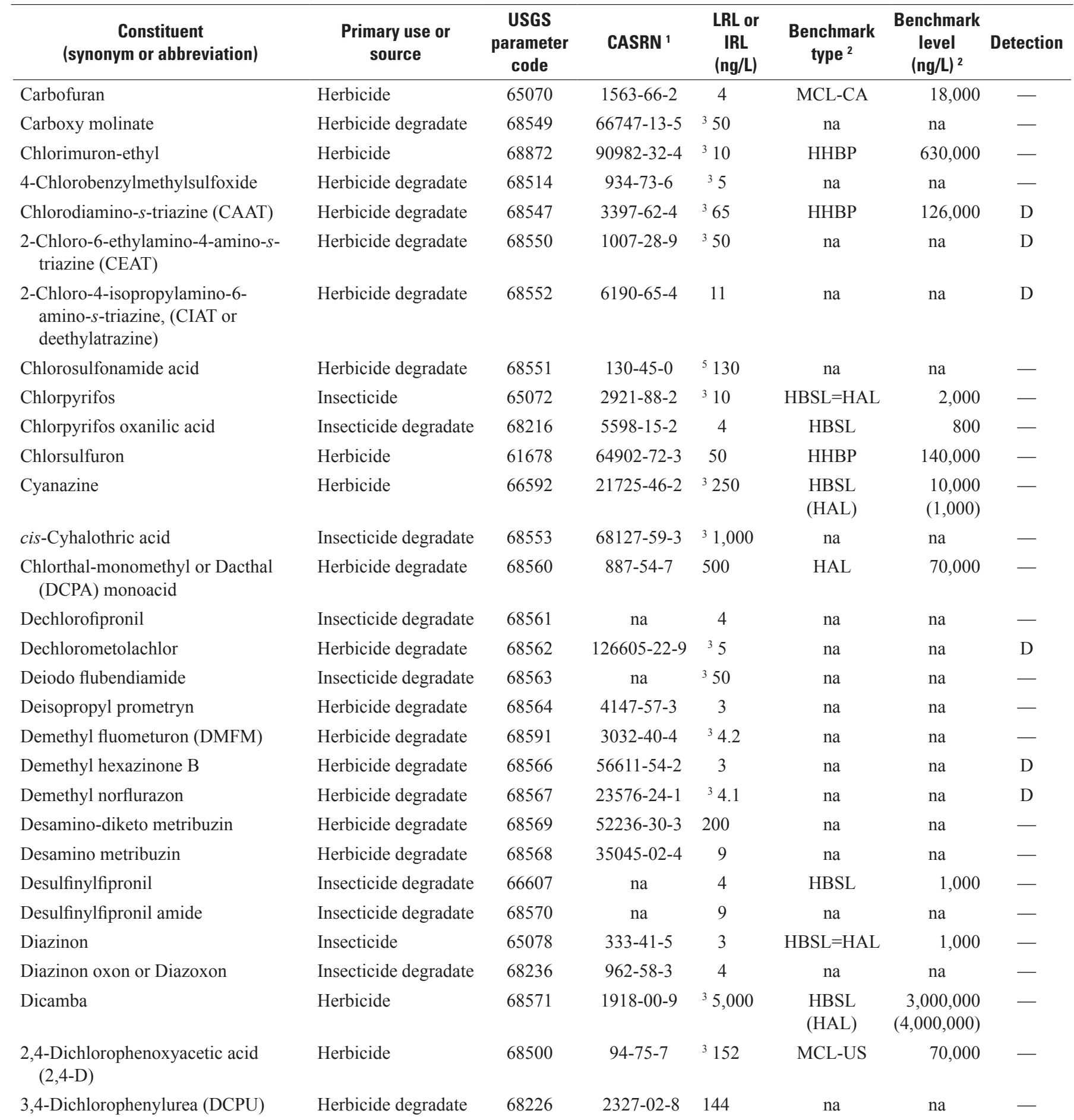


Table 3B. Pesticides and pesticide degradates, primary uses or sources, comparison benchmarks, and reporting information for the U.S. Geological Survey (USGS) National Water Quality Laboratory (NWOL) Schedule 2437.—Continued

[The five-digit USGS parameter code is used to uniquely identify a specific constituent or property. Laboratory reporting level (LRL), interim reporting level (IRL), benchmark type, and benchmark level as of April 30, 2014. Benchmark types: Maximum contaminant level (MCL) benchmarks are listed as MCL-US when the MCL-US and MCL-CA are identical, and as MCL-CA when the MCL-CA is lower than the MCL-US or no MCL-US exists.

HAL-US, U.S. Environmental Protection Agency (EPA) lifetime health advisory level; HBSL, USGS Health Based Screening Level; HHBP, EPA Human Health Benchmarks for Pesticides; MCL-CA, State of California MCL; MCL-US, EPA maximum contaminant level; NL-CA, State of California notification level; RSD5-US, EPA risk specific dose at a risk factor of $10^{-5}$. Abbreviations: CASRN, Chemical Abstract Service Registry Number; D, detected in groundwater samples (tables 6A, B); LT-MDL, long-term method detection level; na, not available; ng/L, nanograms per liter; —, not detected]

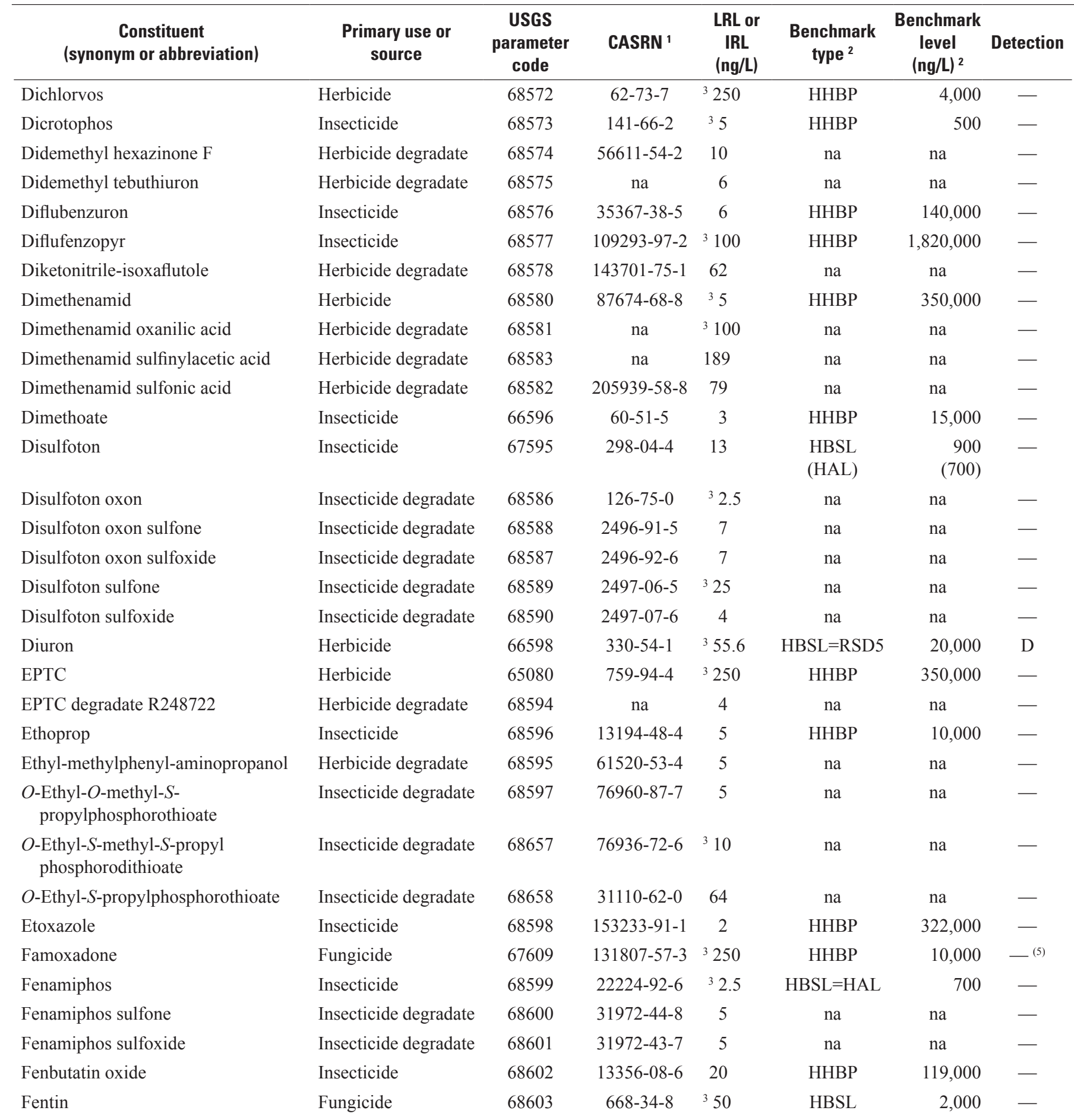


Table 3B. Pesticides and pesticide degradates, primary uses or sources, comparison benchmarks, and reporting information for the U.S. Geological Survey (USGS) National Water Quality Laboratory (NWOL) Schedule 2437.—Continued

[The five-digit USGS parameter code is used to uniquely identify a specific constituent or property. Laboratory reporting level (LRL), interim reporting level (IRL), benchmark type, and benchmark level as of April 30, 2014. Benchmark types: Maximum contaminant level (MCL) benchmarks are listed as MCL-US when the MCL-US and MCL-CA are identical, and as MCL-CA when the MCL-CA is lower than the MCL-US or no MCL-US exists.

HAL-US, U.S. Environmental Protection Agency (EPA) lifetime health advisory level; HBSL, USGS Health Based Screening Level; HHBP, EPA Human Health Benchmarks for Pesticides; MCL-CA, State of California MCL; MCL-US, EPA maximum contaminant level; NL-CA, State of California notification level; RSD5-US, EPA risk specific dose at a risk factor of $10^{-5}$. Abbreviations: CASRN, Chemical Abstract Service Registry Number; D, detected in groundwater samples (tables 6A, B); LT-MDL, long-term method detection level; na, not available; ng/L, nanograms per liter; —, not detected]

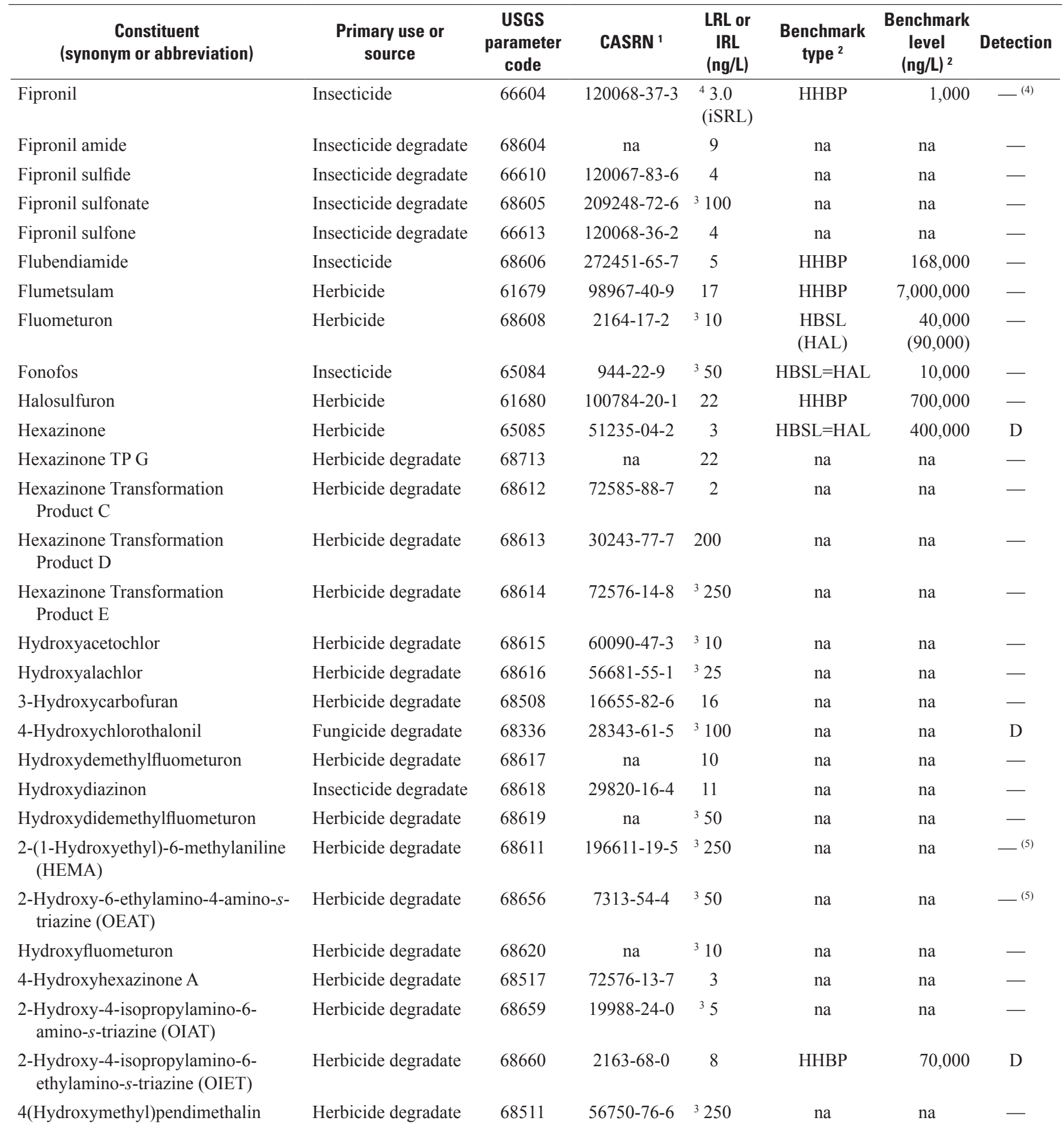


Table 3B. Pesticides and pesticide degradates, primary uses or sources, comparison benchmarks, and reporting information for the U.S. Geological Survey (USGS) National Water Quality Laboratory (NWOL) Schedule 2437.-Continued

[The five-digit USGS parameter code is used to uniquely identify a specific constituent or property. Laboratory reporting level (LRL), interim reporting level (IRL), benchmark type, and benchmark level as of April 30, 2014. Benchmark types: Maximum contaminant level (MCL) benchmarks are listed as MCL-US when the MCL-US and MCL-CA are identical, and as MCL-CA when the MCL-CA is lower than the MCL-US or no MCL-US exists.

HAL-US, U.S. Environmental Protection Agency (EPA) lifetime health advisory level; HBSL, USGS Health Based Screening Level; HHBP, EPA Human Health Benchmarks for Pesticides; MCL-CA, State of California MCL; MCL-US, EPA maximum contaminant level; NL-CA, State of California notification level; RSD5-US, EPA risk specific dose at a risk factor of $10^{-5}$. Abbreviations: CASRN, Chemical Abstract Service Registry Number; D, detected in groundwater samples (tables 6A, B); LT-MDL, long-term method detection level; na, not available; ng/L, nanograms per liter; —, not detected]

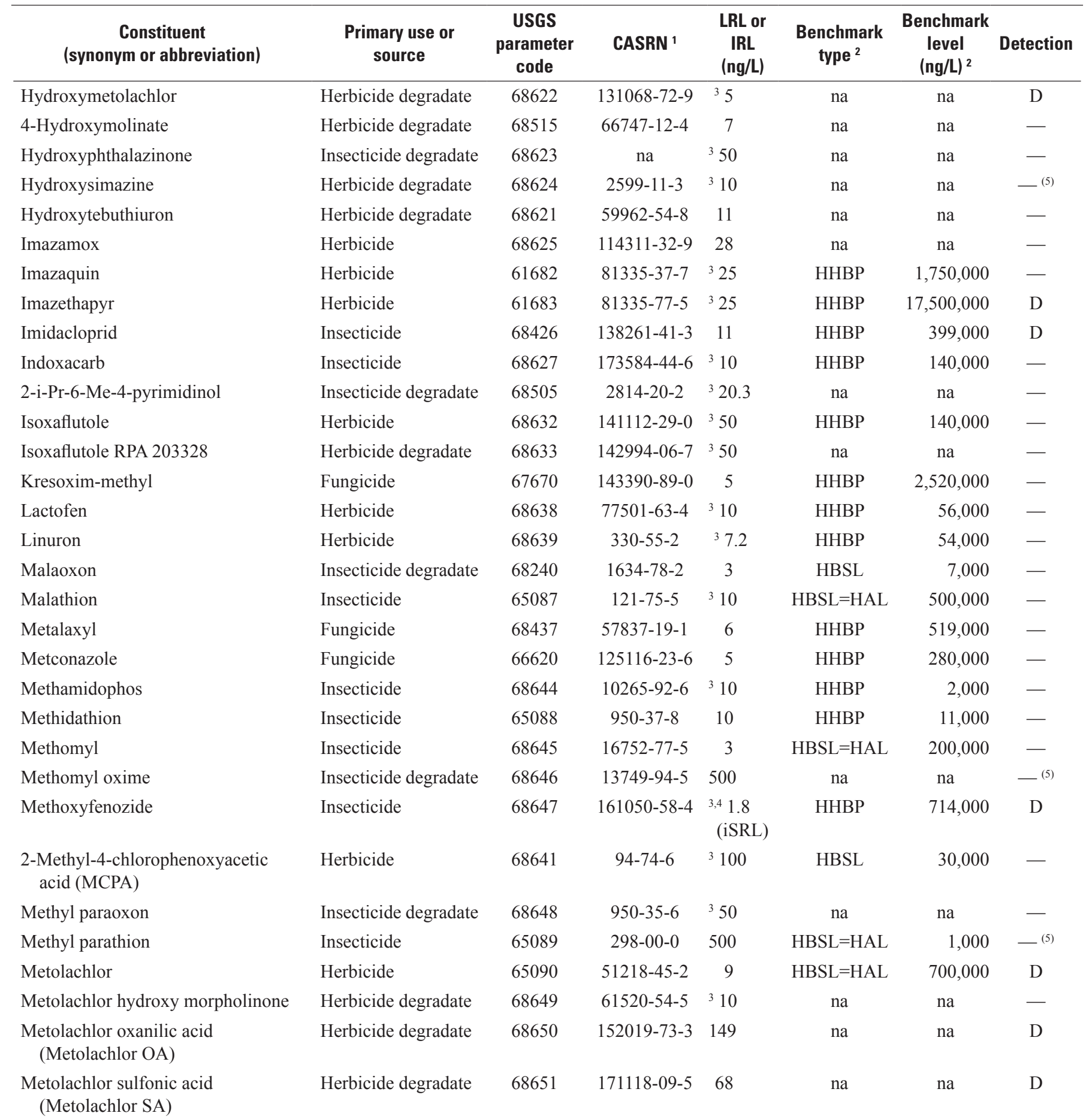


Table 3B. Pesticides and pesticide degradates, primary uses or sources, comparison benchmarks, and reporting information for the U.S. Geological Survey (USGS) National Water Quality Laboratory (NWOL) Schedule 2437.—Continued

[The five-digit USGS parameter code is used to uniquely identify a specific constituent or property. Laboratory reporting level (LRL), interim reporting level (IRL), benchmark type, and benchmark level as of April 30, 2014. Benchmark types: Maximum contaminant level (MCL) benchmarks are listed as MCL-US when the MCL-US and MCL-CA are identical, and as MCL-CA when the MCL-CA is lower than the MCL-US or no MCL-US exists.

HAL-US, U.S. Environmental Protection Agency (EPA) lifetime health advisory level; HBSL, USGS Health Based Screening Level; HHBP, EPA Human Health Benchmarks for Pesticides; MCL-CA, State of California MCL; MCL-US, EPA maximum contaminant level; NL-CA, State of California notification level; RSD5-US, EPA risk specific dose at a risk factor of $10^{-5}$. Abbreviations: CASRN, Chemical Abstract Service Registry Number; D, detected in groundwater samples (tables 6A, B); LT-MDL, long-term method detection level; na, not available; ng/L, nanograms per liter; —, not detected]

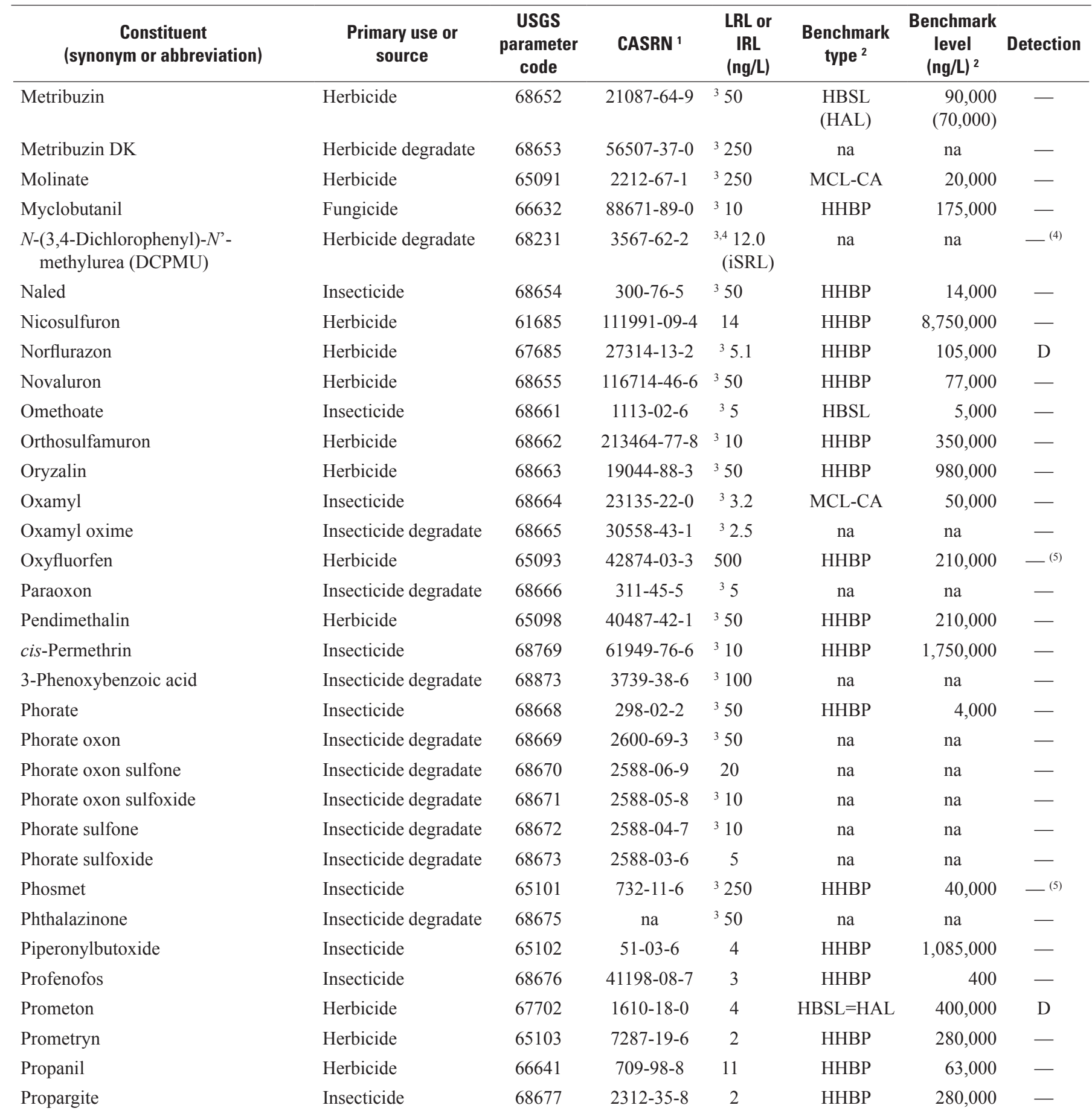


Table 3B. Pesticides and pesticide degradates, primary uses or sources, comparison benchmarks, and reporting information for the U.S. Geological Survey (USGS) National Water Quality Laboratory (NWOL) Schedule 2437.-Continued

[The five-digit USGS parameter code is used to uniquely identify a specific constituent or property. Laboratory reporting level (LRL), interim reporting level (IRL), benchmark type, and benchmark level as of April 30, 2014. Benchmark types: Maximum contaminant level (MCL) benchmarks are listed as MCL-US when the MCL-US and MCL-CA are identical, and as MCL-CA when the MCL-CA is lower than the MCL-US or no MCL-US exists.

HAL-US, U.S. Environmental Protection Agency (EPA) lifetime health advisory level; HBSL, USGS Health Based Screening Level; HHBP, EPA Human Health Benchmarks for Pesticides; MCL-CA, State of California MCL; MCL-US, EPA maximum contaminant level; NL-CA, State of California notification level; RSD5-US, EPA risk specific dose at a risk factor of $10^{-5}$. Abbreviations: CASRN, Chemical Abstract Service Registry Number; D, detected in groundwater samples (tables 6A, B); LT-MDL, long-term method detection level; na, not available; ng/L, nanograms per liter; —, not detected]

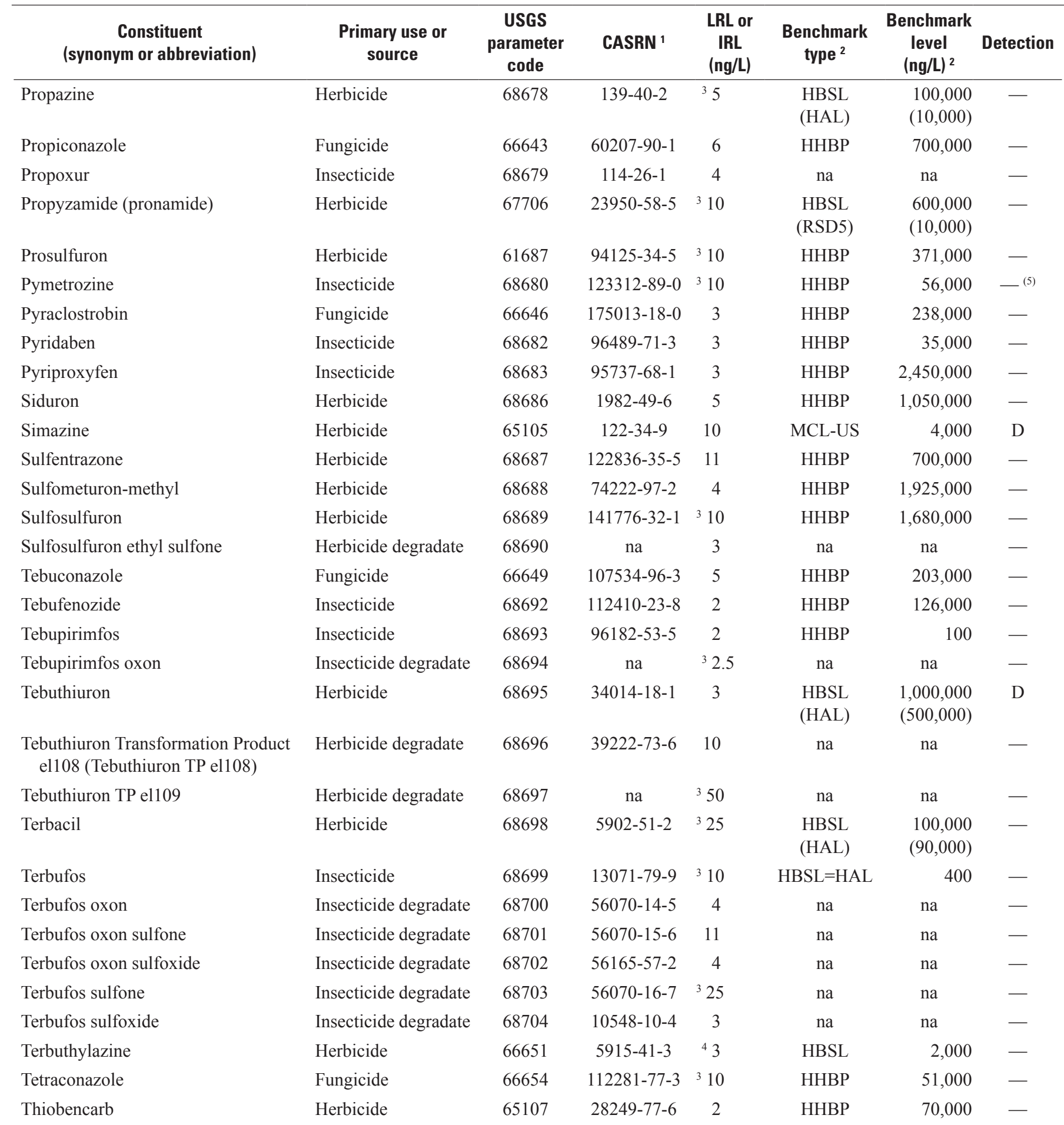


Table 3B. Pesticides and pesticide degradates, primary uses or sources, comparison benchmarks, and reporting information for the U.S. Geological Survey (USGS) National Water Quality Laboratory (NWOL) Schedule 2437.—Continued

[The five-digit USGS parameter code is used to uniquely identify a specific constituent or property. Laboratory reporting level (LRL), interim reporting level (IRL), benchmark type, and benchmark level as of April 30, 2014. Benchmark types: Maximum contaminant level (MCL) benchmarks are listed as MCL-US when the MCL-US and MCL-CA are identical, and as MCL-CA when the MCL-CA is lower than the MCL-US or no MCL-US exists.

HAL-US, U.S. Environmental Protection Agency (EPA) lifetime health advisory level; HBSL, USGS Health Based Screening Level; HHBP, EPA Human Health Benchmarks for Pesticides; MCL-CA, State of California MCL; MCL-US, EPA maximum contaminant level; NL-CA, State of California notification level; RSD5-US, EPA risk specific dose at a risk factor of $10^{-5}$. Abbreviations: CASRN, Chemical Abstract Service Registry Number; D, detected in groundwater samples (tables 6A, B); LT-MDL, long-term method detection level; na, not available; ng/L, nanograms per liter; —, not detected]

\begin{tabular}{|c|c|c|c|c|c|c|c|}
\hline $\begin{array}{c}\text { Constituent } \\
\text { (synonym or abbreviation) }\end{array}$ & $\begin{array}{l}\text { Primary use or } \\
\text { source }\end{array}$ & $\begin{array}{c}\text { USGS } \\
\text { parameter } \\
\text { code }\end{array}$ & CASRN $^{1}$ & $\begin{array}{l}\text { LRL or } \\
\text { IRL } \\
\text { (ng/L) }\end{array}$ & $\begin{array}{c}\text { Benchmark } \\
\text { type }^{2}\end{array}$ & $\begin{array}{c}\text { Benchmark } \\
\text { level } \\
(\mathrm{ng} / \mathrm{L})^{2}\end{array}$ & Detection \\
\hline trans-Permethrin & Insecticide & 68708 & $61949-77-7$ & 4 & HHBP & $1,750,000$ & - \\
\hline Triallate & Herbicide & 68710 & $2303-17-5$ & 16 & НHBP & 175,000 & - \\
\hline 1H-1,2,4-Triazole & Fungicide degradate & 68498 & $288-88-0$ & ${ }^{3} 63.4$ & HHBP & 35,000 & - \\
\hline Tribuphos & Herbicide & 68711 & $78-48-8$ & 2 & НHВР & 7,000 & - \\
\hline Triclopyr & Herbicide & 68712 & $55335-06-3$ & 88 & HHBP & 350,000 & 一 \\
\hline Tridemethyl tebuthiuron & Herbicide degradate & 68714 & na & 76 & na & na & - \\
\hline Trifloxystrobin & Fungicide & 66660 & $141517-21-7$ & 2 & HHBP & 266,000 & - \\
\hline
\end{tabular}

\footnotetext{
${ }^{1}$ This report contains CASRNs ${ }^{\mathbb{R}}$, which is a Registered Trademark of the American Chemical Society. CAS recommends the verification of the CASRNs through CAS Client Services ${ }^{\mathrm{SM}}$.

${ }^{2}$ Other non-regulatory health-based benchmarks are in parantheses for comparison to HBSL if available.

${ }^{3} \mathrm{~A}$ range of LRLs was used. The data are reported with the highest LRL, and detections at concentrations less than the highest LT-MDL (half the highest LRL) are not reported.

${ }^{4}$ Atrazine, fipronil, methoxyfenozide, and DCPMU were assigned interim study reporting levels (iSRLs) based on detections in laboratory prep blanks. Detections in groundwater samples at concentrations less than the iSRLs are not reported and are not counted as detections for the purpose of calculating detection frequencies.

${ }^{5}$ The laboratory did not provide results for all of the groundwater samples for these constituents.
} 
Table 3C. Inorganic constituents, comparative benchmarks, and reporting information.

[The five-digit USGS parameter code is used to uniquely identify a specific constituent or property. The LRL, benchmark type, and benchmark level as of April 30, 2014. Benchmark types: Maximum contaminant level benchmarks are listed as MCL-US when the MCL-US and MCL-CA are identical, and as MCL-CA when the MCL-CA is lower than the MCL-US or no MCL-US exists. AL-US, U.S. Environmental Protection Agency (EPA) action level; HBSL, USGS health-based screening level; MCL-CA, State of California maximum contaminant level; MCL-US, EPA maximum contaminant level; NL-CA, State of California notification level; HAL-US, EPA lifetime health advisory level; SMCL-CA, State of California secondary maximum contaminant level. Abbreviations: CASRN, Chemical Abstracts Service Registry Number; LT-MDL, long-term method detection level; mg/L, milligrams per liter; na, not available; pCi/L, picocuries per liter; pmc, percent modern carbon; $\mathrm{ssL}_{\mathrm{c}}$, sample-specific reporting level; USGS, U.S. Geological Survey; $\mu \mathrm{g} / \mathrm{L}$, micrograms per liter]

\begin{tabular}{|c|c|c|c|c|c|c|}
\hline Constituent & $\begin{array}{c}\text { USGS } \\
\text { parameter } \\
\text { code }\end{array}$ & CASRN & LT-MDL ${ }^{1}$ & Benchmark type ${ }^{2}$ & $\begin{array}{c}\text { Benchmark } \\
\text { level }^{2}\end{array}$ & Units \\
\hline \multicolumn{7}{|c|}{ Trace elements (NWOL Schedule 1948) } \\
\hline Aluminum & 01106 & $7429-90-5$ & 2.2 & MCL-CA & 1,000 & $\mu \mathrm{g} / \mathrm{L}$ \\
\hline Antimony & 01095 & $7440-36-0$ & 0.027 & MCL-US & 6 & $\mu \mathrm{g} / \mathrm{L}$ \\
\hline Arsenic & 01000 & $7440-38-2$ & $0.04,0.10$ & MCL-US & 10 & $\mu \mathrm{g} / \mathrm{L}$ \\
\hline Beryllium & 01010 & $7440-41-7$ & $0.006,0.02$ & MCL-US & 4 & $\mu \mathrm{g} / \mathrm{L}$ \\
\hline Boron & 01020 & $7440-42-8$ & $3,5.0$ & HBSL (NL-CA) & $6,000(1,000)$ & $\mu \mathrm{g} / \mathrm{L}$ \\
\hline Cadmium & 01025 & $7440-43-9$ & $0.016,0.03$ & MCL-US & 5 & $\mu \mathrm{g} / \mathrm{L}$ \\
\hline Chromium & 01030 & $7440-47-3$ & $0.07,0.3$ & MCL-CA & 50 & $\mu \mathrm{g} / \mathrm{L}$ \\
\hline Lead & 01049 & 7439-92-1 & $0.025,0.04$ & AL-US & 15 & $\mu \mathrm{g} / \mathrm{L}$ \\
\hline Lithium & 01130 & $7439-93-2$ & 0.22 & na & na & $\mu \mathrm{g} / \mathrm{L}$ \\
\hline Manganese & 01056 & $7439-96-5$ & $0.15,0.40$ & HBSL (SMCL-CA) & $300(50)$ & $\mu \mathrm{g} / \mathrm{L}$ \\
\hline Molybdenum & 01060 & 7439-98-7 & $0.014,0.05$ & HBSL (HAL-US) & $40(40)$ & $\mu \mathrm{g} / \mathrm{L}$ \\
\hline Nickel & 01065 & $7440-02-0$ & $0.09,0.20$ & MCL-CA & 100 & $\mu \mathrm{g} / \mathrm{L}$ \\
\hline Selenium & 01145 & $7782-49-2$ & $0.03,0.05$ & MCL-US & 50 & $\mu \mathrm{g} / \mathrm{L}$ \\
\hline Silver & 01075 & $7440-22-4$ & $0.005,0.02$ & HBSL (SMCL-CA) & $100(100)$ & $\mu \mathrm{g} / \mathrm{L}$ \\
\hline Strontium & 01080 & $7440-24-6$ & $0.2,0.8$ & HBSL (HAL-US) & $4,000(4,000)$ & $\mu \mathrm{g} / \mathrm{L}$ \\
\hline Thallium ${ }^{4}$ & 01057 & $7440-28-0$ & $0.010,0.03$ & MCL-US & 2 & $\mu \mathrm{g} / \mathrm{L}$ \\
\hline Chloride & 00940 & $16887-00-6$ & $0.06,0.02$ & SMCL-CA & ${ }^{5} 500$ & $\mathrm{mg} / \mathrm{L}$ \\
\hline Fluoride & 00950 & $16984-48-8$ & 0.01 & MCL-CA & 2 & $\mathrm{mg} / \mathrm{L}$ \\
\hline Iodide & 71865 & $7553-56-2$ & 0.001 & na & na & $\mathrm{mg} / \mathrm{L}$ \\
\hline Magnesium & 00925 & 7439-95-4 & 0.011 & na & na & $\mathrm{mg} / \mathrm{L}$ \\
\hline Potassium & 00935 & 7440-09-7 & 0.03 & na & na & $\mathrm{mg} / \mathrm{L}$ \\
\hline Sodium & 00930 & $7440-23-5$ & 0.06 & na & na & $\mathrm{mg} / \mathrm{L}$ \\
\hline Sulfate & 00945 & $14808-79-8$ & $0.09,0.02$ & SMCL-CA & ${ }^{5} 500$ & $\mathrm{mg} / \mathrm{L}$ \\
\hline Silica $\left(\right.$ as $\left.\mathrm{SiO}_{2}\right)$ & 00955 & $7631-86-9$ & 0.018 & na & na & $\mathrm{mg} / \mathrm{L}$ \\
\hline Total dissolved solids (TDS) & 70300 & na & 10 & SMCL-CA & ${ }^{5} 1,000$ & $\mathrm{mg} / \mathrm{L}$ \\
\hline Laboratory alkalinity (as $\left.\mathrm{CaCO}_{3}\right)^{6}$ & 29801 & na & 4.6 & na & na & $\mathrm{mg} / \mathrm{L}$ \\
\hline
\end{tabular}


Table 3C. Inorganic constituents, comparative benchmarks, and reporting information.-Continued

[The five-digit USGS parameter code is used to uniquely identify a specific constituent or property. The LRL, benchmark type, and benchmark level as of April 30, 2014. Benchmark types: Maximum contaminant level benchmarks are listed as MCL-US when the MCL-US and MCL-CA are identical, and as MCL-CA when the MCL-CA is lower than the MCL-US or no MCL-US exists. AL-US, U.S. Environmental Protection Agency (EPA) action level; HBSL, USGS health-based screening level; MCL-CA, State of California maximum contaminant level; MCL-US, EPA maximum contaminant level; NL-CA, State of California notification level; HAL-US, EPA lifetime health advisory level; SMCL-CA, State of California secondary maximum contaminant level. Abbreviations: CASRN, Chemical Abstracts Service Registry Number; LT-MDL, long-term method detection level; mg/L, milligrams per liter; na, not available; $\mathrm{pCi} / \mathrm{L}$, picocuries per liter; pmc, percent modern carbon; $\mathrm{ssL}_{\mathrm{c}}$, sample-specific reporting level; USGS, U.S. Geological Survey; $\mu \mathrm{g} / \mathrm{L}$, micrograms per liter]

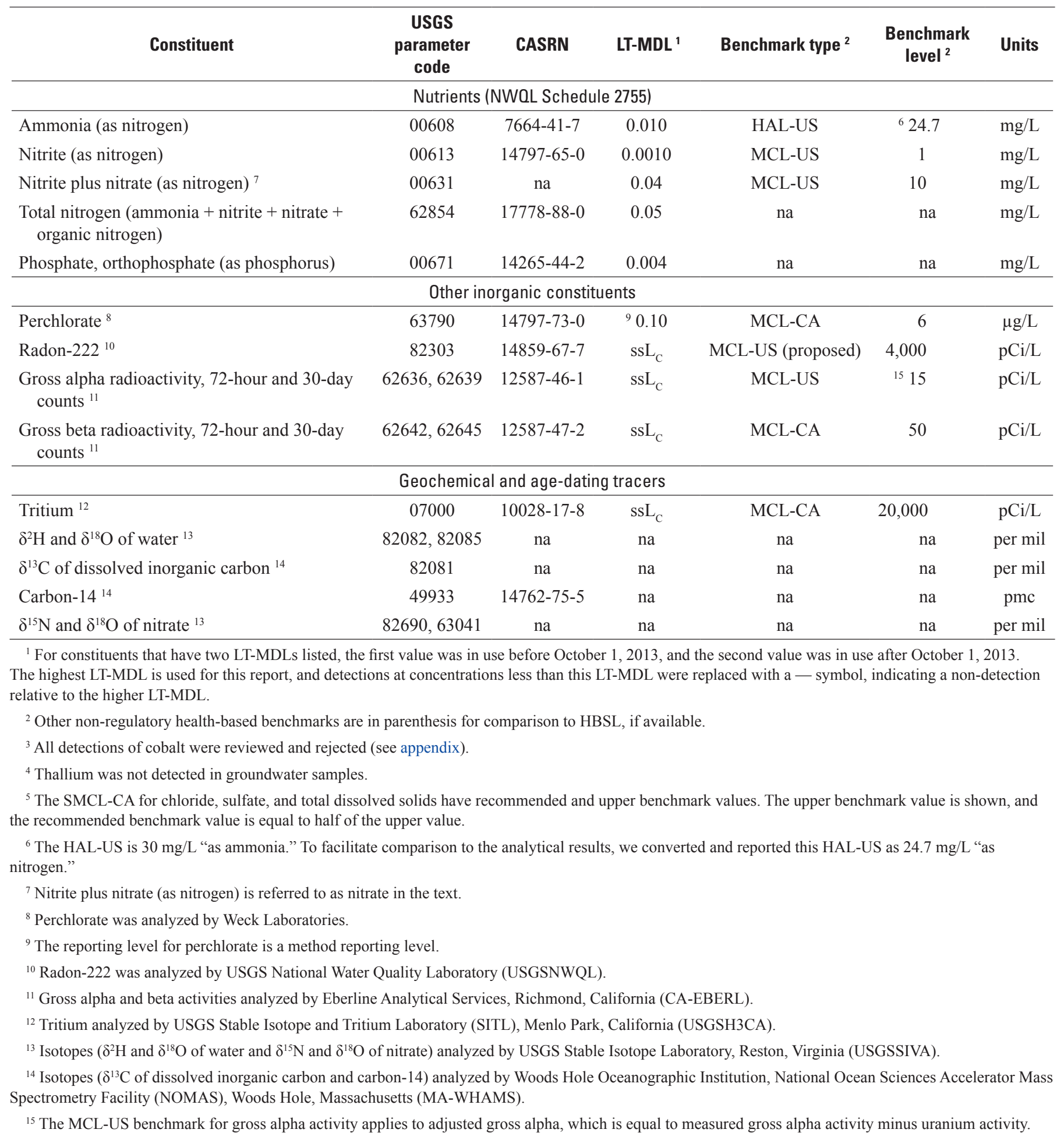


Table 3D. Microbial indicators, comparative benchmarks, and reporting information.

[The five-digit USGS parameter code is used to uniquely identify a specific constituent or property. Method detection limit, benchmark type, and benchmark level as of April 30, 2014. Benchmark types: Maximum contaminant level benchmarks are listed as MCL-US when the MCL-US and MCL-CA are identical, and as MCL-CA when the MCL-CA is lower than the MCL-US or no MCL-US exists. TT-US, U.S. Environmental Protection Agency (EPA) treatment technique - a required process intended to reduce the level of contamination in drinking water; MCL-US, EPA maximum contaminant level. Abbreviations: D, detected in groundwater samples (table 13); MDL, method detection limit; USGS, U.S. Geological Survey]

\begin{tabular}{|c|c|c|c|c|c|c|}
\hline Constituent & $\begin{array}{c}\text { USGS } \\
\text { parameter } \\
\text { code }\end{array}$ & Primary source & MDL & $\begin{array}{c}\text { Benchmark } \\
\text { type }\end{array}$ & Benchmark value & Detection \\
\hline Escherichia coli (E. coli) & 99596 & $\begin{array}{l}\text { Sewage and animal } \\
\text { waste indicator }\end{array}$ & presence/absence & TT-US & 0 & $\mathrm{D}$ \\
\hline \multicolumn{7}{|l|}{ Enterococci } \\
\hline $\begin{array}{l}\text { Total coliform - (including } \\
\text { fecal coliform and E. coli) }\end{array}$ & 99595 & $\begin{array}{l}\text { Sewage and animal } \\
\text { waste indicator }\end{array}$ & presence/absence & MCL-US & $\begin{array}{l}5 \text { percent of samples } \\
\text { positive per month }\end{array}$ & $\mathrm{D}$ \\
\hline
\end{tabular}


Table 4. Water-quality indicators in groundwater samples collected for the Madera/Chowchilla-Kings Shallow Aquifer study unit, Groundwater Ambient Monitoring and Assessment (GAMA) Priority Basin Project, California, August 2013 to April 2014.

[The five-digit USGS parameter code below the constituent name is used to uniquely identify a specific constituent or property. GAMA well identification numbers: S3-MACK-K, Madera/ChowchillaKings Shallow Aquifer Kings study-area grid well; S3-MACK-M, Madera/Chowchilla-Kings Shallow Aquifer Madera/Chowchilla study-area grid well. Benchmark type, benchmark level, and RL as of April 30, 2014. Benchmark types: SMCL-CA, State of California secondary maximum contaminant level; SMCL-US, U.S. Environmental Protection Agency (EPA) secondary maximum contaminant

level. Abbreviations: $\mathrm{CaCO}_{3}$, calcium carbonate; $\mathrm{E}$, estimated or having a higher degree of uncertainty; $\mathrm{mg} / \mathrm{L}$, milligrams per liter; na, not available; nc, not collected; RL, reporting level or range; USGS, U.S. Geological Survey; *, concentration greater than (or outside of range of) the benchmark level; <, less than; >, greater than; ${ }^{\circ} \mathrm{C}$, degrees Celsius; $\mu \mathrm{S} / \mathrm{cm}$, microsiemens per centimeter]

\begin{tabular}{|c|c|c|c|c|c|c|c|c|c|c|c|c|}
\hline $\begin{array}{c}\text { GAMA well } \\
\text { identification } \\
\text { number }\end{array}$ & $\begin{array}{l}\text { Dissolved } \\
\text { oxygen, } \\
\text { field } \\
(\mathrm{mg} / \mathrm{L}) \\
(00300)^{1}\end{array}$ & $\begin{array}{c}\text { Water } \\
\text { temperature, } \\
\text { field } \\
\left({ }^{\circ} \mathrm{C}\right) \\
(00010)\end{array}$ & $\begin{array}{c}\text { pH, field } \\
\text { (standard } \\
\text { units) } \\
(00400)\end{array}$ & $\begin{array}{c}\text { pH, } \\
\text { laboratory } \\
\text { (standard } \\
\text { units) } \\
\text { (00403) }\end{array}$ & $\begin{array}{c}\text { Specific } \\
\text { conductance, } \\
\text { field } \\
\left(\mu \mathrm{S} / \mathrm{cm} \text { at } 25^{\circ} \mathrm{C}\right) \\
(00095)\end{array}$ & $\begin{array}{c}\text { Specific } \\
\text { conductance, } \\
\text { laboratory } \\
\left(\mu \mathrm{S} / \mathrm{cm} \text { at } 25^{\circ} \mathrm{C}\right) \\
(90095)\end{array}$ & $\begin{array}{l}\text { Alkalinity, } \\
\text { field } \\
\text { (mg/L as } \\
\text { CaCO) } \\
(29802)\end{array}$ & $\begin{array}{l}\text { Alkalinity, } \\
\text { laboratory } \\
\text { (mg/L as } \\
\mathrm{CaCO}_{3} \text { ) } \\
(29801)\end{array}$ & $\begin{array}{c}\text { Bicarbonate, } \\
\text { field } \\
(\mathrm{mg} / \mathrm{L}) \\
(\mathbf{6 3 7 8 6})\end{array}$ & $\begin{array}{c}\text { Bicarbonate, } \\
\text { laboratory } \\
(\mathrm{mg} / \mathrm{L})^{3}\end{array}$ & $\begin{array}{c}\text { Carbonate, } \\
\text { field } \\
\text { (mg/L) } \\
(\mathbf{6 3 7 8 8})\end{array}$ & $\begin{array}{c}\text { Carbonate, } \\
\text { laboratory } \\
\text { (mg/L) }^{3}\end{array}$ \\
\hline Benchmark type & na & na & SMCL-US & SMCL-US & SMCL-CA & SMCL-CA & na & na & na & na & na & na \\
\hline Benchmark level & na & na & $<6.5$ or $>8.5$ & $<6.5$ or $>8.5$ & 21,600 & ${ }^{2} 1,600$ & na & na & na & na & na & na \\
\hline [RL] & {$[0.2]$} & {$[0.0-38.5]$} & {$[0-14]$} & {$[0-14]$} & [5] & [5] & [1] & [1] & [1] & [1] & [1] & [1] \\
\hline
\end{tabular}

Madera/Chowchilla-Kings Shallow Aquifer study unit (77 wells sampled)

\begin{tabular}{|c|c|c|c|c|c|c|c|c|c|c|c|c|}
\hline \multicolumn{13}{|c|}{ Madera/Chowchilla study area (28 wells sampled) } \\
\hline S3-MACK-M01 & $<0.2$ & 18.0 & 8.0 & 8.2 & 116 & 112 & nc & 51.7 & nc & 62.1 & $\mathrm{nc}$ & 0.5 \\
\hline S3-MACK-M02 & 3.6 & 21.5 & 7.2 & 7.7 & 295 & 295 & $\mathrm{nc}$ & 80.9 & $\mathrm{nc}$ & 98.2 & nc & 0.2 \\
\hline S3-MACK-M03 & 6.4 & 19.0 & 6.9 & 7.9 & $* 2,110$ & $* 2,080$ & $\mathrm{nc}$ & 516 & nc & 625 & $\mathrm{nc}$ & 2.3 \\
\hline S3-MACK-M04 & 7.7 & 20.5 & 7.1 & 7.6 & 775 & 776 & $\mathrm{nc}$ & 261 & nc & 317 & $\mathrm{nc}$ & 0.6 \\
\hline S3-MACK-M05 & 7.1 & 19.5 & 7.3 & 7.6 & 955 & 940 & $\mathrm{nc}$ & 287 & $\mathrm{nc}$ & 349 & $\mathrm{nc}$ & 0.6 \\
\hline S3-MACK-M06 & 5.7 & 19.0 & 7.4 & 7.7 & 680 & 686 & $\mathrm{nc}$ & 227 & nc & 276 & $\mathrm{nc}$ & 0.6 \\
\hline S3-MACK-M07 & 5.8 & 20.5 & 7.6 & 7.8 & 368 & 370 & 130 & 144 & 157 & 175 & 0.3 & 0.5 \\
\hline S3-MACK-M08 & 11.5 & 19.5 & 7.2 & 7.6 & 763 & 747 & $\mathrm{nc}$ & 211 & nc & 256 & $\mathrm{nc}$ & 0.5 \\
\hline S3-MACK-M09 & 6.8 & 23.0 & 7.3 & 7.6 & 632 & 586 & $\mathrm{nc}$ & 216 & nc & 263 & $\mathrm{nc}$ & 0.5 \\
\hline S3-MACK-M10 & 3.7 & 20.0 & 7.2 & 7.5 & 920 & 937 & $\mathrm{nc}$ & 318 & $\mathrm{nc}$ & 387 & $\mathrm{nc}$ & 0.6 \\
\hline S3-MACK-M11 & 4.7 & 19.5 & 7.3 & 7.5 & 450 & 463 & $\mathrm{nc}$ & 177 & nc & 215 & $\mathrm{nc}$ & 0.3 \\
\hline S3-MACK-M12 & 4.3 & 20.5 & 7.3 & 7.7 & 398 & 378 & $\mathrm{nc}$ & 136 & $\mathrm{nc}$ & 165 & $\mathrm{nc}$ & 0.4 \\
\hline S3-MACK-M13 & 2.1 & 20.0 & 7.5 & 7.7 & 262 & 257 & $\mathrm{nc}$ & 94.2 & $\mathrm{nc}$ & 114 & $\mathrm{nc}$ & 0.3 \\
\hline S3-MACK-M14 & 5.8 & 19.0 & 7.3 & 7.5 & 302 & 290 & $\mathrm{nc}$ & 106 & $\mathrm{nc}$ & 129 & $\mathrm{nc}$ & 0.2 \\
\hline S3-MACK-M15 & 6.2 & 21.0 & 7.4 & 7.7 & 354 & 333 & $\mathrm{nc}$ & 117 & nc & 142 & $\mathrm{nc}$ & 0.3 \\
\hline S3-MACK-M16 & 8.2 & 19.0 & 7.2 & 7.4 & 978 & 965 & $\mathrm{nc}$ & 418 & nc & 509 & $\mathrm{nc}$ & 0.6 \\
\hline S3-MACK-M17 & 8.2 & 19.5 & 7.4 & 8.0 & 1,160 & 1,140 & $\mathrm{nc}$ & 390 & $\mathrm{nc}$ & 471 & $\mathrm{nc}$ & 2.2 \\
\hline S3-MACK-M18 & 7.2 & 22.5 & 7.2 & 7.5 & 372 & 368 & $\mathrm{nc}$ & 109 & $\mathrm{nc}$ & 133 & $\mathrm{nc}$ & 0.2 \\
\hline S3-MACK-M19 & 3.0 & 23.0 & 7.2 & 7.7 & 297 & 294 & $\mathrm{nc}$ & 112 & nc & 136 & $\mathrm{nc}$ & 0.3 \\
\hline S3-MACK-M20 & 4.6 & 21.0 & 7.4 & 7.6 & 221 & 216 & nc & 71.6 & nc & 87 & $\mathrm{nc}$ & 0.2 \\
\hline
\end{tabular}


Table 4. Water-quality indicators in groundwater samples collected for the Madera/Chowchilla-Kings Shallow Aquifer study unit, Groundwater Ambient Monitoring and

Assessment (GAMA) Priority Basin Project, California, August 2013 to April 2014.-Continued

[The five-digit USGS parameter code below the constituent name is used to uniquely identify a specific constituent or property. GAMA well identification numbers: S3-MACK-K, Madera/ChowchillaKings Shallow Aquifer Kings study-area grid well; S3-MACK-M, Madera/Chowchilla-Kings Shallow Aquifer Madera/Chowchilla study-area grid well. Benchmark type, benchmark level, and RL as of April 30, 2014. Benchmark types: SMCL-CA, State of California secondary maximum contaminant level; SMCL-US, U.S. Environmental Protection Agency (EPA) secondary maximum contaminant level. Abbreviations: $\mathrm{CaCO}_{3}$, calcium carbonate; E, estimated or having a higher degree of uncertainty; $\mathrm{mg} / \mathrm{L}$, milligrams per liter; na, not available; nc, not collected; RL, reporting level or range; USGS, U.S. Geological Survey; *, concentration greater than (or outside of range of) the benchmark level; <, less than; >, greater than; ${ }^{\circ} \mathrm{C}$, degrees Celsius; $\mu \mathrm{S} / \mathrm{cm}$, microsiemens per centimeter]

\begin{tabular}{|c|c|c|c|c|c|c|c|c|c|c|c|c|}
\hline $\begin{array}{c}\text { GAMA well } \\
\text { identification } \\
\text { number }\end{array}$ & $\begin{array}{l}\text { Dissolved } \\
\text { oxygen, } \\
\text { field } \\
(\mathrm{mg} / \mathrm{L}) \\
(00300)^{1}\end{array}$ & $\begin{array}{c}\text { Water } \\
\text { temperature, } \\
\text { field } \\
\left({ }^{\circ} \mathrm{C}\right) \\
(00010)\end{array}$ & $\begin{array}{c}\text { pH, field } \\
\text { (standard } \\
\text { units) } \\
(00400)\end{array}$ & $\begin{array}{c}\mathrm{pH}, \\
\text { laboratory } \\
\text { (standard } \\
\text { units) } \\
\text { (00403) }\end{array}$ & $\begin{array}{c}\text { Specific } \\
\text { conductance, } \\
\text { field } \\
\left(\mu \mathrm{S} / \mathrm{cm} \text { at } 25^{\circ} \mathrm{C}\right) \\
(00095)\end{array}$ & $\begin{array}{c}\text { Specific } \\
\text { conductance, } \\
\text { laboratory } \\
\left(\mu \mathrm{S} / \mathrm{cm} \text { at } 25^{\circ} \mathrm{C}\right) \\
(90095)\end{array}$ & $\begin{array}{c}\text { Alkalinity, } \\
\text { field } \\
\text { (mg/L as } \\
\mathrm{CaCO}_{3} \text { ) } \\
(29802)\end{array}$ & $\begin{array}{c}\text { Alkalinity, } \\
\text { laboratory } \\
\text { (mg/L as } \\
\left.\mathrm{CaCO}_{3}\right) \\
(29801)\end{array}$ & $\begin{array}{c}\text { Bicarbonate, } \\
\text { field } \\
\text { (mg/L) } \\
(63786)\end{array}$ & $\begin{array}{l}\text { Bicarbonate, } \\
\text { laboratory } \\
(\mathrm{mg} / \mathrm{L})^{3}\end{array}$ & $\begin{array}{c}\text { Carbonate, } \\
\text { field } \\
\text { (mg/L) } \\
(\mathbf{6 3 7 8 8})\end{array}$ & $\begin{array}{c}\text { Carbonate, } \\
\text { laboratory } \\
(\mathrm{mg} / \mathrm{L})^{3}\end{array}$ \\
\hline Benchmark type & na & na & SMCL-US & SMCL-US & SMCL-CA & SMCL-CA & na & na & na & na & na & na \\
\hline Benchmark level & na & na & $<6.5$ or $>8.5$ & $<6.5$ or $>8.5$ & 21,600 & 21,600 & na & na & na & na & na & na \\
\hline [RL] & {$[0.2]$} & {$[0.0-38.5]$} & [0-14] & {$[0-14]$} & [5] & {$[5]$} & [1] & [1] & [1] & [1] & [1] & [1] \\
\hline \multicolumn{13}{|c|}{ Madera/Chowchilla-Kings Shallow Aquifer study unit (77 wells sampled)—Continued } \\
\hline \multicolumn{13}{|c|}{ Madera/Chowchilla study area (28 wells sampled)—Continued } \\
\hline S3-MACK-M21 & 6.0 & 19.0 & 7.4 & 7.5 & 480 & 436 & 104 & 110 & 127 & 134 & 0.1 & 0.2 \\
\hline S3-MACK-M22 & 5.5 & 21.0 & 7.4 & 7.6 & 355 & 352 & nc & 81.9 & $\mathrm{nc}$ & 99.5 & $\mathrm{nc}$ & 0.2 \\
\hline S3-MACK-M23 & $<0.2$ & 15.5 & 7.6 & 7.7 & 100 & 102 & nc & 51.4 & nc & 62.4 & nc & 0.1 \\
\hline S3-MACK-M24 & 6.3 & 22.0 & 7.0 & 7.3 & 147 & 148 & $\mathrm{nc}$ & 42.7 & nc & 52.0 & $\mathrm{nc}$ & 0.05 \\
\hline S3-MACK-M25 & 6.4 & 24.5 & 7.2 & 7.7 & 256 & 248 & $\mathrm{nc}$ & 88.5 & nc & 107 & $\mathrm{nc}$ & 0.2 \\
\hline S3-MACK-M26 & 6.0 & 22.0 & 7.5 & 7.6 & 295 & 290 & $\mathrm{nc}$ & 70.6 & nc & 85.8 & nc & 0.2 \\
\hline S3-MACK-M27 & 4.1 & 22.5 & 7.2 & 7.4 & 236 & 232 & nc & 91.9 & nc & 112 & nc & 0.1 \\
\hline S3-MACK-M28 & ${ }^{1}<0.2$ & 23.0 & 7.6 & 8.0 & 392 & 391 & 126 & 136 & 152 & 164 & 0.5 & 0.8 \\
\hline \multicolumn{13}{|c|}{ Kings study area (49 wells sampled) } \\
\hline S3-MACK-K01 & 0.5 & 19.5 & 7.1 & 7.5 & $* 2,240$ & $* 2,200$ & nc & 424 & nc & 516 & nc & 0.8 \\
\hline S3-MACK-K02 & 0.4 & 19.5 & 8.4 & 8.3 & $* 1,710$ & $* 1,710$ & nc & 198 & nc & 237 & nc & 2.2 \\
\hline S3-MACK-K03 & $<0.2$ & 26.0 & 7.8 & 8.0 & 547 & 534 & nc & 120 & nc & 145 & nc & 0.7 \\
\hline S3-MACK-K04 & $<0.2$ & 20.0 & 7.5 & 8.0 & 912 & 907 & 302 & nc & 366 & $\mathrm{nc}$ & 1.2 & $\mathrm{nc}$ \\
\hline S3-MACK-K05 & 0.4 & 19.0 & 7.7 & 7.9 & 887 & 865 & $\mathrm{nc}$ & 298 & nc & 361 & nc & 1.3 \\
\hline S3-MACK-K06 & 0.4 & 19.5 & 7.5 & 7.7 & $* 2,180$ & $* 2,140$ & 188 & 236 & 228 & 287 & 0.5 & 0.7 \\
\hline S3-MACK-K07 & $<0.2$ & 19.5 & 7.7 & 8.0 & 1,540 & 1,520 & $\mathrm{nc}$ & 242 & nc & 292 & $\mathrm{nc}$ & 1.3 \\
\hline S3-MACK-K08 & 2.1 & 21.0 & 7.2 & 7.4 & 1,190 & 1,180 & $\mathrm{nc}$ & 359 & nc & 437 & $\mathrm{nc}$ & 0.5 \\
\hline S3-MACK-K09 & ${ }^{1}<0.2$ & 22.0 & 8.2 & 8.2 & 1,030 & 1,010 & $\mathrm{nc}$ & 159 & nc & 191 & $\mathrm{nc}$ & 1.4 \\
\hline S3-MACK-K10 & 8.3 & 24.5 & 7.1 & 7.6 & $* 2,370$ & $* 2,400$ & 313 & $\mathrm{nc}$ & 381 & $\mathrm{nc}$ & 0.3 & $\mathrm{nc}$ \\
\hline
\end{tabular}


Table 4. Water-quality indicators in groundwater samples collected for the Madera/Chowchilla-Kings Shallow Aquifer study unit, Groundwater Ambient Monitoring and Assessment (GAMA) Priority Basin Project, California, August 2013 to April 2014._Continued

[The five-digit USGS parameter code below the constituent name is used to uniquely identify a specific constituent or property. GAMA well identification numbers: S3-MACK-K, Madera/ChowchillaKings Shallow Aquifer Kings study-area grid well; S3-MACK-M, Madera/Chowchilla-Kings Shallow Aquifer Madera/Chowchilla study-area grid well. Benchmark type, benchmark level, and RL as of April 30, 2014. Benchmark types: SMCL-CA, State of California secondary maximum contaminant level; SMCL-US, U.S. Environmental Protection Agency (EPA) secondary maximum contaminant level. Abbreviations: $\mathrm{CaCO}_{3}$, calcium carbonate; $\mathrm{E}$, estimated or having a higher degree of uncertainty; $\mathrm{mg} / \mathrm{L}$, milligrams per liter; na, not available; nc, not collected; RL, reporting level or range; USGS, U.S. Geological Survey; *, concentration greater than (or outside of range of) the benchmark level; <, less than; >, greater than; ${ }^{\circ} \mathrm{C}$, degrees Celsius; $\mu \mathrm{S} / \mathrm{cm}$, microsiemens per centimeter]

\begin{tabular}{|c|c|c|c|c|c|c|c|c|c|c|c|c|}
\hline $\begin{array}{c}\text { GAMA well } \\
\text { identification } \\
\text { number }\end{array}$ & $\begin{array}{c}\text { Dissolved } \\
\text { oxygen, } \\
\text { field } \\
(\mathrm{mg} / \mathrm{L}) \\
(00300)^{1}\end{array}$ & $\begin{array}{c}\text { Water } \\
\text { temperature, } \\
\text { field } \\
\left({ }^{\circ} \mathrm{C}\right) \\
(00010)\end{array}$ & $\begin{array}{l}\text { pH, field } \\
\text { (standard } \\
\text { units) } \\
(00400)\end{array}$ & $\begin{array}{c}\text { pH, } \\
\text { laboratory } \\
\text { (standard } \\
\text { units) } \\
(00403)\end{array}$ & $\begin{array}{c}\text { Specific } \\
\text { conductance, } \\
\text { field } \\
\left(\mu \mathrm{S} / \mathrm{cm} \text { at } 25^{\circ} \mathrm{C}\right) \\
(00095)\end{array}$ & $\begin{array}{c}\text { Specific } \\
\text { conductance, } \\
\text { laboratory } \\
\left(\mu \mathrm{S} / \mathrm{cm} \text { at } 25^{\circ} \mathrm{C}\right) \\
(90095)\end{array}$ & $\begin{array}{l}\text { Alkalinity, } \\
\text { field } \\
\text { (mg/L as } \\
\left.\mathrm{CaCO}_{3}\right) \\
(29802)\end{array}$ & $\begin{array}{c}\text { Alkalinity, } \\
\text { laboratory } \\
\text { (mg/L as } \\
\mathrm{CaCO}_{3} \text { ) } \\
(29801)\end{array}$ & $\begin{array}{c}\text { Bicarbonate, } \\
\text { field } \\
(\mathrm{mg} / \mathrm{L}) \\
(\mathbf{6 3 7 8 6 )}\end{array}$ & $\begin{array}{c}\text { Bicarbonate, } \\
\text { laboratory } \\
(\mathrm{mg} / \mathrm{L})^{3}\end{array}$ & $\begin{array}{c}\text { Carbonate, } \\
\text { field } \\
\text { (mg/L) } \\
(63788)\end{array}$ & $\begin{array}{c}\text { Carbonate, } \\
\text { laboratory } \\
(\mathrm{mg} / \mathrm{L})^{3}\end{array}$ \\
\hline Benchmark type & na & na & SMCL-US & SMCL-US & SMCL-CA & SMCL-CA & na & na & na & na & na & na \\
\hline Benchmark level & na & na & $<6.5$ or $>8.5$ & $<6.5$ or $>8.5$ & 21,600 & 21,600 & na & na & na & na & na & na \\
\hline [RL] & {$[0.2]$} & {$[0.0-38.5]$} & {$[0-14]$} & {$[0-14]$} & {$[5]$} & [5] & [1] & [1] & [1] & [1] & [1] & [1] \\
\hline
\end{tabular}

Madera/Chowchilla-Kings Shallow Aquifer study unit (77 wells sampled)—Continued

Kings study area (49 wells sampled)-Continued

\begin{tabular}{|c|c|c|c|c|c|c|c|c|c|c|c|c|}
\hline S3-MACK-K11 & 4.3 & 23.5 & 7.5 & 7.7 & 938 & 944 & 144 & $\mathrm{nc}$ & 175 & $\mathrm{nc}$ & 0.4 & $\mathrm{nc}$ \\
\hline S3-MACK-K12 & $<0.2$ & 20.5 & 8.4 & 8.3 & 581 & 589 & $\mathrm{nc}$ & 94.5 & $\mathrm{nc}$ & 113 & $\mathrm{nc}$ & 1.0 \\
\hline S3-MACK-K13 & ${ }^{1}<0.2$ & 20.0 & * 8.6 & $* 8.6$ & 1,210 & 1,130 & $\mathrm{nc}$ & 304 & $\mathrm{nc}$ & 357 & $\mathrm{nc}$ & 6.5 \\
\hline S3-MACK-K14 & ${ }^{1}<0.2$ & 20.0 & * 9.1 & $* 9.0$ & 570 & 566 & $\mathrm{nc}$ & 199 & $\mathrm{nc}$ & 221 & nc & 10.2 \\
\hline S3-MACK-K15 & 6.3 & 19.0 & 8.3 & 8.2 & 260 & 248 & $\mathrm{nc}$ & 102 & $\mathrm{nc}$ & 123 & $\mathrm{nc}$ & 0.9 \\
\hline S3-MACK-K16 & 4.5 & 20.0 & 7.0 & 7.4 & 1,330 & 1,330 & $\mathrm{nc}$ & 282 & $\mathrm{nc}$ & 343 & $\mathrm{nc}$ & 0.4 \\
\hline S3-MACK-K17 & 7.1 & 18.0 & 8.5 & 8.3 & 135 & 136 & $\mathrm{nc}$ & 70.5 & $\mathrm{nc}$ & 84.3 & $\mathrm{nc}$ & 0.8 \\
\hline S3-MACK-K18 & 6.7 & 20.0 & 7.1 & 7.6 & 1,210 & 1,210 & 407 & $\mathrm{nc}$ & 496 & $\mathrm{nc}$ & 0.6 & $\mathrm{nc}$ \\
\hline S3-MACK-K19 & 4.3 & 19.5 & 7.3 & 7.5 & 1,160 & 1,160 & $\mathrm{nc}$ & 418 & $\mathrm{nc}$ & 508 & $\mathrm{nc}$ & 0.7 \\
\hline S3-MACK-K20 & 6.7 & 19.5 & 7.2 & 7.5 & 943 & 936 & $\mathrm{nc}$ & 346 & $\mathrm{nc}$ & 421 & $\mathrm{nc}$ & 0.6 \\
\hline S3-MACK-K21 & 0.5 & 19.0 & 7.6 & 7.8 & 547 & 546 & ${ }^{4} 217$ & 228 & ${ }^{4} 264$ & 276 & ${ }^{4} 0.5$ & 0.8 \\
\hline S3-MACK-K22 & 6.3 & 21.5 & 7.4 & 7.7 & 357 & 347 & $\mathrm{nc}$ & 164 & $\mathrm{nc}$ & 199 & $\mathrm{nc}$ & 0.5 \\
\hline S3-MACK-K23 & 6.4 & 23.0 & 7.4 & 7.7 & 182 & 176 & $\mathrm{nc}$ & 76.6 & $\mathrm{nc}$ & 93.0 & $\mathrm{nc}$ & 0.2 \\
\hline S3-MACK-K24 & 5.9 & 20.0 & 7.2 & 7.8 & 795 & 800 & 255 & $\mathrm{nc}$ & 310 & $\mathrm{nc}$ & 0.3 & $\mathrm{nc}$ \\
\hline S3-MACK-K25 & 6.5 & 20.0 & 7.8 & 8.1 & 302 & 296 & $\mathrm{nc}$ & 118 & $\mathrm{nc}$ & 142 & $\mathrm{nc}$ & 0.8 \\
\hline S3-MACK-K26 & 5.8 & 21.5 & 8.0 & 7.9 & 161 & 160 & $\mathrm{nc}$ & 80.9 & $\mathrm{nc}$ & 97.9 & $\mathrm{nc}$ & 0.4 \\
\hline S3-MACK-K27 & 5.2 & 20.0 & 7.3 & 7.8 & 1,110 & 1,100 & $\mathrm{nc}$ & 321 & $\mathrm{nc}$ & 389 & $\mathrm{nc}$ & 1.1 \\
\hline S3-MACK-K28 & 0.7 & 19.0 & 7.7 & 8.0 & 399 & 388 & $\mathrm{nc}$ & 150 & $\mathrm{nc}$ & 90.6 & $\mathrm{nc}$ & 0.4 \\
\hline S3-MACK-K29 & 5.9 & 21.0 & 7.3 & 7.6 & 1,030 & 1,020 & 259 & 256 & 314 & 311 & 0.6 & 0.6 \\
\hline S3-MACK-K30 & 6.3 & 21.0 & 7.6 & 8.0 & 159 & 171 & $\mathrm{nc}$ & 75.0 & nc & 181 & $\mathrm{nc}$ & 0.8 \\
\hline
\end{tabular}


Table 4. Water-quality indicators in groundwater samples collected for the Madera/Chowchilla-Kings Shallow Aquifer study unit, Groundwater Ambient Monitoring and

Assessment (GAMA) Priority Basin Project, California, August 2013 to April 2014.-Continued

[The five-digit USGS parameter code below the constituent name is used to uniquely identify a specific constituent or property. GAMA well identification numbers: S3-MACK-K, Madera/ChowchillaKings Shallow Aquifer Kings study-area grid well; S3-MACK-M, Madera/Chowchilla-Kings Shallow Aquifer Madera/Chowchilla study-area grid well. Benchmark type, benchmark level, and RL as of April 30, 2014. Benchmark types: SMCL-CA, State of California secondary maximum contaminant level; SMCL-US, U.S. Environmental Protection Agency (EPA) secondary maximum contaminant level. Abbreviations: $\mathrm{CaCO}_{3}$, calcium carbonate; E, estimated or having a higher degree of uncertainty; $\mathrm{mg} / \mathrm{L}$, milligrams per liter; na, not available; nc, not collected; RL, reporting level or range; USGS, U.S. Geological Survey; *, concentration greater than (or outside of range of) the benchmark level; <, less than; >, greater than; ${ }^{\circ} \mathrm{C}$, degrees Celsius; $\mu \mathrm{S} / \mathrm{cm}$, microsiemens per centimeter]

\begin{tabular}{|c|c|c|c|c|c|c|c|c|c|c|c|c|}
\hline $\begin{array}{c}\text { GAMA well } \\
\text { identification } \\
\text { number }\end{array}$ & $\begin{array}{l}\text { Dissolved } \\
\text { oxygen, } \\
\text { field } \\
(\mathrm{mg} / \mathrm{L}) \\
(00300)^{1}\end{array}$ & $\begin{array}{c}\text { Water } \\
\text { temperature, } \\
\text { field } \\
\left({ }^{\circ} \mathrm{C}\right) \\
(00010)\end{array}$ & $\begin{array}{c}\text { pH, field } \\
\text { (standard } \\
\text { units) } \\
(00400)\end{array}$ & $\begin{array}{c}\mathrm{pH}, \\
\text { laboratory } \\
\text { (standard } \\
\text { units) } \\
\text { (00403) }\end{array}$ & $\begin{array}{c}\text { Specific } \\
\text { conductance, } \\
\text { field } \\
\left(\mu \mathrm{S} / \mathrm{cm} \text { at } 25^{\circ} \mathrm{C}\right) \\
(00095)\end{array}$ & $\begin{array}{c}\text { Specific } \\
\text { conductance, } \\
\text { laboratory } \\
\left(\mu \mathrm{S} / \mathrm{cm} \text { at } 25^{\circ} \mathrm{C}\right) \\
(90095)\end{array}$ & $\begin{array}{c}\text { Alkalinity, } \\
\text { field } \\
\text { (mg/L as } \\
\mathrm{CaCO}_{3} \text { ) } \\
(29802)\end{array}$ & $\begin{array}{c}\text { Alkalinity, } \\
\text { laboratory } \\
\text { (mg/L as } \\
\left.\mathrm{CaCO}_{3}\right) \\
(29801)\end{array}$ & $\begin{array}{c}\text { Bicarbonate, } \\
\text { field } \\
(\mathrm{mg} / \mathrm{L}) \\
(63786)\end{array}$ & $\begin{array}{c}\text { Bicarbonate, } \\
\text { laboratory } \\
(\mathrm{mg} / \mathrm{L})^{3}\end{array}$ & $\begin{array}{c}\text { Carbonate, } \\
\text { field } \\
\text { (mg/L) } \\
(\mathbf{6 3 7 8 8})\end{array}$ & $\begin{array}{c}\text { Carbonate, } \\
\text { laboratory } \\
\left(^{(\mathrm{mg} / \mathrm{L})^{3}}\right.\end{array}$ \\
\hline Benchmark type & na & na & SMCL-US & SMCL-US & SMCL-CA & SMCL-CA & na & na & na & na & na & na \\
\hline Benchmark level & na & na & $<6.5$ or $>8.5$ & $<6.5$ or $>8.5$ & 21,600 & 21,600 & na & na & na & na & na & na \\
\hline [RL] & [0.2] & {$[0.0-38.5]$} & {$[0-14]$} & {$[0-14]$} & [5] & [5] & [1] & {$[1]$} & [1] & [1] & [1] & [1] \\
\hline \multicolumn{13}{|c|}{ Madera/Chowchilla-Kings Shallow Aquifer study unit (77 wells sampled)_Continued } \\
\hline \multicolumn{13}{|c|}{ Kings study area (49 wells sampled)_Continued } \\
\hline S3-MACK-K31 & 4.4 & 21.0 & 7.9 & 8.1 & 216 & 215 & nc & 81.9 & $\mathrm{nc}$ & 98.7 & nc & 0.6 \\
\hline S3-MACK-K32 & 7.6 & 19.5 & 7.6 & 7.9 & 139 & 137 & $\mathrm{nc}$ & 55.7 & $\mathrm{nc}$ & 67.4 & $\mathrm{nc}$ & 0.2 \\
\hline S3-MACK-K33 & 5.5 & 19.0 & 7.1 & 7.7 & 779 & 780 & 229 & $\mathrm{nc}$ & 279 & $\mathrm{nc}$ & 0.2 & $\mathrm{nc}$ \\
\hline S3-MACK-K34 & 6.3 & 23.0 & 6.8 & 7.4 & 686 & 693 & 268 & $\mathrm{nc}$ & 326 & $\mathrm{nc}$ & 0.2 & $\mathrm{nc}$ \\
\hline S3-MACK-K35 & 6.3 & 22.0 & 7.5 & 7.8 & 210 & 207 & $\mathrm{nc}$ & 81.3 & $\mathrm{nc}$ & 98.6 & $\mathrm{nc}$ & 0.3 \\
\hline S3-MACK-K36 & 7.0 & 22.5 & 7.3 & 7.5 & 355 & 351 & $\mathrm{nc}$ & 145 & $\mathrm{nc}$ & 176 & $\mathrm{nc}$ & 0.3 \\
\hline S3-MACK-K37 & 3.6 & 22.0 & 7.5 & 7.8 & 204 & 203 & $\mathrm{nc}$ & 96.5 & $\mathrm{nc}$ & 117 & $\mathrm{nc}$ & 0.3 \\
\hline S3-MACK-K38 & 5.2 & 21.0 & $* 6.4$ & 6.8 & 784 & 809 & 133 & $\mathrm{nc}$ & 162 & $\mathrm{nc}$ & 0.02 & $\mathrm{nc}$ \\
\hline S3-MACK-K39 & 6.3 & 19.5 & 7.6 & 7.9 & 671 & 631 & $\mathrm{nc}$ & 224 & $\mathrm{nc}$ & 271 & $\mathrm{nc}$ & 1.0 \\
\hline S3-MACK-K40 & E 5.6 & 20.0 & 7.0 & 8.0 & 1,010 & 985 & $\mathrm{nc}$ & 324 & $\mathrm{nc}$ & 392 & $\mathrm{nc}$ & 1.8 \\
\hline S3-MACK-K41 & 5.7 & 22.0 & 7.4 & 8.0 & 890 & 864 & $\mathrm{nc}$ & 294 & $\mathrm{nc}$ & 355 & $\mathrm{nc}$ & 1.6 \\
\hline S3-MACK-K42 & 7.1 & 22.0 & 7.6 & 7.9 & 866 & 852 & $\mathrm{nc}$ & 263 & $\mathrm{nc}$ & 318 & $\mathrm{nc}$ & 1.2 \\
\hline S3-MACK-K43 & 6.7 & 19.5 & 7.2 & 7.6 & 944 & 935 & 328 & 345 & 399 & 419 & 0.5 & 0.8 \\
\hline S3-MACK-K44 & 3.7 & 21.0 & 6.9 & 7.5 & 1,130 & 1,100 & nc & 280 & $\mathrm{nc}$ & 341 & $\mathrm{nc}$ & 0.5 \\
\hline S3-MACK-K45 & 6.3 & 20.0 & 7.6 & 7.8 & 631 & 619 & $\mathrm{nc}$ & 253 & $\mathrm{nc}$ & 307 & $\mathrm{nc}$ & 0.9 \\
\hline S3-MACK-K46 & $<0.2$ & 18.0 & 6.6 & 7.1 & 445 & 428 & 228 & 240 & 278 & 292 & 0.1 & 0.2 \\
\hline S3-MACK-K47 & 5.9 & 20.0 & 7.1 & 7.5 & 1,120 & 1,100 & $\mathrm{nc}$ & 240 & $\mathrm{nc}$ & 292 & $\mathrm{nc}$ & 0.4 \\
\hline S3-MACK-K48 & 3.7 & 20.5 & 7.2 & 7.7 & 551 & 535 & $\mathrm{nc}$ & 210 & $\mathrm{nc}$ & 255 & $\mathrm{nc}$ & 0.6 \\
\hline
\end{tabular}


Table 4. Water-quality indicators in groundwater samples collected for the Madera/Chowchilla-Kings Shallow Aquifer study unit, Groundwater Ambient Monitoring and Assessment (GAMA) Priority Basin Project, California, August 2013 to April 2014._Continued

[The five-digit USGS parameter code below the constituent name is used to uniquely identify a specific constituent or property. GAMA well identification numbers: S3-MACK-K, Madera/ChowchillaKings Shallow Aquifer Kings study-area grid well; S3-MACK-M, Madera/Chowchilla-Kings Shallow Aquifer Madera/Chowchilla study-area grid well. Benchmark type, benchmark level, and RL as of April 30, 2014. Benchmark types: SMCL-CA, State of California secondary maximum contaminant level; SMCL-US, U.S. Environmental Protection Agency (EPA) secondary maximum contaminant level. Abbreviations: $\mathrm{CaCO}_{3}$, calcium carbonate; $\mathrm{E}$, estimated or having a higher degree of uncertainty; $\mathrm{mg} / \mathrm{L}$, milligrams per liter; na, not available; nc, not collected; RL, reporting level or range; USGS, U.S. Geological Survey; *, concentration greater than (or outside of range of) the benchmark level; <, less than; >, greater than; ${ }^{\circ} \mathrm{C}$, degrees Celsius; $\mu \mathrm{S} / \mathrm{cm}$, microsiemens per centimeter]

\begin{tabular}{|c|c|c|c|c|c|c|c|c|c|c|c|c|}
\hline $\begin{array}{c}\text { GAMA well } \\
\text { identification } \\
\text { number }\end{array}$ & $\begin{array}{c}\text { Dissolved } \\
\text { oxygen, } \\
\text { field } \\
(\mathrm{mg} / \mathrm{L}) \\
(00300)^{1}\end{array}$ & $\begin{array}{c}\text { Water } \\
\text { temperature, } \\
\text { field } \\
\left({ }^{\circ} \mathrm{C}\right) \\
(00010)\end{array}$ & $\begin{array}{c}\text { pH, field } \\
\text { (standard } \\
\text { units) } \\
(00400)\end{array}$ & $\begin{array}{c}\mathrm{pH}, \\
\text { laboratory } \\
\text { (standard } \\
\text { units) } \\
\text { (00403) }\end{array}$ & $\begin{array}{c}\text { Specific } \\
\text { conductance, } \\
\text { field } \\
\left(\mu \mathrm{S} / \mathrm{cm} \text { at } 25^{\circ} \mathrm{C}\right) \\
(00095)\end{array}$ & $\begin{array}{c}\text { Specific } \\
\text { conductance, } \\
\text { laboratory } \\
\left(\mu \mathrm{S} / \mathrm{cm} \text { at } 25^{\circ} \mathrm{C}\right) \\
(90095)\end{array}$ & $\begin{array}{l}\text { Alkalinity, } \\
\text { field } \\
\text { (mg/L as } \\
\mathrm{CaCO}_{3} \text { ) } \\
(29802)\end{array}$ & $\begin{array}{c}\text { Alkalinity, } \\
\text { laboratory } \\
\text { (mg/L as } \\
\left.\mathrm{CaCO}_{3}\right) \\
(29801)\end{array}$ & $\begin{array}{c}\text { Bicarbonate, } \\
\text { field } \\
(\mathrm{mg} / \mathrm{L}) \\
(63786)\end{array}$ & $\begin{array}{c}\text { Bicarbonate, } \\
\text { laboratory } \\
(\mathrm{mg} / \mathrm{L})^{3}\end{array}$ & $\begin{array}{c}\text { Carbonate, } \\
\text { field } \\
(\mathrm{mg} / \mathrm{L}) \\
(\mathbf{6 3 7 8 8})\end{array}$ & $\begin{array}{c}\text { Carbonate, } \\
\text { laboratory } \\
(\mathrm{mg} / \mathrm{L})^{3}\end{array}$ \\
\hline Benchmark type & na & na & SMCL-US & SMCL-US & SMCL-CA & SMCL-CA & na & na & na & na & na & na \\
\hline Benchmark level & na & na & $<6.5$ or $>8.5$ & $<6.5$ or $>8.5$ & ${ }^{2} 1,600$ & 21,600 & na & na & na & na & na & na \\
\hline [RL] & {$[0.2]$} & {$[0.0-38.5]$} & {$[0-14]$} & {$[0-14]$} & [5] & [5] & [1] & [1] & [1] & [1] & [1] & [1] \\
\hline \multicolumn{13}{|c|}{ Madera/Chowchilla-Kings Shallow Aquifer study unit (77 wells sampled)_Continued } \\
\hline \multicolumn{13}{|c|}{ Kings study area ( 49 wells sampled) —Continued } \\
\hline S3-MACK-K49 & 5.2 & 21.5 & 7.2 & 7.5 & 717 & 709 & $\mathrm{nc}$ & 274 & $\mathrm{nc}$ & 333 & $\mathrm{nc}$ & 0.5 \\
\hline
\end{tabular}

${ }^{1}$ Hydrogen-sulfide smell noted during sampling.

${ }^{2}$ The SMCL-CA for specific conductance has recommended and upper benchmark values. The upper value is used in this report.

${ }^{3}$ Bicarbonate and carbonate concentrations were calculated from the laboratory alkalinity and $\mathrm{pH}$ values by using the advanced speciation method (http://or.water.usgs.gov/alk/methods.html) with $\mathrm{pK} \mathrm{K}_{1}=6.35$, $\mathrm{pK}_{2}=10.33$, and $\mathrm{pK}_{\mathrm{w}}=14$.

${ }^{4}$ Alkalinity was determined in the field by using the incremental titration method (parameter code 39086) at S3-MACK-K21; bicarbonate (00453) and carbonate (00452) were calculated from the field value; all other field values were measured by using the Gran titration method. 
Table 5. Volatile organic compounds (VOCs) detected in groundwater samples collected for the Madera/Chowchilla-Kings Shallow Aquifer study unit, Groundwater Ambient Monitoring and Assessment (GAMA) Priority Basin Project, California, August 2013 to April 2014.

[The five-digit USGS parameter code below the constituent name is used to uniquely identify a specific constituent or property. Samples from all 77 grid wells were analyzed, but only samples with detections are listed unless otherwise noted. Detected constituents are grouped by primary use or source and listed in order of decreasing detection frequency in the 77 grid wells. All constituents are listed in table $3 A$. GAMA well identification numbers: S3-MACK-K, Madera/Chowchilla-Kings Shallow Aquifer Kings study-area grid well; S3-MACK-M, Madera/Chowchilla-Kings Shallow Aquifer Madera/ Chowchilla study-area grid well. Benchmark types: Maximum contaminant level benchmarks are listed as MCL-US when the MCL-US and MCL-CA are identical, and as MCL-CA when the MCL-CA is lower than the MCL-US or no MCL-US exists. HBSL, USGS health-based screening level; MCL-CA; State of California maximum contaminant level; MCL-HI, State of Hawaii maximum contaminant level; MCL-US, U.S. Environmental Protection Agency (EPA) maximum contaminant level; NL-CA, State of California notification level. Benchmark type, benchmark level, and LRL as of April 30, 2014. Abbreviations: LRL, laboratory reporting level; na, not available; SRL, study reporting level; USGS, U.S. Geological Survey; $\mu \mathrm{g} / \mathrm{L}$, micrograms per liter; *, concentration greater than the benchmark level; -, not detected]

\begin{tabular}{|c|c|c|c|c|c|c|c|c|c|c|c|}
\hline \multirow{2}{*}{$\begin{array}{c}\text { Primary use or } \\
\text { source }\end{array}$} & \multicolumn{4}{|c|}{ Fumigants } & \multicolumn{2}{|c|}{ Gasoline oxygenate } & \multicolumn{2}{|c|}{ Trihalomethanes } & \multicolumn{3}{|c|}{ Gasoline hydrocarbons } \\
\hline & $\begin{array}{c}\text { 1,2-Dichloro- } \\
\text { propane } \\
\text { (1,2-DCP) } \\
(\mu \mathrm{g} / \mathrm{L}) \\
(34541)\end{array}$ & $\begin{array}{c}\text { 1,2,3-Trichloro- } \\
\text { propane } \\
(1,2,3-T C P) \\
(\mu \mathrm{g} / \mathrm{L}) \\
(77443) \\
\end{array}$ & $\begin{array}{c}\text { 1,2-Dibromo- } \\
\text { 3-chloropropane } \\
\text { (DBCP) } \\
(\mu \mathrm{g} / \mathrm{L}) \\
(82625)\end{array}$ & $\begin{array}{c}\text { 1,2-Dibro- } \\
\text { moethane } \\
\text { (EDB) } \\
(\mu \mathrm{g} / \mathrm{L}) \\
(77651)\end{array}$ & $\begin{array}{c}\text { Methyl tert- } \\
\text { butyl ether } \\
\text { (MTBE) } \\
(\mu \mathrm{g} / \mathrm{L}) \\
(78032)\end{array}$ & $\begin{array}{c}\text { tert-butyl alcohol } \\
\text { (TBA; 2-propanol) } \\
(\mu \mathrm{g} / \mathrm{L}) \\
(77035)\end{array}$ & $\begin{array}{c}\text { Chloroform } \\
\text { (trichloro- } \\
\text { methane) } \\
(\mu \mathrm{g} / \mathrm{L}) \\
(32106)\end{array}$ & $\begin{array}{c}\text { Bromodi- } \\
\text { chloro- } \\
\text { methane } \\
(\mu \mathrm{g} / \mathrm{L}) \\
(32101)\end{array}$ & $\begin{array}{c}\text { Butane } \\
(\mu g / L) \\
(81563)\end{array}$ & $\begin{array}{c}\text { Benzene } \\
(\mu \mathrm{g} / \mathrm{L}) \\
(34030)\end{array}$ & $\begin{array}{c}n \text {-Pentane } \\
(\mu \mathrm{g} / \mathrm{L}) \\
(81604)\end{array}$ \\
\hline Benchmark type & MCL-US & HBSL & MCL-US & MCL-US & MCL-CA & NL-CA & MCL-US & MCL-US & na & MCL-CA & na \\
\hline $\begin{array}{l}\text { Benchmark level } \\
\text { ( } \mu \mathrm{g} / \mathrm{L})\end{array}$ & 5 & 30 & 0.2 & 0.05 & 13 & 12 & 180 & ${ }^{1} 80$ & na & 1 & na \\
\hline$[\mathrm{LRL}]^{2}(\mu \mathrm{g} / \mathrm{L})$ & {$[0.004]$} & {$[0.006]$} & {$[0.02]$} & {$[0.004]$} & {$[0.012]$} & {$[0.24]$} & {$[0.03]$} & {$[0.034]$} & {$[0.038]$} & {$[0.026]$} & {$[0.022]$} \\
\hline \multicolumn{12}{|c|}{ Madera/Chowchilla-Kings Shallow Aquifer study unit (77 wells sampled) } \\
\hline $\begin{array}{l}\text { Number of wells } \\
\text { with detections }\end{array}$ & 17 & 16 & 11 & 3 & 9 & 1 & 7 & 2 & 3 & 1 & 1 \\
\hline $\begin{array}{l}\text { Detection frequency } \\
\text { (percent) }\end{array}$ & 22 & 21 & 14 & 4 & 12 & 1 & 9 & 3 & 4 & 1 & 1 \\
\hline $\begin{array}{l}\text { Total detections } \\
\text { (number) }\end{array}$ & - & - & - & - & - & - & - & - & - & - & - \\
\hline \multicolumn{12}{|c|}{ Madera/Chowchilla study area (28 wells sampled) } \\
\hline S3-MACK-M08 & 0.011 & 0.146 & 0.18 & - & - & - & - & - & - & - & - \\
\hline S3-MACK-M09 & 0.018 & 0.033 & - & - & - & 0.18 & - & - & - & - & - \\
\hline S3-MACK-M10 & - & - & - & - & - & - & 0.030 & - & - & - & - \\
\hline S3-MACK-M11 & - & 0.007 & 0.13 & - & - & - & - & - & - & - & - \\
\hline S3-MACK-M12 & - & - & $* 0.23$ & - & - & - & - & - & - & - & - \\
\hline S3-MACK-M14 & 0.835 & 0.326 & $* 0.32$ & - & - & - & - & - & - & - & - \\
\hline S3-MACK-M15 & 0.524 & 0.128 & - & 0.008 & - & - & - & - & - & - & - \\
\hline S3-MACK-M18 & - & - & 0.01 & - & - & - & - & - & - & - & - \\
\hline S3-MACK-M19 & - & - & $* 0.39$ & - & - & - & - & - & - & - & - \\
\hline S3-MACK-M22 & & 0.006 & 0.01 & - & - & - & - & - & - & - & - \\
\hline
\end{tabular}


Table 5. Volatile organic compounds (VOCs) detected in groundwater samples collected for the Madera/Chowchilla-Kings Shallow Aquifer study unit, Groundwater Ambient Monitoring and Assessment (GAMA) Priority Basin Project, California, August 2013 to April 2014._Continued

[The five-digit USGS parameter code below the constituent name is used to uniquely identify a specific constituent or property. Samples from all 77 grid wells were analyzed, but only samples with detections are listed unless otherwise noted. Detected constituents are grouped by primary use or source and listed in order of decreasing detection frequency in the 77 grid wells. All constituents are listed in table 3A. GAMA well identification numbers: S3-MACK-K, Madera/Chowchilla-Kings Shallow Aquifer Kings study-area grid well; S3-MACK-M, Madera/Chowchilla-Kings Shallow Aquifer Madera/ Chowchilla study-area grid well. Benchmark types: Maximum contaminant level benchmarks are listed as MCL-US when the MCL-US and MCL-CA are identical, and as MCL-CA when the MCL-CA is lower than the MCL-US or no MCL-US exists. HBSL, USGS health-based screening level; MCL-CA; State of California maximum contaminant level; MCL-HI, State of Hawaii maximum contaminant level; MCL-US, U.S. Environmental Protection Agency (EPA) maximum contaminant level; NL-CA, State of California notification level. Benchmark type, benchmark level, and LRL as of April 30, 2014. Abbreviations: LRL, laboratory reporting level; na, not available; SRL, study reporting level; USGS, U.S. Geological Survey; $\mu \mathrm{g} / \mathrm{L}$, micrograms per liter; *, concentration greater than the benchmark level;

- not detected]

\begin{tabular}{|c|c|c|c|c|c|c|c|c|c|c|c|}
\hline \multirow{2}{*}{$\begin{array}{c}\text { Primary use or } \\
\text { source }\end{array}$} & \multicolumn{4}{|c|}{ Fumigants } & \multicolumn{2}{|c|}{ Gasoline oxygenate } & \multicolumn{2}{|c|}{ Trihalomethanes } & \multicolumn{3}{|c|}{ Gasoline hydrocarbons } \\
\hline & $\begin{array}{c}\text { 1,2-Dichloro- } \\
\text { propane } \\
\text { (1,2-DCP) } \\
(\mu \mathrm{g} / \mathrm{L}) \\
(34541)\end{array}$ & $\begin{array}{c}\text { 1,2,3-Trichloro- } \\
\text { propane } \\
(1,2,3-\mathrm{TCP}) \\
(\mu \mathrm{g} / \mathrm{L}) \\
(77443)\end{array}$ & $\begin{array}{c}\text { 1,2-Dibromo- } \\
\text { 3-chloropropane } \\
\text { (DBCP) } \\
(\mu \mathrm{g} / \mathrm{L}) \\
(82625)\end{array}$ & $\begin{array}{c}\text { 1,2-Dibro- } \\
\text { moethane } \\
\text { (EDB) } \\
(\mu \mathrm{g} / \mathrm{L}) \\
(77651)\end{array}$ & $\begin{array}{l}\text { Methyl tert- } \\
\text { butyl ether } \\
\text { (MTBE) } \\
(\mu \mathrm{g} / \mathrm{L}) \\
(78032)\end{array}$ & $\begin{array}{c}\text { tert-butyl alcohol } \\
\text { (TBA; 2-propanol) } \\
(\mu \mathrm{g} / \mathrm{L}) \\
(77035)\end{array}$ & $\begin{array}{c}\text { Chloroform } \\
\text { (trichloro- } \\
\text { methane) } \\
(\mu \mathrm{g} / \mathrm{L}) \\
(32106)\end{array}$ & $\begin{array}{l}\text { Bromodi- } \\
\text { chloro- } \\
\text { methane } \\
(\mu \mathrm{g} / \mathrm{L}) \\
(32101)\end{array}$ & $\begin{array}{c}\text { Butane } \\
(\mu g / L) \\
(81563)\end{array}$ & $\begin{array}{c}\text { Benzene } \\
(\mu \mathrm{g} / \mathrm{L}) \\
(34030)\end{array}$ & $\begin{array}{c}n \text {-Pentane } \\
(\mu \mathrm{g} / \mathrm{L}) \\
(\mathbf{8 1 6 0 4 )}\end{array}$ \\
\hline Benchmark type & MCL-US & HBSL & MCL-US & MCL-US & MCL-CA & NL-CA & MCL-US & MCL-US & na & MCL-CA & na \\
\hline $\begin{array}{l}\text { Benchmark level } \\
(\mu \mathrm{g} / \mathrm{L})\end{array}$ & 5 & 30 & 0.2 & 0.05 & 13 & 12 & 180 & ${ }^{1} 80$ & na & 1 & na \\
\hline$[\mathrm{LRL}]^{2}(\mu \mathrm{g} / \mathrm{L})$ & {$[0.004]$} & {$[0.006]$} & {$[0.02]$} & {$[0.004]$} & {$[0.012]$} & {$[0.24]$} & {$[0.03]$} & {$[0.034]$} & {$[0.038]$} & {$[0.026]$} & {$[0.022]$} \\
\hline
\end{tabular}

Madera/Chowchilla-Kings Shallow Aquifer study unit (77 wells sampled)—Continued

Madera/Chowchilla study area (28 wells sampled)—Continued

Number of wells

with detections

Detection frequency 14

(percent)

Total detections

(number)

Kings study area (49 wells sampled)

\begin{tabular}{|c|c|c|c|c|c|c|c|c|c|c|c|}
\hline S3-MACK-K02 & - & - & - & - & - & - & - & - & - & - & - \\
\hline S3-MACK-K03 & 0.259 & 0.032 & - & - & - & - & - & - & - & - & - \\
\hline S3-MACK-K04 & - & - & - & - & - & - & - & - & - & - & - \\
\hline S3-MACK-K06 & 0.017 & - & - & - & - & - & - & - & - & - & - \\
\hline S3-MACK-K08 & 0.060 & - & - & - & 0.169 & - & - & - & - & - & - \\
\hline S3-MACK-K09 & - & - & - & - & - & - & - & - & - & - $^{(2)}$ & - \\
\hline S3-MACK-K13 & - & - & - & - & - & - & - & - & - & - & - \\
\hline S3-MACK-K14 & - & - & - & - & 0.025 & - & - & - & - & - & - \\
\hline S3-MACK-K15 & - & - & - & - & 0.011 & - & - & - & - & - & - \\
\hline S3-MACK-K16 & 0.103 & 0.084 & - & 0.010 & - & - & - $^{(2)}$ & - & - & - & - \\
\hline
\end{tabular}


Table 5. Volatile organic compounds (VOCs) detected in groundwater samples collected for the Madera/Chowchilla-Kings Shallow Aquifer study unit, Groundwater Ambient Monitoring and Assessment (GAMA) Priority Basin Project, California, August 2013 to April 2014.-Continued

[The five-digit USGS parameter code below the constituent name is used to uniquely identify a specific constituent or property. Samples from all 77 grid wells were analyzed, but only samples with detections are listed unless otherwise noted. Detected constituents are grouped by primary use or source and listed in order of decreasing detection frequency in the 77 grid wells. All constituents are listed in table $3 A$. GAMA well identification numbers: S3-MACK-K, Madera/Chowchilla-Kings Shallow Aquifer Kings study-area grid well; S3-MACK-M, Madera/Chowchilla-Kings Shallow Aquifer Madera/ Chowchilla study-area grid well. Benchmark types: Maximum contaminant level benchmarks are listed as MCL-US when the MCL-US and MCL-CA are identical, and as MCL-CA when the MCL-CA is lower than the MCL-US or no MCL-US exists. HBSL, USGS health-based screening level; MCL-CA; State of California maximum contaminant level; MCL-HI, State of Hawaii maximum contaminant level; MCL-US, U.S. Environmental Protection Agency (EPA) maximum contaminant level; NL-CA, State of California notification level. Benchmark type, benchmark level, and LRL as of April 30, 2014. Abbreviations: LRL, laboratory reporting level; na, not available; SRL, study reporting level; USGS, U.S. Geological Survey; $\mu \mathrm{g} / \mathrm{L}$, micrograms per liter; *, concentration greater than the benchmark level; -, not detected]

\begin{tabular}{|c|c|c|c|c|c|c|c|c|c|c|c|}
\hline \multirow{2}{*}{$\begin{array}{c}\begin{array}{c}\text { Primary use or } \\
\text { source }\end{array} \\
\begin{array}{c}\text { GAMA well } \\
\text { identification } \\
\text { number }\end{array}\end{array}$} & \multicolumn{4}{|c|}{ Fumigants } & \multicolumn{2}{|c|}{ Gasoline oxygenate } & \multicolumn{2}{|c|}{ Trihalomethanes } & \multicolumn{3}{|c|}{ Gasoline hydrocarbons } \\
\hline & $\begin{array}{c}\text { 1,2-Dichloro- } \\
\text { propane } \\
\text { (1,2-DCP) } \\
(\mu \mathrm{g} / \mathrm{L}) \\
(34541)\end{array}$ & $\begin{array}{c}\text { 1,2,3-Trichloro- } \\
\text { propane } \\
(1,2,3-T C P) \\
(\mu \mathrm{g} / \mathrm{L}) \\
(77443)\end{array}$ & $\begin{array}{c}\text { 1,2-Dibromo- } \\
\text { 3-chloropropane } \\
\text { (DBCP) } \\
(\mu \mathrm{g} / \mathrm{L}) \\
(82625)\end{array}$ & $\begin{array}{c}\text { 1,2-Dibro- } \\
\text { moethane } \\
(\mathrm{EDB}) \\
(\mu \mathrm{g} / \mathrm{L}) \\
(77651)\end{array}$ & $\begin{array}{l}\text { Methyl tert- } \\
\text { butyl ether } \\
\text { (MTBE) } \\
(\mu \mathrm{g} / \mathrm{L}) \\
(78032)\end{array}$ & $\begin{array}{c}\text { tert-butyl alcohol } \\
\text { (TBA; 2-propanol) } \\
(\mu \mathrm{g} / \mathrm{L}) \\
(77035)\end{array}$ & $\begin{array}{c}\text { Chloroform } \\
\text { (trichloro- } \\
\text { methane) } \\
(\mu \mathrm{g} / \mathrm{L}) \\
(32106)\end{array}$ & $\begin{array}{c}\text { Bromodi- } \\
\text { chloro- } \\
\text { methane } \\
(\mu \mathrm{g} / \mathrm{L}) \\
(32101)\end{array}$ & $\begin{array}{c}\text { Butane } \\
\text { ( } \mu \mathrm{g} / \mathrm{L}) \\
(81563)\end{array}$ & $\begin{array}{c}\text { Benzene } \\
(\mu \mathrm{g} / \mathrm{L}) \\
(34030)\end{array}$ & $\begin{array}{c}n \text {-Pentane } \\
(\mu \mathrm{g} / \mathrm{L}) \\
(81604)\end{array}$ \\
\hline Benchmark type & MCL-US & HBSL & MCL-US & MCL-US & MCL-CA & NL-CA & MCL-US & MCL-US & na & MCL-CA & na \\
\hline $\begin{array}{l}\text { Benchmark level } \\
(\mu \mathrm{g} / \mathrm{L})\end{array}$ & 5 & 30 & 0.2 & 0.05 & 13 & 12 & 180 & 180 & na & 1 & na \\
\hline$[\mathrm{LRL}]^{2}(\mu \mathrm{g} / \mathrm{L})$ & {$[0.004]$} & {$[0.006]$} & {$[0.02]$} & {$[0.004]$} & {$[0.012]$} & {$[0.24]$} & {$[0.03]$} & {$[0.034]$} & {$[0.038]$} & {$[0.026]$} & {$[0.022]$} \\
\hline
\end{tabular}

Madera/Chowchilla-Kings Shallow Aquifer study unit (77 wells sampled)—Continued

Kings study area (49 wells sampled) - Continued

\begin{tabular}{|c|c|c|c|c|c|c|c|c|c|c|c|}
\hline S3-MACK-K19 & 0.116 & 0.070 & - & - & - & - & 0.13 & - & - & - & - \\
\hline S3-MACK-K20 & 0.004 & 0.469 & - & - & - & - & - & - & - & - & - \\
\hline S3-MACK-K21 & 0.081 & - & - & - & - & - & - & - & - & - & - \\
\hline S3-MACK-K24 & 0.034 & 0.018 & - & $* 0.053$ & - & - & - & - & - & - & - \\
\hline S3-MACK-K25 & 0.030 & 0.032 & 0.12 & - & - & - & - & - & - & - & - \\
\hline S3-MACK-K28 & - & - & - & - & 0.157 & - & - & - & - & - & - \\
\hline S3-MACK-K31 & - & - & - & - & - & - & - & - & 0.041 & 0.032 & 0.017 \\
\hline S3-MACK-K34 & - & - & - & - & 0.132 & - & 0.92 & 0.152 & - & - & - \\
\hline S3-MACK-K35 & - & - & - & - & 0.013 & - & 0.08 & - & $-^{(2)}$ & - & - \\
\hline S3-MACK-K36 & 0.006 & - & - & - & - & - & - & - & - & - & - \\
\hline S3-MACK-K37 & - & - & - & - & - & - & - & - & 0.020 & - & - \\
\hline S3-MACK-K38 & - & 0.185 & 0.01 & - & - & - & - & - & - & - & - \\
\hline S3-MACK-K39 & 0.006 & 0.071 & 0.17 & - & - & - & - & - & - & - & - \\
\hline S3-MACK-K40 & 0.002 & 0.243 & - & - & - & - & - & - & - & - & - \\
\hline S3-MACK-K41 & - & - & 0.15 & - & - & - & - & - & - & - & - \\
\hline
\end{tabular}


Table 5. Volatile organic compounds (VOCs) detected in groundwater samples collected for the Madera/Chowchilla-Kings Shallow Aquifer study unit, Groundwater Ambient Monitoring and Assessment (GAMA) Priority Basin Project, California, August 2013 to April 2014._Continued

[The five-digit USGS parameter code below the constituent name is used to uniquely identify a specific constituent or property. Samples from all 77 grid wells were analyzed, but only samples with detections are listed unless otherwise noted. Detected constituents are grouped by primary use or source and listed in order of decreasing detection frequency in the 77 grid wells. All constituents are listed in table 3A. GAMA well identification numbers: S3-MACK-K, Madera/Chowchilla-Kings Shallow Aquifer Kings study-area grid well; S3-MACK-M, Madera/Chowchilla-Kings Shallow Aquifer Madera/ Chowchilla study-area grid well. Benchmark types: Maximum contaminant level benchmarks are listed as MCL-US when the MCL-US and MCL-CA are identical, and as MCL-CA when the MCL-CA is lower than the MCL-US or no MCL-US exists. HBSL, USGS health-based screening level; MCL-CA; State of California maximum contaminant level; MCL-HI, State of Hawaii maximum contaminant level; MCL-US, U.S. Environmental Protection Agency (EPA) maximum contaminant level; NL-CA, State of California notification level. Benchmark type, benchmark level, and LRL as of April 30, 2014. Abbreviations: LRL, laboratory reporting level; na, not available; SRL, study reporting level; USGS, U.S. Geological Survey; $\mu \mathrm{g} / \mathrm{L}$, micrograms per liter; *, concentration greater than the benchmark level;

- not detected]

\begin{tabular}{|c|c|c|c|c|c|c|c|c|c|c|c|}
\hline \multirow{2}{*}{$\begin{array}{c}\text { Primary use or } \\
\text { source }\end{array}$} & \multicolumn{4}{|c|}{ Fumigants } & \multicolumn{2}{|c|}{ Gasoline oxygenate } & \multicolumn{2}{|c|}{ Trihalomethanes } & \multicolumn{3}{|c|}{ Gasoline hydrocarbons } \\
\hline & $\begin{array}{c}\text { 1,2-Dichloro- } \\
\text { propane } \\
(1,2-\mathrm{DCP}) \\
(\mu \mathrm{g} / \mathrm{L}) \\
(34541)\end{array}$ & $\begin{array}{c}\text { 1,2,3-Trichloro- } \\
\text { propane } \\
(1,2,3-T C P) \\
(\mu \mathrm{g} / \mathrm{L}) \\
(77443)\end{array}$ & $\begin{array}{c}\text { 1,2-Dibromo- } \\
\text { 3-chloropropane } \\
\text { (DBCP) } \\
(\mu \mathrm{g} / \mathrm{L}) \\
(82625)\end{array}$ & $\begin{array}{c}\text { 1,2-Dibro- } \\
\text { moethane } \\
\text { (EDB) } \\
(\mu \mathrm{g} / \mathrm{L}) \\
(77651)\end{array}$ & $\begin{array}{c}\text { Methyl tert- } \\
\text { butyl ether } \\
\text { (MTBE) } \\
(\mu \mathrm{g} / \mathrm{L}) \\
(78032)\end{array}$ & $\begin{array}{c}\text { tert-butyl alcohol } \\
\text { (TBA; 2-propanol) } \\
(\mu \mathrm{g} / \mathrm{L}) \\
(77035)\end{array}$ & 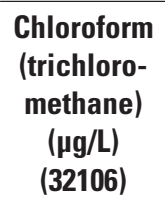 & $\begin{array}{l}\text { Bromodi- } \\
\text { chloro- } \\
\text { methane } \\
(\mu \mathrm{g} / \mathrm{L}) \\
(32101)\end{array}$ & $\begin{array}{c}\text { Butane } \\
\text { ( } \mu \mathrm{gg} / \mathrm{L}) \\
(\mathbf{8 1 5 6 3 )}\end{array}$ & $\begin{array}{c}\text { Benzene } \\
(\mu \mathrm{g} / \mathrm{L}) \\
(\mathbf{3 4 0 3 0})\end{array}$ & $\begin{array}{c}n \text {-Pentane } \\
(\mu \mathrm{g} / \mathrm{L}) \\
(\mathbf{8 1 6 0 4 )}\end{array}$ \\
\hline Benchmark type & MCL-US & HBSL & MCL-US & MCL-US & MCL-CA & NL-CA & MCL-US & MCL-US & na & MCL-CA & na \\
\hline $\begin{array}{l}\text { Benchmark level } \\
\text { ( } \mu \mathrm{g} / \mathrm{L})\end{array}$ & 5 & 30 & 0.2 & 0.05 & 13 & 12 & ${ }^{1} 80$ & ${ }^{1} 80$ & na & 1 & na \\
\hline$[\mathrm{LRL}]^{2}(\mu \mathrm{g} / \mathrm{L})$ & {$[0.004]$} & {$[0.006]$} & {$[0.02]$} & {$[0.004]$} & {$[0.012]$} & {$[0.24]$} & {$[0.03]$} & {$[0.034]$} & {$[0.038]$} & {$[0.026]$} & {$[0.022]$} \\
\hline \multicolumn{12}{|c|}{ Madera/Chowchilla-Kings Shallow Aquifer study unit (77 wells sampled)_Continued } \\
\hline \multicolumn{12}{|c|}{ Kings study area (49 wells sampled)—Continued } \\
\hline S3-MACK-K42 & - & - & - & - & 0.051 & - & -(2) & - & - & - & - \\
\hline S3-MACK-K43 & - & - & - & - & 0.016 & - & - & - & - & - & - \\
\hline S3-MACK-K44 & 0.043 & 1.16 & - & - & - & - & 0.08 & - & - $^{(2)}$ & - & - \\
\hline S3-MACK-K45 & - & - & - & - & - & - & 2.62 & 0.041 & - & - & - \\
\hline S3-MACK-K48 & - & - & - & - & 0.012 & - & - & - & - & - & - \\
\hline S3-MACK-K49 & - & - & - & - & - & - & 0.10 & - & 0.024 & - & - \\
\hline $\begin{array}{l}\text { Number of wells } \\
\text { with detections }\end{array}$ & 13 & 10 & 4 & 2 & 9 & 0 & 6 & 2 & 3 & 1 & 1 \\
\hline $\begin{array}{l}\text { Detection frequency } \\
\text { (percent) }\end{array}$ & 27 & 20 & 8 & 4 & 18 & 0 & 12 & 4 & 6 & 2 & 2 \\
\hline $\begin{array}{l}\text { Total detections } \\
\text { (number) }\end{array}$ & - & - & - & - & - & - & - & - & - & - & - \\
\hline
\end{tabular}


Table 5. Volatile organic compounds (VOCs) detected in groundwater samples collected for the Madera/Chowchilla-Kings Shallow Aquifer study unit, Groundwater Ambient

Monitoring and Assessment (GAMA) Priority Basin Project, California, August 2013 to April 2014.-Continued

[The five-digit USGS parameter code below the constituent name is used to uniquely identify a specific constituent or property. Samples from all 77 grid wells were analyzed, but only samples with detections are listed unless otherwise noted. Detected constituents are grouped by primary use or source and listed in order of decreasing detection frequency in the 77 grid wells. All constituents are listed in table $3 A$. GAMA well identification numbers: S3-MACK-K, Madera/Chowchilla-Kings Shallow Aquifer Kings study-area grid well; S3-MACK-M, Madera/Chowchilla-Kings Shallow Aquifer Madera/ Chowchilla study-area grid well. Benchmark types: Maximum contaminant level benchmarks are listed as MCL-US when the MCL-US and MCL-CA are identical, and as MCL-CA when the MCL-CA is lower than the MCL-US or no MCL-US exists. HBSL, USGS health-based screening level; MCL-CA; State of California maximum contaminant level; MCL-HI, State of Hawaii maximum contaminant level; MCL-US, U.S. Environmental Protection Agency (EPA) maximum contaminant level; NL-CA, State of California notification level. Benchmark type, benchmark level, and LRL as of April 30, 2014. Abbreviations: LRL, laboratory reporting level; na, not available; SRL, study reporting level; USGS, U.S. Geological Survey; $\mu \mathrm{g} / \mathrm{L}$, micrograms per liter; *, concentration greater than the benchmark level; -, not detected]

\begin{tabular}{|c|c|c|c|c|c|c|c|c|}
\hline \multirow{2}{*}{$\begin{array}{l}\text { Primary use or source } \\
\text { GAMA well } \\
\text { identification number }\end{array}$} & \multirow{2}{*}{$\begin{array}{c}\text { Natural } \\
\text { Carbon } \\
\text { disulfide } \\
(\mu \mathrm{g} / \mathrm{L}) \\
(77041) \\
\end{array}$} & \multicolumn{4}{|c|}{ Solvents } & \multirow{2}{*}{$\begin{array}{c}\text { Refrigerant } \\
\text { Chlorodifluoromethane } \\
\text { (HCFC-22) } \\
(\mu \mathrm{g} / \mathrm{L}) \\
(45028)\end{array}$} & \multirow[b]{2}{*}{$\begin{array}{c}\text { Detections } \\
\text { per } \\
\text { well }\end{array}$} & \multirow[b]{2}{*}{$\begin{array}{l}\text { Detection } \\
\text { summary }\end{array}$} \\
\hline & & $\begin{array}{c}\text { Tetrachloroethene } \\
\text { (PCE, PERC) } \\
(\mu \mathrm{g} / \mathrm{L}) \\
(34475)\end{array}$ & $\begin{array}{c}\text { Trichloroethene } \\
(\mathrm{TCE}) \\
(\mu \mathrm{g} / \mathrm{L}) \\
(39180)\end{array}$ & $\begin{array}{c}\text { 1,1-Dichloroethane } \\
(\mu \mathrm{g} / \mathrm{L}) \\
(34496)\end{array}$ & $\begin{array}{c}\text { 1,4-Dioxane } \\
\text { ( } \mu \mathrm{g} / \mathrm{L}) \\
(81582)\end{array}$ & & & \\
\hline Benchmark type & HBSL & MCL-US & MCL-US & MCL-CA & NL-CA & na & - & - \\
\hline Benchmark level & 700 & 5 & 5 & 5 & 3 & na & - & - \\
\hline$[\mathrm{LRL}]^{2}(\mu \mathrm{g} / \mathrm{L})$ & {$[0.1]$} & {$[0.026]$} & {$[0.022]$} & {$[0.044]$} & {$[0.7]$} & {$[0.25]$} & - & - \\
\hline \multicolumn{9}{|c|}{ Madera/Chowchilla-Kings Shallow Aquifer study unit (77 wells sampled)_Continued } \\
\hline $\begin{array}{l}\text { Number of wells with } \\
\text { detections }\end{array}$ & 5 & 5 & 2 & 1 & 1 & 2 & - & 41 \\
\hline $\begin{array}{l}\text { Detection frequency } \\
\text { (percent) }\end{array}$ & 6 & 6 & 3 & 1 & 1 & 3 & - & 53 \\
\hline $\begin{array}{l}\text { Total detections } \\
\text { (number) }\end{array}$ & - & - & - & - & - & - & - & 87 \\
\hline \multicolumn{9}{|c|}{ Madera/Chowchilla study area (28 wells sampled)—Continued } \\
\hline S3-MACK-M08 & - & - & - & - & - & - & 3 & - \\
\hline S3-MACK-M09 & - & - & - & - & - & - & 3 & - \\
\hline S3-MACK-M10 & - & 0.019 & - & - & - & - & 2 & - \\
\hline S3-MACK-M11 & - & - & - & - & - & - & 2 & - \\
\hline S3-MACK-M12 & - & - & - & - & - & - & 1 & - \\
\hline S3-MACK-M14 & - & - & - & - & - & - & 3 & - \\
\hline S3-MACK-M15 & - & - & - & - & - & - & 3 & - \\
\hline S3-MACK-M18 & - & - & - & - & - & - & 1 & - \\
\hline S3-MACK-M19 & - & - & - & - & - & - & 1 & - \\
\hline S3-MACK-M22 & - & - & - & - & - & - & 2 & - \\
\hline
\end{tabular}


Table 5. Volatile organic compounds (VOCs) detected in groundwater samples collected for the Madera/Chowchilla-Kings Shallow Aquifer study unit, Groundwater Ambient Monitoring and Assessment (GAMA) Priority Basin Project, California, August 2013 to April 2014._Continued

[The five-digit USGS parameter code below the constituent name is used to uniquely identify a specific constituent or property. Samples from all 77 grid wells were analyzed, but only samples with detections are listed unless otherwise noted. Detected constituents are grouped by primary use or source and listed in order of decreasing detection frequency in the 77 grid wells. All constituents are listed in table 3A. GAMA well identification numbers: S3-MACK-K, Madera/Chowchilla-Kings Shallow Aquifer Kings study-area grid well; S3-MACK-M, Madera/Chowchilla-Kings Shallow Aquifer Madera/ Chowchilla study-area grid well. Benchmark types: Maximum contaminant level benchmarks are listed as MCL-US when the MCL-US and MCL-CA are identical, and as MCL-CA when the MCL-CA is lower than the MCL-US or no MCL-US exists. HBSL, USGS health-based screening level; MCL-CA; State of California maximum contaminant level; MCL-HI, State of Hawaii maximum contaminant level; MCL-US, U.S. Environmental Protection Agency (EPA) maximum contaminant level; NL-CA, State of California notification level. Benchmark type, benchmark level, and LRL as of April 30, 2014. Abbreviations: LRL, laboratory reporting level; na, not available; SRL, study reporting level; USGS, U.S. Geological Survey; $\mu \mathrm{g} / \mathrm{L}$, micrograms per liter; *, concentration greater than the benchmark level; -, not detected]

\begin{tabular}{|c|c|c|c|c|c|c|c|c|}
\hline \multirow{2}{*}{$\begin{array}{l}\text { Primary use or source } \\
\text { GAMA well } \\
\text { identification number }\end{array}$} & \multirow{2}{*}{$\begin{array}{c}\text { Natural } \\
\text { Carbon } \\
\text { disulfide } \\
(\mu \mathrm{g} / \mathrm{L}) \\
(77041)\end{array}$} & \multicolumn{4}{|c|}{ Solvents } & \multirow{2}{*}{$\begin{array}{c}\text { Refrigerant } \\
\text { Chlorodifluoromethane } \\
\text { (HCFC-22) } \\
(\mu \mathrm{g} / \mathrm{L}) \\
(45028)\end{array}$} & \multirow[b]{2}{*}{$\begin{array}{c}\text { Detections } \\
\text { per } \\
\text { well }\end{array}$} & \multirow[b]{2}{*}{$\begin{array}{l}\text { Detection } \\
\text { summary }\end{array}$} \\
\hline & & $\begin{array}{c}\text { Tetrachloroethene } \\
\text { (PCE, PERC) } \\
(\mu \mathrm{g} / \mathrm{L}) \\
(34475)\end{array}$ & $\begin{array}{c}\text { Trichloroethene } \\
\text { (TCE) } \\
(\mu \mathrm{g} / \mathrm{L}) \\
(39180)\end{array}$ & $\begin{array}{c}\text { 1,1-Dichloroethane } \\
(\mu \mathrm{g} / \mathrm{L}) \\
(34496)\end{array}$ & $\begin{array}{c}\text { 1,4-Dioxane } \\
\text { ( } \mu \mathrm{g} / \mathrm{L}) \\
(81582)\end{array}$ & & & \\
\hline Benchmark type & HBSL & MCL-US & MCL-US & MCL-CA & NL-CA & na & - & - \\
\hline Benchmark level & 700 & 5 & 5 & 5 & 3 & na & - & - \\
\hline$[\mathrm{LRL}]^{2}(\mu \mathrm{g} / \mathrm{L})$ & {$[0.1]$} & {$[0.026]$} & {$[0.022]$} & {$[0.044]$} & {$[0.7]$} & {$[0.25]$} & - & - \\
\hline
\end{tabular}

Madera/Chowchilla-Kings Shallow Aquifer study unit (77 wells sampled)—Continued

Madera/Chowchilla study area (28 wells sampled)—Continued

detections

Detection frequency

0

0

0

(percent)

Total detections

0

36

(number)

Kings study area (49 wells sampled) - Continued

S3-MACK-K03

S3-MACK-K04

S3-MACK-K06

S3-MACK-K08

S3-MACK-K09

S3-MACK-K13

S3-MACK-K14

S3-MACK-K15

S3-MACK-K16

$\begin{array}{cc}0.21 & - \\ -^{(2)} & - \\ 0.43 & - \\ - & - \\ 3.9 & - \\ 0.44 & - \\ 0.24 & - \\ - & -\end{array}$

-

-

$-$

21

$\begin{array}{cllllll}- & - & - & - & - & 1 & - \\ - & - & - & - & - & 2 & - \\ - & - & - & - & - & 1 & - \\ - & - & - & - & - & 1 & - \\ - & - & - & - & - & 2 & - \\ - & - & - & - & - & 1 & - \\ - & - & - & - & - & - & 1 \\ - & - & - & - & - & 3 & -\end{array}$


Table 5. Volatile organic compounds (VOCs) detected in groundwater samples collected for the Madera/Chowchilla-Kings Shallow Aquifer study unit, Groundwater Ambient

Monitoring and Assessment (GAMA) Priority Basin Project, California, August 2013 to April 2014.-Continued

[The five-digit USGS parameter code below the constituent name is used to uniquely identify a specific constituent or property. Samples from all 77 grid wells were analyzed, but only samples with detections are listed unless otherwise noted. Detected constituents are grouped by primary use or source and listed in order of decreasing detection frequency in the 77 grid wells. All constituents are listed in table $3 A$. GAMA well identification numbers: S3-MACK-K, Madera/Chowchilla-Kings Shallow Aquifer Kings study-area grid well; S3-MACK-M, Madera/Chowchilla-Kings Shallow Aquifer Madera/ Chowchilla study-area grid well. Benchmark types: Maximum contaminant level benchmarks are listed as MCL-US when the MCL-US and MCL-CA are identical, and as MCL-CA when the MCL-CA is lower than the MCL-US or no MCL-US exists. HBSL, USGS health-based screening level; MCL-CA; State of California maximum contaminant level; MCL-HI, State of Hawaii maximum contaminant level; MCL-US, U.S. Environmental Protection Agency (EPA) maximum contaminant level; NL-CA, State of California notification level. Benchmark type, benchmark level, and LRL as of April 30, 2014. Abbreviations: LRL, laboratory reporting level; na, not available; SRL, study reporting level; USGS, U.S. Geological Survey; $\mu \mathrm{g} / \mathrm{L}$, micrograms per liter; *, concentration greater than the benchmark level; -, not detected]

\begin{tabular}{|c|c|c|c|c|c|c|c|c|}
\hline \multirow{2}{*}{$\begin{array}{l}\text { Primary use or source } \\
\text { GAMA well } \\
\text { identification number }\end{array}$} & \multirow{2}{*}{$\begin{array}{c}\text { Natural } \\
\text { Carbon } \\
\text { disulfide } \\
(\mu \mathrm{g} / \mathrm{L}) \\
(77041) \\
\end{array}$} & \multicolumn{4}{|c|}{ Solvents } & \multirow{2}{*}{$\begin{array}{c}\text { Refrigerant } \\
\text { Chlorodifluoromethane } \\
\text { (HCFC-22) } \\
(\mu \mathrm{g} / \mathrm{L}) \\
(45028) \\
\end{array}$} & \multirow[b]{2}{*}{$\begin{array}{c}\text { Detections } \\
\text { per } \\
\text { well }\end{array}$} & \multirow[b]{2}{*}{$\begin{array}{l}\text { Detection } \\
\text { summary }\end{array}$} \\
\hline & & $\begin{array}{c}\text { Tetrachloroethene } \\
\text { (PCE, PERC) } \\
(\mu \mathrm{g} / \mathrm{L}) \\
(34475)\end{array}$ & $\begin{array}{c}\text { Trichloroethene } \\
\text { (TCE) } \\
(\mu \mathrm{g} / \mathrm{L}) \\
(39180)\end{array}$ & $\begin{array}{c}\text { 1,1-Dichloroethane } \\
(\mu \mathrm{g} / \mathrm{L}) \\
(34496)\end{array}$ & $\begin{array}{c}\text { 1,4-Dioxane } \\
\text { ( } \mu \mathrm{g} / \mathrm{L}) \\
(81582)\end{array}$ & & & \\
\hline Benchmark type & HBSL & MCL-US & MCL-US & MCL-CA & NL-CA & na & - & - \\
\hline Benchmark level & 700 & 5 & 5 & 5 & 3 & na & - & - \\
\hline$[\mathrm{LRL}]^{2}(\mu \mathrm{g} / \mathrm{L})$ & [0.1] & {$[0.026]$} & [0.022] & [0.044] & [0.7] & [0.25] & - & - \\
\hline \multicolumn{9}{|c|}{ Madera/Chowchilla-Kings Shallow Aquifer study unit (77 wells sampled)_Continued } \\
\hline \multicolumn{9}{|c|}{ Kings study area (49 wells sampled) —Continued } \\
\hline S3-MACK-K19 & - & 0.842 & 0.078 & 0.046 & 1.4 & 1.71 & 8 & - \\
\hline S3-MACK-K20 & - & - & - & - & - & - & 2 & - \\
\hline S3-MACK-K21 & - & - & - & - & - & - & 1 & - \\
\hline S3-MACK-K24 & - & - & - & - & - & - & 3 & - \\
\hline S3-MACK-K25 & - & - & - & - & - & - & 3 & - \\
\hline S3-MACK-K28 & - & - & - & - & - & - & 1 & - \\
\hline S3-MACK-K31 & - & - & - & - & - & - & 3 & - \\
\hline S3-MACK-K34 & - & 0.054 & - & - & - & - $^{(2)}$ & 4 & - \\
\hline S3-MACK-K35 & - & 0.580 & 0.019 & - & - & 0.126 & 5 & - \\
\hline S3-MACK-K36 & - & - & - & - & - & - & 1 & - \\
\hline S3-MACK-K37 & - & - & - & - & - & - & 1 & - \\
\hline S3-MACK-K38 & - & - & - & - & - & - & 2 & - \\
\hline S3-MACK-K39 & - & - & - & - & - & - & 3 & - \\
\hline S3-MACK-K40 & - & - & - & - & - & - & 2 & - \\
\hline S3-MACK-K41 & - & - & - & - & - & - & 1 & - \\
\hline S3-MACK-K42 & - & - & - & - & - & - & 1 & - \\
\hline S3-MACK-K43 & - & - & - & - & - & - & 1 & - \\
\hline
\end{tabular}


Table 5. Volatile organic compounds (VOCs) detected in groundwater samples collected for the Madera/Chowchilla-Kings Shallow Aquifer study unit, Groundwater Ambient Monitoring and Assessment (GAMA) Priority Basin Project, California, August 2013 to April 2014._Continued

[The five-digit USGS parameter code below the constituent name is used to uniquely identify a specific constituent or property. Samples from all 77 grid wells were analyzed, but only samples with detections are listed unless otherwise noted. Detected constituents are grouped by primary use or source and listed in order of decreasing detection frequency in the 77 grid wells. All constituents are listed in table 3A. GAMA well identification numbers: S3-MACK-K, Madera/Chowchilla-Kings Shallow Aquifer Kings study-area grid well; S3-MACK-M, Madera/Chowchilla-Kings Shallow Aquifer Madera/ Chowchilla study-area grid well. Benchmark types: Maximum contaminant level benchmarks are listed as MCL-US when the MCL-US and MCL-CA are identical, and as MCL-CA when the MCL-CA is lower than the MCL-US or no MCL-US exists. HBSL, USGS health-based screening level; MCL-CA; State of California maximum contaminant level; MCL-HI, State of Hawaii maximum contaminant level; MCL-US, U.S. Environmental Protection Agency (EPA) maximum contaminant level; NL-CA, State of California notification level. Benchmark type, benchmark level, and LRL as of April 30, 2014. Abbreviations: LRL, laboratory reporting level; na, not available; SRL, study reporting level; USGS, U.S. Geological Survey; $\mu \mathrm{g} / \mathrm{L}$, micrograms per liter; *, concentration greater than the benchmark level; -, not detected]

\begin{tabular}{|c|c|c|c|c|c|c|c|c|}
\hline \multirow{2}{*}{$\begin{array}{l}\text { Primary use or source } \\
\text { GAMA well } \\
\text { identification number }\end{array}$} & \multirow{2}{*}{$\begin{array}{c}\text { Natural } \\
\text { Carbon } \\
\text { disulfide } \\
(\mu \mathrm{g} / \mathrm{L}) \\
(77041)\end{array}$} & \multicolumn{4}{|c|}{ Solvents } & \multirow{2}{*}{$\begin{array}{c}\text { Refrigerant } \\
\text { Chlorodifluoromethane } \\
\text { (HCFC-22) } \\
(\mu \mathrm{g} / \mathrm{L}) \\
(45028)\end{array}$} & \multirow[b]{2}{*}{$\begin{array}{c}\text { Detections } \\
\text { per } \\
\text { well }\end{array}$} & \multirow[b]{2}{*}{$\begin{array}{l}\text { Detection } \\
\text { summary }\end{array}$} \\
\hline & & $\begin{array}{c}\text { Tetrachloroethene } \\
\text { (PCE, PERC) } \\
(\mu \mathrm{g} / \mathrm{L}) \\
(34475)\end{array}$ & $\begin{array}{c}\text { Trichloroethene } \\
\text { (TCE) } \\
(\mu \mathrm{g} / \mathrm{L}) \\
(39180) \\
\end{array}$ & $\begin{array}{c}\text { 1,1-Dichloroethane } \\
(\mu \mathrm{g} / \mathrm{L}) \\
(34496)\end{array}$ & $\begin{array}{c}\text { 1,4-Dioxane } \\
\text { ( } \mu \mathrm{g} / \mathrm{L}) \\
(81582)\end{array}$ & & & \\
\hline Benchmark type & HBSL & MCL-US & MCL-US & MCL-CA & NL-CA & na & - & - \\
\hline Benchmark level & 700 & 5 & 5 & 5 & 3 & na & - & - \\
\hline$[\mathrm{LRL}]^{2}(\mu \mathrm{g} / \mathrm{L})$ & {$[0.1]$} & {$[0.026]$} & {$[0.022]$} & {$[0.044]$} & {$[0.7]$} & [0.25] & - & - \\
\hline \multicolumn{9}{|c|}{ Madera/Chowchilla-Kings Shallow Aquifer study unit (77 wells sampled)_Continued } \\
\hline \multicolumn{9}{|c|}{ Kings study area (49 wells sampled)_-Continued } \\
\hline S3-MACK-K44 & - & - & - & - & - & - & 3 & - \\
\hline S3-MACK-K45 & - & - & - & - & - & - & 2 & - \\
\hline S3-MACK-K48 & - & 0.014 & - & - & - & - & 2 & - \\
\hline S3-MACK-K49 & - & - & - & - & - & - & 2 & - \\
\hline $\begin{array}{l}\text { Number of wells with } \\
\text { detections }\end{array}$ & 5 & 4 & 2 & 1 & 1 & 2 & - & 31 \\
\hline $\begin{array}{l}\text { Detection frequency } \\
\text { (percent) }\end{array}$ & 10 & 8 & 4 & 2 & 2 & 4 & - & 63 \\
\hline $\begin{array}{l}\text { Total detections } \\
\text { (number) }\end{array}$ & - & - & - & - & - & - & - & 66 \\
\hline
\end{tabular}

${ }^{1}$ The MCL-US threshold for trihalomethanes is for the sum of chloroform, bromoform, bromodichloromethane, and dibromochloromethane.

${ }^{2}$ The LRL is two times the long-term method detection level (LT-MDL). Results at concentrations below the LT-MDL were reported, but were not counted as detections for the purpose of calculating

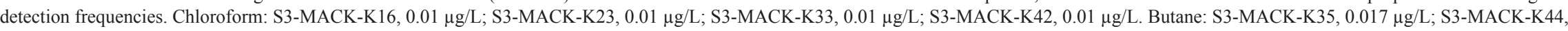
$0.013 \mu \mathrm{g} / \mathrm{L}$. Benzene: S3-MACK-K09, $0.013 \mu \mathrm{g} / \mathrm{L}$. Carbon disulfide: S3-MACK-M01, $0.01 \mu \mathrm{g} / \mathrm{L}$; S3-MACK-K03, $0.01 \mu \mathrm{g} / \mathrm{L} ; \mathrm{S} 3-\mathrm{MACK}-\mathrm{K} 12,0.01 \mu \mathrm{g} / \mathrm{L}$. Cyclohexanone: S3-MACK-M09, 0.46 $\mu \mathrm{g} / \mathrm{L}$. Two additional VOCs were given study reporting levels (SRLs) on the basis of quality-control results; all detections in groundwater samples were at concentrations below the SRLs and, therefore, were not counted

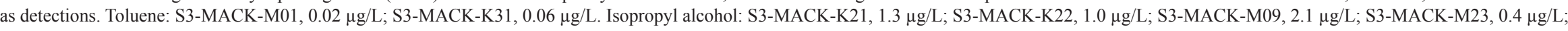
S3-MACK-M26, $0.5 \mu \mathrm{g} / \mathrm{L}$. 
Table 6A. Pesticides detected in groundwater samples collected for the Madera/Chowchilla-Kings Shallow Aquifer study unit, Groundwater Ambient Monitoring and Assessment (GAMA) Priority Basin Project, California, August 2013 to April 2014.

[The five-digit USGS parameter code below the constituent name is used to uniquely identify a specific constituent or property. Samples from 76 grid wells were analyzed, but only samples with detections are listed. All constituents are listed in table 3B. GAMA well identification number: S3-MACK-K, Madera/Chowchilla-Kings Shallow Aquifer Kings study area grid well; S3-MACK-M, Madera/

Chowchilla-Kings Shallow Aquifer Madera/Chowchilla study area grid well. Benchmark type, benchmark level, and LRL as of April 30, 2014. Benchmark levels are reported in the same units as the sample results. Benchmark types: Maximum contaminant level benchmarks are listed as MCL-US when the MCL-US and MCL-CA are identical, and as MCL-CA when the MCL-CA is lower than the MCL-US or no MCL-US exists. HBSL, USGS health-based screening level; HHBP, U.S. Environmental Protection Agency (EPA) Human Health Benchmarks for Pesticides; MCL-CA; State of California maximum contaminant level; MCL-US, EPA maximum contaminant level. Other abbreviations: E, estimated or having a higher degree of uncertainty; LRL, laboratory reporting level; na, not available; ng/L, nanograms per liter; USGS, U.S. Geological Survey; —, not detected]

\begin{tabular}{|c|c|c|c|c|c|c|c|c|c|c|c|c|c|c|}
\hline \multirow{2}{*}{$\begin{array}{l}\text { Primary use or source } \\
\text { GAMA well } \\
\text { identification number }\end{array}$} & \multicolumn{10}{|c|}{ Herbicides } & \multicolumn{2}{|c|}{ Insecticides } & \multirow{5}{*}{$\begin{array}{c}\text { Detections } \\
\text { per } \\
\text { well }\end{array}$} & \multirow{5}{*}{$\begin{array}{l}\text { Detection } \\
\text { summary }\end{array}$} \\
\hline & $\begin{array}{c}\text { Simazine } \\
\text { (ng/L) } \\
(65105)\end{array}$ & $\begin{array}{c}\text { Diuron } \\
\text { (ng/L) } \\
(66598)\end{array}$ & $\begin{array}{c}\text { Norflura- } \\
\text { zon } \\
\text { (ng/L) } \\
(67685)\end{array}$ & $\begin{array}{c}\text { Bromacil } \\
\text { (ng/L) } \\
(68542)\end{array}$ & $\begin{array}{c}\text { Hexazi- } \\
\text { none } \\
\text { (ng/L) } \\
(65085)\end{array}$ & $\begin{array}{c}\text { Atrazine } \\
\text { (ng/L) } \\
(65065)\end{array}$ & $\begin{array}{c}\text { Tebuthi- } \\
\text { uron } \\
\text { (ng/L) } \\
\text { (68695) }\end{array}$ & $\begin{array}{c}\text { Imazethapyr } \\
\text { (ng/L) } \\
(61683)\end{array}$ & $\begin{array}{c}\text { Metola- } \\
\text { chlor } \\
\text { (ng/L) } \\
(65090)\end{array}$ & $\begin{array}{c}\text { Prometon } \\
\text { (ng/L) } \\
(67702)\end{array}$ & $\begin{array}{c}\text { Methoxy- } \\
\text { fenozide } \\
\text { (ng/L) } \\
\text { (68647) }\end{array}$ & $\begin{array}{c}\text { Imidaclo- } \\
\text { prid } \\
\text { (ng/L) } \\
(\mathbf{6 8 4 2 6 )} \\
\end{array}$ & & \\
\hline Benchmark type & MCL-US & HBSL & HHBP & HBSL & HBSL & MCL-CA & HBSL & HHBP & HBSL & HBSL & HHBP & HHBP & & \\
\hline Benchmark level (ng/L) & 4,000 & 20,000 & 105,000 & 700,000 & 400,000 & 1,000 & $1,000,000$ & $17,500,000$ & 700,000 & 400,000 & 714,000 & 399,000 & & \\
\hline [LRL] (ng/L) & [10] & {$[55.6]^{1}$} & {$[5.1]^{1}$} & {$[10]^{1}$} & [3] & {$[15.6]^{2}$} & [3] & {$[25]^{1}$} & [9] & [4] & {$[1.8]^{2}$} & [11] & & \\
\hline \multicolumn{15}{|c|}{ Madera/Chowchilla-Kings Shallow Aquifer study unit (76 wells sampled) ${ }^{3}$} \\
\hline Number of wells with detections & 20 & 7 & 7 & 6 & 5 & 2 & 2 & 1 & 1 & 1 & 2 & 1 & - & 33 \\
\hline Detection frequency (percent) & 26 & 9 & 9 & 8 & 7 & 3 & 3 & 1 & 1 & 1 & 3 & 1 & - & 43 \\
\hline Total detections (number) & - & - & - & - & - & - & - & - & - & - & - & - & - & 55 \\
\hline \multicolumn{15}{|c|}{ Madera/Chowchilla study area (27 wells sampled) ${ }^{3}$} \\
\hline S3-MACK-M01 & 18.2 & - & 10.3 & - & - & - & - & - & - & - & - & - & 2 & - \\
\hline S3-MACK-M07 & - & - & 18.4 & - & - & - & - & - & - & - & - & - & 1 & - \\
\hline S3-MACK-M08 & - & - & - & 25.3 & - & - & - & - & - & - & - & - & 1 & - \\
\hline S3-MACK-M14 & - & - & - & 29.5 & - & 51.3 & - & - & 4.5 & - & - & - & 3 & - \\
\hline S3-MACK-M15 & - & - & - & - & - & 18.7 & - & - & - & - & - & - & 1 & - \\
\hline S3-MACK-M22 & - & - & - & 8.7 & - & - & - & - & - & - & - & - & 1 & - \\
\hline S3-MACK-M23 & 636 & - & - & - & - & - & - & - & - & - & - & - & 1 & - \\
\hline Number of wells with detections & 2 & 0 & 2 & 3 & 0 & 2 & 0 & 0 & 1 & 0 & 0 & 0 & - & 7 \\
\hline Detection frequency (percent) & 7 & 0 & 7 & 11 & 0 & 7 & 0 & 0 & 4 & 0 & 0 & 0 & - & 26 \\
\hline Total detections (number) & - & - & - & - & - & - & - & - & - & - & - & - & - & 10 \\
\hline \multicolumn{15}{|l|}{ Kings study area (49 wells sampled) } \\
\hline S3-MACK-K01 & - & - & - & - & 23.8 & - & - & - & - & - & - & - & 1 & - \\
\hline S3-MACK-K02 & - & 30.4 & - & - & - & - & - & - & - & - & - & - & 1 & - \\
\hline S3-MACK-K11 & - & - & - & - & 1.5 & - & - & - & - & - & - & - & 1 & - \\
\hline
\end{tabular}


Table 6A. Pesticides detected in groundwater samples collected for the Madera/Chowchilla-Kings Shallow Aquifer study unit, Groundwater Ambient Monitoring and Assessment (GAMA) Priority Basin Project, California, August 2013 to April 2014._Continued

[The five-digit USGS parameter code below the constituent name is used to uniquely identify a specific constituent or property. Samples from 76 grid wells were analyzed, but only samples with detections are listed. All constituents are listed in table 3B. GAMA well identification number: S3-MACK-K, Madera/Chowchilla-Kings Shallow Aquifer Kings study area grid well; S3-MACK-M, Madera/ Chowchilla-Kings Shallow Aquifer Madera/Chowchilla study area grid well. Benchmark type, benchmark level, and LRL as of April 30, 2014. Benchmark levels are reported in the same units as the sample results. Benchmark types: Maximum contaminant level benchmarks are listed as MCL-US when the MCL-US and MCL-CA are identical, and as MCL-CA when the MCL-CA is lower than the MCL-US or no MCL-US exists. HBSL, USGS health-based screening level; HHBP, U.S. Environmental Protection Agency (EPA) Human Health Benchmarks for Pesticides; MCL-CA; State of California maximum contaminant level; MCL-US, EPA maximum contaminant level. Other abbreviations: E, estimated or having a higher degree of uncertainty; LRL, laboratory reporting level; na, not available; ng/L, nanograms per liter; USGS, U.S. Geological Survey; - , not detected]

\begin{tabular}{|c|c|c|c|c|c|c|c|c|c|c|c|c|c|c|}
\hline Primary use or source & \multicolumn{10}{|c|}{ Herbicides } & \multicolumn{2}{|c|}{ Insecticides } & \multirow{5}{*}{$\begin{array}{c}\text { Detections } \\
\text { per } \\
\text { well }\end{array}$} & \multirow{5}{*}{$\begin{array}{l}\text { Detection } \\
\text { summary }\end{array}$} \\
\hline $\begin{array}{l}\text { GAMA well } \\
\text { identification number }\end{array}$ & $\begin{array}{c}\text { Simazine } \\
\text { (ng/L) } \\
(65105)\end{array}$ & $\begin{array}{c}\text { Diuron } \\
\text { (ng/L) } \\
(66598)\end{array}$ & $\begin{array}{l}\text { Norflura- } \\
\text { zon } \\
\text { (ng/L) } \\
(67685)\end{array}$ & $\begin{array}{c}\text { Bromacil } \\
\text { (ng/L) } \\
(68542)\end{array}$ & $\begin{array}{c}\text { Hexazi- } \\
\text { none } \\
\text { (ng/L) } \\
(65085)\end{array}$ & $\begin{array}{c}\text { Atrazine } \\
\text { (ng/L) } \\
(65065)\end{array}$ & $\begin{array}{l}\text { Tebuthi- } \\
\text { uron } \\
\text { (ng/L) } \\
(68695)\end{array}$ & $\begin{array}{c}\text { Imazethapyr } \\
\text { (ng/L) } \\
(61683)\end{array}$ & $\begin{array}{c}\text { Metola- } \\
\text { chlor } \\
\text { (ng/L) } \\
(65090)\end{array}$ & $\begin{array}{c}\text { Prometon } \\
\text { (ng/L) } \\
(67702)\end{array}$ & $\begin{array}{c}\text { Methoxy- } \\
\text { fenozide } \\
\text { (ng/L) } \\
\text { (68647) }\end{array}$ & $\begin{array}{c}\text { Imidaclo- } \\
\text { prid } \\
\text { (ng/L) } \\
(68426)\end{array}$ & & \\
\hline Benchmark type & MCL-US & HBSL & HHBP & HBSL & HBSL & MCL-CA & HBSL & HHBP & HBSL & HBSL & HHBP & HHBP & & \\
\hline Benchmark level (ng/L) & 4,000 & 20,000 & 105,000 & 700,000 & 400,000 & 1,000 & $1,000,000$ & $17,500,000$ & 700,000 & 400,000 & 714,000 & 399,000 & & \\
\hline [LRL] (ng/L) & {$[10]$} & {$[55.6]^{1}$} & {$[5.1]^{1}$} & {$[10]^{1}$} & [3] & {$[15.6]^{2}$} & [3] & {$[25]^{1}$} & [9] & [4] & {$[1.8]^{2}$} & [11] & & \\
\hline
\end{tabular}

Madera/Chowchilla-Kings Shallow Aquifer study unit (76 wells sampled) ${ }^{3}$-Continued

Kings study area (49 wells sampled)-Continued

\begin{tabular}{|c|c|c|c|c|c|c|c|c|c|c|c|c|c|c|}
\hline S3-MACK-K13 & - & - & - & - & 43.6 & - & 5.4 & - & - & 9.2 & - & - & 3 & - \\
\hline S3-MACK-K15 & - & E151 & - & - & - & - & - & - & - & - & - & - & 1 & - \\
\hline S3-MACK-K16 & 20.4 & - & - & - & 34.3 & - & - & E40.8 & - & - & - & - & 3 & - \\
\hline S3-MACK-K17 & 34.1 & - & - & - & - & - & - & - & - & - & - & - & 1 & - \\
\hline S3-MACK-K18 & - & - & - & - & 1.8 & - & - & - & - & - & - & - & 1 & - \\
\hline S3-MACK-K20 & 17.2 & - & - & - & - & - & - & - & - & - & - & - & 1 & - \\
\hline S3-MACK-K25 & 51.4 & - & - & - & - & - & - & - & - & - & - & - & 1 & - \\
\hline S3-MACK-K26 & 31.2 & - & - & - & - & - & - & - & - & - & - & - & 1 & - \\
\hline S3-MACK-K27 & 16.5 & - & - & - & - & - & - & - & - & - & - & - & 1 & - \\
\hline S3-MACK-K28 & 9.1 & - & - & - & - & - & - & - & - & - & - & - & 1 & - \\
\hline S3-MACK-K30 & 25.8 & - & - & - & - & - & - & - & - & - & - & - & 1 & - \\
\hline S3-MACK-K32 & 60.7 & - & - & - & - & - & - & - & - & - & - & - & 1 & - \\
\hline S3-MACK-K33 & 39.3 & 79.5 & 4.7 & - & - & - & - & - & - & - & - & 6.2 & 4 & - \\
\hline S3-MACK-K35 & 27.7 & 42.4 & - & 18.4 & - & - & - & - & - & - & - & - & 3 & - \\
\hline S3-MACK-K37 & 94.2 & 131 & 2.9 & - & - & - & 8.6 & - & - & - & 5.8 & - & 5 & - \\
\hline S3-MACK-K38 & 81.1 & 90.3 & 2.6 & - & - & - & - & - & - & - & - & - & 3 & - \\
\hline S3-MACK-K40 & 8.7 & - & - & - & - & - & - & - & - & - & - & - & 1 & - \\
\hline
\end{tabular}


Table 6A. Pesticides detected in groundwater samples collected for the Madera/Chowchilla-Kings Shallow Aquifer study unit, Groundwater Ambient Monitoring and Assessment (GAMA) Priority Basin Project, California, August 2013 to April 2014.-Continued

[The five-digit USGS parameter code below the constituent name is used to uniquely identify a specific constituent or property. Samples from 76 grid wells were analyzed, but only samples with detections are listed. All constituents are listed in table 3B. GAMA well identification number: S3-MACK-K, Madera/Chowchilla-Kings Shallow Aquifer Kings study area grid well; S3-MACK-M, Madera/

Chowchilla-Kings Shallow Aquifer Madera/Chowchilla study area grid well. Benchmark type, benchmark level, and LRL as of April 30, 2014. Benchmark levels are reported in the same units as the sample results. Benchmark types: Maximum contaminant level benchmarks are listed as MCL-US when the MCL-US and MCL-CA are identical, and as MCL-CA when the MCL-CA is lower than the MCL-US or no MCL-US exists. HBSL, USGS health-based screening level; HHBP, U.S. Environmental Protection Agency (EPA) Human Health Benchmarks for Pesticides; MCL-CA; State of California maximum contaminant level; MCL-US, EPA maximum contaminant level. Other abbreviations: E, estimated or having a higher degree of uncertainty; LRL, laboratory reporting level; na, not available; ng/L, nanograms per liter; USGS, U.S. Geological Survey; —, not detected]

\begin{tabular}{|c|c|c|c|c|c|c|c|c|c|c|c|c|c|c|}
\hline Primary use or source & \multicolumn{10}{|c|}{ Herbicides } & \multicolumn{2}{|c|}{ Insecticides } & \multirow{5}{*}{$\begin{array}{c}\text { Detections } \\
\text { per } \\
\text { well }\end{array}$} & \multirow{5}{*}{$\begin{array}{l}\text { Detection } \\
\text { summary }\end{array}$} \\
\hline $\begin{array}{c}\text { GAMA well } \\
\text { identification number }\end{array}$ & $\begin{array}{c}\text { Simazine } \\
\text { (ng/L) } \\
(65105)\end{array}$ & $\begin{array}{c}\text { Diuron } \\
\text { (ng/L) } \\
(66598)\end{array}$ & $\begin{array}{l}\text { Norflura- } \\
\text { zon } \\
\text { (ng/L) } \\
(67685)\end{array}$ & $\begin{array}{c}\text { Bromacil } \\
\text { (ng/L) } \\
(68542)\end{array}$ & $\begin{array}{c}\text { Hexazi- } \\
\text { none } \\
\text { (ng/L) } \\
(65085)\end{array}$ & $\begin{array}{c}\text { Atrazine } \\
\text { (ng/L) } \\
(65065)\end{array}$ & $\begin{array}{l}\text { Tebuthi- } \\
\text { uron } \\
\text { (ng/L) } \\
(\mathbf{6 8 6 9 5 )}\end{array}$ & $\begin{array}{c}\text { Imazethapyr } \\
\text { (ng/L) } \\
(61683)\end{array}$ & $\begin{array}{c}\text { Metola- } \\
\text { chlor } \\
\text { (ng/L) } \\
(65090)\end{array}$ & $\begin{array}{c}\text { Prometon } \\
\text { (ng/L) } \\
(67702)\end{array}$ & $\begin{array}{c}\text { Methoxy- } \\
\text { fenozide } \\
\text { (ng/L) } \\
\text { (68647) }\end{array}$ & $\begin{array}{c}\text { Imidaclo- } \\
\text { prid } \\
\text { (ng/L) } \\
(68426)\end{array}$ & & \\
\hline Benchmark type & MCL-US & HBSL & HHBP & HBSL & HBSL & MCL-CA & HBSL & HHBP & HBSL & HBSL & HHBP & HHBP & & \\
\hline Benchmark level (ng/L) & 4,000 & 20,000 & 105,000 & 700,000 & 400,000 & 1,000 & $1,000,000$ & $17,500,000$ & 700,000 & 400,000 & 714,000 & 399,000 & & \\
\hline [LRL] (ng/L) & [10] & {$[55.6]^{1}$} & {$[5.1]^{1}$} & {$[10]^{1}$} & [3] & {$[15.6]^{2}$} & [3] & {$[25]^{1}$} & [9] & [4] & {$[1.8]^{2}$} & [11] & & \\
\hline
\end{tabular}

Madera/Chowchilla-Kings Shallow Aquifer study unit (76 wells sampled) ${ }^{3}$-Continued

Kings study area (49 wells sampled)-Continued

\begin{tabular}{|c|c|c|c|c|c|c|c|c|c|c|c|c|c|c|}
\hline S3-MACK-K41 & - & - & 3.1 & - & - & - & - & - & - & - & - & - & 1 & - \\
\hline S3-MACK-K43 & 24.1 & - & - & 51.6 & - & - & - & - & - & - & - & - & 2 & - \\
\hline S3-MACK-K44 & 40.8 & - & 6.1 & 283 & - & - & - & - & - & - & - & - & 3 & - \\
\hline S3-MACK-K45 & 40.6 & 51.5 & - & - & - & - & - & - & - & - & - & - & 2 & - \\
\hline S3-MACK-K47 & 52.3 & - & - & - & - & - & - & - & - & - & - & - & 1 & - \\
\hline S3-MACK-K49 & - & - & - & - & - & - & - & - & - & - & 5.5 & - & 1 & - \\
\hline Number of wells with detections & 18 & 7 & 5 & 3 & 5 & 0 & 2 & 1 & 0 & 1 & 2 & 1 & - & 26 \\
\hline Detection frequency (percent) & 37 & 14 & 10 & 6 & 10 & 0 & 4 & 2 & 0 & 2 & 4 & 2 & - & 53 \\
\hline Total detections (number) & - & - & - & - & - & - & - & - & - & - & - & - & - & 45 \\
\hline
\end{tabular}

${ }^{1}$ The LRL for some constituents changed during the study. The highest LRL was used. The LRL is two times the long-term method detection level (LT-MDL), and detections at concentrations below the highest LT-MDL are not reported and are not counted as detections for the purpose of calculating detection frequencies.

${ }^{2}$ Atrazine and methoxyfenozide were assigned interim study reporting levels (iSRLs) based on detections in laboratory prep blanks. Detections in groundwater samples at concentrations less than the iSRLs are not reported and are not counted as detections for the purpose of calculating detection frequencies.

${ }^{3}$ Results are not available for one sample in the Madera/Chowchilla study area: S3-MACK-20 (see appendix). 
Table 6B. Pesticide degradates detected in samples collected for the Madera/Chowchilla-Kings Shallow Aquifer study unit, Groundwater Ambient Monitoring and Assessment (GAMA) Priority Basin Project, California, August 2013 to April 2014.

[The five-digit USGS parameter code below the constituent name is used to uniquely identify a specific constituent or property. Samples from 76 grid wells were analyzed, but only samples with detections are listed. All constituents are listed in table 3B. GAMA well identification number: S3-MACK-K, Madera/Chowchilla-Kings Shallow Aquifer Kings study area grid well; S3-MACK-M, Madera/ChowchillaKings Shallow Aquifer Madera/Chowchilla study area grid well. Benchmark type, benchmark level, and LRL as of April 30, 2014. Benchmark levels are reported in the same units as the sample results. Benchmark type: HHBP, U.S. Environmental Protection Agency (EPA) Human Health Benchmarks for Pesticides. Abbreviations: E, estimated or having a higher degree of uncertainty; LRL, laboratory reporting level; na, not available; ng/L, nanograms per liter; USGS, U.S. Geological Survey; —, not detected]

\begin{tabular}{|c|c|c|c|c|c|c|c|c|c|c|c|c|c|}
\hline \multirow{2}{*}{$\begin{array}{l}\text { Primary use or source } \\
\text { GAMA well } \\
\text { identification number }\end{array}$} & \multicolumn{10}{|c|}{ Herbicide degradates } & \multirow{2}{*}{ 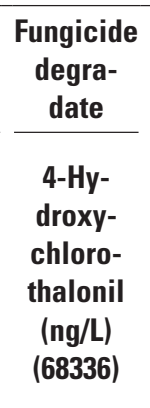 } & \multirow[b]{2}{*}{$\begin{array}{c}\text { Detec- } \\
\text { tions } \\
\text { per } \\
\text { well }\end{array}$} & \multirow[b]{2}{*}{$\begin{array}{l}\text { Detection } \\
\text { summary }\end{array}$} \\
\hline & $\begin{array}{l}\text { Chloro- } \\
\text { diamino- } \\
\text { s-triazine } \\
\text { (CAAT) } \\
\text { (ng/L) } \\
\text { (68547) }\end{array}$ & $\begin{array}{l}\text { 2-Chloro- } \\
\text { 6-ethylamino- } \\
\text { 4-amino-s- } \\
\text { triazine } \\
\text { (CEAT) } \\
\text { (ng/L) } \\
\text { (68550) }\end{array}$ & $\begin{array}{l}{ }^{2} \\
\text { - Demethyl } \\
\text { norflura- } \\
\text { zon } \\
\text { (ng/L) } \\
\text { (68567) }\end{array}$ & $\begin{array}{l}\text { 2-Chloro-4-iso- } \\
\text { propylamino- } \\
\text { 6-amino-s- } \\
\text { triazine } \\
\text { (CIAT) } \\
\text { (ng/L) } \\
\text { (68552) }\end{array}$ & $\begin{array}{l}\text { Demethyl } \\
\text { hexazi- } \\
\text { none B } \\
\text { (ng/L) } \\
\text { (68566) }\end{array}$ & $\begin{array}{l}\text { Metola- } \\
\text { chlor } \\
\text { sulfonic } \\
\text { acid } \\
\text { (ng/L) } \\
\text { (68651) }\end{array}$ & $\begin{array}{l}\text { Metola- } \\
\text { chlor } \\
\text { oxanilic } \\
\text { acid } \\
\text { (ng/L) } \\
\text { (68650) }\end{array}$ & $\begin{array}{l}\text { Dechlo- } \\
\text { rometola- } \\
\text { chlor } \\
\text { (ng/L) } \\
\text { (68562) }\end{array}$ & $\begin{array}{l}\text { 2-Hydroxy-4-iso- } \\
\text { propylamino- } \\
\text { 6-ethylamino-s- } \\
\text { triazine } \\
\text { (OIET) } \\
\text { (ng/L) } \\
\text { (68660) }\end{array}$ & $\begin{array}{c}\text { Hydroxy- } \\
\text { metola- } \\
\text { chlor } \\
\text { (ng/L) } \\
\text { (68622) }\end{array}$ & & & \\
\hline Benchmark type & HHBP & na & na & na & na & na & na & na & HHBP & na & na & - & - \\
\hline Benchmark level (ng/L) & 126,000 & na & na & na & na & na & na & na & 70,000 & na & na & - & - \\
\hline [LRL] (ng/L) & {$[65]^{1}$} & {$[50]^{1}$} & {$[4.1]^{1}$} & [11] & [3] & [68] & [149] & {$[5]^{1}$} & [8] & {$[5]^{1}$} & {$[100]^{1}$} & - & - \\
\hline \multicolumn{14}{|c|}{ Madera/Chowchilla-Kings Shallow Aquifer study unit (76 wells sampled) ${ }^{2}$} \\
\hline Number of wells with detections & 26 & 17 & 14 & 9 & 5 & 3 & 2 & 1 & 1 & 1 & 1 & - & 42 \\
\hline Detection frequency (percent) & 34 & 22 & 18 & 12 & 7 & 4 & 3 & 1 & 1 & 1 & 1 & - & 55 \\
\hline Total detections (number) & - & - & - & - & - & - & - & - & - & - & - & - & 80 \\
\hline \multicolumn{14}{|c|}{ Madera/Chowchilla study area (27 wells sampled) ${ }^{2}$} \\
\hline S3-MACK-M01 & - & - & 2.4 & - & - & - & - & - & - & - & - & 1 & - \\
\hline S3-MACK-M03 & - & - & 61.7 & - & - & - & - & - & - & - & - & 1 & - \\
\hline S3-MACK-M07 & 94.5 & 60.9 & 91.1 & - & - & - & - & - & - & - & - & 3 & - \\
\hline S3-MACK-M08 & 187 & - & - & - & - & - & - & - & - & - & - & 1 & - \\
\hline S3-MACK-M09 & - & - & - & 6.9 & - & - & - & - & - & - & - & 1 & - \\
\hline S3-MACK-M11 & - & 31.8 & - & 21.8 & - & - & - & - & - & - & - & 2 & - \\
\hline S3-MACK-M12 & 43.5 & - & - & - & - & - & - & - & - & - & - & 1 & - \\
\hline S3-MACK-M13 & - & - & - & 8.2 & - & - & - & - & - & - & - & 1 & - \\
\hline S3-MACK-M14 & - & - & - & E106 & - & - & - & - & - & - & - & 1 & - \\
\hline S3-MACK-M15 & - & - & - & E70.7 & - & - & - & - & - & - & - & 1 & - \\
\hline S3-MACK-M17 & - & - & - & - & 1.6 & - & - & - & - & - & - & 1 & - \\
\hline S3-MACK-M22 & - & - & - & 9.5 & - & - & - & - & - & - & - & 1 & - \\
\hline
\end{tabular}


Table 6B. Pesticide degradates detected in samples collected for the Madera/Chowchilla-Kings Shallow Aquifer study unit, Groundwater Ambient Monitoring and Assessment (GAMA) Priority Basin Project, California, August 2013 to April 2014.-Continued

[The five-digit USGS parameter code below the constituent name is used to uniquely identify a specific constituent or property. Samples from 76 grid wells were analyzed, but only samples with detections are listed. All constituents are listed in table 3B. GAMA well identification number: S3-MACK-K, Madera/Chowchilla-Kings Shallow Aquifer Kings study area grid well; S3-MACK-M, Madera/Chowchilla-

Kings Shallow Aquifer Madera/Chowchilla study area grid well. Benchmark type, benchmark level, and LRL as of April 30, 2014. Benchmark levels are reported in the same units as the sample results. Benchmark type: HHBP, U.S. Environmental Protection Agency (EPA) Human Health Benchmarks for Pesticides. Abbreviations: E, estimated or having a higher degree of uncertainty; LRL, laboratory reporting level; na, not available; ng/L, nanograms per liter; USGS, U.S. Geological Survey; —, not detected]

\begin{tabular}{|c|c|c|c|c|c|c|c|c|c|c|c|c|c|}
\hline \multirow{2}{*}{$\begin{array}{c}\text { Primary use or source } \\
\\
\text { GAMA well } \\
\text { identification number }\end{array}$} & \multicolumn{10}{|c|}{ Herbicide degradates } & \multirow{2}{*}{$\begin{array}{c}\begin{array}{c}\text { Fungicide } \\
\text { degra- } \\
\text { date }\end{array} \\
\text { 4-Hy- } \\
\text { droxy- } \\
\text { chloro- } \\
\text { thalonil } \\
\text { (ng/L) } \\
(68336)\end{array}$} & \multirow[b]{2}{*}{$\begin{array}{c}\text { Detec- } \\
\text { tions } \\
\text { per } \\
\text { well }\end{array}$} & \multirow[b]{2}{*}{$\begin{array}{l}\text { Detection } \\
\text { summary }\end{array}$} \\
\hline & $\begin{array}{l}\text { Chloro- } \\
\text { diamino- } \\
\text { s-triazine } \\
\text { (CAAT) } \\
\text { (ng/L) } \\
\text { (68547) }\end{array}$ & $\begin{array}{l}\text { 2-Chloro- } \\
\text { 6-ethylamino- } \\
\text { 4-amino-s- } \\
\text { triazine } \\
\text { (CEAT) } \\
\text { (ng/L) } \\
\text { (68550) }\end{array}$ & $\begin{array}{l}\text { - Demethyl } \\
\text { norflura- } \\
\text { zon } \\
\text { (ng/L) } \\
\text { (68567) }\end{array}$ & $\begin{array}{l}\text { 2-Chloro-4-iso- } \\
\text { propylamino- } \\
\text { 6-amino-s- } \\
\text { triazine } \\
\text { (CIAT) } \\
\text { (ng/L) } \\
(68552)\end{array}$ & $\begin{array}{l}\text { Demethyl } \\
\text { hexazi- } \\
\text { none B } \\
\text { (ng/L) } \\
\text { (68566) }\end{array}$ & $\begin{array}{l}\text { Metola- } \\
\text { chlor } \\
\text { sulfonic } \\
\text { acid } \\
\text { (ng/L) } \\
\text { (68651) }\end{array}$ & $\begin{array}{l}\text { Metola- } \\
\text { chlor } \\
\text { oxanilic } \\
\text { acid } \\
\text { (ng/L) } \\
\text { (68650) }\end{array}$ & $\begin{array}{l}\text { Dechlo- } \\
\text { rometola- } \\
\text { chlor } \\
\text { (ng/L) } \\
\text { (68562) }\end{array}$ & $\begin{array}{c}\text { 2-Hydroxy-4-iso- } \\
\text { propylamino- } \\
\text { - 6-ethylamino-s- } \\
\text { triazine } \\
\text { (OIET) } \\
\text { (ng/L) } \\
(68660)\end{array}$ & $\begin{array}{c}\text { Hydroxy- } \\
\text { metola- } \\
\text { chlor } \\
\text { (ng/L) } \\
\text { (68622) }\end{array}$ & & & \\
\hline Benchmark type & HHBP & na & na & na & na & na & na & na & HHBP & na & na & - & - \\
\hline Benchmark level (ng/L) & 126,000 & na & na & na & na & na & na & na & 70,000 & na & na & - & - \\
\hline$[\mathrm{LRL}](\mathrm{ng} / \mathrm{L})$ & {$[65]^{1}$} & {$[50]^{1}$} & {$[4.1]^{1}$} & [11] & [3] & [68] & [149] & {$[5]^{1}$} & {$[8]$} & {$[5]^{1}$} & {$[100]^{1}$} & - & - \\
\hline \multicolumn{14}{|c|}{ Madera/Chowchilla-Kings Shallow Aquifer study unit (76 wells sampled) ${ }^{2}$-Continued } \\
\hline \multicolumn{14}{|c|}{ Madera/Chowchilla study area (27 wells sampled)²_Continued } \\
\hline Number of wells with detections & 3 & 2 & 3 & 6 & 1 & 0 & 0 & 0 & 0 & 0 & 0 & - & 12 \\
\hline Detection frequency (percent) & 11 & 7 & 11 & 22 & 4 & 0 & 0 & 0 & 0 & 0 & 0 & - & 44 \\
\hline Total detections (number) & - & - & - & - & - & - & - & - & - & - & - & - & 15 \\
\hline \multicolumn{14}{|l|}{ Kings study area (49 wells sampled) } \\
\hline S3-MACK-K01 & - & - & - & - & 10.3 & 1,630 & 238 & 37.9 & - & 5.6 & - & 5 & - \\
\hline S3-MACK-K07 & 101 & - & - & - & - & - & - & - & 5.4 & - & - & 2 & - \\
\hline S3-MACK-K08 & 176 & - & - & - & - & - & - & - & - & - & - & 1 & - \\
\hline S3-MACK-K10 & 835 & - & - & - & - & - & - & - & - & - & - & 1 & - \\
\hline S3-MACK-K13 & - & - & 3.3 & - & 12.7 & 1,110 & 151 & - & - & - & - & 4 & - \\
\hline S3-MACK-K16 & 459 & 85.9 & 2.5 & - & 30.1 & - & - & - & - & - & - & 4 & - \\
\hline S3-MACK-K18 & - & - & - & - & 7.5 & 96.6 & - & - & - & - & - & 2 & - \\
\hline S3-MACK-K19 & 282 & - & - & - & - & - & - & - & - & - & - & 1 & - \\
\hline S3-MACK-K20 & 92.8 & - & - & - & - & - & - & - & - & - & - & 1 & - \\
\hline S3-MACK-K23 & - & 30.1 & - & - & - & - & - & - & - & - & - & 1 & - \\
\hline S3-MACK-K25 & 166 & 126 & 5.4 & - & - & - & - & - & - & - & - & 3 & - \\
\hline S3-MACK-K26 & 61.6 & - & 22 & - & - & - & - & - & - & - & - & 2 & - \\
\hline
\end{tabular}


Table 6B. Pesticide degradates detected in samples collected for the Madera/Chowchilla-Kings Shallow Aquifer study unit, Groundwater Ambient Monitoring and Assessment (GAMA) Priority Basin Project, California, August 2013 to April 2014.-Continued

[The five-digit USGS parameter code below the constituent name is used to uniquely identify a specific constituent or property. Samples from 76 grid wells were analyzed, but only samples with detections are listed. All constituents are listed in table 3B. GAMA well identification number: S3-MACK-K, Madera/Chowchilla-Kings Shallow Aquifer Kings study area grid well; S3-MACK-M, Madera/ChowchillaKings Shallow Aquifer Madera/Chowchilla study area grid well. Benchmark type, benchmark level, and LRL as of April 30, 2014. Benchmark levels are reported in the same units as the sample results.

Benchmark type: HHBP, U.S. Environmental Protection Agency (EPA) Human Health Benchmarks for Pesticides. Abbreviations: E, estimated or having a higher degree of uncertainty; LRL, laboratory reporting level; na, not available; ng/L, nanograms per liter; USGS, U.S. Geological Survey; —, not detected]

\begin{tabular}{|c|c|c|c|c|c|c|c|c|c|c|c|c|c|}
\hline \multirow{2}{*}{$\begin{array}{c}\text { Primary use or source } \\
\text { GAMA well } \\
\text { identification number }\end{array}$} & \multicolumn{10}{|c|}{ Herbicide degradates } & \multirow{2}{*}{$\begin{array}{c}\begin{array}{c}\text { Fungicide } \\
\text { degra- } \\
\text { date }\end{array} \\
\text { 4-Hy- } \\
\text { droxy- } \\
\text { chloro- } \\
\text { thalonil } \\
\text { (ng/L) } \\
\text { (68336) }\end{array}$} & \multirow[b]{2}{*}{$\begin{array}{c}\text { Detec- } \\
\text { tions } \\
\text { per } \\
\text { well }\end{array}$} & \multirow[b]{2}{*}{$\begin{array}{l}\text { Detection } \\
\text { summary }\end{array}$} \\
\hline & $\begin{array}{l}\text { Chloro- } \\
\text { diamino- } \\
\text { s-triazine } \\
\text { (CAAT) } \\
\text { (ng/L) } \\
(68547)\end{array}$ & $\begin{array}{l}\text { 2-Chloro- } \\
\text { 6-ethylamino- } \\
\text { 4-amino-s- } \\
\text { triazine } \\
\text { (CEAT) } \\
\text { (ng/L) } \\
\text { (68550) }\end{array}$ & $\begin{array}{l}\text { Demethyl } \\
\text { norflura- } \\
\text { zon } \\
\text { (ng/L) } \\
\text { (68567) }\end{array}$ & $\begin{array}{l}\text { 2-Chloro-4-iso- } \\
\text { propylamino- } \\
\text { 6-amino-s- } \\
\text { triazine } \\
\text { (CIAT) } \\
\text { (ng/L) } \\
\text { (68552) }\end{array}$ & $\begin{array}{l}\text { Demethyl } \\
\text { hexazi- } \\
\text { none B } \\
\text { (ng/L) } \\
\text { (68566) }\end{array}$ & $\begin{array}{l}\text { Metola- } \\
\text { chlor } \\
\text { sulfonic } \\
\text { acid } \\
\text { (ng/L) } \\
\text { (68651) }\end{array}$ & $\begin{array}{l}\text { Metola- } \\
\text { chlor } \\
\text { oxanilic } \\
\text { acid } \\
\text { (ng/L) } \\
(68650)\end{array}$ & $\begin{array}{l}\text { Dechlo- } \\
\text { rometola- } \\
\text { chlor } \\
\text { (ng/L) } \\
\text { (68562) }\end{array}$ & $\begin{array}{l}\text { 2-Hydroxy-4-iso- } \\
\text { propylamino- } \\
\text { 6-ethylamino-s- } \\
\text { triazine } \\
\text { (OIET) } \\
\text { (ng/L) } \\
\text { (68660) }\end{array}$ & $\begin{array}{c}\text { Hydroxy- } \\
\text { metola- } \\
\text { chlor } \\
\text { (ng/L) } \\
\text { (68622) }\end{array}$ & & & \\
\hline Benchmark type & HНBР & na & na & na & na & na & na & na & HHBP & na & na & - & - \\
\hline Benchmark level (ng/L) & 126,000 & na & na & na & na & na & na & na & 70,000 & na & na & - & - \\
\hline [LRL] (ng/L) & {$[65]^{1}$} & {$[50]^{1}$} & {$[4.1]^{1}$} & [11] & [3] & [68] & [149] & {$[5]^{1}$} & [8] & {$[5]^{1}$} & {$[100]^{1}$} & - & - \\
\hline \multicolumn{14}{|c|}{ Madera/Chowchilla-Kings Shallow Aquifer study unit (76 wells sampled)²_-Continued } \\
\hline \multicolumn{14}{|c|}{ Kings study area (49 wells sampled) —Continued } \\
\hline S3-MACK-K27 & 996 & 109 & - & - & - & - & - & - & - & - & - & 2 & - \\
\hline S3-MACK-K28 & 62 & - & 26.6 & - & - & - & - & - & - & - & - & 2 & - \\
\hline S3-MACK-K29 & 175 & - & - & - & - & - & - & - & - & - & - & 1 & - \\
\hline S3-MACK-K30 & 80.9 & - & - & - & - & - & - & - & - & - & - & 1 & - \\
\hline S3-MACK-K32 & 68.1 & 115 & 10 & - & - & - & - & - & - & - & - & 3 & - \\
\hline S3-MACK-K33 & 124 & 69.6 & 66.7 & - & - & - & - & - & - & - & - & 3 & - \\
\hline S3-MACK-K35 & - & 42.6 & - & - & - & - & - & - & - & - & - & 1 & - \\
\hline S3-MACK-K37 & - & 40.6 & 2.4 & - & - & - & - & - & - & - & - & 2 & - \\
\hline S3-MACK-K38 & E355 & 195 & 14.6 & - & - & - & - & - & - & - & - & 3 & - \\
\hline S3-MACK-K39 & 208 & 30.5 & - & - & - & - & - & - & - & - & - & 2 & - \\
\hline S3-MACK-K40 & 190 & 25.5 & - & - & - & - & - & - & - & - & - & 2 & - \\
\hline S3-MACK-K41 & 419 & - & 27.2 & - & - & - & - & - & - & - & - & 2 & - \\
\hline S3-MACK-K43 & 8,740 & 793 & - & 5.7 & - & - & - & - & - & - & - & 3 & - \\
\hline S3-MACK-K44 & 11,200 & 626 & 18 & - & - & - & - & - & - & - & - & 3 & - \\
\hline
\end{tabular}


Table 6B. Pesticide degradates detected in samples collected for the Madera/Chowchilla-Kings Shallow Aquifer study unit, Groundwater Ambient Monitoring and Assessment (GAMA) Priority Basin Project, California, August 2013 to April 2014.-Continued

[The five-digit USGS parameter code below the constituent name is used to uniquely identify a specific constituent or property. Samples from 76 grid wells were analyzed, but only samples with detections are listed. All constituents are listed in table 3B. GAMA well identification number: S3-MACK-K, Madera/Chowchilla-Kings Shallow Aquifer Kings study area grid well; S3-MACK-M, Madera/Chowchilla-

Kings Shallow Aquifer Madera/Chowchilla study area grid well. Benchmark type, benchmark level, and LRL as of April 30, 2014. Benchmark levels are reported in the same units as the sample results. Benchmark type: HHBP, U.S. Environmental Protection Agency (EPA) Human Health Benchmarks for Pesticides. Abbreviations: E, estimated or having a higher degree of uncertainty; LRL, laboratory reporting level; na, not available; ng/L, nanograms per liter; USGS, U.S. Geological Survey; —, not detected]

\begin{tabular}{|c|c|c|c|c|c|c|c|c|c|c|c|c|c|}
\hline \multirow{2}{*}{$\begin{array}{c}\text { Primary use or source } \\
\text { GAMA well } \\
\text { identification number }\end{array}$} & \multicolumn{10}{|c|}{ Herbicide degradates } & \multirow{2}{*}{$\begin{array}{c}\begin{array}{c}\text { Fungicide } \\
\text { degra- } \\
\text { date }\end{array} \\
\text { 4-Hy- } \\
\text { droxy- } \\
\text { chloro- } \\
\text { thalonil } \\
\text { (ng/L) } \\
\text { (68336) }\end{array}$} & \multirow[b]{2}{*}{$\begin{array}{c}\text { Detec- } \\
\text { tions } \\
\text { per } \\
\text { well }\end{array}$} & \multirow[b]{2}{*}{$\begin{array}{l}\text { Detection } \\
\text { summary }\end{array}$} \\
\hline & $\begin{array}{c}\text { Chloro- } \\
\text { diamino- } \\
\text { s-triazine } \\
\text { (CAAT) } \\
\text { (ng/L) } \\
\text { (68547) }\end{array}$ & $\begin{array}{l}\text { 2-Chloro- } \\
\text { 6-ethylamino- } \\
\text { 4-amino-s- } \\
\text { triazine } \\
\text { (CEAT) } \\
\text { (ng/L) } \\
\text { (68550) }\end{array}$ & $\begin{array}{l}\text { Demethyl } \\
\text { norflura- } \\
\text { zon } \\
\text { (ng/L) } \\
\text { (68567) }\end{array}$ & $\begin{array}{l}\text { 2-Chloro-4-iso- } \\
\text { propylamino- } \\
\text { 6-amino-s- } \\
\text { triazine } \\
\text { (CIAT) } \\
\text { (ng/L) } \\
(68552)\end{array}$ & $\begin{array}{c}\text { Demethyl } \\
\text { hexazi- } \\
\text { none B } \\
\text { (ng/L) } \\
\text { (68566) }\end{array}$ & $\begin{array}{l}\text { Metola- } \\
\text { chlor } \\
\text { sulfonic } \\
\text { acid } \\
\text { (ng/L) } \\
\text { (68651) }\end{array}$ & $\begin{array}{l}\text { Metola- } \\
\text { chlor } \\
\text { oxanilic } \\
\text { acid } \\
\text { (ng/L) } \\
(68650)\end{array}$ & $\begin{array}{c}\text { Dechlo- } \\
\text { rometola- } \\
\text { chlor } \\
\text { (ng/L) } \\
\text { (68562) }\end{array}$ & $\begin{array}{l}\text { 2-Hydroxy-4-iso- } \\
\text { propylamino- } \\
\text { 6-ethylamino-s- } \\
\text { triazine } \\
\text { (OIET) } \\
\text { (ng/L) } \\
\text { (68660) }\end{array}$ & $\begin{array}{c}\text { Hydroxy- } \\
\text { metola- } \\
\text { chlor } \\
\text { (ng/L) } \\
\text { (68622) }\end{array}$ & & & \\
\hline Benchmark type & HHBP & na & na & na & na & na & na & na & HHBP & na & na & - & - \\
\hline Benchmark level (ng/L) & 126,000 & na & na & na & na & na & na & na & 70,000 & na & na & - & - \\
\hline [LRL] (ng/L) & {$[65]^{1}$} & {$[50]^{1}$} & {$[4.1]^{1}$} & [11] & [3] & [68] & [149] & {$[5]^{1}$} & [8] & {$[5]^{1}$} & {$[100]^{1}$} & - & - \\
\hline \multicolumn{14}{|c|}{ Madera/Chowchilla-Kings Shallow Aquifer study unit (76 wells sampled)²_-Continued } \\
\hline \multicolumn{14}{|c|}{ Kings study area (49 wells sampled) —Continued } \\
\hline S3-MACK-K45 & 5,080 & 1,020 & - & 9.1 & - & - & - & - & - & - & - & 3 & - \\
\hline S3-MACK-K47 & 3,810 & 655 & - & - & - & - & - & - & - & - & - & 2 & - \\
\hline S3-MACK-K48 & - & - & - & 11.1 & - & - & - & - & - & - & - & 1 & - \\
\hline S3-MACK-K49 & 117 & - & - & - & - & - & - & - & - & - & 64.1 & 2 & - \\
\hline Number of wells with detections & 23 & 15 & 11 & 3 & 4 & 3 & 2 & 1 & 1 & 1 & 1 & - & 30 \\
\hline Detection frequency (percent) & 47 & 31 & 22 & 6 & 8 & 6 & 4 & 2 & 2 & 2 & 2 & - & 61 \\
\hline Total detections (number) & - & - & - & - & - & - & - & - & - & - & - & - & 65 \\
\hline
\end{tabular}

${ }^{1}$ The LRL for some constituents changed during the study. The highest LRL was used. The LRL is two times the long-term method detection level (LT-MDL), and detections at concentrations below the LTMDL are not reported and are not counted as detections for the purpose of calculating detection frequencies.

${ }^{2}$ Results are not available for one sample in the Madera/Chowchilla study area: S3-MACK-20 (see appendix). 
Table 7. Results for analyses of trace elements in groundwater samples collected for the Madera/Chowchilla-Kings Shallow Aquifer study unit, Groundwater Ambient Monitoring and Assessment (GAMA) Priority Basin Project, California, August 2013 to April 2014.

[The five-digit USGS parameter code below the constituent name is used to uniquely identify a specific constituent or property. Samples from all 77 grid wells were analyzed. Constituents for which all samples were non-detections (thallium) or are reviewed and rejected (cobalt) are not listed. Information about the constituents given in table 3C. GAMA well identification numbers: S3-MACK-K, Madera/ Chowchilla-Kings Shallow Aquifer Kings study-area grid well; S3-MACK-M, Madera/Chowchilla-Kings Shallow Aquifer Madera/Chowchilla study area grid well. Benchmark type, benchmark level, and LT-MDL as of April 30, 2014. Benchmark types: Maximum contaminant level benchmarks are listed as MCL-US when the MCL-US and MCL-CA are identical, and as MCL-CA when the MCL-CA is lowe than the MCL-US or no MCL-US exists. AL-US, U.S. Environmental Protection Agency (EPA) action level; HBSL, USGS health-based screening level; MCL-CA, State of California maximum contaminant level; MCL-US, EPA maximum contaminant level; NL-CA, State of California notification level; SMCL-CA, State of California secondary maximum contaminant level. Abbreviations: LT-MDL, long-term method detection level; na, not available; SRL, study reporting level; USGS, U.S. Geological Survey; $\mu \mathrm{g} / \mathrm{L}$, micrograms per liter; —, not detected; $\leq$, less than or equal to; *, concentration greater than the benchmark level]

\begin{tabular}{|c|c|c|c|c|c|c|c|c|c|c|c|}
\hline $\begin{array}{c}\text { GAMA } \\
\text { identification } \\
\text { number }\end{array}$ & $\begin{array}{c}\text { Aluminum } \\
(\mu \mathrm{g} / \mathrm{L}) \\
(01106)\end{array}$ & $\begin{array}{c}\text { Antimony } \\
(\mu \mathrm{g} / \mathrm{L}) \\
(01095)\end{array}$ & $\begin{array}{c}\text { Arsenic } \\
(\mu \mathrm{g} / \mathrm{L}) \\
(01000)\end{array}$ & $\begin{array}{c}\text { Barium } \\
(\mu \mathrm{g} / \mathrm{L}) \\
(01005)\end{array}$ & $\begin{array}{c}\text { Beryllium } \\
\text { ( } \mu \mathrm{g} / \mathrm{L}) \\
(01010)\end{array}$ & $\begin{array}{c}\text { Boron } \\
\text { ( } \mu \mathrm{g} / \mathrm{L}) \\
(01020)\end{array}$ & $\begin{array}{c}\text { Cadmium } \\
(\mu \mathrm{g} / \mathrm{L}) \\
(01025)\end{array}$ & $\begin{array}{c}\text { Chromium } \\
(\mu \mathrm{g} / \mathrm{L}) \\
(01030)\end{array}$ & $\begin{array}{c}\text { Copper } \\
\text { ( } \mu g / \mathrm{L}) \\
(01040)\end{array}$ & $\begin{array}{c}\text { Iron } \\
\text { ( } \mu g / L) \\
(01046)\end{array}$ & $\begin{array}{c}\text { Lead } \\
(\mu \mathrm{g} / \mathrm{L}) \\
(01049)\end{array}$ \\
\hline Benchmark type & MCL-CA & MCL-US & MCL-US & MCL-CA & MCL-US & HBSL & MCL-US & MCL-CA & AL-US & SMCL-CA & AL-US \\
\hline Benchmark level & 1,000 & 6 & 10 & 1,000 & 4 & 6,000 & 5 & 50 & 1,300 & 300 & 15 \\
\hline [LT-MDL or SRL] & [2.2] & {$[0.027]$} & {$[0.10]^{1}$} & {$[0.25]^{1}$} & {$[0.02]^{1}$} & {$[5.0]^{1}$} & {$[0.03]^{1}$} & {$[0.3]^{1}$} & {$[2.1]^{2}$} & {$[6]^{2}$} & {$[0.82]^{1,2}$} \\
\hline
\end{tabular}

Madera/Chowchilla-Kings Shallow Aquifer study unit (77 wells sampled)

Madera/Chowchilla study area (28 wells sampled)

\begin{tabular}{|c|c|c|c|c|c|c|c|c|c|c|c|}
\hline S3-MACK-M01 & 5.0 & 0.034 & $* 16.6$ & 13.5 & - & 19 & - & - & - & 23.8 & - \\
\hline S3-MACK-M02 & 4.7 & 0.062 & 5.5 & 49.4 & - & 26 & - & 3.1 & - & 15.0 & $\leq 0.053$ \\
\hline S3-MACK-M03 & 6.5 & 0.033 & 1.5 & 676 & - & 94 & - & 1.3 & 2.8 & 10.7 & $\leq 0.133$ \\
\hline S3-MACK-M04 & 4.7 & 0.060 & 3.8 & 203 & - & 32 & - & 3.5 & $\leq 2.0$ & - & $\leq 0.283$ \\
\hline S3-MACK-M05 & 3.5 & 0.056 & 2.4 & 390 & - & 18 & - & 2.8 & $\leq 0.8$ & - & $\leq 0.206$ \\
\hline S3-MACK-M06 & 3.1 & 0.053 & 3.8 & 455 & - & 26 & - & 4.6 & $\leq 1.4$ & - & $\leq 0.424$ \\
\hline S3-MACK-M07 & 4.3 & 0.076 & 3.9 & 164 & - & 20 & - & 0.9 & 3.9 & - & 1.69 \\
\hline S3-MACK-M08 & 3.4 & 0.040 & 1.1 & 355 & - & 19 & - & 2.5 & 2.4 & - & $\leq 0.483$ \\
\hline S3-MACK-M09 & 3.9 & - & 1.1 & 287 & - & 20 & - & 2.3 & $\leq 1.9$ & - & $\leq 0.125$ \\
\hline S3-MACK-M10 & 3.6 & 0.037 & 1.2 & 398 & - & 23 & - & 3.0 & $\leq 1.1$ & 7.5 & $\leq 0.238$ \\
\hline S3-MACK-M11 & 3.0 & 0.071 & 1.4 & 95.7 & - & 36 & - & 1.4 & $\leq 1.7$ & $\leq 5.0$ & $\leq 0.168$ \\
\hline S3-MACK-M12 & 5.7 & 0.078 & 2.5 & 56.3 & - & 23 & - & 2.3 & $\leq 1.1$ & - & $\leq 0.109$ \\
\hline S3-MACK-M13 & 2.6 & 0.031 & 1.3 & 92.7 & - & 27 & - & 1.7 & $\leq 0.94$ & - & $\leq 0.135$ \\
\hline S3-MACK-M14 & 10.8 & - & 1.0 & 104 & - & 17 & - & 1.8 & 2.4 & 12.5 & $\leq 0.161$ \\
\hline S3-MACK-M15 & 4.1 & - & 1.6 & 144 & - & 19 & - & 2.5 & $\leq 1.3$ & - & $\leq 0.521$ \\
\hline S3-MACK-M16 & 4.4 & 0.040 & 1.2 & 505 & - & 44 & - & 2.9 & 3.8 & - & $\leq 0.491$ \\
\hline S3-MACK-M17 & 4.9 & 0.047 & 1.7 & 721 & - & 22 & - & 4.0 & $\leq 1.4$ & - & $\leq 0.807$ \\
\hline S3-MACK-M18 & 2.9 & - & 1.4 & 184 & - & 18 & - & 4.7 & $\leq 2.0$ & 6.1 & $\leq 0.313$ \\
\hline S3-MACK-M19 & 4.8 & 0.031 & 1.8 & 154 & - & 18 & - & 1.8 & 6.2 & - & $\leq 0.451$ \\
\hline
\end{tabular}


Table 7. Results for analyses of trace elements in groundwater samples collected for the Madera/Chowchilla-Kings Shallow Aquifer study unit, Groundwater Ambient Monitoring and Assessment (GAMA) Priority Basin Project, California, August 2013 to April 2014.-Continued

[The five-digit USGS parameter code below the constituent name is used to uniquely identify a specific constituent or property. Samples from all 77 grid wells were analyzed. Constituents for which all samples were non-detections (thallium) or are reviewed and rejected (cobalt) are not listed. Information about the constituents given in table 3C. GAMA well identification numbers: S3-MACK-K, Madera/ Chowchilla-Kings Shallow Aquifer Kings study-area grid well; S3-MACK-M, Madera/Chowchilla-Kings Shallow Aquifer Madera/Chowchilla study area grid well. Benchmark type, benchmark level, and LT-MDL as of April 30, 2014. Benchmark types: Maximum contaminant level benchmarks are listed as MCL-US when the MCL-US and MCL-CA are identical, and as MCL-CA when the MCL-CA is lower than the MCL-US or no MCL-US exists. AL-US, U.S. Environmental Protection Agency (EPA) action level; HBSL, USGS health-based screening level; MCL-CA, State of California maximum contaminant level; MCL-US, EPA maximum contaminant level; NL-CA, State of California notification level; SMCL-CA, State of California secondary maximum contaminant level. Abbreviations: LT-MDL, long-term method detection level; na, not available; SRL, study reporting level; USGS, U.S. Geological Survey; $\mu \mathrm{g} / \mathrm{L}$, micrograms per liter; - , not detected; $\leq$, less than or equal to; *, concentration greater than the benchmark level]

\begin{tabular}{|c|c|c|c|c|c|c|c|c|c|c|c|}
\hline $\begin{array}{c}\text { GAMA } \\
\text { identification } \\
\text { number }\end{array}$ & $\begin{array}{c}\text { Aluminum } \\
(\mu \mathrm{g} / \mathrm{L}) \\
(01106) \\
\end{array}$ & $\begin{array}{c}\text { Antimony } \\
(\mu \mathrm{g} / \mathrm{L}) \\
(01095) \\
\end{array}$ & $\begin{array}{c}\text { Arsenic } \\
(\mu \mathrm{g} / \mathrm{L}) \\
(01000)\end{array}$ & $\begin{array}{c}\text { Barium } \\
(\mu \mathrm{g} / \mathrm{L}) \\
(01005)\end{array}$ & $\begin{array}{c}\text { Beryllium } \\
(\mu \mathrm{g} / \mathrm{L}) \\
(01010) \\
\end{array}$ & $\begin{array}{c}\text { Boron } \\
(\mu \mathrm{g} / \mathrm{L}) \\
(01020)\end{array}$ & $\begin{array}{c}\text { Cadmium } \\
\text { ( } \mu \mathrm{g} / \mathrm{L}) \\
(01025) \\
\end{array}$ & $\begin{array}{c}\text { Chromium } \\
(\mu \mathrm{g} / \mathrm{L}) \\
(01030) \\
\end{array}$ & $\begin{array}{c}\text { Copper } \\
(\mu \mathrm{g} / \mathrm{L}) \\
(01040) \\
\end{array}$ & $\begin{array}{c}\text { Iron } \\
(\mu \mathrm{g} / \mathrm{L}) \\
(01046) \\
\end{array}$ & $\begin{array}{c}\begin{array}{c}\text { Lead } \\
(\mu \mathrm{g} / \mathrm{L}) \\
(01049)\end{array} \\
\end{array}$ \\
\hline Benchmark type & MCL-CA & MCL-US & MCL-US & MCL-CA & MCL-US & HBSL & MCL-US & MCL-CA & AL-US & SMCL-CA & AL-US \\
\hline Benchmark level & 1,000 & 6 & 10 & 1,000 & 4 & 6,000 & 5 & 50 & 1,300 & 300 & 15 \\
\hline [LT-MDL or SRL] & [2.2] & {$[0.027]$} & {$[0.10]^{1}$} & {$[0.25]^{1}$} & {$[0.02]^{1}$} & {$[5.0]^{1}$} & {$[0.03]^{1}$} & {$[0.3]^{1}$} & {$[2.1]^{2}$} & {$[6]^{2}$} & {$[0.82]^{1,2}$} \\
\hline \multicolumn{12}{|c|}{ Madera/Chowchilla-Kings Shallow Aquifer study unit (77 wells sampled)_Continued } \\
\hline \multicolumn{12}{|c|}{ Madera/Chowchilla study area (28 wells sampled)_-Continued } \\
\hline S3-MACK-M20 & 3.2 & - & 1.1 & 52.2 & - & 22 & - & 2.5 & $\leq 0.99$ & - & $\leq 0.319$ \\
\hline S3-MACK-M21 & 4.0 & - & 0.64 & 128 & - & 21 & - & 0.89 & 2.7 & 23.9 & $\leq 0.280$ \\
\hline S3-MACK-M22 & 2.6 & - & 1.1 & 105 & - & 17 & - & 3.4 & $\leq 1.9$ & - & $\leq 0.364$ \\
\hline S3-MACK-M23 & 2.3 & 0.080 & 3.7 & 14.8 & - & 20 & - & - & - & 10.5 & $\leq 0.257$ \\
\hline S3-MACK-M24 & 8.3 & 0.048 & 2.7 & 54.6 & - & 13 & - & 1.6 & - & $\leq 4.7$ & $\leq 0.057$ \\
\hline S3-MACK-M25 & 5.3 & 0.033 & 2.8 & 94.9 & - & 21 & - & 2.2 & - & 9.1 & $\leq 0.053$ \\
\hline S3-MACK-M26 & 4.7 & 0.036 & 2.9 & 74.3 & - & 56 & - & 2.8 & - & 26.7 & $\leq 0.448$ \\
\hline S3-MACK-M27 & 4.0 & 0.032 & 1.8 & 85.0 & - & 28 & - & 1.0 & $\leq 1.8$ & 10.1 & $\leq 0.200$ \\
\hline S3-MACK-M28 & 4.1 & - & 3.0 & 51.6 & - & 32 & - & - & - & 86.3 & - \\
\hline \multicolumn{12}{|c|}{ Kings study area (49 wells sampled) } \\
\hline S3-MACK-K01 & - & 0.174 & 2.2 & 68.3 & - & 491 & 0.239 & - & - & $* 2,190$ & $\leq 0.738$ \\
\hline S3-MACK-K02 & 3.7 & 0.045 & $* 23.0$ & 70.4 & 0.034 & 700 & - & - & - & 42.3 & $\leq 0.231$ \\
\hline S3-MACK-K03 & 3.3 & 0.039 & 2.1 & 58.8 & - & 22 & - & - & - & - & $\leq 0.388$ \\
\hline S3-MACK-K04 & 3.2 & - & 1.5 & 133 & - & 159 & - & - & - & $* 489$ & $\leq 0.067$ \\
\hline S3-MACK-K05 & 3.0 & 0.030 & 3.6 & 76.6 & - & 258 & - & - & - & 136 & $\leq 0.106$ \\
\hline S3-MACK-K06 & 4.2 & - & 2.3 & 28.2 & - & 441 & - & - & - & * 682 & - \\
\hline S3-MACK-K07 & 2.4 & 0.035 & 9.0 & 78.1 & 0.087 & 1,460 & 0.037 & - & - & 65.8 & $\leq 0.063$ \\
\hline S3-MACK-K08 & 5.1 & 0.135 & 2.0 & 153 & - & 154 & 0.047 & 4.4 & 4.5 & 21.3 & $\leq 0.349$ \\
\hline S3-MACK-K09 & 4.8 & - & $* 17.7$ & 32.2 & - & 158 & - & - & - & 9.4 & - \\
\hline S3-MACK-K10 & - & 0.060 & 2.7 & 163 & - & 37 & 0.097 & 9.0 & - & 50.8 & $\leq 0.118$ \\
\hline
\end{tabular}


Table 7. Results for analyses of trace elements in groundwater samples collected for the Madera/Chowchilla-Kings Shallow Aquifer study unit, Groundwater Ambient Monitoring and Assessment (GAMA) Priority Basin Project, California, August 2013 to April 2014._Continued

[The five-digit USGS parameter code below the constituent name is used to uniquely identify a specific constituent or property. Samples from all 77 grid wells were analyzed. Constituents for which all samples were non-detections (thallium) or are reviewed and rejected (cobalt) are not listed. Information about the constituents given in table 3C. GAMA well identification numbers: S3-MACK-K, Madera/ Chowchilla-Kings Shallow Aquifer Kings study-area grid well; S3-MACK-M, Madera/Chowchilla-Kings Shallow Aquifer Madera/Chowchilla study area grid well. Benchmark type, benchmark level, and LT-MDL as of April 30, 2014. Benchmark types: Maximum contaminant level benchmarks are listed as MCL-US when the MCL-US and MCL-CA are identical, and as MCL-CA when the MCL-CA is lowe than the MCL-US or no MCL-US exists. AL-US, U.S. Environmental Protection Agency (EPA) action level; HBSL, USGS health-based screening level; MCL-CA, State of California maximum contaminant level; MCL-US, EPA maximum contaminant level; NL-CA, State of California notification level; SMCL-CA, State of California secondary maximum contaminant level. Abbreviations: LT-MDL, long-term method detection level; na, not available; SRL, study reporting level; USGS, U.S. Geological Survey; $\mu \mathrm{g} / \mathrm{L}$, micrograms per liter; —, not detected; $\leq$, less than or equal to; *, concentration greater than the benchmark level]

\begin{tabular}{|c|c|c|c|c|c|c|c|c|c|c|c|}
\hline $\begin{array}{c}\text { GAMA } \\
\text { identification } \\
\text { number }\end{array}$ & $\begin{array}{c}\text { Aluminum } \\
(\mu \mathrm{g} / \mathrm{L}) \\
(01106)\end{array}$ & $\begin{array}{c}\text { Antimony } \\
(\mu \mathrm{g} / \mathrm{L}) \\
(01095)\end{array}$ & $\begin{array}{c}\text { Arsenic } \\
(\mu \mathrm{g} / \mathrm{L}) \\
(01000)\end{array}$ & $\begin{array}{c}\text { Barium } \\
(\mu \mathrm{g} / \mathrm{L}) \\
(01005)\end{array}$ & $\begin{array}{c}\text { Beryllium } \\
(\mu \mathrm{g} / \mathrm{L}) \\
(01010)\end{array}$ & $\begin{array}{c}\text { Boron } \\
(\mu g / L) \\
(01020)\end{array}$ & $\begin{array}{c}\text { Cadmium } \\
\text { ( } \mu \mathrm{g} / \mathrm{L}) \\
(01025)\end{array}$ & $\begin{array}{c}\text { Chromium } \\
(\mu \mathrm{g} / \mathrm{L}) \\
(01030)\end{array}$ & $\begin{array}{c}\text { Copper } \\
\text { ( } \mu \mathrm{gg} / \mathrm{L}) \\
(01040)\end{array}$ & $\begin{array}{c}\text { Iron } \\
(\mu \mathrm{g} / \mathrm{L}) \\
(01046)\end{array}$ & $\begin{array}{c}\text { Lead } \\
(\mu \mathrm{g} / \mathrm{L}) \\
(01049)\end{array}$ \\
\hline Benchmark type & MCL-CA & MCL-US & MCL-US & MCL-CA & MCL-US & HBSL & MCL-US & MCL-CA & AL-US & SMCL-CA & AL-US \\
\hline Benchmark level & 1,000 & 6 & 10 & 1,000 & 4 & 6,000 & 5 & 50 & 1,300 & 300 & 15 \\
\hline [LT-MDL or SRL] & [2.2] & {$[0.027]$} & {$[0.10]^{1}$} & {$[0.25]^{1}$} & {$[0.02]^{1}$} & {$[5.0]^{1}$} & {$[0.03]^{1}$} & {$[0.3]^{1}$} & {$[2.1]^{2}$} & {$[6]^{2}$} & {$[0.82]^{1,2}$} \\
\hline
\end{tabular}

Madera/Chowchilla-Kings Shallow Aquifer study unit (77 wells sampled)_Continued

Kings study area (49 wells sampled) - Continued

\begin{tabular}{|c|c|c|c|c|c|c|c|c|c|c|c|}
\hline S3-MACK-K11 & 3.8 & 0.065 & 2.9 & 65.5 & - & 41 & - & 2.8 & - & $\leq 5.7$ & $\leq 0.332$ \\
\hline S3-MACK-K12 & 2.9 & 0.049 & 4.7 & 42.6 & - & 300 & 0.033 & - & $\leq 0.82$ & - & $\leq 0.251$ \\
\hline S3-MACK-K13 & 16.7 & - & * 31.8 & 27.7 & 0.043 & 795 & 0.061 & - & - & 20.7 & $\leq 0.043$ \\
\hline S3-MACK-K14 & 8.1 & - & $* 34.0$ & 6.72 & - & 136 & - & - & - & 11.2 & $\leq 0.051$ \\
\hline S3-MACK-K15 & 4.3 & 0.064 & 0.77 & 7.97 & - & 20 & - & 1.2 & - & - & $\leq 0.134$ \\
\hline S3-MACK-K16 & 2.2 & 0.048 & 0.42 & 182 & - & 100 & - & 2.8 & 2.4 & $\leq 5.9$ & 1.05 \\
\hline S3-MACK-K17 & 3.7 & 0.060 & 2.2 & 4.87 & - & 10 & - & 0.32 & - & - & $\leq 0.065$ \\
\hline S3-MACK-K18 & 3.5 & 0.069 & 2.1 & 148 & - & 99 & - & 4.4 & $\leq 0.99$ & $\leq 4.5$ & $\leq 0.237$ \\
\hline S3-MACK-K19 & 3.6 & 0.077 & 3.0 & 123 & - & 63 & - & 5.1 & 3.6 & - & $\leq 0.586$ \\
\hline S3-MACK-K20 & 4.7 & 0.066 & 2.8 & 77.7 & - & 59 & - & 4.0 & 3.2 & - & $\leq 0.143$ \\
\hline S3-MACK-K21 & 4.2 & 0.097 & 4.0 & 20.1 & - & 75 & 0.033 & 0.64 & $\leq 0.93$ & - & $\leq 0.285$ \\
\hline S3-MACK-K22 & 5.2 & 0.077 & 2.8 & 41.6 & - & 19 & - & 2.5 & 5.7 & - & $\leq 0.396$ \\
\hline S3-MACK-K23 & 5.3 & 0.089 & 2.3 & 12.0 & - & 33 & - & 0.92 & - & - & - \\
\hline S3-MACK-K24 & 4.5 & 0.066 & 3.8 & 120 & - & 56 & - & 3.0 & - & - & $\leq 0.082$ \\
\hline S3-MACK-K25 & - & 0.062 & 1.2 & 24.3 & - & 24 & - & 1.0 & $\leq 1.3$ & - & $\leq 0.255$ \\
\hline S3-MACK-K26 & 2.7 & 0.045 & 1.1 & 11.2 & - & 11 & - & 0.37 & $\leq 1.6$ & - & $\leq 0.143$ \\
\hline S3-MACK-K27 & 3.7 & 0.035 & 0.92 & 160 & - & 43 & - & 2.8 & $\leq 1.3$ & - & $\leq 0.470$ \\
\hline S3-MACK-K28 & 3.5 & 0.047 & 1.4 & 54.2 & - & 23 & - & 0.71 & - & - & $\leq 0.107$ \\
\hline S3-MACK-K29 & 3.1 & 0.049 & 2.5 & 120 & - & 26 & - & 1.6 & $\leq 1.0$ & 9.1 & $\leq 0.149$ \\
\hline
\end{tabular}


Table 7. Results for analyses of trace elements in groundwater samples collected for the Madera/Chowchilla-Kings Shallow Aquifer study unit, Groundwater Ambient Monitoring and Assessment (GAMA) Priority Basin Project, California, August 2013 to April 2014.-Continued

[The five-digit USGS parameter code below the constituent name is used to uniquely identify a specific constituent or property. Samples from all 77 grid wells were analyzed. Constituents for which all samples were non-detections (thallium) or are reviewed and rejected (cobalt) are not listed. Information about the constituents given in table 3C. GAMA well identification numbers: S3-MACK-K, Madera/ Chowchilla-Kings Shallow Aquifer Kings study-area grid well; S3-MACK-M, Madera/Chowchilla-Kings Shallow Aquifer Madera/Chowchilla study area grid well. Benchmark type, benchmark level, and LT-MDL as of April 30, 2014. Benchmark types: Maximum contaminant level benchmarks are listed as MCL-US when the MCL-US and MCL-CA are identical, and as MCL-CA when the MCL-CA is lower than the MCL-US or no MCL-US exists. AL-US, U.S. Environmental Protection Agency (EPA) action level; HBSL, USGS health-based screening level; MCL-CA, State of California maximum contaminant level; MCL-US, EPA maximum contaminant level; NL-CA, State of California notification level; SMCL-CA, State of California secondary maximum contaminant level. Abbreviations: LT-MDL, long-term method detection level; na, not available; SRL, study reporting level; USGS, U.S. Geological Survey; $\mu \mathrm{g} / \mathrm{L}$, micrograms per liter; - , not detected; $\leq$, less than or equal to; *, concentration greater than the benchmark level]

\begin{tabular}{|c|c|c|c|c|c|c|c|c|c|c|c|}
\hline $\begin{array}{c}\text { GAMA } \\
\text { identification } \\
\text { number }\end{array}$ & $\begin{array}{c}\text { Aluminum } \\
(\mu \mathrm{g} / \mathrm{L}) \\
(01106)\end{array}$ & $\begin{array}{c}\text { Antimony } \\
(\mu \mathrm{g} / \mathrm{L}) \\
(01095)\end{array}$ & $\begin{array}{c}\text { Arsenic } \\
(\mu \mathrm{g} / \mathrm{L}) \\
(01000)\end{array}$ & $\begin{array}{c}\text { Barium } \\
\text { ( } \mu \mathrm{g} / \mathrm{L}) \\
(01005)\end{array}$ & $\begin{array}{c}\text { Beryllium } \\
(\mu \mathrm{g} / \mathrm{L}) \\
(01010)\end{array}$ & $\begin{array}{c}\text { Boron } \\
(\mu \mathrm{g} / \mathrm{L}) \\
(01020)\end{array}$ & $\begin{array}{c}\text { Cadmium } \\
\text { ( } \mu \mathrm{g} / \mathrm{L}) \\
(01025)\end{array}$ & $\begin{array}{c}\text { Chromium } \\
(\mu \mathrm{g} / \mathrm{L}) \\
(01030)\end{array}$ & $\begin{array}{c}\text { Copper } \\
\text { ( } \mu \mathrm{g} / \mathrm{L}) \\
(01040)\end{array}$ & $\begin{array}{c}\text { Iron } \\
(\mu \mathrm{g} / \mathrm{L}) \\
(01046)\end{array}$ & $\begin{array}{c}\text { Lead } \\
(\mu \mathrm{g} / \mathrm{L}) \\
(01049)\end{array}$ \\
\hline Benchmark type & MCL-CA & MCL-US & MCL-US & MCL-CA & MCL-US & HBSL & MCL-US & MCL-CA & AL-US & SMCL-CA & AL-US \\
\hline Benchmark level & 1,000 & 6 & 10 & 1,000 & 4 & 6,000 & 5 & 50 & 1,300 & 300 & 15 \\
\hline [LT-MDL or SRL] & {$[2.2]$} & {$[0.027]$} & {$[0.10]^{1}$} & {$[0.25]^{1}$} & {$[0.02]^{1}$} & {$[5.0]^{1}$} & {$[0.03]^{1}$} & {$[0.3]^{1}$} & {$[2.1]^{2}$} & {$[6]^{2}$} & {$[0.82]^{1,2}$} \\
\hline
\end{tabular}

Madera/Chowchilla-Kings Shallow Aquifer study unit (77 wells sampled)_Continued

Kings study area (49 wells sampled) - Continued

\begin{tabular}{|c|c|c|c|c|c|c|c|c|c|c|c|}
\hline S3-MACK-K30 & 3.5 & 0.068 & 3.8 & 27.9 & - & 25 & - & 0.77 & $\leq 1.1$ & - & $\leq 0.129$ \\
\hline S3-MACK-K31 & - & 0.060 & 3.8 & 44.4 & - & 21 & - & 2.7 & $\leq 0.86$ & - & $\leq 0.158$ \\
\hline S3-MACK-K32 & 2.8 & 0.039 & 1.5 & 10.7 & - & 13 & - & 0.54 & $\leq 1.7$ & - & $\leq 0.374$ \\
\hline S3-MACK-K33 & - & - & 0.84 & 178 & - & 29 & - & 2.2 & - & - & 0.865 \\
\hline S3-MACK-K34 & 4.9 & 0.059 & 1.4 & 144 & - & 37 & - & 3.6 & $\leq 1.2$ & - & - \\
\hline S3-MACK-K35 & 5.1 & 0.037 & 2.2 & 39.0 & - & 13 & - & 2.0 & - & - & - \\
\hline S3-MACK-K36 & 9.5 & 0.029 & 1.2 & 95.8 & - & 13 & - & 5.9 & - & 19.1 & - \\
\hline S3-MACK-K37 & 4.5 & 0.049 & 1.4 & 42.3 & - & 25 & - & - & - & - & - \\
\hline S3-MACK-K38 & 3.8 & 0.027 & 0.73 & 133 & - & 26 & - & 1.2 & $\leq 1.2$ & - & - \\
\hline S3-MACK-K39 & 2.8 & 0.039 & 0.81 & 162 & - & 13 & - & 1.4 & - & - & $\leq 0.068$ \\
\hline S3-MACK-K40 & - & 0.032 & 1.1 & 186 & - & 35 & - & 1.2 & 4.6 & - & $\leq 0.203$ \\
\hline S3-MACK-K41 & 3.6 & - & 1.1 & 145 & - & 21 & - & 1.1 & $\leq 1.0$ & $\leq 5.6$ & $\leq 0.054$ \\
\hline S3-MACK-K42 & 3.8 & 0.090 & 5.0 & 223 & - & 38 & - & 0.99 & $\leq 1.9$ & - & $\leq 0.180$ \\
\hline S3-MACK-K43 & 4.1 & 0.048 & 2.6 & 170 & - & 27 & - & 0.40 & 2.9 & $\leq 4.7$ & $\leq 0.481$ \\
\hline S3-MACK-K44 & 3.7 & - & 0.83 & 286 & - & 15 & - & 0.38 & $\leq 1.1$ & 7.2 & $\leq 0.067$ \\
\hline S3-MACK-K45 & - & 0.087 & 4.5 & 195 & - & 30 & - & - & $\leq 1.2$ & - & $\leq 0.101$ \\
\hline S3-MACK-K46 & 3.4 & - & 0.24 & 28.7 & - & 13 & - & - & - & 221 & $\leq 0.054$ \\
\hline S3-MACK-K47 & 3.3 & - & 0.6 & 54.4 & - & 19 & - & 0.35 & 2.6 & $\leq 4.4$ & $\leq 0.063$ \\
\hline S3-MACK-K48 & 3.4 & 0.029 & 2.2 & 185 & - & 20 & - & 0.35 & - & - & $\leq 0.046$ \\
\hline S3-MACK-K49 & - & 0.040 & 1.8 & 200 & - & 12 & - & 1.2 & 2.6 & - & $\leq 0.306$ \\
\hline
\end{tabular}


Table 7. Results for analyses of trace elements in groundwater samples collected for the Madera/Chowchilla-Kings Shallow Aquifer study unit, Groundwater Ambient Monitoring and Assessment (GAMA) Priority Basin Project, California, August 2013 to April 2014._Continued

[The five-digit USGS parameter code below the constituent name is used to uniquely identify a specific constituent or property. Samples from all 77 grid wells were analyzed. Constituents for which all samples were non-detections (thallium) or are reviewed and rejected (cobalt) are not listed. Information about the constituents given in table 3C. GAMA well identification numbers: S3-MACK-K, Madera/ Chowchilla-Kings Shallow Aquifer Kings study-area grid well; S3-MACK-M, Madera/Chowchilla-Kings Shallow Aquifer Madera/Chowchilla study area grid well. Benchmark type, benchmark level, and LT-MDL as of April 30, 2014. Benchmark types: Maximum contaminant level benchmarks are listed as MCL-US when the MCL-US and MCL-CA are identical, and as MCL-CA when the MCL-CA is lowe than the MCL-US or no MCL-US exists. AL-US, U.S. Environmental Protection Agency (EPA) action level; HBSL, USGS health-based screening level; MCL-CA, State of California maximum contaminant level; MCL-US, EPA maximum contaminant level; NL-CA, State of California notification level; SMCL-CA, State of California secondary maximum contaminant level. Abbreviations: LT-MDL, long-term method detection level; na, not available; SRL, study reporting level; USGS, U.S. Geological Survey; $\mu \mathrm{g} / \mathrm{L}$, micrograms per liter; —, not detected; $\leq$, less than or equal to; *, concentration greater than the benchmark level]

\begin{tabular}{|c|c|c|c|c|c|c|c|c|c|c|}
\hline $\begin{array}{c}\text { GAMA } \\
\text { identification } \\
\text { number }\end{array}$ & $\begin{array}{l}\text { Lithium } \\
\text { ( } \mu \mathrm{g} / \mathrm{L}) \\
(\mathbf{0 1 1 3 0})\end{array}$ & $\begin{array}{c}\text { Manganese } \\
(\mu \mathrm{g} / \mathrm{L}) \\
(01056)\end{array}$ & $\begin{array}{c}\text { Molybdenum } \\
(\mu \mathrm{g} / \mathrm{L}) \\
(01060)\end{array}$ & $\begin{array}{c}\text { Nickel } \\
\text { (pg/L) } \\
(01065)\end{array}$ & $\begin{array}{c}\text { Selenium } \\
(\mu \mathrm{g} / \mathrm{L}) \\
(01145)\end{array}$ & $\begin{array}{c}\text { Silver } \\
(\mu \mathrm{g} / \mathrm{L}) \\
(01075)\end{array}$ & $\begin{array}{c}\text { Strontium } \\
(\mu \mathrm{g} / \mathrm{L}) \\
(01080)\end{array}$ & $\begin{array}{c}\text { Uranium } \\
\text { ( } \mu \mathrm{g} / \mathrm{L}) \\
(22703)\end{array}$ & $\begin{array}{c}\text { Vanadium } \\
(\mu \mathrm{g} / \mathrm{L}) \\
(01085)\end{array}$ & $\begin{array}{c}\text { Zinc } \\
(\mu \mathrm{g} / \mathrm{L}) \\
(01090)\end{array}$ \\
\hline Benchmark type & na & HBSL & HBSL & MCL-CA & MCL-US & HBSL & HBSL & MCL-US & NL-CA & HBSL \\
\hline Benchmark level & na & 300 & 40 & 100 & 50 & 100 & 4,000 & 30 & 50 & 2,000 \\
\hline [LT-MDL or SRL] & {$[0.22]$} & {$[0.66]^{1,2}$} & {$[0.05]^{1}$} & {$[0.21]^{1,2}$} & {$[0.05]^{1}$} & {$[0.02]^{1}$} & {$[0.8]^{1}$} & {$[0.014]^{1}$} & {$[0.08]$} & {$[6.2]^{1,2}$} \\
\hline
\end{tabular}

Madera/Chowchilla-Kings Shallow Aquifer study unit (77 wells sampled)_Continued

Madera/Chowchilla study area (28 wells sampled)—Continued

\begin{tabular}{|c|c|c|c|c|c|c|c|c|c|c|}
\hline S3-MACK-M01 & 3.76 & 14.85 & 7.64 & - & - & - & 70.0 & 0.122 & 0.75 & $\leq 2.2$ \\
\hline S3-MACK-M02 & 8.04 & - & 2.16 & $\leq 0.20$ & 0.22 & - & 175 & 2.48 & 15.6 & 9.1 \\
\hline S3-MACK-M03 & 4.70 & $\leq 0.53$ & 2.38 & 2.08 & 0.15 & 0.025 & 1,580 & * 59.9 & 22.1 & 28.3 \\
\hline S3-MACK-M04 & 16.4 & - & 1.73 & 0.75 & 0.41 & - & 728 & 28.1 & 20.0 & 34.4 \\
\hline S3-MACK-M05 & 13.5 & - & 1.02 & 0.72 & 0.36 & - & 974 & 22.0 & 14.1 & 22.2 \\
\hline S3-MACK-M06 & 4.35 & - & 0.721 & 0.34 & 0.29 & - & 534 & 4.40 & 19.1 & 12.0 \\
\hline S3-MACK-M07 & 4.19 & - & 1.25 & 0.68 & 0.08 & - & 368 & 3.69 & 21.7 & 26.6 \\
\hline S3-MACK-M08 & 2.34 & $\leq 0.59$ & 0.738 & 0.49 & 0.31 & - & 692 & 7.29 & 18.1 & 8.0 \\
\hline S3-MACK-M09 & 0.94 & - & 0.989 & 0.39 & 0.26 & - & 537 & 3.26 & 35.1 & 97.6 \\
\hline S3-MACK-M10 & 8.22 & - & 0.841 & 2.34 & 0.42 & - & 849 & 24.3 & 24.4 & 33.4 \\
\hline S3-MACK-M11 & 16.9 & 0.83 & 1.59 & 1.80 & 0.24 & - & 397 & $* 54.6$ & 10.0 & 59.3 \\
\hline S3-MACK-M12 & 22.6 & - & 2.21 & 0.25 & 0.37 & - & 268 & 14.5 & 15.4 & 18.6 \\
\hline S3-MACK-M13 & 1.76 & 7.66 & 2.20 & 1.08 & 0.28 & - & 178 & 0.506 & 23.4 & 20.4 \\
\hline S3-MACK-M14 & 0.77 & 1.39 & 1.41 & 0.64 & 0.23 & - & 217 & 0.568 & 24.8 & 69.2 \\
\hline S3-MACK-M15 & 0.61 & - & 1.36 & 0.66 & 0.18 & - & 257 & 0.746 & 39.9 & 68.0 \\
\hline S3-MACK-M16 & 3.89 & - & 1.05 & 0.56 & 0.05 & - & 840 & 17.2 & 17.7 & 18.7 \\
\hline S3-MACK-M17 & 8.00 & - & 0.439 & 1.02 & 0.52 & - & 1,070 & 16.9 & 16.0 & 113 \\
\hline S3-MACK-M18 & 0.88 & - & 1.09 & $\leq 0.21$ & 0.21 & - & 319 & 0.739 & 19.3 & 31.5 \\
\hline S3-MACK-M19 & 1.24 & 0.99 & 2.88 & 0.38 & 0.20 & - & 229 & 0.179 & 29.1 & 73.1 \\
\hline
\end{tabular}


Table 7. Results for analyses of trace elements in groundwater samples collected for the Madera/Chowchilla-Kings Shallow Aquifer study unit, Groundwater Ambient Monitoring and Assessment (GAMA) Priority Basin Project, California, August 2013 to April 2014.-Continued

[The five-digit USGS parameter code below the constituent name is used to uniquely identify a specific constituent or property. Samples from all 77 grid wells were analyzed. Constituents for which all samples were non-detections (thallium) or are reviewed and rejected (cobalt) are not listed. Information about the constituents given in table 3C. GAMA well identification numbers: S3-MACK-K, Madera/ Chowchilla-Kings Shallow Aquifer Kings study-area grid well; S3-MACK-M, Madera/Chowchilla-Kings Shallow Aquifer Madera/Chowchilla study area grid well. Benchmark type, benchmark level, and LT-MDL as of April 30, 2014. Benchmark types: Maximum contaminant level benchmarks are listed as MCL-US when the MCL-US and MCL-CA are identical, and as MCL-CA when the MCL-CA is lower than the MCL-US or no MCL-US exists. AL-US, U.S. Environmental Protection Agency (EPA) action level; HBSL, USGS health-based screening level; MCL-CA, State of California maximum contaminant level; MCL-US, EPA maximum contaminant level; NL-CA, State of California notification level; SMCL-CA, State of California secondary maximum contaminant level. Abbreviations: LT-MDL, long-term method detection level; na, not available; SRL, study reporting level; USGS, U.S. Geological Survey; $\mu \mathrm{g} / \mathrm{L}$, micrograms per liter; - , not detected; $\leq$, less than or equal to; *, concentration greater than the benchmark level]

\begin{tabular}{|c|c|c|c|c|c|c|c|c|c|c|}
\hline $\begin{array}{c}\text { GAMA } \\
\text { identification } \\
\text { number }\end{array}$ & $\begin{array}{l}\text { Lithium } \\
\text { ( } \mu \mathrm{g} / \mathrm{L}) \\
(01130)\end{array}$ & $\begin{array}{c}\text { Manganese } \\
(\mu \mathrm{g} / \mathrm{L}) \\
(01056)\end{array}$ & $\begin{array}{c}\text { Molybdenum } \\
(\mu \mathrm{g} / \mathrm{L}) \\
(01060)\end{array}$ & $\begin{array}{c}\text { Nickel } \\
\text { (pg/L) } \\
(01065)\end{array}$ & $\begin{array}{c}\text { Selenium } \\
(\mu \mathrm{g} / \mathrm{L}) \\
(01145) \\
\end{array}$ & $\begin{array}{c}\text { Silver } \\
(\mu \mathrm{g} / \mathrm{L}) \\
(01075) \\
\end{array}$ & $\begin{array}{c}\text { Strontium } \\
(\mu \mathrm{g} / \mathrm{L}) \\
(01080) \\
\end{array}$ & $\begin{array}{c}\text { Uranium } \\
\text { ( } \mu \mathrm{g} / \mathrm{L}) \\
(22703)\end{array}$ & $\begin{array}{c}\text { Vanadium } \\
(\mu \mathrm{g} / \mathrm{L}) \\
(01085) \\
\end{array}$ & $\begin{array}{c}\begin{array}{c}\text { Zinc } \\
(\mu \mathrm{g} / \mathrm{L}) \\
(01090)\end{array} \\
\end{array}$ \\
\hline Benchmark type & na & HBSL & HBSL & MCL-CA & MCL-US & HBSL & HBSL & MCL-US & NL-CA & HBSL \\
\hline Benchmark level & na & 300 & 40 & 100 & 50 & 100 & 4,000 & 30 & 50 & 2,000 \\
\hline [LT-MDL or SRL] & {$[0.22]$} & {$[0.66]^{1,2}$} & {$[0.05]^{1}$} & {$[0.21]^{1,2}$} & {$[0.05]^{1}$} & {$[0.02]^{1}$} & {$[0.8]^{1}$} & {$[0.014]^{1}$} & {$[0.08]$} & {$[6.2]^{1,2}$} \\
\hline \multicolumn{11}{|c|}{ Madera/Chowchilla-Kings Shallow Aquifer study unit (77 wells sampled)_Continued } \\
\hline \multicolumn{11}{|c|}{ Madera/Chowchilla study area (28 wells sampled)_-Continued } \\
\hline S3-MACK-M20 & 1.19 & - & 2.44 & 0.46 & 0.12 & - & 151 & 0.341 & 20.4 & 46.0 \\
\hline S3-MACK-M21 & 2.03 & 56.65 & 1.36 & 2.06 & 0.11 & - & 323 & 1.45 & 14.7 & 484 \\
\hline S3-MACK-M22 & 0.71 & 2.77 & 0.899 & 1.32 & 0.88 & - & 234 & 0.501 & 21.4 & 14.4 \\
\hline S3-MACK-M23 & 1.43 & - & 2.89 & 0.25 & - & - & 80.0 & 0.487 & 8.1 & 36.2 \\
\hline S3-MACK-M24 & 0.76 & - & 1.45 & 0.22 & 0.21 & - & 71.0 & 0.077 & 23.2 & 72.2 \\
\hline S3-MACK-M25 & 0.97 & 1.78 & 1.78 & - & 0.26 & - & 154 & 0.392 & 24.1 & 25.7 \\
\hline S3-MACK-M26 & 0.50 & - & 5.02 & 0.30 & 0.30 & - & 102 & 0.271 & 22.2 & 11.1 \\
\hline S3-MACK-M27 & 2.11 & 1.30 & 3.66 & 0.52 & 0.28 & - & 154 & 0.209 & 17.9 & 270 \\
\hline S3-MACK-M28 & 14.4 & 30.28 & 6.09 & 0.40 & - & - & 295 & - & 0.61 & 11.3 \\
\hline \multicolumn{11}{|c|}{ Kings study area (49 wells sampled)—Continued } \\
\hline S3-MACK-K01 & 4.28 & $* 516.6$ & $* 87.9$ & 1.97 & 0.21 & - & 807 & * 222 & 0.95 & 53.4 \\
\hline S3-MACK-K02 & 2.02 & 35.63 & 4.56 & 0.50 & 0.09 & - & 226 & 0.041 & 0.57 & 23.3 \\
\hline S3-MACK-K03 & 2.99 & 87.30 & 2.43 & 1.03 & 0.07 & - & 359 & 29.6 & 4.0 & 7.8 \\
\hline S3-MACK-K04 & 5.11 & * 496.6 & 1.43 & 0.52 & - & - & 567 & $* 285$ & 0.89 & 8.8 \\
\hline S3-MACK-K05 & 7.41 & 154.6 & 4.20 & 0.72 & 0.06 & - & 356 & * 147 & 1.0 & $\leq 2.4$ \\
\hline S3-MACK-K06 & 18.2 & $* 1,722$ & 3.52 & 2.71 & 0.07 & - & 969 & $* 154$ & 0.50 & 15.6 \\
\hline S3-MACK-K07 & 7.77 & $* 414.2$ & 7.62 & - & 0.21 & - & 315 & 10.1 & 3.0 & - \\
\hline S3-MACK-K08 & 57.6 & 1.62 & 7.01 & 2.31 & 1.8 & - & 1,050 & $* 250$ & 8.4 & 12.4 \\
\hline S3-MACK-K09 & 8.59 & 20.97 & 2.48 & - & 0.07 & - & 130 & - & 0.56 & - \\
\hline S3-MACK-K10 & 36.6 & 17.66 & 0.867 & 2.61 & 3.6 & - & 2,170 & $* 210$ & 12.7 & 71.5 \\
\hline
\end{tabular}


Table 7. Results for analyses of trace elements in groundwater samples collected for the Madera/Chowchilla-Kings Shallow Aquifer study unit, Groundwater Ambient Monitoring and Assessment (GAMA) Priority Basin Project, California, August 2013 to April 2014._Continued

[The five-digit USGS parameter code below the constituent name is used to uniquely identify a specific constituent or property. Samples from all 77 grid wells were analyzed. Constituents for which all samples were non-detections (thallium) or are reviewed and rejected (cobalt) are not listed. Information about the constituents given in table 3C. GAMA well identification numbers: S3-MACK-K, Madera/ Chowchilla-Kings Shallow Aquifer Kings study-area grid well; S3-MACK-M, Madera/Chowchilla-Kings Shallow Aquifer Madera/Chowchilla study area grid well. Benchmark type, benchmark level, and LT-MDL as of April 30, 2014. Benchmark types: Maximum contaminant level benchmarks are listed as MCL-US when the MCL-US and MCL-CA are identical, and as MCL-CA when the MCL-CA is lowe than the MCL-US or no MCL-US exists. AL-US, U.S. Environmental Protection Agency (EPA) action level; HBSL, USGS health-based screening level; MCL-CA, State of California maximum contaminant level; MCL-US, EPA maximum contaminant level; NL-CA, State of California notification level; SMCL-CA, State of California secondary maximum contaminant level. Abbreviations: LT-MDL, long-term method detection level; na, not available; SRL, study reporting level; USGS, U.S. Geological Survey; $\mu \mathrm{g} / \mathrm{L}$, micrograms per liter; —, not detected; $\leq$, less than or equal to; *, concentration greater than the benchmark level]

\begin{tabular}{|c|c|c|c|c|c|c|c|c|c|c|}
\hline $\begin{array}{c}\text { GAMA } \\
\text { identification } \\
\text { number }\end{array}$ & $\begin{array}{l}\text { Lithium } \\
\text { ( } \mu \mathrm{g} / \mathrm{L}) \\
(\mathbf{0 1 1 3 0})\end{array}$ & $\begin{array}{c}\text { Manganese } \\
(\mu \mathrm{g} / \mathrm{L}) \\
(01056)\end{array}$ & $\begin{array}{c}\text { Molybdenum } \\
(\mu \mathrm{g} / \mathrm{L}) \\
(01060)\end{array}$ & $\begin{array}{c}\text { Nickel } \\
\text { (pg/L) } \\
(01065)\end{array}$ & $\begin{array}{c}\text { Selenium } \\
(\mu \mathrm{g} / \mathrm{L}) \\
(01145)\end{array}$ & $\begin{array}{c}\text { Silver } \\
(\mu \mathrm{g} / \mathrm{L}) \\
(01075)\end{array}$ & $\begin{array}{c}\text { Strontium } \\
(\mu \mathrm{g} / \mathrm{L}) \\
(01080)\end{array}$ & $\begin{array}{c}\text { Uranium } \\
\text { ( } \mu \mathrm{g} / \mathrm{L}) \\
(22703)\end{array}$ & $\begin{array}{c}\text { Vanadium } \\
(\mu \mathrm{g} / \mathrm{L}) \\
(01085)\end{array}$ & $\begin{array}{c}\text { Zinc } \\
(\mu \mathrm{g} / \mathrm{L}) \\
(01090)\end{array}$ \\
\hline Benchmark type & na & HBSL & HBSL & MCL-CA & MCL-US & HBSL & HBSL & MCL-US & NL-CA & HBSL \\
\hline Benchmark level & na & 300 & 40 & 100 & 50 & 100 & 4,000 & 30 & 50 & 2,000 \\
\hline [LT-MDL or SRL] & {$[0.22]$} & {$[0.66]^{1,2}$} & {$[0.05]^{1}$} & {$[0.21]^{1,2}$} & {$[0.05]^{1}$} & {$[0.02]^{1}$} & {$[0.8]^{1}$} & {$[0.014]^{1}$} & {$[0.08]$} & {$[6.2]^{1,2}$} \\
\hline
\end{tabular}

Madera/Chowchilla-Kings Shallow Aquifer study unit (77 wells sampled)—Continued

Kings study area (49 wells sampled) - Continued

\begin{tabular}{|c|c|c|c|c|c|c|c|c|c|c|}
\hline S3-MACK-K11 & 34.5 & - & 0.761 & 0.44 & 0.88 & - & 706 & $* 80.4$ & 7.5 & 37.0 \\
\hline S3-MACK-K12 & 2.19 & 15.41 & 7.35 & 0.63 & 0.08 & - & 238 & 15.8 & 3.7 & $\leq 2.5$ \\
\hline S3-MACK-K13 & 1.35 & 15.07 & 16.5 & 0.68 & 0.06 & - & 98.0 & 0.228 & 0.48 & - \\
\hline S3-MACK-K14 & 0.60 & 3.01 & 7.28 & - & - & - & 26.1 & 0.084 & 0.20 & 22.4 \\
\hline S3-MACK-K15 & 1.97 & - & 0.485 & 0.47 & 1.4 & - & 170 & 28.7 & 4.8 & 12.0 \\
\hline S3-MACK-K16 & 5.37 & - & 0.209 & 3.30 & 0.62 & - & 1,710 & $* 142$ & 3.6 & 111 \\
\hline S3-MACK-K17 & 1.90 & - & 0.932 & 0.37 & - & - & 118 & 6.26 & 14.4 & $\leq 5.8$ \\
\hline S3-MACK-K18 & 25.0 & - & 1.32 & 0.78 & 0.14 & - & 902 & * 229 & 12.0 & 63.3 \\
\hline S3-MACK-K19 & 23.2 & - & 1.37 & 2.77 & 0.16 & - & 966 & * 169 & 18.5 & 44.6 \\
\hline S3-MACK-K20 & 16.3 & - & 2.34 & 1.08 & 0.96 & - & 670 & * 122 & 12.3 & 19.0 \\
\hline S3-MACK-K21 & 24.1 & - & 11.0 & 0.80 & 0.87 & - & 412 & * 61.4 & 16.2 & 18.7 \\
\hline S3-MACK-K22 & 8.03 & $\leq 0.45$ & 1.64 & 0.72 & 0.07 & - & 221 & 10.6 & 14.7 & 10.2 \\
\hline S3-MACK-K23 & 6.82 & - & 2.62 & 0.74 & - & - & 89.7 & 0.454 & 18.5 & - \\
\hline S3-MACK-K24 & 7.33 & - & 0.792 & 0.41 & 0.32 & - & 513 & $* 64.4$ & 27.6 & 6.6 \\
\hline S3-MACK-K25 & 2.76 & - & 1.59 & 0.25 & 0.16 & - & 198 & 14.2 & 10.4 & 24.2 \\
\hline S3-MACK-K26 & 1.30 & - & 1.01 & 0.39 & 0.06 & - & 143 & 9.26 & 10.2 & 8.5 \\
\hline S3-MACK-K27 & 4.86 & - & 0.634 & 1.09 & 0.90 & - & 955 & $* 74.4$ & 8.6 & 32.4 \\
\hline S3-MACK-K28 & 1.59 & - & 0.359 & 0.65 & 0.19 & - & 351 & 14.2 & 12.5 & - \\
\hline S3-MACK-K29 & 3.26 & $\leq 0.56$ & 1.32 & 0.84 & 0.41 & - & 647 & 11.7 & 37.2 & 27.5 \\
\hline
\end{tabular}


Table 7. Results for analyses of trace elements in groundwater samples collected for the Madera/Chowchilla-Kings Shallow Aquifer study unit, Groundwater Ambient Monitoring and Assessment (GAMA) Priority Basin Project, California, August 2013 to April 2014.-Continued

[The five-digit USGS parameter code below the constituent name is used to uniquely identify a specific constituent or property. Samples from all 77 grid wells were analyzed. Constituents for which all samples were non-detections (thallium) or are reviewed and rejected (cobalt) are not listed. Information about the constituents given in table 3C. GAMA well identification numbers: S3-MACK-K, Madera/ Chowchilla-Kings Shallow Aquifer Kings study-area grid well; S3-MACK-M, Madera/Chowchilla-Kings Shallow Aquifer Madera/Chowchilla study area grid well. Benchmark type, benchmark level, and LT-MDL as of April 30, 2014. Benchmark types: Maximum contaminant level benchmarks are listed as MCL-US when the MCL-US and MCL-CA are identical, and as MCL-CA when the MCL-CA is lower than the MCL-US or no MCL-US exists. AL-US, U.S. Environmental Protection Agency (EPA) action level; HBSL, USGS health-based screening level; MCL-CA, State of California maximum contaminant level; MCL-US, EPA maximum contaminant level; NL-CA, State of California notification level; SMCL-CA, State of California secondary maximum contaminant level. Abbreviations: LT-MDL, long-term method detection level; na, not available; SRL, study reporting level; USGS, U.S. Geological Survey; $\mu \mathrm{g} / \mathrm{L}$, micrograms per liter; - , not detected; $\leq$, less than or equal to; *, concentration greater than the benchmark level]

\begin{tabular}{|c|c|c|c|c|c|c|c|c|c|c|}
\hline $\begin{array}{c}\text { GAMA } \\
\text { identification } \\
\text { number }\end{array}$ & $\begin{array}{l}\text { Lithium } \\
\text { ( } \mu \mathrm{g} / \mathrm{L}) \\
(01130)\end{array}$ & $\begin{array}{c}\text { Manganese } \\
(\mu \mathrm{g} / \mathrm{L}) \\
(01056)\end{array}$ & $\begin{array}{c}\text { Molybdenum } \\
(\mu \mathrm{g} / \mathrm{L}) \\
(01060)\end{array}$ & $\begin{array}{c}\text { Nickel } \\
\text { ( } \mu \mathrm{g} / \mathrm{L}) \\
(01065)\end{array}$ & $\begin{array}{c}\text { Selenium } \\
(\mu \mathrm{g} / \mathrm{L}) \\
(01145)\end{array}$ & $\begin{array}{c}\text { Silver } \\
(\mu \mathrm{g} / \mathrm{L}) \\
(01075)\end{array}$ & $\begin{array}{c}\text { Strontium } \\
(\mu \mathrm{g} / \mathrm{L}) \\
(01080)\end{array}$ & $\begin{array}{c}\text { Uranium } \\
\text { ( } \mu \mathrm{g} / \mathrm{L}) \\
(22703)\end{array}$ & $\begin{array}{c}\text { Vanadium } \\
(\mu \mathrm{g} / \mathrm{L}) \\
(01085)\end{array}$ & $\begin{array}{c}\text { Zinc } \\
(\mu \mathrm{g} / \mathrm{L}) \\
(01090)\end{array}$ \\
\hline Benchmark type & na & HBSL & HBSL & MCL-CA & MCL-US & HBSL & HBSL & MCL-US & NL-CA & HBSL \\
\hline Benchmark level & na & 300 & 40 & 100 & 50 & 100 & 4,000 & 30 & 50 & 2,000 \\
\hline [LT-MDL or SRL] & {$[0.22]$} & {$[0.66]^{1,2}$} & {$[0.05]^{1}$} & {$[0.21]^{1,2}$} & {$[0.05]^{1}$} & {$[0.02]^{1}$} & {$[0.8]^{1}$} & {$[0.014]^{1}$} & {$[0.08]$} & {$[6.2]^{1,2}$} \\
\hline
\end{tabular}

Madera/Chowchilla-Kings Shallow Aquifer study unit (77 wells sampled)_Continued

\begin{tabular}{|c|c|c|c|c|c|c|c|c|c|c|}
\hline \multicolumn{11}{|c|}{ Kings study area (49 wells sampled)—Continued } \\
\hline S3-MACK-K30 & 2.06 & - & 3.60 & 0.37 & - & - & 124 & 0.855 & 40.3 & 7.5 \\
\hline S3-MACK-K31 & 0.75 & - & 3.12 & - & 0.24 & - & 103 & 0.306 & 41.9 & $\leq 2.7$ \\
\hline S3-MACK-K32 & 1.19 & - & 0.71 & - & 0.06 & - & 112 & 2.19 & 8.3 & $\leq 2.2$ \\
\hline S3-MACK-K33 & 2.48 & - & 0.573 & 0.76 & - & - & 538 & 10.6 & 18.8 & 13.8 \\
\hline S3-MACK-K34 & 5.34 & $\leq 0.44$ & 1.84 & 0.76 & 0.18 & - & 392 & 11.2 & 16.0 & $\leq 2.6$ \\
\hline S3-MACK-K35 & 0.91 & - & 2.32 & 0.7 & - & - & 110 & 0.641 & 20.9 & - \\
\hline S3-MACK-K36 & 1.50 & 0.80 & 2.33 & 3.71 & 0.09 & - & 220 & 2.67 & 16.3 & - \\
\hline S3-MACK-K37 & 1.39 & - & 0.585 & 0.49 & - & - & 99.8 & 0.388 & 30.2 & - \\
\hline S3-MACK-K38 & 5.30 & - & 0.144 & 0.62 & 0.06 & - & 540 & 3.05 & 11.4 & $\leq 2.1$ \\
\hline S3-MACK-K39 & 1.83 & - & 0.896 & 0.55 & 0.20 & - & 588 & 20.5 & 7.7 & $\leq 4.6$ \\
\hline S3-MACK-K40 & 6.87 & - & 0.597 & 0.80 & 0.08 & - & 705 & $* 42.1$ & 16.7 & 11.7 \\
\hline S3-MACK-K41 & - & - & 2.56 & 0.83 & 0.11 & - & 505 & 5.63 & 26.6 & 44.4 \\
\hline S3-MACK-K42 & 1.78 & - & 1.78 & 0.44 & 0.56 & - & 933 & 10.4 & $* 65.0$ & 15.6 \\
\hline S3-MACK-K43 & 1.00 & $\leq 0.51$ & 2.00 & 1.07 & - & - & 486 & 7.57 & 38.2 & 211 \\
\hline S3-MACK-K44 & 5.13 & - & 1.68 & 1.16 & 0.09 & 0.029 & 837 & 16.3 & 20.0 & $\leq 3.5$ \\
\hline S3-MACK-K45 & 1.55 & $\leq 0.51$ & 3.79 & 0.36 & 0.09 & - & 375 & 4.93 & $* 59.9$ & 7.2 \\
\hline S3-MACK-K46 & 0.48 & 9.46 & 0.073 & 1.04 & - & - & 406 & 7.85 & 1.4 & 13.7 \\
\hline S3-MACK-K47 & 7.22 & $\leq 0.43$ & 0.303 & 1.26 & 0.20 & 0.123 & 698 & 4.86 & 17.2 & 29.9 \\
\hline S3-MACK-K48 & 5.62 & - & 1.43 & 0.46 & 0.32 & - & 275 & 3.59 & 41.2 & 11.7 \\
\hline
\end{tabular}


Table 7. Results for analyses of trace elements in groundwater samples collected for the Madera/Chowchilla-Kings Shallow Aquifer study unit, Groundwater Ambient Monitoring and Assessment (GAMA) Priority Basin Project, California, August 2013 to April 2014._Continued

[The five-digit USGS parameter code below the constituent name is used to uniquely identify a specific constituent or property. Samples from all 77 grid wells were analyzed. Constituents for which all samples were non-detections (thallium) or are reviewed and rejected (cobalt) are not listed. Information about the constituents given in table 3C. GAMA well identification numbers: S3-MACK-K, Madera/ Chowchilla-Kings Shallow Aquifer Kings study-area grid well; S3-MACK-M, Madera/Chowchilla-Kings Shallow Aquifer Madera/Chowchilla study area grid well. Benchmark type, benchmark level, and LT-MDL as of April 30, 2014. Benchmark types: Maximum contaminant level benchmarks are listed as MCL-US when the MCL-US and MCL-CA are identical, and as MCL-CA when the MCL-CA is lower than the MCL-US or no MCL-US exists. AL-US, U.S. Environmental Protection Agency (EPA) action level; HBSL, USGS health-based screening level; MCL-CA, State of California maximum contaminant level; MCL-US, EPA maximum contaminant level; NL-CA, State of California notification level; SMCL-CA, State of California secondary maximum contaminant level. Abbreviations: LT-MDL, long-term method detection level; na, not available; SRL, study reporting level; USGS, U.S. Geological Survey; $\mu \mathrm{g} / \mathrm{L}$, micrograms per liter; 一, not detected; $\leq$, less than or equal to; *, concentration greater than the benchmark level]

\begin{tabular}{|c|c|c|c|c|c|c|c|c|c|c|}
\hline $\begin{array}{c}\text { GAMA } \\
\text { identification } \\
\text { number }\end{array}$ & $\begin{array}{c}\text { Lithium } \\
(\mu \mathrm{g} / \mathrm{L}) \\
(01130)\end{array}$ & $\begin{array}{c}\text { Manganese } \\
(\mu \mathrm{g} / \mathrm{L}) \\
(01056)\end{array}$ & $\begin{array}{c}\text { Molybdenum } \\
(\mu \mathrm{g} / \mathrm{L}) \\
(01060)\end{array}$ & $\begin{array}{c}\text { Nickel } \\
\text { ( } \mu \mathrm{g} / \mathrm{L}) \\
(01065)\end{array}$ & $\begin{array}{c}\text { Selenium } \\
(\mu \mathrm{g} / \mathrm{L}) \\
(01145)\end{array}$ & $\begin{array}{c}\text { Silver } \\
\text { ( } \mu \mathrm{g} / \mathrm{L}) \\
(01075)\end{array}$ & $\begin{array}{c}\text { Strontium } \\
(\mu \mathrm{g} / \mathrm{L}) \\
(01080) \\
\end{array}$ & $\begin{array}{c}\text { Uranium } \\
\text { ( } \mu \mathrm{g} / \mathrm{L}) \\
(22703)\end{array}$ & $\begin{array}{c}\text { Vanadium } \\
(\mu \mathrm{g} / \mathrm{L}) \\
(01085) \\
\end{array}$ & $\begin{array}{c}\text { Zinc } \\
(\mu \mathrm{g} / \mathrm{L}) \\
(01090)\end{array}$ \\
\hline Benchmark type & na & HBSL & HBSL & MCL-CA & MCL-US & HBSL & HBSL & MCL-US & NL-CA & HBSL \\
\hline Benchmark level & na & 300 & 40 & 100 & 50 & 100 & 4,000 & 30 & 50 & 2,000 \\
\hline [LT-MDL or SRL] & {$[0.22]$} & {$[0.66]^{1,2}$} & {$[0.05]^{1}$} & {$[0.21]^{1,2}$} & {$[0.05]^{1}$} & {$[0.02]^{1}$} & {$[0.8]^{1}$} & {$[0.014]^{1}$} & {$[0.08]$} & {$[6.2]^{1,2}$} \\
\hline \multicolumn{11}{|c|}{ Madera/Chowchilla-Kings Shallow Aquifer study unit (77 wells sampled)—Continued } \\
\hline \multicolumn{11}{|c|}{ Kings study area ( 49 wells sampled)_Continued } \\
\hline S3-MACK-K49 & 4.19 & - & 0.859 & 1.03 & 0.42 & - & 430 & 7.24 & 32.2 & 62.4 \\
\hline
\end{tabular}

${ }^{1}$ The LT-MDL changed during the period that samples were collected for this study (see table $3 C$ ). The higher of the two LT-MDLs is reported here, and detections at concentrations less than this LT-MDL were replaced with a - symbol, indicating a non-detection relative to the higher LT-MDL.

${ }^{2}$ The reporting level is the SRL defined by Davis and others (2014). Results at concentrations less than an SRL were reclassified as less or equal to the concentration reported by the laboratory and marked with a symbol.

${ }^{3}$ The laboratory raised reporting levels for these samples because of problems due to matrix interferences (aluminum: $2.2 \mu \mathrm{g} / \mathrm{L}$ to $4.4 \mu \mathrm{g} / \mathrm{L}$ for S3-MACK-K01, -K10, and 2.2 $\mu \mathrm{g} / \mathrm{L}$ to $11 \mu \mathrm{g} / \mathrm{L}$ for S3-MACKK33; antimony: $0.027 \mu \mathrm{g} / \mathrm{L}$ to $0.135 \mu \mathrm{g} / \mathrm{L}$ for S3-MACK-K33; beryllium: $0.02 \mu \mathrm{g} / \mathrm{L}$ to $0.04 \mu \mathrm{g} / \mathrm{L}$ for S3-MACK-K01; chromium: $0.3 \mu \mathrm{g} / \mathrm{L}$ to $0.6 \mu \mathrm{g} / \mathrm{L}$ for S3-MACK-K01; copper: $0.8 \mu \mathrm{g} / \mathrm{L}$ to $4.0 \mu \mathrm{g} / \mathrm{L}$ [note SRL is $2.1 \mu \mathrm{g} / \mathrm{L}$ ] for S3-MACK-K33; manganese: $0.40 \mu \mathrm{g} / \mathrm{L}$ to $0.75 \mu \mathrm{g} / \mathrm{L}$ [note SRL is $0.66 \mu \mathrm{g} / \mathrm{L}$ ] for S3-MACK-K33; nickel: $0.20 \mu \mathrm{g} / \mathrm{L}$ to $0.8 \mu \mathrm{g} / \mathrm{L}$ [note SRL is $0.21 \mu \mathrm{g} / \mathrm{L}$ ] for S3-MACK-K07; silver: 0.02 $\mu \mathrm{g} / \mathrm{L}$ to $0.04 \mu \mathrm{g} / \mathrm{L}$ for S3-MACK-K01; thallium: $0.03 \mu \mathrm{g} / \mathrm{L}$ to $0.06 \mu \mathrm{g} / \mathrm{L}$ for S3-MACK-K01) 
Table 8. Results for analyses of nutrients in groundwater samples collected for the Madera/Chowchilla-Kings Shallow Aquifer study unit, Groundwater Ambient Monitoring and Assessment (GAMA) Priority Basin Project, California, August 2013 to April 2014.

[The five-digit USGS parameter code below the constituent name is used to uniquely identify a specific constituent or property. Samples from all 77 grid wells were analyzed. Information about the constituents given in table 3C. GAMA well identification numbers: S3-MACK-K, Madera/Chowchilla-Kings Shallow Aquifer Kings study-area grid well; S3-MACK-M, Madera/Chowchilla-Kings Shallow Aquifer Madera/Chowchilla study-area grid well. Benchmark type, benchmark level, and LT-MDL as of April 30, 2014. Benchmark types: HAL-US, U.S. Environmental Protection Agency (EPA) lifetime health advisory level; MCL-US, EPA maximum contaminant level. Abbreviations: LT-MDL, long-term method detection level; mg/L, milligrams per liter; na, not available; USGS, U.S. Geological Survey; *, concentration greater than the benchmark level; —, not detected]

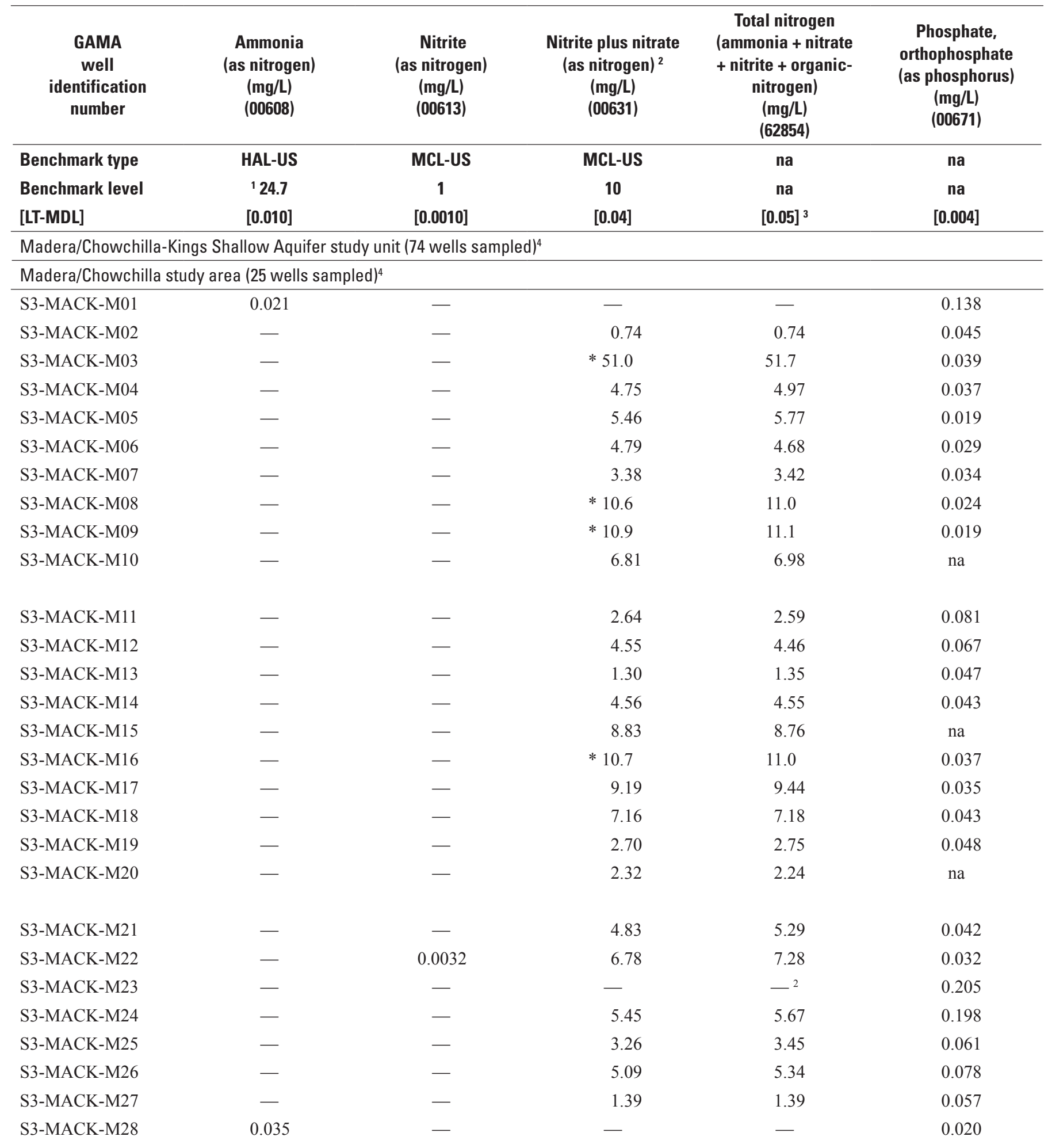


Table 8. Results for analyses of nutrients in groundwater samples collected for the Madera/Chowchilla-Kings Shallow Aquifer study unit, Groundwater Ambient Monitoring and Assessment (GAMA) Priority Basin Project, California, August 2013 to April 2014.—Continued

[The five-digit USGS parameter code below the constituent name is used to uniquely identify a specific constituent or property. Samples from all 77 grid wells were analyzed. Information about the constituents given in table 3C. GAMA well identification numbers: S3-MACK-K, Madera/Chowchilla-Kings Shallow Aquifer Kings study-area grid well; S3-MACK-M, Madera/Chowchilla-Kings Shallow Aquifer Madera/Chowchilla study-area grid well. Benchmark type, benchmark level, and LT-MDL as of April 30, 2014. Benchmark types: HAL-US, U.S. Environmental Protection Agency (EPA) lifetime health advisory level; MCL-US, EPA maximum contaminant level. Abbreviations: LT-MDL, long-term method detection level; mg/L, milligrams per liter; na, not available; USGS, U.S. Geological Survey; *, concentration greater than the benchmark level; - , not detected]

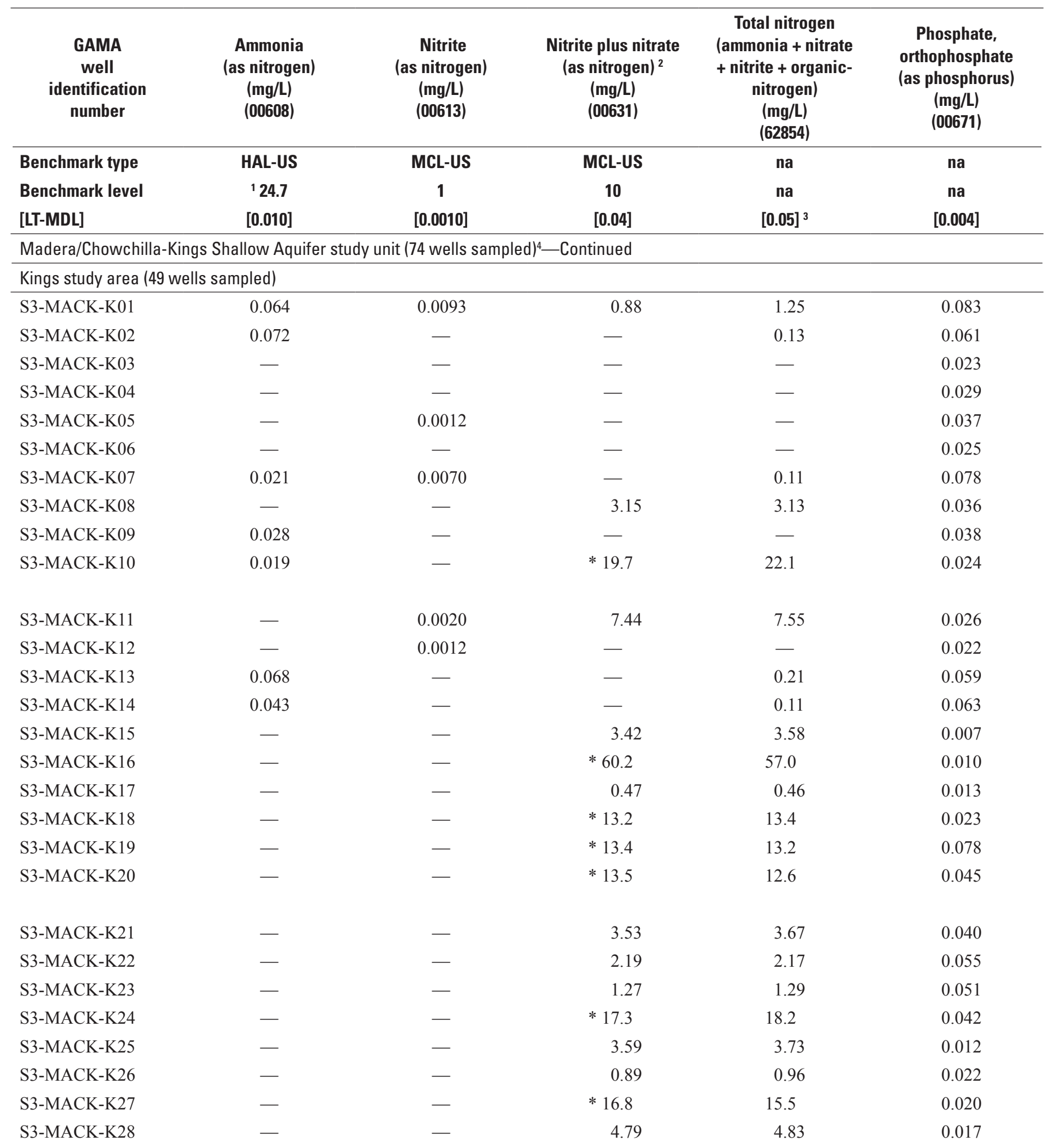


Table 8. Results for analyses of nutrients in groundwater samples collected for the Madera/Chowchilla-Kings Shallow Aquifer study unit, Groundwater Ambient Monitoring and Assessment (GAMA) Priority Basin Project, California, August 2013 to April 2014.-Continued

[The five-digit USGS parameter code below the constituent name is used to uniquely identify a specific constituent or property. Samples from all 77 grid wells were analyzed. Information about the constituents given in table 3C. GAMA well identification numbers: S3-MACK-K, Madera/Chowchilla-Kings Shallow Aquifer Kings study-area grid well; S3-MACK-M, Madera/Chowchilla-Kings Shallow Aquifer Madera/Chowchilla study-area grid well. Benchmark type, benchmark level, and LT-MDL as of April 30, 2014. Benchmark types: HAL-US, U.S. Environmental Protection Agency (EPA) lifetime health advisory level; MCL-US, EPA maximum contaminant level. Abbreviations: LT-MDL, long-term method detection level; mg/L, milligrams per liter; na, not available; USGS, U.S. Geological Survey; *, concentration greater than the benchmark level; —, not detected]

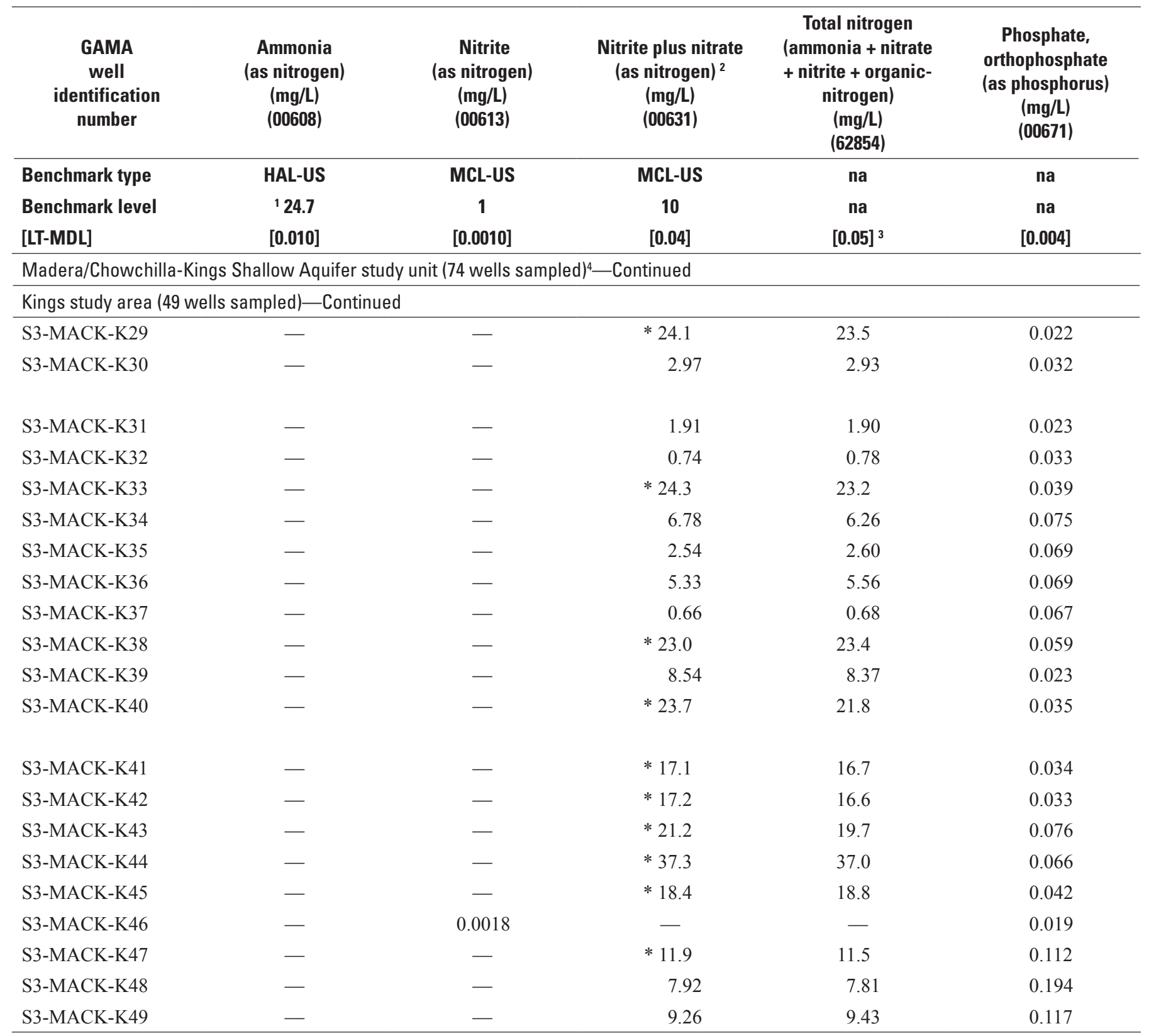

${ }^{1}$ The HAL-US is $30 \mathrm{mg} / \mathrm{L}$ "as ammonia." To facilitate comparison to the analytical results, we converted and reported this HAL-US as $24.7 \mathrm{mg} / \mathrm{L}$ "as nitrogen."

${ }^{2}$ Nitrite plus nitrate (as nitrogen) is referred to as nitrate in the text.

${ }^{3}$ The laboratory raised the reporting level for one sample because of interference (total nitrogen: $0.05 \mathrm{mg} / \mathrm{L}$ to $0.1 \mathrm{mg} / \mathrm{L}$ for S3-MACK-M23).

${ }^{4}$ Results for phosphate are not available for three samples in the Madera/Chowchilla study area: S3-MACK-10, S3-MACK-15, and S3-MACK-20 (see appendix). 
Table 9. Results for analyses of major and minor ions, silica, and total dissolved solids (TDS) in groundwater samples collected for the Madera/Chowchilla-Kings Shallow Aquifer study unit, Groundwater Ambient Monitoring and Assessment (GAMA) Priority Basin Project, California, August 2013 to April 2014.

[The five-digit USGS parameter code below the constituent name is used to uniquely identify a specific constituent or property. Samples from all 77 grid wells were analyzed, unless noted as not collected (nc). Information about the constituents given in table 3C. GAMA well identification numbers: S3-MACK-K, Madera/Chowchilla-Kings Shallow Aquifer Kings study-area grid well; S3-MACK-M, Madera/Chowchilla-Kings Shallow Aquifer Madera/Chowchilla study-area grid well. Benchmark type, benchmark level, and LT-MDL as of April 30, 2014. Benchmark types: Maximum contaminant level benchmarks are listed as MCL-US when the MCL-US and MCL-CA are identical, and as MCL-CA when the MCL-CA is lower than the MCL-US or no MCL-US exists. The SMCL-CA benchmarks for chloride, sulfate, and TDS are the upper benchmark levels. MCL-CA, State of California maximum contaminant level; SMCL-CA, State of California secondary maximum contaminant level. Abbreviations: E, estimated or having a higher degree of uncertainty; LT-MDL, long-term method detection level; mg/L, milligrams per liter; na, not available; nc, not collected; $\mathrm{SiO}_{2}$, silicon dioxide; USGS, U.S. Geological Survey; —, not detected; * concentration greater than benchmark level]

\begin{tabular}{|c|c|c|c|c|c|c|c|c|c|c|c|}
\hline $\begin{array}{c}\text { GAMA } \\
\text { well } \\
\text { identification } \\
\text { number }\end{array}$ & $\begin{array}{c}\text { Calcium } \\
\text { (mg/L) } \\
(00915)\end{array}$ & $\begin{array}{c}\text { Magnesium } \\
(\mathrm{mg} / \mathrm{L}) \\
(00925)\end{array}$ & $\begin{array}{c}\text { Potassium } \\
\text { (mg/L) } \\
(00935)\end{array}$ & $\begin{array}{c}\text { Sodium } \\
\text { (mg/L) } \\
(00930)\end{array}$ & $\begin{array}{c}\text { Bromide } \\
\text { (mg/L) } \\
(71870)\end{array}$ & $\begin{array}{c}\text { Fluoride } \\
\text { (mg/L) } \\
(00950)\end{array}$ & $\begin{array}{l}\text { lodide } \\
\text { (mg/L) } \\
(71865)\end{array}$ & $\begin{array}{c}\text { Chloride } \\
\text { (mg/L) } \\
(00940)\end{array}$ & $\begin{array}{l}\text { Sulfate } \\
\text { (mg/L) } \\
(00945)\end{array}$ & $\begin{array}{c}\text { Silica } \\
\left.\text { (as } \mathrm{SiO}_{2}\right) \\
(\mathrm{mg} / \mathrm{L}) \\
(00955)\end{array}$ & $\begin{array}{c}\text { TDS } \\
(\mathrm{mg} / \mathrm{L}) \\
(70300)\end{array}$ \\
\hline Benchmark type & na & na & na & na & na & MCL-CA & na & SMCL-CA & SMCL-CA & na & SMCL-CA \\
\hline Benchmark level & na & na & na & na & na & 2 & na & 500 & 500 & na & 1,000 \\
\hline [LT-MDL] & {$[0.022]$} & {$[0.011]$} & {$[0.03]$} & {$[0.06]$} & {$[0.03]^{1}$} & {$[0.01]$} & {$[0.001]$} & {$[0.06]^{1}$} & {$[0.09]^{1}$} & {$[0.018]$} & [10] \\
\hline \multicolumn{12}{|c|}{ Madera/Chowchilla-Kings Shallow Aquifer study unit (77 wells sampled) } \\
\hline \multicolumn{12}{|c|}{ Madera/Chowchilla study area (28 wells sampled) } \\
\hline S3-MACK-M01 & 14.1 & 1.99 & 1.77 & 6.62 & - & 0.15 & 0.024 & 2.17 & 5.78 & 52.7 & 109 \\
\hline S3-MACK-M02 & 23.8 & 6.98 & 2.71 & 25.3 & 0.051 & 0.15 & 0.004 & 36.7 & 6.91 & 70.9 & 238 \\
\hline S3-MACK-M03 & 200 & 48.2 & 3.96 & 193 & 0.552 & 0.07 & 0.033 & 250 & 52.9 & 63.4 & $* 1,250$ \\
\hline S3-MACK-M04 & 88.5 & 24.2 & 4.13 & 46.8 & 0.153 & 0.15 & 0.007 & 68.9 & 40.0 & 68.2 & 517 \\
\hline S3-MACK-M05 & 117 & 19.1 & 5.42 & 55.5 & 0.170 & 0.06 & 0.004 & 115 & 26.9 & 66.2 & 548 \\
\hline S3-MACK-M06 & 66.4 & 12.8 & 4.44 & 61.4 & 0.118 & 0.07 & 0.003 & 68.1 & 18.1 & 63.1 & 443 \\
\hline S3-MACK-M07 & 49.0 & 9.27 & 3.17 & 14.1 & 0.042 & 0.09 & 0.002 & 22.3 & 6.64 & 67.7 & 271 \\
\hline S3-MACK-M08 & 83.7 & 20.9 & 4.26 & 40.8 & 0.143 & 0.07 & 0.003 & 78.8 & 21.9 & 61.0 & 440 \\
\hline S3-MACK-M09 & 60.7 & 19.4 & 5.50 & 33.5 & 0.136 & 0.13 & 0.003 & 26.5 & 17.0 & 71.2 & 415 \\
\hline S3-MACK-M10 & 99.0 & 32.7 & 6.05 & 54.9 & 0.157 & 0.09 & 0.005 & 95.1 & 22.6 & 65.3 & 571 \\
\hline S3-MACK-M11 & 47.9 & 16.0 & 2.13 & 27.2 & 0.090 & 0.13 & 0.002 & 33.4 & 15.4 & 70.4 & 328 \\
\hline S3-MACK-M12 & 29.5 & 10.1 & 2.82 & 37.7 & 0.096 & 0.12 & 0.001 & 22.9 & 17.2 & 74.2 & 300 \\
\hline S3-MACK-M13 & 21.5 & 7.44 & 3.79 & 21.6 & 0.041 & 0.13 & 0.002 & 14.8 & 15.2 & 68.7 & 212 \\
\hline S3-MACK-M14 & 24.3 & 8.07 & 3.40 & 24.1 & 0.058 & 0.14 & 0.001 & 13.3 & 8.33 & 65.5 & 223 \\
\hline S3-MACK-M15 & 29.0 & 10.8 & 3.75 & 24.4 & 0.051 & 0.16 & 0.002 & 10.0 & 7.81 & 73.6 & 269 \\
\hline S3-MACK-M16 & 95.9 & 26.5 & 4.79 & 87.0 & 0.098 & 0.05 & 0.007 & 44.2 & 18.8 & 64.3 & 507 \\
\hline S3-MACK-M17 & 131 & 38.6 & 4.04 & 64.0 & 0.240 & 0.07 & 0.005 & 128 & 21.3 & 63.0 & 670 \\
\hline S3-MACK-M18 & 34.7 & 10.3 & 2.15 & 24.1 & 0.086 & 0.12 & 0.001 & 30.1 & 5.92 & 56.9 & 263 \\
\hline S3-MACK-M19 & 23.6 & 8.79 & 3.33 & 24.7 & 0.066 & 0.20 & 0.001 & 17.8 & 6.00 & 79.6 & 238 \\
\hline
\end{tabular}


Table 9. Results for analyses of major and minor ions, silica, and total dissolved solids (TDS) in groundwater samples collected for the Madera/Chowchilla-Kings Shallow Aquifer study unit, Groundwater Ambient Monitoring and Assessment (GAMA) Priority Basin Project, California, August 2013 to April 2014.—Continued

[The five-digit USGS parameter code below the constituent name is used to uniquely identify a specific constituent or property. Samples from all 77 grid wells were analyzed, unless noted as not collected (nc). Information about the constituents given in table 3C. GAMA well identification numbers: S3-MACK-K, Madera/Chowchilla-Kings Shallow Aquifer Kings study-area grid well; S3-MACK-M, Madera/Chowchilla-Kings Shallow Aquifer Madera/Chowchilla study-area grid well. Benchmark type, benchmark level, and LT-MDL as of April 30, 2014. Benchmark types: Maximum contaminant level benchmarks are listed as MCL-US when the MCL-US and MCL-CA are identical, and as MCL-CA when the MCL-CA is lower than the MCL-US or no MCL-US exists. The SMCL-CA benchmarks for chloride, sulfate, and TDS are the upper benchmark levels. MCL-CA, State of California maximum contaminant level; SMCL-CA, State of California secondary maximum contaminant level.

Abbreviations: E, estimated or having a higher degree of uncertainty; LT-MDL, long-term method detection level; $\mathrm{mg} / \mathrm{L}$, milligrams per liter; na, not available; nc, not collected; SiO ${ }_{2}$, silicon dioxide; USGS, U.S. Geological Survey; - , not detected; * concentration greater than benchmark level]

\begin{tabular}{|c|c|c|c|c|c|c|c|c|c|c|c|}
\hline $\begin{array}{c}\text { GAMA } \\
\text { well } \\
\text { identification } \\
\text { number }\end{array}$ & $\begin{array}{c}\text { Calcium } \\
\text { (mg/L) } \\
(00915)\end{array}$ & $\begin{array}{c}\text { Magnesium } \\
(\mathrm{mg} / \mathrm{L}) \\
(00925)\end{array}$ & $\begin{array}{l}\text { Potassium } \\
\text { (mg/L) } \\
(00935)\end{array}$ & $\begin{array}{c}\text { Sodium } \\
(\mathrm{mg} / \mathrm{L}) \\
(00930)\end{array}$ & $\begin{array}{c}\text { Bromide } \\
\text { (mg/L) } \\
(71870)\end{array}$ & $\begin{array}{c}\text { Fluoride } \\
\text { (mg/L) } \\
(00950)\end{array}$ & $\begin{array}{l}\text { lodide } \\
\text { (mg/L) } \\
(71865)\end{array}$ & $\begin{array}{c}\text { Chloride } \\
\text { (mg/L) } \\
(00940)\end{array}$ & $\begin{array}{c}\text { Sulfate } \\
\text { (mg/L) } \\
(00945)\end{array}$ & $\begin{array}{c}\text { Silica } \\
\left.\text { (as SiO })_{2}\right) \\
(\mathrm{mg} / \mathrm{L}) \\
(00955)\end{array}$ & $\begin{array}{c}\text { TDS } \\
(\mathrm{mg} / \mathrm{L}) \\
(70300)\end{array}$ \\
\hline Benchmark type & na & na & na & na & na & MCL-CA & na & SMCL-CA & SMCL-CA & na & SMCL-CA \\
\hline Benchmark level & na & na & na & na & na & 2 & na & 500 & 500 & na & 1,000 \\
\hline [LT-MDL] & [0.022] & [0.011] & {$[0.03]$} & {$[0.06]$} & {$[0.03]^{1}$} & [0.01] & {$[0.001]$} & {$[0.06]^{1}$} & {$[0.09]^{1}$} & [0.018] & [10] \\
\hline \multicolumn{12}{|c|}{ Madera/Chowchilla-Kings Shallow Aquifer study unit (77 wells sampled)_Continued } \\
\hline \multicolumn{12}{|c|}{ Madera/Chowchilla study area (28 wells) _-Continued } \\
\hline S3-MACK-M20 & 17.6 & 5.36 & 2.58 & 20.0 & 0.047 & 0.15 & 0.001 & 17.9 & 3.74 & 57.7 & 177 \\
\hline S3-MACK-M21 & 38.1 & 13.3 & 5.64 & 28.3 & 0.085 & 0.14 & 0.006 & 38.6 & 32.1 & 66.5 & 306 \\
\hline S3-MACK-M22 & 27.4 & 11.4 & 4.09 & 25.3 & 0.142 & 0.12 & 0.002 & 38.5 & 12.1 & 69.4 & 267 \\
\hline S3-MACK-M23 & 9.16 & 3.28 & 1.81 & 7.54 & - & 0.21 & 0.008 & 2.93 & 1.17 & 34.8 & 80 \\
\hline S3-MACK-M24 & 8.28 & 3.12 & 2.08 & 16.9 & 0.039 & 0.31 & - & 5.59 & 3.07 & 88.5 & 180 \\
\hline S3-MACK-M25 & 18.0 & 6.07 & 2.82 & 25.7 & 0.079 & 0.26 & - & 18.3 & 3.56 & 70.8 & 206 \\
\hline S3-MACK-M26 & 13.4 & 3.98 & 2.49 & 40.0 & 0.115 & 0.43 & 0.005 & 31.8 & 7.41 & 75.4 & 231 \\
\hline S3-MACK-M27 & 18.4 & 5.93 & 1.67 & 23.2 & 0.056 & 0.22 & 0.001 & 15.1 & 4.79 & 63.2 & 176 \\
\hline S3-MACK-M28 & 38.4 & 8.15 & 3.02 & 32.0 & 0.093 & 0.23 & 0.042 & 15.0 & 47.1 & 66.5 & 293 \\
\hline \multicolumn{12}{|c|}{ Kings study area (49 wells sampled) } \\
\hline S3-MACK-K01 & 95.6 & 33.1 & 3.78 & 385 & 0.180 & 0.36 & 0.064 & 99.7 & $* 652$ & 19.7 & $* 1,550$ \\
\hline S3-MACK-K02 & 27.3 & 1.24 & 0.55 & 349 & 0.657 & ${ }^{2} 1.05$ & 0.086 & 193 & 374 & 16.7 & $* 1,070$ \\
\hline S3-MACK-K03 & 57.7 & 3.38 & 1.97 & 50.2 & 0.154 & 0.10 & 0.052 & 68.9 & 45.6 & 41.9 & 347 \\
\hline S3-MACK-K04 & 80.7 & 7.90 & 1.26 & 115 & 0.143 & 0.10 & $\mathrm{nc}$ & 42.5 & 128 & 35.0 & 498 \\
\hline S3-MACK-K05 & 42.5 & 5.24 & 1.24 & 155 & 0.167 & 0.22 & 0.065 & 51.1 & 100 & 36.5 & 591 \\
\hline S3-MACK-K06 & 172 & 26.5 & 1.95 & 314 & 0.377 & 0.18 & 0.103 & 168 & * 703 & 36.4 & $* 1,580$ \\
\hline S3-MACK-K07 & 28.4 & 4.70 & 2.68 & 290 & 1.11 & 0.40 & 0.392 & 280 & 107 & 28.6 & 897 \\
\hline S3-MACK-K08 & 125 & 14.3 & 10.9 & 122 & 0.270 & 0.03 & 0.012 & 81.0 & 147 & 62.1 & 780 \\
\hline S3-MACK-K09 & 8.86 & 0.446 & 4.92 & 203 & 0.634 & 0.13 & 0.065 & 205 & 38.8 & 52.5 & 610 \\
\hline S3-MACK-K10 & 297 & 88.7 & 18.9 & 62.8 & 0.624 & - $^{(3)}$ & $\mathrm{nc}$ & 465 & 199 & 63.4 & $* 1,420$ \\
\hline
\end{tabular}


Table 9. Results for analyses of major and minor ions, silica, and total dissolved solids (TDS) in groundwater samples collected for the Madera/Chowchilla-Kings Shallow Aquifer study unit, Groundwater Ambient Monitoring and Assessment (GAMA) Priority Basin Project, California, August 2013 to April 2014.—Continued

[The five-digit USGS parameter code below the constituent name is used to uniquely identify a specific constituent or property. Samples from all 77 grid wells were analyzed, unless noted as not collected (nc). Information about the constituents given in table 3C. GAMA well identification numbers: S3-MACK-K, Madera/Chowchilla-Kings Shallow Aquifer Kings study-area grid well; S3-MACK-M, Madera/Chowchilla-Kings Shallow Aquifer Madera/Chowchilla study-area grid well. Benchmark type, benchmark level, and LT-MDL as of April 30, 2014. Benchmark types: Maximum contaminant level benchmarks are listed as MCL-US when the MCL-US and MCL-CA are identical, and as MCL-CA when the MCL-CA is lower than the MCL-US or no MCL-US exists. The SMCL-CA benchmarks for chloride, sulfate, and TDS are the upper benchmark levels. MCL-CA, State of California maximum contaminant level; SMCL-CA, State of California secondary maximum contaminant level.

Abbreviations: E, estimated or having a higher degree of uncertainty; LT-MDL, long-term method detection level; mg/L, milligrams per liter; na, not available; nc, not collected; SiO, silicon dioxide; USGS, U.S. Geological Survey; - , not detected; * concentration greater than benchmark level]

\begin{tabular}{|c|c|c|c|c|c|c|c|c|c|c|c|}
\hline $\begin{array}{c}\text { GAMA } \\
\text { well } \\
\text { identification } \\
\text { number }\end{array}$ & $\begin{array}{c}\text { Calcium } \\
\text { (mg/L) } \\
(00915)\end{array}$ & $\begin{array}{c}\text { Magnesium } \\
\text { (mg/L) } \\
(00925)\end{array}$ & $\begin{array}{c}\text { Potassium } \\
\text { (mg/L) } \\
(00935)\end{array}$ & $\begin{array}{c}\text { Sodium } \\
\text { (mg/L) } \\
(00930)\end{array}$ & $\begin{array}{c}\text { Bromide } \\
\text { (mg/L) } \\
(71870)\end{array}$ & $\begin{array}{c}\text { Fluoride } \\
\text { (mg/L) } \\
(00950)\end{array}$ & $\begin{array}{l}\text { lodide } \\
\text { (mg/L) } \\
(71865)\end{array}$ & $\begin{array}{c}\text { Chloride } \\
\text { (mg/L) } \\
(00940)\end{array}$ & $\begin{array}{c}\text { Sulfate } \\
\text { (mg/L) } \\
(00945)\end{array}$ & $\begin{array}{c}\text { Silica } \\
\text { (as SiO}{ }_{2} \text { ) } \\
(\mathrm{mg} / \mathrm{L}) \\
(00955)\end{array}$ & $\begin{array}{c}\text { TDS } \\
(\mathrm{mg} / \mathrm{L}) \\
(70300)\end{array}$ \\
\hline Benchmark type & na & na & na & na & na & MCL-CA & na & SMCL-CA & SMCL-CA & na & SMCL-CA \\
\hline Benchmark level & na & na & na & na & na & 2 & na & 500 & 500 & na & 1,000 \\
\hline [LT-MDL] & {$[0.022]$} & {$[0.011]$} & {$[0.03]$} & {$[0.06]$} & {$[0.03]^{1}$} & {$[0.01]$} & {$[0.001]$} & {$[0.06]^{1}$} & {$[0.09]^{1}$} & {$[0.018]$} & [10] \\
\hline \multicolumn{12}{|c|}{ Madera/Chowchilla-Kings Shallow Aquifer study unit (77 wells sampled)—Continued } \\
\hline \multicolumn{12}{|c|}{ Kings study area (49 wells sampled)_Continued } \\
\hline S3-MACK-K11 & 84.1 & 6.19 & 13.7 & 86.9 & 0.283 & 0.08 & $\mathrm{nc}$ & 139 & 58.9 & 63.1 & 610 \\
\hline S3-MACK-K12 & 38.0 & 0.414 & 0.83 & 83.0 & 0.394 & 0.21 & 0.094 & 120 & 21.4 & 23.3 & 343 \\
\hline S3-MACK-K13 & 11.2 & 0.426 & 0.44 & 247 & 0.317 & ${ }^{2} 1.07$ & 0.055 & 102 & 108 & 20.8 & 689 \\
\hline S3-MACK-K14 & 3.81 & 0.156 & 0.33 & 129 & 0.046 & 0.28 & 0.031 & 27.2 & 54.5 & 18.5 & 357 \\
\hline S3-MACK-K15 & 24.8 & 0.304 & 0.39 & 31.9 & - & 0.04 & - & 3.50 & 11.4 & 18.8 & 169 \\
\hline S3-MACK-K16 & 179 & 14.2 & 4.15 & 97.5 & 0.544 & 0.02 & 0.014 & 64.7 & 108 & 29.6 & 947 \\
\hline S3-MACK-K17 & 21.8 & 1.19 & 0.82 & 6.49 & - & 0.13 & - & 0.82 & 1.96 & 24.2 & 95 \\
\hline S3-MACK-K18 & 121 & 33.4 & 16.3 & 92.4 & 0.225 & 0.03 & $\mathrm{nc}$ & 96.0 & 62.2 & 64.7 & 621 \\
\hline S3-MACK-K19 & 112 & 56.5 & 8.15 & 59.7 & 0.224 & 0.09 & 0.009 & 91.1 & 52.3 & 63.1 & 727 \\
\hline S3-MACK-K20 & 80.5 & 38.2 & 7.45 & 69.0 & 0.231 & 0.10 & 0.004 & 52.9 & 50.0 & 67.0 & 622 \\
\hline S3-MACK-K21 & 49.1 & 20.7 & 3.39 & 44.2 & 0.088 & 0.12 & 0.008 & 8.27 & 51.3 & 66.6 & 381 \\
\hline S3-MACK-K22 & 31.4 & 15.8 & 6.76 & 19.9 & - & 0.11 & - & 7.14 & 9.08 & 76.4 & 259 \\
\hline S3-MACK-K23 & 12.5 & 6.73 & 4.99 & 12.8 & - & 0.13 & - & 4.25 & 5.41 & 77.8 & 168 \\
\hline S3-MACK-K24 & 66.3 & 31.5 & 8.91 & 53.5 & 0.116 & 0.09 & $\mathrm{nc}$ & 14.7 & 23.6 & 62.1 & 492 \\
\hline S3-MACK-K25 & 25.0 & 5.30 & 2.36 & 31.1 & 0.045 & 0.11 & - & 9.60 & 15.0 & 30.8 & 200 \\
\hline S3-MACK-K26 & 22.6 & 4.78 & 0.74 & 5.04 & - & 0.13 & - & 0.98 & 3.19 & 29.3 & 123 \\
\hline S3-MACK-K27 & 101 & 33.6 & 5.58 & 86.5 & 0.225 & 0.05 & 0.003 & 91.8 & 81.1 & 48.6 & 719 \\
\hline S3-MACK-K28 & 45.6 & 8.98 & 1.55 & 24.3 & 0.208 & 0.05 & 0.006 & 7.03 & 32.7 & 45.1 & 281 \\
\hline S3-MACK-K29 & 127 & 35.4 & 3.69 & 50.3 & 0.141 & 0.10 & 0.007 & 41.8 & 164 & 58.3 & 742 \\
\hline
\end{tabular}


Table 9. Results for analyses of major and minor ions, silica, and total dissolved solids (TDS) in groundwater samples collected for the Madera/Chowchilla-Kings Shallow Aquifer study unit, Groundwater Ambient Monitoring and Assessment (GAMA) Priority Basin Project, California, August 2013 to April 2014.—Continued

TThe five-digit USGS parameter code below the constituent name is used to uniquely identify a specific constituent or property. Samples from all 77 grid wells were analyzed, unless noted as not collected (nc). Information about the constituents given in table 3C. GAMA well identification numbers: S3-MACK-K, Madera/Chowchilla-Kings Shallow Aquifer Kings study-area grid well; S3-MACK-M, Madera/Chowchilla-Kings Shallow Aquifer Madera/Chowchilla study-area grid well. Benchmark type, benchmark level, and LT-MDL as of April 30, 2014. Benchmark types: Maximum contaminant level benchmarks are listed as MCL-US when the MCL-US and MCL-CA are identical, and as MCL-CA when the MCL-CA is lower than the MCL-US or no MCL-US exists. The SMCL-CA benchmarks for chloride, sulfate, and TDS are the upper benchmark levels. MCL-CA, State of California maximum contaminant level; SMCL-CA, State of California secondary maximum contaminant level.

Abbreviations: E, estimated or having a higher degree of uncertainty; LT-MDL, long-term method detection level; mg/L, milligrams per liter; na, not available; nc, not collected; SiO ${ }_{2}$, silicon dioxide; USGS, U.S. Geological Survey; - , not detected; * concentration greater than benchmark level]

\begin{tabular}{|c|c|c|c|c|c|c|c|c|c|c|c|}
\hline $\begin{array}{c}\text { GAMA } \\
\text { well } \\
\text { identification } \\
\text { number }\end{array}$ & $\begin{array}{c}\text { Calcium } \\
\text { (mg/L) } \\
(00915)\end{array}$ & $\begin{array}{c}\text { Magnesium } \\
(\mathrm{mg} / \mathrm{L}) \\
(00925)\end{array}$ & $\begin{array}{l}\text { Potassium } \\
\text { (mg/L) } \\
(00935)\end{array}$ & $\begin{array}{c}\text { Sodium } \\
(\mathrm{mg} / \mathrm{L}) \\
(00930)\end{array}$ & $\begin{array}{c}\text { Bromide } \\
\text { (mg/L) } \\
(71870)\end{array}$ & $\begin{array}{c}\text { Fluoride } \\
\text { (mg/L) } \\
(00950)\end{array}$ & $\begin{array}{c}\text { lodide } \\
\text { (mg/L) } \\
(71865)\end{array}$ & $\begin{array}{c}\text { Chloride } \\
\text { (mg/L) } \\
(00940)\end{array}$ & $\begin{array}{c}\text { Sulfate } \\
\text { (mg/L) } \\
(00945)\end{array}$ & $\begin{array}{c}\text { Silica } \\
\left.\text { (as } \mathrm{SiO}_{2}\right) \\
(\mathrm{mg} / \mathrm{L}) \\
(00955)\end{array}$ & $\begin{array}{c}\text { TDS } \\
(\mathrm{mg} / \mathrm{L}) \\
(70300)\end{array}$ \\
\hline Benchmark type & na & na & na & na & na & MCL-CA & na & SMCL-CA & SMCL-CA & na & SMCL-CA \\
\hline Benchmark level & na & na & na & na & na & 2 & na & 500 & 500 & na & 1,000 \\
\hline [LT-MDL] & {$[0.022]$} & {$[0.011]$} & {$[0.03]$} & {$[0.06]$} & {$[0.03]^{1}$} & {$[0.01]$} & {$[0.001]$} & {$[0.06]^{1}$} & {$[0.09]^{1}$} & {$[0.018]$} & [10] \\
\hline \multicolumn{12}{|c|}{ Madera/Chowchilla-Kings Shallow Aquifer study unit (77 wells sampled)_Continued } \\
\hline \multicolumn{12}{|c|}{ Kings study area (49 wells sampled)—Continued } \\
\hline S3-MACK-K30 & 18.2 & 5.92 & 1.61 & 9.39 & - & 0.18 & - & 1.91 & 4.85 & 55.1 & 161 \\
\hline S3-MACK-K31 & 16.6 & 5.35 & 2.12 & 19.9 & 0.057 & 0.13 & - & 14.0 & 4.52 & 46.3 & 156 \\
\hline S3-MACK-K32 & 14.6 & 3.91 & 1.67 & 7.46 & - & 0.08 & - & 2.76 & 7.74 & 34.8 & 113 \\
\hline S3-MACK-K33 & 77.2 & 41.7 & 3.47 & 20.6 & 0.190 & 0.09 & $\mathrm{nc}$ & 30.8 & 36.6 & 55.4 & 489 \\
\hline S3-MACK-K34 & 54.3 & 34.4 & 10.2 & 38.2 & 0.096 & 0.10 & $\mathrm{nc}$ & 21.8 & 29.7 & 73.1 & 451 \\
\hline S3-MACK-K35 & 17.2 & 8.56 & 2.44 & 11.7 & 0.038 & 0.14 & - & 5.46 & 6.91 & 57.8 & 158 \\
\hline S3-MACK-K36 & 33.1 & 14.3 & 3.36 & 19.9 & 0.039 & 0.12 & - & 7.58 & 12.6 & 58.1 & 248 \\
\hline S3-MACK-K37 & 15.5 & 8.91 & 3.00 & 13.6 & - & 0.10 & 0.001 & 4.48 & 3.67 & 60.5 & 163 \\
\hline S3-MACK-K38 & 74.3 & 33.4 & 3.26 & 38.9 & 0.266 & 0.08 & $\mathrm{nc}$ & 21.3 & 123 & 70.0 & 560 \\
\hline S3-MACK-K39 & 70.9 & 19.6 & 4.96 & 35.4 & 0.122 & 0.05 & 0.001 & 27.4 & 51.9 & 32.0 & 404 \\
\hline S3-MACK-K40 & 98.8 & 34.5 & 5.15 & 69.4 & 0.281 & 0.06 & 0.006 & 33.1 & 96.0 & 50.9 & 680 \\
\hline S3-MACK-K41 & 99.7 & 34.4 & 3.91 & 39.1 & 0.231 & 0.12 & 0.003 & 45.9 & 61.8 & 51.4 & 573 \\
\hline S3-MACK-K42 & 49.5 & 42.7 & 2.16 & 76.4 & 0.149 & 0.17 & 0.006 & 45.6 & 74.1 & 59.5 & 589 \\
\hline S3-MACK-K43 & 96.7 & 44.6 & 4.41 & 41.0 & 0.098 & 0.12 & 0.004 & 32.8 & 56.8 & 55.8 & 622 \\
\hline S3-MACK-K44 & 114 & 37.5 & 5.72 & 61.2 & 0.302 & 0.16 & 0.007 & 67.7 & 72.3 & 51.5 & 711 \\
\hline S3-MACK-K45 & 52.0 & 21.2 & 3.31 & 54.5 & 0.05 & 0.22 & 0.004 & 9.75 & 12.4 & 53.6 & 429 \\
\hline S3-MACK-K46 & 52.7 & 18.2 & 2.83 & 15.6 & - & 0.02 & 0.011 & 2.08 & 8.10 & 37.0 & 265 \\
\hline S3-MACK-K47 & 122 & 42.9 & 3.12 & 48.1 & 0.255 & 0.08 & 0.010 & 144 & 98.6 & 46.6 & 684 \\
\hline S3-MACK-K48 & 53.3 & 23.0 & 3.71 & 24.8 & 0.106 & 0.13 & 0.002 & 26.0 & 16.9 & 49.8 & 336 \\
\hline
\end{tabular}


Table 9. Results for analyses of major and minor ions, silica, and total dissolved solids (TDS) in groundwater samples collected for the Madera/Chowchilla-Kings Shallow Aquifer study unit, Groundwater Ambient Monitoring and Assessment (GAMA) Priority Basin Project, California, August 2013 to April 2014.—Continued

[The five-digit USGS parameter code below the constituent name is used to uniquely identify a specific constituent or property. Samples from all 77 grid wells were analyzed, unless noted as not collected (nc). Information about the constituents given in table 3C. GAMA well identification numbers: S3-MACK-K, Madera/Chowchilla-Kings Shallow Aquifer Kings study-area grid well; S3-MACK-M, Madera/Chowchilla-Kings Shallow Aquifer Madera/Chowchilla study-area grid well. Benchmark type, benchmark level, and LT-MDL as of April 30, 2014. Benchmark types: Maximum contaminant level benchmarks are listed as MCL-US when the MCL-US and MCL-CA are identical, and as MCL-CA when the MCL-CA is lower than the MCL-US or no MCL-US exists. The SMCL-CA benchmarks for chloride, sulfate, and TDS are the upper benchmark levels. MCL-CA, State of California maximum contaminant level; SMCL-CA, State of California secondary maximum contaminant level. Abbreviations: E, estimated or having a higher degree of uncertainty; $\mathrm{LT}-\mathrm{MDL}$, long-term method detection level; $\mathrm{mg} / \mathrm{L}$, milligrams per liter; na, not available; nc, not collected; $\mathrm{SiO}_{2}$, silicon dioxide USGS, U.S. Geological Survey; - , not detected; * concentration greater than benchmark level]

\begin{tabular}{|c|c|c|c|c|c|c|c|c|c|c|c|}
\hline $\begin{array}{c}\text { GAMA } \\
\text { well } \\
\text { identification } \\
\text { number }\end{array}$ & $\begin{array}{c}\text { Calcium } \\
\text { (mg/L) } \\
(00915)\end{array}$ & $\begin{array}{c}\text { Magnesium } \\
(\mathrm{mg} / \mathrm{L}) \\
(00925)\end{array}$ & $\begin{array}{c}\text { Potassium } \\
\text { (mg/L) } \\
(00935)\end{array}$ & $\begin{array}{c}\text { Sodium } \\
\text { (mg/L) } \\
(00930)\end{array}$ & $\begin{array}{c}\text { Bromide } \\
\text { (mg/L) } \\
(71870)\end{array}$ & $\begin{array}{c}\text { Fluoride } \\
\text { (mg/L) } \\
(00950)\end{array}$ & $\begin{array}{l}\text { lodide } \\
\text { (mg/L) } \\
(71865)\end{array}$ & $\begin{array}{c}\text { Chloride } \\
\text { (mg/L) } \\
(00940)\end{array}$ & $\begin{array}{l}\text { Sulfate } \\
\text { (mg/L) } \\
(00945)\end{array}$ & $\begin{array}{c}\text { Silica } \\
\text { (as SiO }) \\
(\mathrm{mg} / \mathrm{L}) \\
(00955)\end{array}$ & $\begin{array}{c}\text { TDS } \\
(\mathrm{mg} / \mathrm{L}) \\
(70300)\end{array}$ \\
\hline Benchmark type & na & na & na & na & na & MCL-CA & na & SMCL-CA & SMCL-CA & na & SMCL-CA \\
\hline Benchmark level & na & na & na & na & na & 2 & na & 500 & 500 & na & 1,000 \\
\hline [LT-MDL] & {$[0.022]$} & {$[0.011]$} & {$[0.03]$} & {$[0.06]$} & {$[0.03]^{1}$} & {$[0.01]$} & {$[0.001]$} & {$[0.06]^{1}$} & {$[0.09]^{1}$} & {$[0.018]$} & [10] \\
\hline \multicolumn{12}{|c|}{ Madera/Chowchilla-Kings Shallow Aquifer study unit (77 wells sampled)_Continued } \\
\hline \multicolumn{12}{|c|}{ Kings study area (49 wells sampled)—Continued } \\
\hline S3-MACK-K49 & 84.2 & 28.2 & 4.34 & 27.8 & 0.076 & 0.11 & 0.007 & 30.9 & 42.0 & 44.2 & 446 \\
\hline
\end{tabular}

${ }^{1}$ The LT-MDL changed during the period that samples were collected for this study (see table $3 C$ ). The higher of the two LT-MDLs is reported here, and detections at concentrations less than this LT-MDL were replaced with a - symbol, indicating a non-detection relative to the higher LT-MDL.

${ }^{2}$ The USGS Branch of Quality Systems (BQS) Inorganic Blind Sample Project (IBSP) fluoride data show a positive bias. If the bias observed in the IBSP samples can be assumed to apply to concentration outside of the concentration range of the IBSP samples, then the two MACK study unit samples that had measured concentrations just over one-half the value of the MCL-CA for fluoride could have actual concentrations just less than one-half the MCL-CA (S3-MACK-K02, $1.05 \mathrm{mg} / \mathrm{L}$; S3-MACK-K13, $1.07 \mathrm{mg} / \mathrm{L}$ ).

${ }^{3}$ The laboratory raised the reporting limit from $0.01 \mu \mathrm{g} / \mathrm{L}$ to $0.10 \mu \mathrm{g} / \mathrm{L}$. 
Table 10. Results for analyses of perchlorate in groundwater samples collected for the Madera/Chowchilla-Kings Shallow Aquifer study unit, Groundwater Ambient Monitoring and Assessment (GAMA) Priority Basin Project, California, August 2013 to April 2014.

[The five-digit USGS parameter code below the constituent name is used to uniquely identify a specific constituent or property. Samples from 76 grid wells were analyzed, the sample from -K24 was frozen and could not be analyzed. Information about the constituent is given in table 3C. GAMA well identification numbers: S3-MACK-K, Madera/Chowchilla-Kings Shallow Aquifer Kings study-area grid well; S3-MACK-M, Madera/Chowchilla-Kings Shallow Aquifer Madera/Chowchilla study-area grid well. Abbreviations: MCL-CA, State of California maximum contaminant level; MRL, method reporting level; nc, not collected; USGS, U.S. Geological Survey; $\mu \mathrm{g} / \mathrm{L}$, micrograms per liter; - , not detected]

\begin{tabular}{lc}
\hline $\begin{array}{c}\text { GAMA well } \\
\text { identification } \\
\text { number }\end{array}$ & $\begin{array}{c}\text { Perchlorate } \\
(\mu \mathrm{g} / \mathrm{L}) \\
(63790)\end{array}$ \\
\hline Benchmark type & MCL-CA \\
Benchmark level & 6 \\
{$[\mathrm{MRL}]$} & {$[0.10]$} \\
\hline
\end{tabular}

Madera/Chowchilla-Kings shallow aquifer study area (76 wells sampled)

Madera/Chowchilla study area (28 wells sampled)

\begin{tabular}{|c|c|}
\hline S3-MACK-M01 & - \\
\hline S3-MACK-M02 & 0.12 \\
\hline S3-MACK-M03 & 0.25 \\
\hline S3-MACK-M04 & - \\
\hline S3-MACK-M05 & 0.39 \\
\hline S3-MACK-M06 & 0.50 \\
\hline S3-MACK-M07 & 0.28 \\
\hline S3-MACK-M08 & 0.80 \\
\hline S3-MACK-M09 & 1.12 \\
\hline S3-MACK-M10 & - $^{(1)}$ \\
\hline S3-MACK-M11 & 0.33 \\
\hline S3-MACK-M12 & 0.84 \\
\hline S3-MACK-M13 & 0.15 \\
\hline S3-MACK-M14 & 0.48 \\
\hline S3-MACK-M15 & - $^{(1)}$ \\
\hline S3-MACK-M16 & 0.58 \\
\hline S3-MACK-M17 & 0.99 \\
\hline S3-MACK-M18 & 0.38 \\
\hline S3-MACK-M19 & 0.45 \\
\hline S3-MACK-M20 & 0.24 \\
\hline S3-MACK-M21 & - $^{(1)}$ \\
\hline S3-MACK-M22 & 1.12 \\
\hline S3-MACK-M23 & - \\
\hline S3-MACK-M24 & 0.46 \\
\hline S3-MACK-M25 & 0.57 \\
\hline S3-MACK-M26 & 0.91 \\
\hline S3-MACK-M27 & 0.36 \\
\hline S3-MACK-M28 & - \\
\hline
\end{tabular}

\begin{tabular}{lc}
\hline $\begin{array}{c}\text { GAMA well } \\
\text { identification } \\
\text { number }\end{array}$ & $\begin{array}{c}\text { Perchlorate } \\
(\mu \mathrm{g} / \mathrm{L})\end{array}$ \\
\hline Benchmark type & $(63790)$ \\
Benchmark level & MCL-CA \\
{$[\mathrm{MRL}]$} & 6 \\
\hline
\end{tabular}

Madera/Chowchilla-Kings shallow aquifer study area (76 wells sampled) - Continued

Kings study area (48 samples)

S3-MACK-K01

S3-MACK-K02

S3-MACK-K03

S3-MACK-K04

S3-MACK-K05

S3-MACK-K06

S3-MACK-K07

S3-MACK-K08

0.12

S3-MACK-K09

S3-MACK-K10

4.26

S3-MACK-K11

1.37

S3-MACK-K12

S3-MACK-K13

S3-MACK-K14

S3-MACK-K15

S3-MACK-K16

S3-MACK-K17

S3-MACK-K18

S3-MACK-K19

S3-MACK-K20

S3-MACK-K21

0.14

S3-MACK-K22

0.40

S3-MACK-K23

0.20

S3-MACK-K24

$\mathrm{nc}$

S3-MACK-K25 0.35

S3-MACK-K26

0.12

S3-MACK-K27

2.21

S3-MACK-K28

0.50

S3-MACK-K29 
Table 10. Results for analyses of perchlorate in groundwater samples collected for the Madera/Chowchilla-Kings Shallow Aquifer study unit, Groundwater Ambient Monitoring and Assessment (GAMA) Priority Basin Project, California, August 2013 to April 2014.-Continued

[The five-digit USGS parameter code below the constituent name is used to uniquely identify a specific constituent or property. Samples from 76 grid wells were analyzed, the sample from -K24 was frozen and could not be analyzed. Information about the constituent is given in table 3C. GAMA well identification numbers: S3-MACK-K, Madera/Chowchilla-Kings Shallow Aquifer Kings study-area grid well; S3-MACK-M, Madera/ Chowchilla-Kings Shallow Aquifer Madera/Chowchilla study-area grid well. Abbreviations: MCL-CA, State of California maximum contaminant level; MRL, method reporting level; nc, not collected; USGS, U.S. Geological Survey; $\mu \mathrm{g} / \mathrm{L}$, micrograms per liter; —, not detected]

\begin{tabular}{|c|c|}
\hline $\begin{array}{l}\text { GAMA well } \\
\text { identification } \\
\text { number }\end{array}$ & $\begin{array}{c}\text { Perchlorate } \\
(\mu \mathrm{g} / \mathrm{L}) \\
(63790)\end{array}$ \\
\hline Benchmark type & MCL-CA \\
\hline Benchmark level & 6 \\
\hline [MRL] & [0.10] \\
\hline \multicolumn{2}{|c|}{$\begin{array}{l}\text { Madera/Chowchilla-Kings shallow aquifer study area } \\
\text { (76 wells sampled)_Continued }\end{array}$} \\
\hline \multicolumn{2}{|c|}{ Kings study area (48 samples)—Continued } \\
\hline S3-MACK-K30 & 0.24 \\
\hline S3-MACK-K31 & 0.44 \\
\hline S3-MACK-K32 & 0.19 \\
\hline S3-MACK-K33 & 2.13 \\
\hline S3-MACK-K34 & 2.58 \\
\hline S3-MACK-K35 & 0.41 \\
\hline S3-MACK-K36 & 0.78 \\
\hline S3-MACK-K37 & - \\
\hline S3-MACK-K38 & 2.32 \\
\hline S3-MACK-K39 & 2.76 \\
\hline S3-MACK-K40 & 2.49 \\
\hline S3-MACK-K41 & 2.44 \\
\hline S3-MACK-K42 & 0.77 \\
\hline S3-MACK-K43 & 3.05 \\
\hline S3-MACK-K44 & 3.62 \\
\hline S3-MACK-K45 & 1.11 \\
\hline S3-MACK-K46 & - \\
\hline S3-MACK-K47 & 0.70 \\
\hline S3-MACK-K48 & 0.42 \\
\hline S3-MACK-K49 & 1.65 \\
\hline
\end{tabular}

${ }^{1}$ The laboratory raised the reporting level from $0.10 \mu \mathrm{g} / \mathrm{L}$ to $0.76 \mu \mathrm{g} / \mathrm{L}$. 
Table 11. Results for analyses of radioactive constituents in groundwater samples collected for the Madera/Chowchilla-Kings Shallow Aquifer study unit, Groundwater Ambient Monitoring and Assessment (GAMA) Priority Basin Project, California, August 2013 to April 2014.

[The five-digit USGS parameter code below the constituent name is used to uniquely identify a specific constituent or property. Samples from all 77 grid wells were analyzed, unless noted as not collected (nc). Information about the constituents given in table 3C. Measured values less than the sample-specific critical level ( $\mathrm{ssL}_{\mathrm{C}}$ ) are reported as non-detections (-). GAMA well identification numbers: S3-MACK-K, Madera/Chowchilla-Kings Shallow Aquifer Kings study-area grid well; S3-MACK-M, Madera/Chowchilla-Kings Shallow Aquifer Madera/Chowchilla study-area grid well. Benchmark type and benchmark level as of April 30, 2014. Benchmark types: Maximum contaminant level benchmarks are listed as MCL-US when the MCL-US and MCL-CA are identical, and as MCL-CA when the MCL-CA is lower than the MCL-US or no MCL-US exists. MCL-CA, State of California maximum contaminant level; MCL-US, U.S. Environmental Protection Agency maximum contaminant level. Abbreviations: CSU, 1-sigma combined standard uncertainty; pCi/L, picocuries per liter; RL, reporting level; USGS, U.S. Geological Survey; —, not detected; \pm , plus or minus; *, activity greater than the benchmark level]

\begin{tabular}{|c|c|c|c|c|c|c|c|c|c|c|c|c|}
\hline $\begin{array}{c}\text { GAMA well } \\
\text { identification } \\
\text { number }\end{array}$ & \multicolumn{2}{|c|}{$\begin{array}{c}\text { Radon-222 } \\
(\mathrm{pCi} / \mathrm{L}) \\
(82303)\end{array}$} & \multicolumn{2}{|c|}{$\begin{array}{c}\text { Gross alpha radioactivity, } \\
\text { 72-hour count } \\
\text { (pCi/L) } \\
(62636)^{1}\end{array}$} & \multicolumn{2}{|c|}{$\begin{array}{c}\text { Gross alpha radioactivity, } \\
\text { 30-day count } \\
\text { (pCi/L) } \\
(62639)\end{array}$} & \multicolumn{2}{|c|}{$\begin{array}{c}\text { Gross beta radioactivity, } \\
\text { 72-hour count } \\
\text { (pCi/L) } \\
(62642)^{1}\end{array}$} & \multicolumn{2}{|c|}{$\begin{array}{c}\text { Gross beta radioactivity, } \\
\text { 30-day count } \\
\text { (pCi/L) } \\
(62645)\end{array}$} & \multicolumn{2}{|c|}{$\begin{array}{l}\text { Tritium } \\
\text { (pCi/L) } \\
\text { (07000) }\end{array}$} \\
\hline Benchmark type & \multicolumn{2}{|c|}{ Proposed MCL-US } & \multicolumn{2}{|c|}{ MCL-US ${ }^{2}$} & \multicolumn{2}{|c|}{ MCL-US ${ }^{2}$} & \multicolumn{2}{|c|}{ MCL-CA } & \multicolumn{2}{|c|}{ MCL-CA } & \multicolumn{2}{|c|}{ MCL-CA } \\
\hline Benchmark level & \multicolumn{2}{|l|}{4,000} & \multicolumn{2}{|c|}{15} & \multicolumn{2}{|c|}{15} & \multicolumn{2}{|l|}{50} & \multicolumn{2}{|l|}{50} & \multicolumn{2}{|l|}{20,000} \\
\hline [RL] & Result \pm CSU & ssL $_{c}$ & Result \pm CSU & $\mathbf{s s L}_{\mathrm{c}}$ & Result \pm CSU & $\mathrm{ssL}_{\mathrm{c}}$ & Result \pm CSU & $\mathbf{s s L}_{\mathrm{c}}$ & Result \pm CSU & ssL $_{c}$ & Result \pm CSU & ssL $_{c}$ \\
\hline \multicolumn{13}{|c|}{ Madera/Chowchilla-Kings Shallow Aquifer study unit (73 to 77 wells sampled) } \\
\hline \multicolumn{13}{|c|}{ Madera/Chowchilla study area (28 wells sampled) } \\
\hline S3-MACK-M01 & $908 \pm 52$ & 11 & $0.76 \pm 0.42$ & 0.62 & - \pm 0.31 & 0.60 & $4.77 \pm 0.90$ & 1.3 & $4.63 \pm 0.86$ & 1.2 & $10.3 \pm 0.46$ & 0.32 \\
\hline S3-MACK-M02 & $491 \pm 30$ & 11 & $2.27 \pm 0.93$ & 1.3 & $3.03 \pm 0.91$ & 1.1 & $5.65 \pm 0.88$ & 1.1 & $5.88 \pm 0.89$ & 1.1 & - \pm 0.80 & 0.52 \\
\hline S3-MACK-M03 & $670 \pm 42$ & 17 & ${ }^{1} 48.8 \pm 7.6$ & 4.8 & $37.0 \pm 6.9$ & 5.9 & ${ }^{1} 16.9 \pm 2.7$ & 3.4 & $33.1 \pm 3.9$ & 3.6 & $5.0 \pm 0.80$ & 0.52 \\
\hline S3-MACK-M04 & $504 \pm 34$ & 17 & ${ }^{1} 23.5 \pm 3.5$ & 2.1 & $22.8 \pm 3.5$ & 2.3 & ${ }^{1} 8.5 \pm 1.4$ & 1.8 & $18.0 \pm 2.1$ & 1.8 & $2.9 \pm 0.63$ & 0.20 \\
\hline S3-MACK-M05 & $661 \pm 40$ & 14 & ${ }^{1} 24.1 \pm 3.8$ & 1.8 & $28.8 \pm 4.4$ & 2.1 & ${ }^{1} 13.3 \pm 1.8$ & 2.0 & $17.3 \pm 2.0$ & 2.0 & $8.2 \pm 0.59$ & 0.49 \\
\hline S3-MACK-M06 & $580 \pm 35$ & 11 & $4.6 \pm 1.4$ & 1.8 & $5.6 \pm 1.6$ & 1.9 & $10.1 \pm 1.3$ & 1.4 & $8.5 \pm 1.3$ & 1.5 & $3.5 \pm 0.28$ & 0.28 \\
\hline S3-MACK-M07 & $1,228 \pm 69$ & 11 & $4.4 \pm 1.0$ & 1.1 & $2.79 \pm 0.66$ & 0.59 & $6.6 \pm 1.1$ & 1.4 & $7.2 \pm 1.1$ & 1.4 & $9.3 \pm 0.59$ & 0.49 \\
\hline S3-MACK-M08 & $534 \pm 33$ & 14 & ${ }^{1} 4.6 \pm 1.5$ & 1.8 & $9.7 \pm 1.9$ & 1.4 & ${ }^{1} 7.7 \pm 1.2$ & 1.6 & $9.5 \pm 1.5$ & 1.8 & $12.7 \pm 0.59$ & 0.49 \\
\hline S3-MACK-M09 & $240 \pm 18$ & 12 & ${ }^{1} 4.9 \pm 1.3$ & 1.5 & $2.2 \pm 1.2$ & 1.8 & ${ }^{1} 8.5 \pm 1.2$ & 1.5 & $8.6 \pm 1.3$ & 1.5 & $11.9 \pm 0.26$ & 0.26 \\
\hline S3-MACK-M10 & $375 \pm 24$ & 11 & $18.6 \pm 3.1$ & 2.2 & $18.6 \pm 3.2$ & 2.2 & $15.3 \pm 1.8$ & 1.7 & $23.2 \pm 2.4$ & 1.5 & $5.5 \pm 0.80$ & 0.52 \\
\hline S3-MACK-M11 & $611 \pm 37$ & 12 & $34.5 \pm 4.5$ & 1.5 & $27.4 \pm 3.7$ & 1.3 & $7.0 \pm 1.1$ & 1.4 & $25.9 \pm 2.7$ & 1.3 & $19.2 \pm 0.90$ & 0.49 \\
\hline S3-MACK-M12 & $224 \pm 17$ & 11 & $11.4 \pm 2.0$ & 1.6 & $10.0 \pm 1.8$ & 1.5 & $3.49 \pm 0.86$ & 1.3 & $7.4 \pm 1.1$ & 1.3 & $10.6 \pm 0.46$ & 0.32 \\
\hline S3-MACK-M13 & $321 \pm 23$ & 14 & $0.70 \pm 0.42$ & 0.65 & $0.96 \pm 0.47$ & 0.70 & $6.4 \pm 1.0$ & 1.3 & $6.6 \pm 1.0$ & 1.3 & $14.1 \pm 0.80$ & 0.52 \\
\hline S3-MACK-M14 & $891 \pm 51$ & 11 & - \pm 0.53 & 0.89 & $1.17 \pm 0.61$ & 0.93 & $4.13 \pm 0.89$ & 1.3 & $3.85 \pm 0.83$ & 1.2 & $18.2 \pm 0.63$ & 0.20 \\
\hline S3-MACK-M15 & $594 \pm 36$ & 12 & $2.19 \pm 0.76$ & 1.0 & $1.51 \pm 0.63$ & 0.88 & $8.5 \pm 1.2$ & 1.4 & $5.96 \pm 0.99$ & 1.3 & $15.1 \pm 0.21$ & 0.20 \\
\hline S3-MACK-M16 & $1,190 \pm 68$ & 13 & $13.1 \pm 2.7$ & 2.5 & $15.8 \pm 2.9$ & 2.4 & $10.2 \pm 1.4$ & 1.6 & $16.8 \pm 2.0$ & 1.8 & $9.3 \pm 0.59$ & 0.49 \\
\hline S3-MACK-M17 & $124 \pm 11$ & 11 & $19.4 \pm 3.5$ & 2.9 & $15.9 \pm 3.2$ & 3.0 & $9.3 \pm 1.8$ & 2.6 & $20.6 \pm 2.5$ & 2.3 & $8.7 \pm 0.28$ & 0.28 \\
\hline S3-MACK-M18 & $334 \pm 23$ & 13 & - \pm 0.64 & 1.1 & - \pm 0.53 & 0.91 & $3.56 \pm 0.90$ & 1.4 & $3.11 \pm 0.87$ & 1.4 & $1.4 \pm 0.59$ & 0.49 \\
\hline S3-MACK-M19 & $297 \pm 20$ & 11 & - \pm 0.57 & 0.98 & - \pm 0.50 & 0.85 & $5.12 \pm 0.95$ & 1.3 & $5.37 \pm 0.96$ & 1.3 & $1.7 \pm 0.21$ & 0.20 \\
\hline
\end{tabular}


Table 11. Results for analyses of radioactive constituents in groundwater samples collected for the Madera/Chowchilla-Kings Shallow Aquifer study unit, Groundwater Ambient Monitoring and Assessment (GAMA) Priority Basin Project, California, August 2013 to April 2014.-Continued

[The five-digit USGS parameter code below the constituent name is used to uniquely identify a specific constituent or property. Samples from all 77 grid wells were analyzed, unless noted as not collected (nc). Information about the constituents given in table $3 C$. Measured values less than the sample-specific critical level ( $\mathrm{ssL}_{\mathrm{C}}$ ) are reported as non-detections (-). GAMA well identification numbers: S3-MACK-K, Madera/Chowchilla-Kings Shallow Aquifer Kings study-area grid well; S3-MACK-M, Madera/Chowchilla-Kings Shallow Aquifer Madera/Chowchilla study-area grid well. Benchmark type and benchmark level as of April 30, 2014. Benchmark types: Maximum contaminant level benchmarks are listed as MCL-US when the MCL-US and MCL-CA are identical, and as MCL-CA when the MCL-CA is lower than the MCL-US or no MCL-US exists. MCL-CA, State of California maximum contaminant level; MCL-US, U.S. Environmental Protection Agency maximum contaminant level. Abbreviations: CSU, 1-sigma combined standard uncertainty; pCi/L, picocuries per liter; RL, reporting level; USGS, U.S. Geological Survey; —, not detected; \pm , plus or minus; *, activity greater than the benchmark level]

\begin{tabular}{|c|c|c|c|c|c|c|c|c|c|c|c|c|}
\hline \multirow{4}{*}{\begin{tabular}{l}
\multicolumn{1}{c}{$\begin{array}{c}\text { GAMA well } \\
\text { identification } \\
\text { number }\end{array}$} \\
Benchmark type \\
Benchmark level \\
[RL]
\end{tabular}} & \multicolumn{2}{|c|}{$\begin{array}{c}\text { Radon-222 } \\
(\mathrm{pCi} / \mathrm{L}) \\
(82303)\end{array}$} & \multicolumn{2}{|c|}{$\begin{array}{c}\text { Gross alpha radioactivity, } \\
\text { 72-hour count } \\
\text { (pCi/L) } \\
(62636)^{1}\end{array}$} & \multicolumn{2}{|c|}{$\begin{array}{c}\text { Gross alpha radioactivity, } \\
\text { 30-day count } \\
\text { (pCi/L) } \\
(62639)\end{array}$} & \multicolumn{2}{|c|}{$\begin{array}{c}\text { Gross beta radioactivity, } \\
\text { 72-hour count } \\
\text { (pCi/L) } \\
(62642)^{1}\end{array}$} & \multicolumn{2}{|c|}{$\begin{array}{c}\text { Gross beta radioactivity, } \\
\text { 30-day count } \\
\text { (pCi/L) } \\
(62645)\end{array}$} & \multicolumn{2}{|c|}{$\begin{array}{l}\text { Tritium } \\
\text { (pCi/L) } \\
\text { (07000) }\end{array}$} \\
\hline & \multicolumn{2}{|c|}{ Proposed MCL-US } & \multicolumn{2}{|c|}{ MCL-US ${ }^{2}$} & \multicolumn{2}{|c|}{ MCL-US ${ }^{2}$} & \multicolumn{2}{|c|}{ MCL-CA } & \multicolumn{2}{|c|}{ MCL-CA } & \multicolumn{2}{|c|}{ MCL-CA } \\
\hline & \multicolumn{2}{|l|}{4,000} & \multicolumn{2}{|c|}{15} & \multicolumn{2}{|l|}{15} & \multicolumn{2}{|l|}{50} & \multicolumn{2}{|l|}{50} & \multicolumn{2}{|l|}{20,000} \\
\hline & Result \pm CSU & ssL $_{c}$ & Result \pm CSU & $\mathbf{s s L}_{\mathrm{c}}$ & Result \pm CSU & ssL $_{\mathrm{c}}$ & Result \pm CSU & ssL $_{c}$ & Result \pm CSU & ssL $_{c}$ & Result \pm CSU & ssL $_{\mathrm{c}}$ \\
\hline \multicolumn{13}{|c|}{ Madera/Chowchilla-Kings Shallow Aquifer study unit (73 to 77 wells sampled)_Continued } \\
\hline \multicolumn{13}{|c|}{ Madera/Chowchilla study area (28 wells sampled)_Continued } \\
\hline S3-MACK-M20 & $341 \pm 22$ & 11 & - \pm 0.49 & 0.84 & - \pm 0.43 & 0.80 & $5.37 \pm 0.95$ & 1.3 & $1.7 \pm 0.70$ & 1.2 & $1.9 \pm 0.56$ & 0.53 \\
\hline S3-MACK-M21 & $680 \pm 40$ & 12 & $3.8 \pm 1.0$ & 1.2 & $6.7 \pm 1.3$ & 1.2 & $7.1 \pm 1.1$ & 1.5 & $7.4 \pm 1.1$ & 1.4 & $3.0 \pm 0.8$ & 0.52 \\
\hline S3-MACK-M22 & $624 \pm 38$ & 14 & - \pm 0.75 & 1.3 & $1.59 \pm 0.81$ & 1.2 & $6.0 \pm 1.0$ & 1.4 & $5.27 \pm 0.97$ & 1.3 & $4.4 \pm 0.51$ & 0.26 \\
\hline S3-MACK-M23 & $1,582 \pm 89$ & 14 & - \pm 0.36 & 0.62 & - \pm 0.40 & 0.70 & $5.69 \pm 0.95$ & 1.3 & $2.71 \pm 0.76$ & 1.2 & $9.3 \pm 0.51$ & 0.26 \\
\hline S3-MACK-M24 & $784 \pm 47$ & 14 & $1.95 \pm 0.64$ & 0.85 & - \pm 0.42 & 0.75 & $3.64 \pm 0.79$ & 1.2 & $3.82 \pm 0.87$ & 1.3 & - \pm 0.8 & 0.52 \\
\hline S3-MACK-M25 & $1,528 \pm 85$ & 12 & - \pm 0.62 & 0.98 & - \pm 0.50 & 0.77 & $3.35 \pm 0.81$ & 1.2 & $1.97 \pm 0.74$ & 1.2 & - \pm 0.63 & 0.20 \\
\hline S3-MACK-M26 & $306 \pm 21$ & 11 & ${ }^{1}- \pm 0.58$ & 0.98 & - \pm 0.64 & 1.1 & ${ }^{1} 4.61 \pm 0.94$ & 1.3 & $3.57 \pm 0.97$ & 1.5 & $0.8 \pm 0.21$ & 0.20 \\
\hline S3-MACK-M27 & $325 \pm 22$ & 12 & ${ }^{1}- \pm 0.49$ & 0.96 & - \pm 0.59 & 0.98 & ${ }^{1} 6.63 \pm 0.97$ & 1.1 & $2.57 \pm 0.79$ & 1.3 & $5.8 \pm 0.59$ & 0.49 \\
\hline S3-MACK-M28 & $344 \pm 23$ & 13 & - \pm 1.0 & 1.6 & $1.48 \pm 0.87$ & 1.3 & $4.6 \pm 1.1$ & 1.7 & $6.5 \pm 1.2$ & 1.6 & - \pm 0.28 & 0.28 \\
\hline \multicolumn{13}{|c|}{ Kings study area ( 45 to 49 wells sampled) } \\
\hline S3-MACK-K01 & $469 \pm 30$ & 14 & $\mathrm{nc}$ & $\mathrm{nc}$ & $* 122 \pm 14$ & 0.67 & $20.4 \pm 2.9$ & 3.5 & $* 99.2 \pm 9.1$ & 2.9 & $9.3 \pm 0.41$ & 0.21 \\
\hline S3-MACK-K02 & $712 \pm 43$ & 13 & - \pm 3.1 & 5.2 & - \pm 3.1 & 5.3 & $5.2 \pm 1.7$ & 2.8 & - \pm 1.6 & 2.9 & $16.3 \pm 0.61$ & 0.23 \\
\hline S3-MACK-K03 & $1,322 \pm 74$ & 11 & $\mathrm{nc}$ & nc & $\mathrm{nc}$ & nc & nc & nc & nc & $\mathrm{nc}$ & $1.7 \pm 0.45$ & 0.42 \\
\hline S3-MACK-K04 & $1,020 \pm 59$ & 13 & $* 235 \pm 28$ & 3.0 & $200 \pm 23$ & 1.7 & ${ }^{1} 45.0 \pm 4.1$ & 1.6 & $* 147 \pm 13$ & 1.6 & $2.2 \pm 0.45$ & 0.42 \\
\hline S3-MACK-K05 & $* 6,720 \pm 360$ & 11 & ${ }^{1} 112 \pm 14$ & 2.1 & $102 \pm 13$ & 2.5 & ${ }^{1} 13.0 \pm 1.5$ & 1.4 & $* 50.5 \pm 4.7$ & 1.6 & $0.8 \pm 0.62$ & 0.39 \\
\hline S3-MACK-K06 & $543 \pm 33$ & 11 & nc & $\mathrm{nc}$ & $\mathrm{nc}$ & $\mathrm{nc}$ & $\mathrm{nc}$ & $\mathrm{nc}$ & $\mathrm{nc}$ & $\mathrm{nc}$ & $2.4 \pm 0.62$ & 0.39 \\
\hline S3-MACK-K07 & $686 \pm 40$ & 10 & $11.9 \pm 3.7$ & 4.7 & $8.9 \pm 3.1$ & 4.0 & - \pm 1.6 & 2.8 & $7.9 \pm 1.8$ & 2.6 & $1.7 \pm 0.62$ & 0.39 \\
\hline S3-MACK-K08 & $785 \pm 46$ & 11 & nc & $\mathrm{nc}$ & $\mathrm{nc}$ & $\mathrm{nc}$ & $\mathrm{nc}$ & $\mathrm{nc}$ & $\mathrm{nc}$ & $\mathrm{nc}$ & $13.8 \pm 0.49$ & 0.30 \\
\hline S3-MACK-K09 & $662 \pm 40$ & 13 & $3.5 \pm 2.0$ & 3.1 & - \pm 2.2 & 3.8 & $6.4 \pm 1.7$ & 2.6 & $4.1 \pm 1.5$ & 2.6 & - \pm 0.46 & 0.33 \\
\hline S3-MACK-K10 & $416 \pm 27$ & 14 & $123 \pm 16$ & 6.2 & $* 124 \pm 16$ & 4.4 & ${ }^{1} 45.0 \pm 5.1$ & 4.3 & $* 94.2 \pm 8.5$ & 3.3 & $1.7 \pm 0.62$ & 0.39 \\
\hline
\end{tabular}


Table 11. Results for analyses of radioactive constituents in groundwater samples collected for the Madera/Chowchilla-Kings Shallow Aquifer study unit, Groundwater Ambient Monitoring and Assessment (GAMA) Priority Basin Project, California, August 2013 to April 2014.-Continued

[The five-digit USGS parameter code below the constituent name is used to uniquely identify a specific constituent or property. Samples from all 77 grid wells were analyzed, unless noted as not collected (nc). Information about the constituents given in table $3 C$. Measured values less than the sample-specific critical level ( $\mathrm{ssL}_{\mathrm{C}}$ ) are reported as non-detections (-). GAMA well identification numbers: S3-MACK-K, Madera/Chowchilla-Kings Shallow Aquifer Kings study-area grid well; S3-MACK-M, Madera/Chowchilla-Kings Shallow Aquifer Madera/Chowchilla study-area grid well. Benchmark type and benchmark level as of April 30, 2014. Benchmark types: Maximum contaminant level benchmarks are listed as MCL-US when the MCL-US and MCL-CA are identical, and as MCL-CA when the MCL-CA is lower than the MCL-US or no MCL-US exists. MCL-CA, State of California maximum contaminant level; MCL-US, U.S. Environmental Protection Agency maximum contaminant level. Abbreviations: CSU, 1-sigma combined standard uncertainty; pCi/L, picocuries per liter; RL, reporting level; USGS, U.S. Geological Survey; —, not detected; \pm , plus or minus; *, activity greater than the benchmark level]

\begin{tabular}{|c|c|c|c|c|c|c|c|c|c|c|c|c|}
\hline \multirow{4}{*}{$\begin{array}{l}\begin{array}{c}\text { GAMA well } \\
\text { identification } \\
\text { number }\end{array} \\
\text { Benchmark type } \\
\text { Benchmark level } \\
\text { [RL] }\end{array}$} & \multicolumn{2}{|c|}{$\begin{array}{c}\text { Radon-222 } \\
\text { (pCi/L) } \\
(82303)\end{array}$} & \multicolumn{2}{|c|}{$\begin{array}{c}\text { Gross alpha radioactivity, } \\
\text { 72-hour count } \\
\text { (pCi/L) } \\
(62636)^{1}\end{array}$} & \multicolumn{2}{|c|}{$\begin{array}{c}\text { Gross alpha radioactivity, } \\
\text { 30-day count } \\
\text { (pCi/L) } \\
(62639)\end{array}$} & \multicolumn{2}{|c|}{$\begin{array}{c}\text { Gross beta radioactivity, } \\
\text { 72-hour count } \\
\text { (pCi/L) } \\
(62642)^{1}\end{array}$} & \multicolumn{2}{|c|}{$\begin{array}{c}\text { Gross beta radioactivity, } \\
\text { 30-day count } \\
\text { (pCi/L) } \\
(62645)\end{array}$} & \multicolumn{2}{|c|}{$\begin{array}{l}\text { Tritium } \\
\text { (pCi/L) } \\
(07000)\end{array}$} \\
\hline & \multicolumn{2}{|c|}{ Proposed MCL-US } & \multicolumn{2}{|c|}{ MCL-US ${ }^{2}$} & \multicolumn{2}{|c|}{ MCL-US ${ }^{2}$} & \multicolumn{2}{|c|}{ MCL-CA } & \multicolumn{2}{|c|}{ MCL-CA } & \multicolumn{2}{|c|}{ MCL-CA } \\
\hline & \multicolumn{2}{|l|}{4,000} & \multicolumn{2}{|l|}{15} & \multicolumn{2}{|l|}{15} & \multicolumn{2}{|l|}{50} & \multicolumn{2}{|l|}{50} & \multicolumn{2}{|l|}{20,000} \\
\hline & Result \pm CSU & $\operatorname{ssL}_{c}$ & Result \pm CSU & $s s_{c}$ & Result \pm CSU & $\operatorname{ssL}_{\mathrm{c}}$ & Result \pm CSU & ssL $_{c}$ & Result \pm CSU & $s s_{c}$ & Result \pm CSU & ssL $_{c}$ \\
\hline \multicolumn{13}{|c|}{ Madera/Chowchilla-Kings Shallow Aquifer study unit (73 to 77 wells sampled)_Continued } \\
\hline \multicolumn{13}{|c|}{ Kings study area ( 45 to 49 wells sampled) —Continued } \\
\hline S3-MACK-K11 & $1,010 \pm 58$ & 11 & ${ }^{1} 61.8 \pm 7.7$ & 2.0 & $64.3 \pm 8.4$ & 1.7 & ${ }^{1} 29.7 \pm 2.9$ & 1.6 & $41.8 \pm 3.8$ & 1.5 & $0.40 \pm 0.62$ & 0.39 \\
\hline S3-MACK-K12 & $585 \pm 36$ & 14 & $12.0 \pm 2.1$ & 1.2 & $9.0 \pm 1.9$ & 1.8 & $3.74 \pm 0.95$ & 1.5 & $9.9 \pm 1.3$ & 1.5 & $2.8 \pm 0.61$ & 0.23 \\
\hline S3-MACK-K13 & $597 \pm 37$ & 13 & $3.2 \pm 2.1$ & 3.2 & - \pm 2.1 & 3.9 & $3.2 \pm 1.1$ & 1.8 & $3.7 \pm 1.1$ & 1.7 & $9.7 \pm 0.41$ & 0.21 \\
\hline S3-MACK-K14 & $882 \pm 51$ & 13 & $2.77 \pm 0.95$ & 1.2 & - \pm 0.87 & 1.7 & $3.07 \pm 0.93$ & 1.5 & - \pm 0.81 & 1.5 & $12.7 \pm 0.41$ & 0.21 \\
\hline S3-MACK-K15 & $369 \pm 25$ & 13 & $15.9 \pm 2.2$ & 0.74 & $17.8 \pm 2.4$ & 0.74 & $1.97 \pm 0.77$ & 1.3 & $9.8 \pm 1.2$ & 1.3 & $10.5 \pm 0.41$ & 0.21 \\
\hline S3-MACK-K16 & $638 \pm 39$ & 13 & $94 \pm 13$ & 3.5 & $81 \pm 11$ & 3.9 & $15.0 \pm 2.6$ & 3.5 & $* 56.4 \pm 5.8$ & 3.6 & $9.0 \pm 0.61$ & 0.23 \\
\hline S3-MACK-K17 & $389 \pm 25$ & 11 & $3.55 \pm 0.74$ & 0.74 & $3.21 \pm 0.66$ & 0.60 & $1.81 \pm 0.78$ & 1.3 & $2.89 \pm 0.77$ & 1.2 & $10.8 \pm 0.45$ & 0.42 \\
\hline S3-MACK-K18 & $584 \pm 35$ & 11 & $154 \pm 18$ & 2.9 & $* 133 \pm 16$ & 3.7 & ${ }^{1} 43.0 \pm 4.2$ & 2.5 & $* 109.0 \pm 9.7$ & 1.9 & $6.0 \pm 0.62$ & 0.39 \\
\hline S3-MACK-K19 & $551 \pm 33$ & 11 & ${ }^{1} 87 \pm 12$ & 4.7 & $* 80 \pm 11$ & 3.5 & ${ }^{1} 27.5 \pm 3.1$ & 2.6 & $* 72.5 \pm 6.6$ & 2.4 & $13.3 \pm 0.49$ & 0.30 \\
\hline S3-MACK-K20 & $1,057 \pm 60$ & 11 & $79 \pm 10$ & 2.8 & $70.8 \pm 8.9$ & 2.3 & $14.1 \pm 2.1$ & 2.5 & $* 52.2 \pm 5.3$ & 2.6 & $16.3 \pm 0.49$ & 0.30 \\
\hline S3-MACK-K21 & $649 \pm 38$ & 10 & ${ }^{1} 35.8 \pm 4.7$ & 1.7 & $34.1 \pm 4.5$ & 1.5 & ${ }^{1} 10.1 \pm 1.3$ & 1.5 & $26.7 \pm 2.7$ & 1.3 & $13.6 \pm 0.49$ & 0.30 \\
\hline S3-MACK-K22 & $934 \pm 53$ & 11 & $8.9 \pm 1.5$ & 1.1 & $10.2 \pm 1.6$ & 1.0 & $11.5 \pm 1.4$ & 1.3 & $12.4 \pm 1.5$ & 1.4 & $9.0 \pm 0.46$ & 0.32 \\
\hline S3-MACK-K23 & $269 \pm 19$ & 11 & - \pm 0.43 & 0.76 & - \pm 0.44 & 0.71 & $20.6 \pm 2.1$ & 1.3 & $19.2 \pm 1.9$ & 1.1 & $6.2 \pm 0.46$ & 0.32 \\
\hline S3-MACK-K24 & $1,211 \pm 68$ & 12 & $47.8 \pm 6.1$ & 2.1 & $34.0 \pm 4.6$ & 1.7 & ${ }^{1} 11.9 \pm 1.4$ & 1.4 & $26.9 \pm 2.6$ & 1.5 & $23.4 \pm 0.46$ & 0.33 \\
\hline S3-MACK-K25 & $424 \pm 28$ & 13 & $9.9 \pm 1.6$ & 1.2 & $6.6 \pm 1.2$ & 0.9 & $4.81 \pm 0.82$ & 1.1 & $7.24 \pm 0.98$ & 1.0 & $14.0 \pm 0.62$ & 0.39 \\
\hline S3-MACK-K26 & $350 \pm 23$ & 11 & $5.44 \pm 0.93$ & 0.68 & $5.71 \pm 0.95$ & 0.65 & $2.48 \pm 0.77$ & 1.3 & $3.88 \pm 0.80$ & 1.2 & $10.5 \pm 0.62$ & 0.39 \\
\hline S3-MACK-K27 & $528 \pm 32$ & 12 & $46.7 \pm 6.7$ & 3.6 & $43.5 \pm 6.4$ & 3.7 & $9.1 \pm 2.0$ & 3.0 & $32.8 \pm 3.6$ & 3.0 & $8.3 \pm 0.45$ & 0.42 \\
\hline S3-MACK-K28 & $395 \pm 25$ & 12 & $8.0 \pm 1.5$ & 1.3 & $7.3 \pm 1.5$ & 1.4 & $1.43 \pm 0.69$ & 1.2 & $2.68 \pm 0.78$ & 1.2 & $7.4 \pm 0.61$ & 0.23 \\
\hline S3-MACK-K29 & $256 \pm 19$ & 13 & $11.1 \pm 2.5$ & 2.5 & $7.8 \pm 2.3$ & 2.7 & $7.8 \pm 1.7$ & 2.5 & $7.2 \pm 1.7$ & 2.6 & $9.5 \pm 0.61$ & 0.23 \\
\hline
\end{tabular}


Table 11. Results for analyses of radioactive constituents in groundwater samples collected for the Madera/Chowchilla-Kings Shallow Aquifer study unit, Groundwater Ambient Monitoring and Assessment (GAMA) Priority Basin Project, California, August 2013 to April 2014.-Continued

[The five-digit USGS parameter code below the constituent name is used to uniquely identify a specific constituent or property. Samples from all 77 grid wells were analyzed, unless noted as not collected (nc). Information about the constituents given in table $3 C$. Measured values less than the sample-specific critical level ( $\mathrm{ssL}_{\mathrm{C}}$ ) are reported as non-detections (-). GAMA well identification numbers: S3-MACK-K, Madera/Chowchilla-Kings Shallow Aquifer Kings study-area grid well; S3-MACK-M, Madera/Chowchilla-Kings Shallow Aquifer Madera/Chowchilla study-area grid well. Benchmark type and benchmark level as of April 30, 2014. Benchmark types: Maximum contaminant level benchmarks are listed as MCL-US when the MCL-US and MCL-CA are identical, and as MCL-CA when the MCL-CA is lower than the MCL-US or no MCL-US exists. MCL-CA, State of California maximum contaminant level; MCL-US, U.S. Environmental Protection Agency maximum contaminant level. Abbreviations: CSU, 1-sigma combined standard uncertainty; pCi/L, picocuries per liter; RL, reporting level; USGS, U.S. Geological Survey; —, not detected; \pm , plus or minus; *, activity greater than the benchmark level]

\begin{tabular}{|c|c|c|c|c|c|c|c|c|c|c|c|c|}
\hline \multirow{4}{*}{$\begin{array}{l}\begin{array}{c}\text { GAMA well } \\
\text { identification } \\
\text { number }\end{array} \\
\text { Benchmark type } \\
\text { Benchmark level } \\
\text { [RL] }\end{array}$} & \multicolumn{2}{|c|}{$\begin{array}{c}\text { Radon-222 } \\
(\mathrm{pCi} / \mathrm{L}) \\
(82303)\end{array}$} & \multicolumn{2}{|c|}{$\begin{array}{c}\text { Gross alpha radioactivity, } \\
\text { 72-hour count } \\
\text { (pCi/L) } \\
(62636)^{1}\end{array}$} & \multicolumn{2}{|c|}{$\begin{array}{c}\text { Gross alpha radioactivity, } \\
\text { 30-day count } \\
\text { (pCi/L) } \\
(62639)\end{array}$} & \multicolumn{2}{|c|}{$\begin{array}{c}\text { Gross beta radioactivity, } \\
\text { 72-hour count } \\
\text { (pCi/L) } \\
(62642)^{1}\end{array}$} & \multicolumn{2}{|c|}{$\begin{array}{c}\text { Gross beta radioactivity, } \\
\text { 30-day count } \\
\text { (pCi/L) } \\
(62645)\end{array}$} & \multicolumn{2}{|c|}{$\begin{array}{l}\text { Tritium } \\
\text { (pCi/L) } \\
\text { (07000) }\end{array}$} \\
\hline & \multicolumn{2}{|c|}{ Proposed MCL-US } & \multicolumn{2}{|c|}{ MCL-US ${ }^{2}$} & \multicolumn{2}{|c|}{ MCL-US ${ }^{2}$} & \multicolumn{2}{|c|}{ MCL-CA } & \multicolumn{2}{|c|}{ MCL-CA } & \multicolumn{2}{|c|}{ MCL-CA } \\
\hline & \multicolumn{2}{|l|}{4,000} & \multicolumn{2}{|l|}{15} & \multicolumn{2}{|l|}{15} & \multicolumn{2}{|l|}{50} & \multicolumn{2}{|l|}{50} & \multicolumn{2}{|l|}{20,000} \\
\hline & Result \pm CSU & ssL $_{c}$ & Result \pm CSU & $\mathbf{s s L}_{\mathrm{c}}$ & Result \pm CSU & ssL $_{c}$ & Result \pm CSU & ssL $_{c}$ & Result \pm CSU & $\mathbf{s s L}_{\mathrm{c}}$ & Result \pm CSU & ssL $_{\mathrm{c}}$ \\
\hline \multicolumn{13}{|c|}{ Madera/Chowchilla-Kings Shallow Aquifer study unit (73 to 77 wells sampled)_Continued } \\
\hline \multicolumn{13}{|c|}{ Kings study area ( 45 to 49 wells sampled)_-Continued } \\
\hline S3-MACK-K30 & $317 \pm 21$ & 11 & $1.44 \pm 0.54$ & 0.73 & $1.66 \pm 0.47$ & 0.50 & $3.31 \pm 0.80$ & 1.2 & $4.08 \pm 0.82$ & 1.2 & $11.2 \pm 0.45$ & 0.42 \\
\hline S3-MACK-K31 & $272 \pm 19$ & 11 & $1.03 \pm 0.44$ & 0.63 & - \pm 0.28 & 0.42 & $5.54 \pm 0.91$ & 1.2 & $2.94 \pm 0.69$ & 1.0 & - \pm 0.62 & 0.39 \\
\hline S3-MACK-K32 & $601 \pm 36$ & 11 & $1.46 \pm 0.43$ & 0.53 & $1.74 \pm 0.43$ & 0.48 & $3.80 \pm 0.72$ & 1.0 & $2.75 \pm 0.67$ & 1.0 & $10.0 \pm 0.62$ & 0.39 \\
\hline S3-MACK-K33 & $273 \pm 19$ & 12 & $8.7 \pm 1.6$ & 1.1 & $5.3 \pm 1.3$ & 1.6 & $5.2 \pm 1.1$ & 1.6 & $5.19 \pm 0.93$ & 1.3 & $6.5 \pm 0.49$ & 0.30 \\
\hline S3-MACK-K34 & $393 \pm 25$ & 12 & $5.6 \pm 1.2$ & 1.2 & $8.1 \pm 1.5$ & 1.3 & $16.8 \pm 2.1$ & 2.1 & $8.0 \pm 1.2$ & 1.5 & $4.2 \pm 0.49$ & 0.30 \\
\hline S3-MACK-K35 & $506 \pm 31$ & 11 & $1.31 \pm 0.50$ & 0.66 & - \pm 0.50 & 0.83 & $4.47 \pm 0.87$ & 1.2 & $1.78 \pm 0.81$ & 1.4 & $4.8 \pm 0.46$ & 0.32 \\
\hline S3-MACK-K36 & $1,498 \pm 83$ & 11 & $3.67 \pm 0.88$ & 0.93 & $2.01 \pm 0.82$ & 1.2 & $4.20 \pm 0.87$ & 1.3 & $5.40 \pm 0.97$ & 1.3 & $12.2 \pm 0.39$ & 0.31 \\
\hline S3-MACK-K37 & $312 \pm 21$ & 11 & - \pm 0.47 & 0.79 & - \pm 0.42 & 0.72 & $3.34 \pm 0.83$ & 1.3 & $2.88 \pm 0.80$ & 1.3 & $11.2 \pm 0.46$ & 0.32 \\
\hline S3-MACK-K38 & $280 \pm 20$ & 13 & $3.7 \pm 1.4$ & 1.9 & - \pm 1.2 & 1.8 & $6.7 \pm 1.2$ & 1.5 & $6.7 \pm 1.0$ & 1.2 & $12.7 \pm 0.49$ & 0.30 \\
\hline S3-MACK-K39 & $635 \pm 39$ & 14 & $16.6 \pm 2.7$ & 2.0 & $16.0 \pm 2.6$ & 1.9 & $10.6 \pm 1.6$ & 2.0 & $10.4 \pm 1.6$ & 2.1 & $8.2 \pm 0.62$ & 0.39 \\
\hline S3-MACK-K40 & $635 \pm 37$ & 11 & $28.8 \pm 4.8$ & 3.3 & $35.3 \pm 5.2$ & 2.5 & $15.2 \pm 2.2$ & 2.7 & $19.4 \pm 2.5$ & 2.7 & $9.7 \pm 0.62$ & 0.39 \\
\hline S3-MACK-K41 & $654 \pm 39$ & 12 & $7.6 \pm 1.9$ & 2.1 & $11.0 \pm 2.3$ & 2.1 & $7.1 \pm 1.6$ & 2.3 & $18.9 \pm 2.3$ & 2.2 & $9.2 \pm 0.45$ & 0.42 \\
\hline S3-MACK-K42 & $314 \pm 22$ & 13 & $8.7 \pm 2.2$ & 2.4 & $7.7 \pm 2.1$ & 2.4 & $5.8 \pm 1.6$ & 2.6 & $6.3 \pm 1.6$ & 2.5 & $8.1 \pm 0.41$ & 0.21 \\
\hline S3-MACK-K43 & $305 \pm 20$ & 11 & $6.0 \pm 1.9$ & 2.3 & $6.0 \pm 2.0$ & 2.6 & $6.6 \pm 1.8$ & 2.7 & $8.2 \pm 1.9$ & 2.8 & $11.3 \pm 0.62$ & 0.39 \\
\hline S3-MACK-K44 & $585 \pm 35$ & 11 & $7.6 \pm 2.5$ & 3.2 & $6.5 \pm 2.0$ & 2.5 & $7.8 \pm 1.9$ & 2.8 & $15.1 \pm 2.3$ & 2.8 & $11.6 \pm 0.54$ & 0.27 \\
\hline S3-MACK-K45 & $171 \pm 14$ & 11 & $3.1 \pm 1.4$ & 2.1 & $3.5 \pm 1.2$ & 1.5 & $6.1 \pm 1.1$ & 1.6 & $7.8 \pm 1.2$ & 1.5 & $6.4 \pm 0.46$ & 0.33 \\
\hline S3-MACK-K46 & $3,920 \pm 210$ & 11 & $6.1 \pm 1.2$ & 1.0 & $3.85 \pm 0.92$ & 1.0 & $3.95 \pm 0.84$ & 1.2 & $6.4 \pm 1.0$ & 1.3 & $8.6 \pm 0.49$ & 0.30 \\
\hline S3-MACK-K47 & $114 \pm 13$ & 14 & - \pm 1.8 & 2.8 & $4.6 \pm 1.9$ & 2.6 & $5.4 \pm 1.9$ & 3.1 & $7.2 \pm 2.0$ & 3.1 & $6.5 \pm 0.46$ & 0.32 \\
\hline S3-MACK-K48 & $322 \pm 22$ & 11 & $3.5 \pm 1.2$ & 1.5 & $2.29 \pm 0.99$ & 1.4 & $3.2 \pm 1.1$ & 1.7 & $5.1 \pm 1.1$ & 1.7 & $4.0 \pm 0.39$ & 0.31 \\
\hline
\end{tabular}


Table 11. Results for analyses of radioactive constituents in groundwater samples collected for the Madera/Chowchilla-Kings Shallow Aquifer study unit, Groundwater Ambient Monitoring and Assessment (GAMA) Priority Basin Project, California, August 2013 to April 2014.-Continued

[The five-digit USGS parameter code below the constituent name is used to uniquely identify a specific constituent or property. Samples from all 77 grid wells were analyzed, unless noted as not collected (nc). Information about the constituents given in table 3C. Measured values less than the sample-specific critical level (ssL $\mathrm{C}_{\mathrm{C}}$ ) are reported as non-detections (-). GAMA well identification numbers: S3-MACK-K, Madera/Chowchilla-Kings Shallow Aquifer Kings study-area grid well; S3-MACK-M, Madera/Chowchilla-Kings Shallow Aquifer Madera/Chowchilla study-area grid well. Benchmark type and benchmark level as of April 30, 2014. Benchmark types: Maximum contaminant level benchmarks are listed as MCL-US when the MCL-US and MCL-CA are identical, and as MCL-CA when the MCL-CA is lower than the MCL-US or no MCL-US exists. MCL-CA, State of California maximum contaminant level; MCL-US, U.S. Environmental Protection Agency maximum contaminant level. Abbreviations: CSU, 1-sigma combined standard uncertainty; pCi/L, picocuries per liter; RL, reporting level; USGS, U.S. Geological Survey; —, not detected; \pm , plus or minus; *, activity greater than the benchmark level]

\begin{tabular}{|c|c|c|c|c|c|c|}
\hline $\begin{array}{l}\text { GAMA well } \\
\text { identification } \\
\text { number }\end{array}$ & $\begin{array}{l}\text { Radon-222 } \\
\text { (pCi/L) } \\
(82303)\end{array}$ & $\begin{array}{c}\text { Gross alpha radioactivity, } \\
\text { 72-hour count } \\
\text { (pCi/L) } \\
(62636)^{1}\end{array}$ & $\begin{array}{c}\text { Gross alpha radioactivity, } \\
\text { 30-day count } \\
\text { (pCi/L) } \\
\text { (62639) }\end{array}$ & $\begin{array}{c}\text { Gross beta radioactivity, } \\
\text { 72-hour count } \\
\text { (pCi/L) } \\
(62642)^{1}\end{array}$ & $\begin{array}{c}\text { Gross beta radioactivity, } \\
\text { 30-day count } \\
\text { (pCi/L) } \\
(62645)\end{array}$ & $\begin{array}{l}\text { Tritium } \\
\text { (pCi/L) } \\
(07000)\end{array}$ \\
\hline Benchmark type & Proposed MCL-US & MCL-US ${ }^{2}$ & MCL-US ${ }^{2}$ & MCL-CA & MCL-CA & MCL-CA \\
\hline Benchmark level & 4,000 & 15 & 15 & 50 & 50 & 20,000 \\
\hline [RL] & Result \pm CSU & Result \pm CSU & Result \pm CSU & Result \pm CSU & Result \pm CSU & Result \pm CSU \\
\hline
\end{tabular}

Madera/Chowchilla-Kings Shallow Aquifer study unit (73 to 77 wells sampled)—Continued

Kings study area ( 45 to 49 wells sampled)-Continued

\begin{tabular}{|c|c|c|c|c|c|c|c|c|c|c|c|c|}
\hline S3-MACK-K49 & $165 \pm 15$ & 13 & $12.7 \pm 2.6$ & 2.6 & $5.3 \pm 1.8$ & 2.5 & $10.4 \pm 1.9$ & 2.6 & $10.2 \pm 1.9$ & 2.6 & $7.7 \pm 0.51$ & 0.26 \\
\hline
\end{tabular}

${ }^{1}$ The 72-hour holding time was exceeded by 1-4 days. Note: the S3-MACK-K21 replicate pair late sample count for the 62636 and 62642 analyses were within acceptable ranges.

${ }^{2}$ The MCL-US benchmark for gross alpha activity applies to adjusted gross alpha, which is equal to measured gross alpha activity minus uranium activity. Unadjusted gross alpha activity results from the laboratory are reported, but only those with adjusted gross alpha values greater than the MCL-US benchmark are noted with an asterisk. 
Table 12. Results for analyses of isotopic tracers in groundwater samples collected for the Madera/Chowchilla-Kings Shallow Aquifer study unit, Groundwater Ambient Monitoring and Assessment (GAMA) Priority Basin Project, California, August 2013 to April 2014.

[The five-digit USGS parameter code below the constituent name is used to uniquely identify a specific constituent or property. Information about the constituents given in table $3 C$. Samples from all 77 grid wells were analyzed, unless noted as not collected (nc). Stable isotope ratios of carbon (C), hydrogen $(\mathrm{H})$, oxygen $(\mathrm{O})$, and nitrogen $(\mathrm{N})$ are reported in the standard delta notation $(\delta)$, the ratio of a heavier isotope to more common lighter isotope of that element, relative to a standard reference material. GAMA well identification numbers: S3-MACK-K, Madera/Chowchilla-Kings Shallow Aquifer Kings study-area grid well; S3-MACK-M, Madera/Chowchilla-Kings Shallow Aquifer Madera/Chowchilla study-area grid well. Benchmark type and benchmark level as of April 30, 2014. Abbreviations: DIC, dissolved inorganic carbon; na, not available; USGS, U.S. Geological Survey]

\begin{tabular}{|c|c|c|c|c|c|c|}
\hline $\begin{array}{c}\text { GAMA well } \\
\text { identification } \\
\text { number }\end{array}$ & $\begin{array}{c}\delta^{13} \mathrm{C} \text { in DIC } \\
\text { (per mil) } \\
(82081)\end{array}$ & $\begin{array}{c}\text { Carbon-14 in DIC } \\
\text { (percent modern) } \\
(49933)\end{array}$ & $\begin{array}{c}\delta^{2} \mathrm{H} \text { in water } \\
\text { (per mil) } \\
(82082)\end{array}$ & $\begin{array}{c}\delta^{18} 0 \text { in water } \\
\text { (per mil) } \\
(82085)\end{array}$ & $\begin{array}{c}\delta^{15} \mathrm{~N} \text { of nitrate } \\
\text { (per mil) } \\
(82690)\end{array}$ & $\begin{array}{c}\delta^{18} 0 \text { of nitrate } \\
\text { (per mil) } \\
(63041)\end{array}$ \\
\hline Benchmark type & na & na & na & na & na & na \\
\hline Benchmark level & na & na & na & na & na & na \\
\hline Reporting level & na & na & na & na & na & na \\
\hline S3-MACK-M01 & -16.14 & 71.15 & -99.5 & -13.44 & $\mathrm{nc}$ & nc \\
\hline S3-MACK-M02 & -14.44 & 73.49 & -70.7 & -9.79 & 4.26 & -2.53 \\
\hline S3-MACK-M03 & -8.90 & 108.4 & -59.8 & -7.78 & 12.05 & 1.43 \\
\hline S3-MACK-M04 & -14.68 & 103.3 & -66.2 & -8.81 & 4.37 & 0.08 \\
\hline S3-MACK-M08 & -13.18 & 109.1 & -79.4 & -10.83 & 5.57 & -0.26 \\
\hline S3-MACK-M09 & -15.04 & 102.7 & -69.5 & -9.23 & 4.60 & 0.86 \\
\hline S3-MACK-M10 & -12.55 & 112.0 & -70.7 & -9.66 & 10.80 & 1.91 \\
\hline S3-MACK-M11 & -14.72 & 97.05 & -79.2 & -10.87 & 4.50 & -1.24 \\
\hline S3-MACK-M12 & -16.07 & 85.30 & -86.8 & -11.60 & 5.05 & 0.31 \\
\hline S3-MACK-M13 & -14.61 & 99.57 & -75.8 & -10.39 & 4.33 & -0.72 \\
\hline S3-MACK-M14 & -14.09 & 110.5 & -85.2 & -11.65 & 3.47 & 0.64 \\
\hline S3-MACK-M15 & -15.56 & 87.19 & -71.8 & -9.69 & 5.58 & -0.81 \\
\hline S3-MACK-M21 & -12.95 & 103.6 & -67.8 & -9.20 & 7.64 & 2.63 \\
\hline S3-MACK-M22 & -18.51 & 70.49 & -63.2 & -8.09 & 3.76 & 0.99 \\
\hline S3-MACK-M23 & -16.47 & 105.8 & -94.3 & -12.85 & $\mathrm{nc}$ & nc \\
\hline S3-MACK-M24 & -17.91 & 76.93 & -57.8 & -7.69 & 3.40 & 1.88 \\
\hline S3-MACK-M25 & -13.82 & 81.55 & -57.6 & -7.38 & 4.18 & 0.69 \\
\hline S3-MACK-M26 & -13.13 & 80.87 & -57.1 & -7.51 & 4.06 & 1.20 \\
\hline S3-MACK-M27 & -15.56 & 92.43 & -72.3 & -9.85 & 4.46 & 0.02 \\
\hline S3-MACK-M28 & -13.38 & 10.77 & -58.7 & -7.67 & nc & nc \\
\hline
\end{tabular}


Table 12. Results for analyses of isotopic tracers in groundwater samples collected for the Madera/Chowchilla-Kings Shallow Aquifer study unit, Groundwater Ambient Monitoring and Assessment (GAMA) Priority Basin Project, California, August 2013 to April 2014.— Continued

[The five-digit USGS parameter code below the constituent name is used to uniquely identify a specific constituent or property. Information about the constituents given in table 3C. Samples from all 77 grid wells were analyzed, unless noted as not collected (nc). Stable isotope ratios of carbon (C), hydrogen $(\mathrm{H})$, oxygen $(\mathrm{O})$, and nitrogen $(\mathrm{N})$ are reported in the standard delta notation $(\delta)$, the ratio of a heavier isotope to more common lighter isotope of that element, relative to a standard reference material. GAMA well identification numbers: S3-MACK-K, Madera/Chowchilla-Kings Shallow Aquifer Kings study-area grid well; S3-MACK-M, Madera/Chowchilla-Kings Shallow Aquifer Madera/Chowchilla study-area grid well. Benchmark type and benchmark level as of April 30, 2014. Abbreviations: DIC, dissolved inorganic carbon; na, not available; USGS, U.S. Geological Survey]

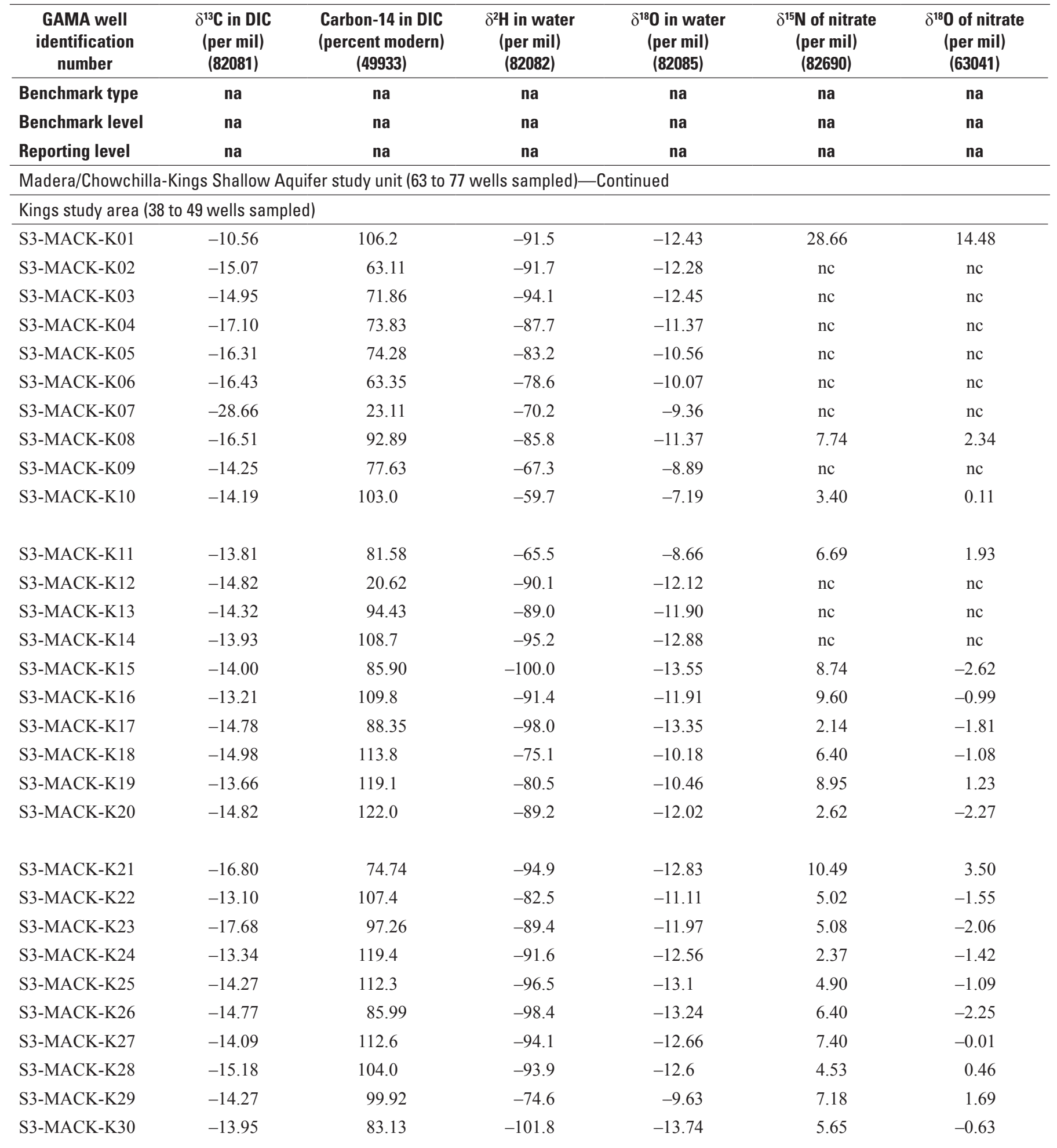


Table 12. Results for analyses of isotopic tracers in groundwater samples collected for the Madera/Chowchilla-Kings Shallow Aquifer study unit, Groundwater Ambient Monitoring and Assessment (GAMA) Priority Basin Project, California, August 2013 to April 2014.Continued

[The five-digit USGS parameter code below the constituent name is used to uniquely identify a specific constituent or property. Information about the constituents given in table 3C. Samples from all 77 grid wells were analyzed, unless noted as not collected (nc). Stable isotope ratios of carbon (C), hydrogen $(\mathrm{H})$, oxygen $(\mathrm{O})$, and nitrogen $(\mathrm{N})$ are reported in the standard delta notation $(\delta)$, the ratio of a heavier isotope to more common lighter isotope of that element, relative to a standard reference material. GAMA well identification numbers: S3-MACK-K, Madera/Chowchilla-Kings Shallow Aquifer Kings study-area grid well; S3-MACK-M, Madera/Chowchilla-Kings Shallow Aquifer Madera/Chowchilla study-area grid well. Benchmark type and benchmark level as of April 30, 2014. Abbreviations: DIC, dissolved inorganic carbon; na, not available; USGS, U.S. Geological Survey]

\begin{tabular}{|c|c|c|c|c|c|c|}
\hline $\begin{array}{l}\text { GAMA well } \\
\text { identification } \\
\text { number }\end{array}$ & $\begin{array}{c}\delta^{13} \mathrm{C} \text { in DIC } \\
\text { (per mil) } \\
\text { (82081) }\end{array}$ & $\begin{array}{c}\text { Carbon-14 in DIC } \\
\text { (percent modern) } \\
\text { (49933) }\end{array}$ & $\begin{array}{c}\delta^{2} \mathrm{H} \text { in water } \\
\text { (per mil) } \\
(\mathbf{8 2 0 8 2 )}\end{array}$ & $\begin{array}{c}\delta^{18} 0 \text { in water } \\
\text { (per mil) } \\
\text { (82085) }\end{array}$ & $\begin{array}{c}\delta^{15} \mathrm{~N} \text { of nitrate } \\
\text { (per mil) } \\
\text { (82690) }\end{array}$ & $\begin{array}{c}\delta^{18} 0 \text { of nitrate } \\
\text { (per mil) } \\
(63041)\end{array}$ \\
\hline Benchmark type & na & na & na & na & na & na \\
\hline Benchmark level & na & na & na & na & na & na \\
\hline Reporting level & na & na & na & na & na & na \\
\hline \multicolumn{7}{|c|}{ Madera/Chowchilla-Kings Shallow Aquifer study unit (63 to 77 wells sampled)—Continued } \\
\hline \multicolumn{7}{|c|}{ Kings study area (38 to 49 wells) -Continued } \\
\hline S3-MACK-K31 & -15.44 & 81.99 & -96.2 & -13.08 & 3.60 & -1.72 \\
\hline S3-MACK-K32 & -15.96 & 103.0 & -99.5 & -13.53 & 4.05 & -0.92 \\
\hline S3-MACK-K33 & -16.33 & 112.0 & -79.6 & -10.31 & 3.39 & 2.26 \\
\hline S3-MACK-K34 & -14.37 & 107.5 & -68.6 & -9.18 & 6.57 & 0.42 \\
\hline S3-MACK-K35 & -16.29 & 106.4 & -90.0 & -12.06 & 6.63 & -1.46 \\
\hline S3-MACK-K36 & -16.23 & 110.9 & -77.1 & -10.32 & 3.43 & 1.06 \\
\hline S3-MACK-K37 & -19.01 & 91.37 & -77.4 & -9.76 & 8.65 & -4.00 \\
\hline S3-MACK-K38 & -16.30 & 113.9 & -86.6 & -11.56 & 4.21 & 2.60 \\
\hline S3-MACK-K39 & -15.58 & 111.7 & -86.9 & -11.52 & 4.14 & 2.39 \\
\hline S3-MACK-K40 & -15.13 & 109.2 & -90.7 & -12.18 & 6.36 & 2.05 \\
\hline S3-MACK-K41 & -14.62 & 115.2 & -74.1 & -9.82 & 6.32 & 2.24 \\
\hline S3-MACK-K42 & -14.63 & 90.89 & -63.0 & -8.36 & 4.47 & 2.90 \\
\hline S3-MACK-K43 & -15.92 & 113.0 & -77.3 & -9.91 & 5.38 & 4.16 \\
\hline S3-MACK-K44 & -16.02 & 110.6 & -72.1 & -9.26 & 6.92 & 7.32 \\
\hline S3-MACK-K45 & -16.39 & 102.5 & -89.3 & -11.60 & 5.26 & 8.87 \\
\hline S3-MACK-K46 & -13.25 & 114.6 & -96.1 & -12.98 & $\mathrm{nc}$ & $\mathrm{nc}$ \\
\hline S3-MACK-K47 & -16.22 & 107.4 & -57.7 & -7.62 & 5.84 & 2.38 \\
\hline S3-MACK-K48 & -16.44 & 104.8 & -63.4 & -7.99 & 5.68 & 3.38 \\
\hline S3-MACK-K49 & -16.22 & 104.2 & -52.5 & -6.47 & 3.82 & 4.04 \\
\hline
\end{tabular}


Table 13. Microbial indicators detected in groundwater samples collected for the Madera/Chowchilla-Kings Shallow Aquifer study unit, Groundwater Ambient Monitoring and Assessment (GAMA) study, California, August 2013 to April 2014.

[The five digit number in parentheses below the constituent name is the U.S. Geological Survey parameter code used to uniquely identify a specific constituent or property. Samples from 73 grid wells were analyzed for E. coli and enterococci, and samples from 72 grid wells were analyzed for total coliform, but only samples with a detection are listed. Information about the analytes given in table $3 D$. GAMA well identification numbers: S3-MACK-K, Madera/Chowchilla-Kings Shallow Aquifer Kings study-area grid well; S3-MACK-M, Madera/Chowchilla-Kings Shallow Aquifer Madera/ Chowchilla study-area grid well. Benchmark type and benchmark level as of April 30, 2014. Benchmark types: TT-US, U.S. Environmental Protection Agency (EPA) treatment technique — a required process intended to reduce the level of contamination in drinking water; MCL-US, EPA maximum contaminant level. Abbreviations: M, presence verified but not quantified; na, not available; - , not detected]

\begin{tabular}{|c|c|c|c|}
\hline $\begin{array}{c}\text { GAMA well } \\
\text { identification } \\
\text { number }\end{array}$ & $\begin{array}{c}\text { Escherichia } \\
\text { coli } \\
\text { (E. coli) } \\
\text { (99596) }\end{array}$ & $\begin{array}{l}\text { Total coliform } \\
\text { (including } \\
\text { fecal coliform } \\
\text { and } E \text {. coli) } \\
\text { (99595) }\end{array}$ & $\begin{array}{c}\text { Enterococci } \\
(31719)\end{array}$ \\
\hline Benchmark type & TT-US & MCL-US & TT-US \\
\hline $\begin{array}{l}\text { Benchmark } \\
\text { level }\end{array}$ & $\begin{array}{l}\text { No fecal } \\
\text { coliforms are } \\
\text { allowed }\end{array}$ & $\begin{array}{l}5 \text { percent } \\
\text { of samples } \\
\text { per month }\end{array}$ & $\begin{array}{c}\text { No fecal } \\
\text { bacteria are } \\
\text { allowed }\end{array}$ \\
\hline \multicolumn{4}{|c|}{$\begin{array}{l}\text { Madera/Chowchilla-Kings Shallow Aquifer study area } \\
\text { ( } 72 \text { to } 73 \text { wells sampled) }\end{array}$} \\
\hline \multicolumn{4}{|c|}{ Madera/Chowchilla study area ( 25 to 27 wells sampled) ${ }^{1}$} \\
\hline S3-MACK-M20 & ${ }^{1}$ na & ${ }^{1}$ na & M \\
\hline S3-MACK-M21 & - & M & - \\
\hline \multicolumn{4}{|c|}{ Kings study area (46 to 47 wells sampled) ${ }^{1}$} \\
\hline S3-MACK-K01 & - & M & - \\
\hline S3-MACK-K06 & - & - & M \\
\hline S3-MACK-K10 & - & M & M \\
\hline S3-MACK-K11 & - & M & - \\
\hline S3-MACK-K18 & - & - & M \\
\hline S3-MACK-K20 & - & M & - \\
\hline S3-MACK-K33 & - & M & M \\
\hline S3-MACK-K34 & - & M & - \\
\hline S3-MACK-K43 & - & M & - \\
\hline S3-MACK-K46 & - & M & - \\
\hline S3-MACK-K47 & - & M & - \\
\hline
\end{tabular}

${ }^{1}$ Some samples were not analyzed because the incubator temperature sensors fluctuated outside of the required temperature range during the incubation. Four samples were not analyzed for E. coli (S3-MACK-M10, -M20, -K24, -K30), five samples were not analyzed for total coliform (S3-MACK-M04, -M10, -M20, -K24, -K30), and four samples were not analyzed for enterococci (S3-MACK-M06, -K03, -K24, -K30). 


\section{Appendix A}

This appendix includes descriptions and discussions of the methods used to collect and analyze groundwater samples and report the resulting water-quality data. These methods were selected to obtain representative samples of the groundwater from each well and to minimize the potential for contamination of the samples or other bias in the data. Procedures used to collect and assess quality-control (QC) data and the results of the QC assessments also are discussed.

In the Madera/Chowchilla-Kings Shallow Aquifer (MACK) study unit, groundwater samples were collected, and quality assurance (QA) procedures followed standard and modified U.S. Geological Survey (USGS) protocols from the National Water Quality Assessment Program (NAWQA; Koterba and others, 1995), the National Field Manual (NFM; U.S. Geological Survey, variously dated), and protocols described by Shelton and others (2001) and Wright and others (2005). The primary difference between procedures used in this study and those used by the NAWQA Program is that portable sampling chambers were not used in this study. Comparison of results for blanks collected by the Groundwater Ambient Monitoring and Assessment Program (GAMA) and blanks collected by NAWQA indicated that GAMA field-sampling protocols resulted in fewer instances of contamination than NAWQA field sampling protocols (Fram and others, 2012); thus, the lack of portable sampling chambers did not adversely affect the quality of the samples collected. The QA plan followed by the National Water Quality Laboratory (NWQL), the primary laboratory used to analyze samples for this study, is described in Maloney (2005) and Pirkey and Glodt (1998).

\section{Water Levels and Well Information}

Depth to water level was measured according to the "Groundwater Technical Procedures" of the U.S. Geological Survey (Cunningham and Schalk, 2011) prior to sample collection, when access was available. Water levels were measured by using steel tapes or electrical tapes, and all tapes were calibrated annually.

Well locations were verified by using a global positioning system (GPS), 1:24,000-scale USGS topographic maps, comparison with existing well information in USGS and state databases, and information provided by well owners, well-completion reports (WCRs), or other sources of construction information. Well locations and information were recorded by hand on field sheets. All information was verified and then uploaded into the USGS National Water Information System (NWIS; Website accessed January 28, 2015, at http://waterdata.usgs.gov/ca/nwis/). Well-owner and well-use information is confidential.

\section{Sample Collection and Analysis}

Samples were collected from 71 production wells and 6 short-screened monitoring wells open at depth intervals similar to the depth ranges of domestic wells (table 1). Production wells have pumps that bring the groundwater from the aquifer to a distribution system. A portable pump was used to collect samples from the monitoring wells because the monitoring wells did not have dedicated pumps.

In most cases, wells were pumped continuously to purge at least three casing volumes of water from the well prior to sampling (Wilde and others, 1999). During the period of sampling for the MACK study unit (August 2013 through April 2014), severe drought conditions limited the amount of groundwater that could be pumped from some of the wells. In some cases, continuous pumping was limited to 2 hours because of drawdown of the water table. A minimum of onecasing volume of groundwater was pumped from each well before sampling. The limits on pumping did not allow time to complete the sampling of a few wells; consequently, some constituent groups were not determined for these wells.

Production wells were sampled by using Teflon ${ }^{\circledR}$ tubing with brass and stainless-steel fittings attached to a sampling point (usually a hose-bib fitting) on the well-discharge pipe as close to the well head as possible. The sampling point was upstream from water-storage tanks and from well-head treatment system (if a system existed). If a chlorinating system was attached to the well, the chlorinator was shut off, when possible, before the well was purged and sampled, to clear all the chlorine out of the system. The absence of free chlorine was verified by using a $\mathrm{Hach}^{\circledR}$ field test kit.

Monitoring wells were sampled by using a portable Grundfos ${ }^{\circledR}$ submersible sampling pump with Teflon ${ }^{\circledR}$ tubing and stainless steel fittings. All samples were collected outdoors. Production-well samples were collected by connecting a 1- to 3-foot (ft) length of Teflon ${ }^{\circledR}$ tubing to the sampling point (Lane and others, 2003). Monitoring-well samples were collected by using a 3- to 10 -ft length of Teflon ${ }^{\circledR}$ tubing connected to the sampling point. All fittings and lengths of tubing were cleaned between samples (Wilde, 2004).

For the field measurements, groundwater was pumped through a flow-through chamber that was attached to the sampling point and fitted with a multi-probe meter that simultaneously measured the field water-quality indicators dissolved oxygen, temperature, $\mathrm{pH}$, and specific conductance. Field measurements were taken according to protocols in the NFM (Radtke and others, 2005; Wilde and Radtke, 2005; Lewis, 2006; Wilde, 2006; Wilde and others, 2006). All sensors on the multi-probe meter were calibrated daily. Measured dissolved-oxygen, temperature, $\mathrm{pH}$, and specificconductance values were recorded at 3- to 5-minute intervals, and when these values remained stable, samples for laboratory analyses were collected. 
Field measurements and instrument calibrations were recorded by hand on field-record sheets and electronically in the "Personal Computer Field Form" (PCFF) program. Analytical service requests to NWQL also were managed by PCFF, whereas analytical service requests for non-NWQL analysis were entered in laboratory-specific spreadsheets. Information from the PCFF was uploaded directly to the USGS NWIS at the end of each week of sample collection.

Prior to sample collection, polyethylene sample bottles were pre-rinsed three times with deionized water and then once with native sample water before sample collection. Samples requiring acidification were acidified to a $\mathrm{pH}$ between 1 and 2 with the appropriate acids by using ampoules of certified, traceable, concentrated acids obtained from the NWQL.

Temperature-sensitive samples were stored on ice prior to and during shipping to the various laboratories. The non-temperature-sensitive samples for stable isotopes of hydrogen and oxygen in water, tritium, and dissolved noble gases were shipped monthly. Temperature-sensitive or timesensitive samples for volatile organic compound s (VOCs), pesticides and pesticide degradates, trace elements, nutrients, major and minor ions, silica, total dissolved solids (TDS), laboratory alkalinity, perchlorate, radon-222, and gross alpha and gross beta radioactivity were shipped daily, whenever possible; samples for dissolved standard gases were shipped weekly. Samples for stable isotopes of nitrogen and oxygen in dissolved nitrate and stable isotopes of carbon in dissolved inorganic carbon and carbon-14 abundance were stored on ice, archived in a laboratory refrigerator or freezer, and only shipped after results for nitrate and laboratory alkalinity were received because alkalinity was used to determine the volumes required for processing.

Detailed sampling protocols for individual analyses and groups of analytes are described in Koterba and others (1995), the NFM (Wilde and others, 1999, 2004), and in the references for analytical methods listed in appendix table A-1; only brief descriptions are given here. Samples for analyses of VOCs were collected in 40-milliliter $(\mathrm{mL})$ sample vials that were purged with three vial volumes of unfiltered groundwater before bottom filling to eliminate atmospheric contamination. One-to-one hydrochloric acid to water $\left(\mathrm{HCl} / \mathrm{H}_{2} \mathrm{O}\right)$ solution was added as a preservative. Each sample analyzed for perchlorate was collected in a $125-\mathrm{mL}$ polystyrene bottle and then filtered in two or three $20-\mathrm{mL}$ aliquots of groundwater through a 0.20 -micrometer $(\mu \mathrm{m})$ pore-size Corning ${ }^{\mathbb{}}$ syringe-tip disk filter into a sterilized, $125-\mathrm{mL}$ polystyrene bottle. Samples for analysis of tritium were collected by bottom filling one 1-liter (L) polyethylene bottle or one 1-L glass bottle with unfiltered groundwater after, first, overfilling the bottles with one to three volumes of unfiltered groundwater. Samples for analysis of stable isotopes of hydrogen and oxygen in water were collected in a $60-\mathrm{mL}$ clear-glass bottle filled with unfiltered groundwater, sealed with a conical cap, and secured with electrical tape to prevent leakage and evaporation.

Samples for analysis of pesticides and pesticide degradates were collected in 20- to $40-\mathrm{mL}$ amber-glass vials. These samples were filtered through a 47-millimeter (mm) glass-fiber filter during collection.

Groundwater samples for trace elements, major and minor ions, silica, laboratory alkalinity, and TDS analyses required filling one $250-\mathrm{mL}$ polyethylene bottle with unfiltered groundwater and one $500-\mathrm{mL}$ and one $250-\mathrm{mL}$ polyethylene bottle with filtered groundwater (Wilde and others, 2004). The samples were filtered by using a $0.45-\mu \mathrm{m}$ pore-size PALL $^{\circledR}$ unvented, capsule filter that was pre-rinsed with $2 \mathrm{~L}$ of deionized water and then rinsed with $1 \mathrm{~L}$ of groundwater prior to sampling. Each $250-\mathrm{mL}$ filtered sample then was preserved with 7.5-Normal nitric acid. Samples analyzed for gross alpha and gross beta particle activities were filtered into 1-L polyethylene bottles and acidified with 7.5-Normal nitric acid. Samples for nutrient and stable isotopes of nitrogen and oxygen in dissolved nitrate analyses were collected by filtering groundwater into $125-\mathrm{mL}$ brown polyethylene bottles. Samples for the stable isotopes of carbon in dissolved inorganic carbon and carbon-14 abundance analyses were filtered and bottom filled into $500-\mathrm{mL}$ glass bottles that, first, were overfilled with three bottle volumes of filtered groundwater. These samples had no headspace and were sealed with conical caps to avoid atmospheric contamination.

For the collection of samples for radon-222 analysis, a stainless-steel and Teflon ${ }^{\circledR}$ valve assembly was attached to the sampling port at the well head (Wilde and others, 2004). The valve was closed part way to create back pressure, and a 10-mL groundwater sample was taken through a Teflon ${ }^{\circledR}$ septum on the valve assembly by using a glass syringe affixed with a stainless-steel needle. The sample was then injected into a $25-\mathrm{mL}$ vial partially filled with a scintillation mixture (mineral oil and 1,2,4-trimethylbenzene) and shaken. The vial then was placed in an insulated cardboard tube to protect the sample during shipping.

Dissolved standard gases were collected by using a copper-tube sampling apparatus attached to the hose bib at the well head. Each sample was collected by bottom filling two $150-\mathrm{mL}$ glass serum bottles that, first, were overfilled with approximately 5 gallons of unfiltered groundwater. These samples had no headspace or air bubbles inside the bottles and were sealed underwater to avoid atmospheric contamination.

Dissolved noble gases were collected in $3 / 8$-inch-diameter copper tubes by using reinforced-nylon tubing connected to the hose bib at the wellhead. Groundwater was flushed through the tubing to dislodge bubbles before the flow was restricted with a back-pressure valve. Clamps on either side of the copper tube then were tightened, trapping a sample of groundwater for analyses of dissolved noble gases (Weiss, 1968). 
Samples for analysis of the presence or absence of microbial indicators were collected at the well head by using the IDEXX Colilert ${ }^{\circledR}$ test kit following standard methods (American Public Health Association, 1998). Prior to the collection of samples, the sampling port was sterilized with isopropyl alcohol and, then, was flamed or let air dry. Groundwater was run through the sampling port for at least 2 minutes to remove any traces of the sterilizing agent. A 100 -mL Colilert ${ }^{\circledR}$ sterile sample bottle containing sodium thiosulfate (to neutralize chlorine when present) was filled directly from the sampling port. After a reagent was added, the sample bottle was inverted gently to mix the reagent with the sample. The sample was incubated for 24 hours at $35^{\circ} \mathrm{C}$ plus or minus $( \pm) 0.5^{\circ} \mathrm{C}$. Sample results were viewed against a comparator and interpreted as follows: (1) if the sample was less yellow than the comparator, then the result for the presence of total coliform and Escherichia coli (E. coli) was negative; (2) if the sample was as yellow or more than the comparator, then the result for the presence of total coliform was positive; (3) if the sample was yellow and its fluorescence was equal to or greater than that of the comparator, then the result for the presence of $E$. coli was positive. Some samples were not analyzed because the incubator temperature sensors fluctuated outside of the required temperature range during the incubation period.

Field alkalinity was measured in the mobile laboratory at the well site. Samples for field alkalinity titrations were collected by filtering groundwater into a $500-\mathrm{mL}$ polyethylene bottle. Alkalinity was measured for filtered samples by using the Gran titration method (Gran, 1952). Titration data were entered directly into the PCFF, and the concentrations of bicarbonate $\left(\mathrm{HCO}_{3}^{-}\right)$and carbonate $\left(\mathrm{CO}_{3}{ }^{2-}\right)$ were automatically calculated from the titration data by using the advanced speciation method (http://or.water.usgs.gov/alk/methods.html). Concentrations of $\mathrm{HCO}_{3}^{-}$and $\mathrm{CO}_{3}{ }^{2-}$ also were calculated from the laboratory alkalinity and $\mathrm{pH}$ measurements.

Nine laboratories performed chemical analyses for this study (appendix table A-1), although most of the analyses were done by the NWQL or by laboratories contracted by the NWQL. The NWQL maintains a rigorous QA program (Pirkey and Glodt, 1998; Maloney, 2005). Laboratory QC samples, including method blanks, continuing calibration verification standards, standard reference samples, reagent spikes, external certified reference materials, and external blind proficiency samples, are analyzed regularly. Method detection limits are tested continuously, and laboratory reporting levels are updated accordingly. The NWQL maintains National Environmental Laboratory Accreditation Program (NELAP) and other certifications (http://www.nelac-institute.org/). The USGS Branch of Quality Systems (BQS) maintains independent oversight of QA at the NWQL and laboratories contracted by the NWQL. The BQS also runs the National Field Quality Assurance Program (NFQA), which includes annual testing of all USGS field personnel for proficiency in taking field water-quality measurements (http://bqs.usgs.gov/ nfqa/). Results for analyses by the NWQL or by laboratories contracted by the NWQL were uploaded directly into NWIS. Results of analyses from other laboratories were compiled in a project database and uploaded from there into NWIS. Laboratory quality-control data were also stored in NWIS.

\section{Data Reporting}

The following section gives details about the laboratory reporting conventions and which constituents were determined by multiple methods.

\section{Reporting Limits}

The NWQL uses laboratory reporting level (LRL) and long-term method detection level (LT-MDL) as thresholds for reporting analytical results. The LT-MDL is a modification of the MDL (U.S. Environmental Protection Agency, 2002) that more thoroughly captures the effect of laboratory variability on method detection levels (Childress and others, 1999). The LT-MDL and MDL are the minimum concentration of a substance than can be measured and reported with 99-percent confidence that the concentration was greater than zero; a detection of a concentration greater than the LT-MDL or MDL has less than a 1-percent chance of being a false positive detection. The LT-MDL is calculated from analyses of at least 20 blind blank samples or from analyses of at least 20 low-concentration spiked samples completed over a period of generally 1 year, whereas the MDL is calculated from analyses of 7 low-concentration spiked samples done at one time (Childress and others, 1999). The LRL is set to minimize the reporting of false negatives (not detecting a compound when it actually is present in a sample) to less than 1 percent (Childress and others, 1999). The LRL usually is set at two times the LT-MDL.

The LRL is the primary level used by the NWQL for reporting analytical results of organic constituents. Organic constituents not detected are reported in the NWIS database as less than the associated reporting level (less than the LRL) and as non-detections in tables 5 and 6. The NWQL assigns some values "E" codes to indicate a higher level of uncertainty in the concentration. The NWQL also reports detections at concentrations less than the LT-MDL; however, these were not counted as detections in this report. Detections of VOCs at concentrations less than the LT-MDL are listed in footnote 2 in table 5. Detections of pesticides and pesticide degradates at concentrations less than the LT-MDL were not reported in tables $6 A$ and $6 B$. The LRLs for some pesticides and pesticide degradates varied from sample to sample, and the data are reported in tables $6 A$ and $6 B$ at the highest LRL. The LT-MDL and LRL values had not yet been developed for the VOC (NWQL schedules 4436 and 4437) and pesticide (NWQL schedule 2437) analytical methods used in this study, so the values listed as LT-MDLs and LRLs generally were actually short-term MDLs and interim reporting levels (IRL), respectively. The IRL is defined as two times the short-term MDL. 
The LT-MDL is generally the level used by the NWQL for reporting analytical results of inorganics, with the following exceptions: the MDL is used by the NWQL for reporting bromide, nitrate, and nitrite and by the NRP for iron species. The minimum reporting level (MRL) is used by the NWQL for reporting total dissolved solids. The MRL is the lowest measurable concentration of a constituent that can be reliably reported by using a given analytical method (Timme, 1995). Constituents not detected by the laboratories are reported in the NWIS database as less than the associated reporting level (less than LT-MDL, less than MDL, or less than MRL) and as non-detections in this report.

The reporting levels listed in this report were in effect during the period when the groundwater samples from the MACK study unit were analyzed (August 2013-April 2014). The USGS NWQL updates reporting-level values for each constituent at least once a year. The LT-MDLs for some inorganic constituents changed on October 1, 2013, and both values are reported in table $3 C$; only the greater of the two values is reported with the groundwater-quality data in tables 7 and 9. Concentrations less than the highest LT-MDL and greater than the lower LT-MDL are reported as nondetections relative to the higher LT-MDL in this report. All non-detections for inorganic constituents are reported in the NWIS database as less than the LT-MDL (except for nondetections of bromide, which are reported as less than the MDL). LT-MDL and LRL values had not yet been developed for the VOC (NWQL schedules 4436 and 4437) and pesticide (NWQL schedule 2437) analytical methods used for this study, and the data are reported by using interim reporting levels (IRL). The IRL is defined as two times the short-term MDL.

Total dissolved solids and perchlorate are reported by using minimum reporting levels (MRLs). The MRL is the least measurable concentration of a constituent that can be reliably reported by using a given analytical method (Timme, 1995).

Isotopes of hydrogen, oxygen, carbon, and nitrogen are reported by using method uncertainties (MUs). The MU generally indicates the precision of a particular analytical measurement; it gives a range of values that includes the true value.

Results for most constituents are presented by using the LRL, LT-MDL, MDL, MRL, or MU values provided by the analyzing laboratories. Results for some organic and inorganic constituents are presented by using study reporting levels (SRLs) derived from assessment of data from QC samples associated with groundwater samples collected as part of the GAMA-PBP (see the appendix section "Study Reporting Levels (SRLs) Based on Results from Previous Study Units").

The reporting limits for radiochemical constituents (radon-222, gross alpha and gross beta radioactivity, carbon-14, and tritium) are based on sample-specific critical levels ( $\mathrm{ssL}_{\mathrm{C}}$; McCurdy and others, 2008). The critical level is analogous to the LT-MDL used for reporting analytical results for organic and non-radioactive inorganic constituents. Here, the critical level is defined as the minimum measured activity that indicates a positive detection of the radionuclide in the sample with less than a 5-percent probability of a false positive detection. Sample-specific critical levels are used for radiochemical measurements because the critical level is sensitive to sample size and sample yield during analytical processing and is dependent on the instrument background, on counting times for the sample and background, and on the characteristics of the instrument used and the nuclide measured. An $\mathrm{ssL}_{\mathrm{C}}$ is calculated for each sample, and the measured activity in the sample is compared to the $\mathrm{ssL}_{\mathrm{C}}$ associated with that sample. Measured activities less than the $\mathrm{ssL}_{\mathrm{C}}$ are reported as non-detections by a dash in the data tables.

The analytical uncertainties associated with measurement of activities are also sensitive to sample-specific parameters, including sample size, sample yield during analytical processing, and the time elapsed between sample collection and various steps in the analytical procedure, as well as parameters associated with the instrumentation. Therefore, measured activities of radioactive constituents are reported with sample-specific combined standard uncertainties (CSUs). The CSU is reported at the 68-percent confidence level (1-sigma). The $\mathrm{ssL}_{\mathrm{C}}$ was not reported by the laboratory for some tritium results, in which case the CSU was used as an estimated reporting level.

\section{Notation}

Stable-isotope compositions of hydrogen, oxygen, carbon, and nitrogen are reported as relative isotope ratios in units of per mil by using the standard delta notation (Coplen and others, 2002):

$$
\delta^{i} E=\left[\frac{R_{\text {sample }}}{R_{\text {reference }}}-1\right] \times 1,000 \text { per mil }
$$

where

$i \quad$ is the atomic mass of the heavier isotope of the element,

$E \quad$ is the element ( $\mathrm{H}$ for hydrogen, $\mathrm{O}$ for oxygen, $\mathrm{C}$ for carbon, and $\mathrm{N}$ for nitrogen),

Rsample is the ratio of the abundance of the heavier isotope of the element $\left({ }^{2} \mathrm{H},{ }^{18} \mathrm{O},{ }^{13} \mathrm{C},{ }^{15} \mathrm{~N}\right)$ to the lighter isotope of the element $\left({ }^{1} \mathrm{H},{ }^{16} \mathrm{O}\right.$, ${ }^{12} \mathrm{C},{ }^{14} \mathrm{~N}$ ) in the sample, and

Rreference is the ratio of the abundance of the heavier isotope of the element to the lighter isotope of the element in the reference material. 
The reference material for hydrogen and oxygen is Vienna Standard Mean Ocean Water (VSMOW), which is assigned $\delta^{2} \mathrm{H}$ and $\delta^{18} \mathrm{O}$ values of 0 per mil (note that $\delta^{2} \mathrm{H}$ is sometimes written as $\delta \mathrm{D}$ because the common name of the heavier isotope of hydrogen, hydrogen-2, is deuterium; Coplen and others, 2002). The reference material for carbon is Vienna Pee Dee Belemnite (VPDB), which is assigned a $\delta^{13} \mathrm{C}$ value of 0 per mil (Coplen and others, 2002). The reference material for nitrogen is atmospheric nitrogen gas, which is assigned a $\delta^{15} \mathrm{~N}$ value of 0 per mil. Positive values indicate enrichment of the heavier isotope, and negative values indicate depletion of the heavier isotope compared to the ratios observed in the standard reference material.

\section{Constituents Determined by Multiple Analytical Methods}

Four constituents targeted in this study were measured by more than one analytical method. Preferred analytical methods generally were selected on the basis of performance or sensitivity for the constituent, or (in some cases) to provide consistency with historical data from the same method (U.S. Geological Survey, 2009). The constituent MTBE was analyzed as part of NWQL VOC schedules 4436 and 4437 , and only results from schedule 4437 were reported (tables $3 \mathrm{~A}$, 5). Schedule 4436 has an LRL of $0.1 \mu \mathrm{g} / \mathrm{L}$ for MTBE, whereas schedule 4437 has an LRL of $0.012 \mu \mathrm{g} / \mathrm{L}$.

The water-quality indicators $\mathrm{pH}$, specific conductance, and alkalinity were measured in the field and at the NWQL. The field measurements are generally preferred for all three constituents, and field measurements were used to describe groundwater conditions when available, although results from field and laboratory measurements were reported. The field and laboratory results were compared statistically to assess potential bias in datasets consisting of field values for some samples and laboratory values for other samples.

The field and laboratory data were compared by using the Wilcoxon signed-rank test, a nonparametric statistical test analogous to the parametric paired Student's $t$-tests (Helsel and Hirsch, 2002). A nonparametric test was used because the differences between the field and laboratory data were not normally distributed. The Wilcoxon signed-rank test evaluates the null hypothesis that the median of the paired differences between the two datasets is zero, indicating no statistical difference between the datasets. Results are reported as the probability, $p$, of obtaining the observed distribution of each dataset. Tests yielding a $p$-value of less than 0.05 were considered significant and indicated greater than 95-percent confidence that the two datasets are different.

For specific conductance, all 77 samples were measured in the field and the laboratory, and no significant differences were observed between the field and laboratory values $(p=0.83)$. The values measured in the field were used to describe and assess groundwater quality because field values were available for all samples, and they are considered more representative of groundwater conditions (Hem, 1985).

For $\mathrm{pH}$, all 77 samples were measured in the field and the laboratory, and there was a systematic difference between the field and laboratory values ( $p$ was less than 0.001). Laboratory $\mathrm{pH}$ values were greater than field $\mathrm{pH}$ values by a median of $0.3 \mathrm{pH}$ unit (standard units). Laboratory $\mathrm{pH}$ values ranged from $1.0 \mathrm{pH}$ unit more than the field $\mathrm{pH}$ values to $0.2 \mathrm{pH}$ unit less than the field $\mathrm{pH}$ values. The increase in $\mathrm{pH}$ of the groundwater samples between field and laboratory measurements can be explained by equilibration of the samples with the atmosphere after collection and by analytical methods that can introduce bias in the laboratory values (Fram and others, 2009). The field values for $\mathrm{pH}$ were used to describe and assess groundwater quality because field values were available for all samples, and field values are preferred because they are considered more representative of groundwater conditions (Hem, 1985).

For alkalinity, eight samples were measured both in the field and the laboratory. Analyzing alkalinity in the field is time-consuming and can limit the number of wells that can be visited in a single day. Laboratory alkalinity values were significantly greater than field alkalinity values $(p=0.021)$, and the median relative percentage difference was +5.4 percent.

Bennett and Fram (2014) evaluated paired field and laboratory alkalinity data using data from 616 GAMA-PBP samples collected between May 2004 and October 2012 to have sufficient data for a robust statistical assessment. In this larger dataset, laboratory alkalinity values also were significantly greater than field alkalinity values ( $p$ was less than 0.001 ), and the median relative percentage difference was +4.0 percent. The greater alkalinity values from laboratory measurements compared with those from field measurements likely were due to differences between the fixed-endpoint titration method and the Gran titration method rather than to chemical changes in the groundwater sample during the time between the two measurements (Bennett and Fram, 2014). Equilibration of the sample with the atmosphere by loss or gain of carbon dioxide does not change alkalinity (although $\mathrm{pH}$ and dissolved inorganic carbon concentration change; Stumm and Morgan, 1996).

The systematic difference between the field and laboratory alkalinity values is unlikely to affect interpretation of the chemical data because the difference is too small to significantly disrupt the cation-anion balances. Among the 616 samples examined by Bennett and Fram (2014), 70 percent had a cation-balance within plus or minus 2 percent using the laboratory alkalinity values, and 67 percent had a cation-balance within plus or minus 2 percent using the field alkalinity values. Ninety-seven percent had a cation-balance within plus or minus 5 percent using either alkalinity value. These results indicated that the field and laboratory alkalinity values both yield high-quality major-ion data. 


\section{Quality-Control Methods and Results}

The purpose of QC is to identify which data represent environmental conditions best and which could have been affected by contamination or bias during sample collection, processing, storage, transportation, or laboratory analysis. Four types of QC measurements were evaluated in this study: (1) blank samples were collected to assess positive bias as a result of contamination during sample handling or analysis, (2) replicate samples were collected to assess variability, (3) matrix-spike tests were done to assess positive or negative bias, and (4) surrogate compounds were added to samples analyzed for organic constituents to assess potential bias of laboratory analytical methods.

\section{Blanks}

The primary purposes of collecting blanks are to evaluate the potential contamination of samples with compounds of interest during sample handling or analysis and to identify and mitigate the sources of sample contamination. Results from blanks collected for the MACK study unit and for previous GAMA study units were used to establish study reporting levels (SRLs) for some constituents detected in blanks. SRLs have higher concentrations than the reporting levels used by the laboratory and are used as raised reporting levels. Detections of organic constituents reported by the laboratory at concentrations less than these raised reporting levels are considered non-detections in this study. Detections of inorganic constituents in groundwater samples at concentrations less than SRLs have been reclassified with a "less than or equal to" symbol preceding the reported value to indicate that the true value could be less than or equal to the reported value (including the possibility of being a nondetection) by the laboratory. These data were flagged with an appropriate remark code (described in subsequent sections).

\section{Blank Collection and Analysis}

Blanks were collected by using blank water certified by the NWQL to contain concentrations less than the reporting levels for selected constituents investigated in the study (James A. Lewis, National Water Quality Laboratory, written commun., 2014). Nitrogen-purged, organic-free blank water was used for blanks processed for the evaluation of organic constituents, and inorganic-free blank water was used as the blank water for inorganic constituents. Sterile water (deionized water autoclaved at the USGS California Water Science Center) was used for the collection of field blanks for bacteria. Three types of blanks were collected for the MACK study: source-solution blanks, field blanks, and an equipment blank.
Source-solution blanks were collected when using a new lot of blank water to assess potential contamination of samples during transport and analysis or potential contamination of the certified blank water obtained from the NWQL. A "lot" is a unique batch of blank water with defined production and expiration dates. Source-solution blanks were typically collected in the field inside the mobile laboratory by pouring blank water directly into sample containers that were preserved, stored, shipped, and analyzed in the same manner as the groundwater samples. Source-solution blanks were analyzed for VOCs, trace elements, major and minor ions, silica, TDS, and perchlorate.

One equipment blank was collected at the beginning of the study to assess the status of the Grundfos pump apparatus before it was used to collect samples from the six monitoring wells sampled for this study. This equipment blank was collected by using the Grundfos pump and an attached reel of sampling line to pump blank water from a container into the sampling bottles. An additional source-solution blank was collected on the same day.

Field blanks were collected to assess potential contamination of samples during collection, processing, transport, and analysis. To collect field blanks at the sampling sites, blank water either was pumped or poured through the sampling equipment (fittings and tubing) used to collect groundwater samples and, then, was processed and transported following the same protocols used for the groundwater samples. Four liters of blank water were pumped or poured through the sampling equipment before each field blank was collected. Field blanks were analyzed for VOCs, pesticides and pesticide degradates, trace elements, nutrients, major and minor ions, silica, TDS, and perchlorate. Field blanks were also collected for bacteria samples. To collect field blanks for bacteria at the sampling sites, sterilized water was poured directly into a similar type of sample bottle as was used to collect groundwater samples, then was processed and transported following the same protocols used for the groundwater samples.

Field or source-solution blanks were not collected for radon-222 or gross alpha and gross beta radioactivity because the laboratory determines an $\mathrm{ssL}_{\mathrm{C}}$ value for each sample. The $\mathrm{ssL}_{\mathrm{C}}$ is the minimum measured value that indicates a non-zero amount of the radionuclide in the sample; in other words, it is an amount of the radionuclide that is statistically significantly greater than the amount in a blank. Blanks were not collected for tritium and dissolved noble gases. Tritium and dissolved noble gases are in the atmosphere and dissolve into any solution used for collecting a blank, making it impractical to collect a blank for these analytes. Stable isotopes of hydrogen, oxygen, carbon, and nitrogen are an intrinsic property of any of these elements; therefore, the concept of a blank does not apply to these ratios. 


\section{Study Reporting Levels (SRLs) Based on Results from Previous Study Units}

The SRLs for VOCs were determined by Fram and others (2012) on the basis of statistical assessment of results from field blanks, source-solution blanks, laboratory instrument blanks, and groundwater samples collected for GAMA-PBP study units sampled May 2004 through September 2010. The SRLs were established for those VOCs that had evidence of potential contamination due to field or laboratory processes: 1,2,4-trimethylbenzene, carbon disulfide, chloroform, ethylbenzene, toluene, $m$ - plus $p$-xylenes, $o$-xylene, acetone, 2-butanone, and tetrahydrofuran (Fram and others, 2012). In the groundwater sampled from the MACK study unit, concentrations of toluene were reported in two samples; both were less than the SRL of $0.69 \mu \mathrm{g} / \mathrm{L}$, were not counted as detections, and thus were only reported in the footnote of table 5. Four detections of chloroform and three detections of carbon disulfide at concentrations less than the SRL also were at concentrations less than the LT-MDL; thus, application of the SRL was not needed, and these reported concentrations were not counted as detections (table 5).

The SRLs for trace elements were determined on the basis of statistical assessments of results from field blanks, source-solution blanks, and laboratory blanks collected for GAMA-PBP study units May 2004 through March 2013 (Olsen and others, 2010; Davis and others, 2014). These assessments used order statistics and binomial probabilities to construct an upper confidence limit (Hahn and Meeker, 1991) for the maximum concentration of each constituent potentially introduced while groundwater samples were collected, handled, transported, and analyzed. The SRLs from Davis and others (2014) apply to the period during which samples were collected for the MACK study unit: copper $(2.1 \mu \mathrm{g} / \mathrm{L})$, iron $(6 \mu \mathrm{g} / \mathrm{L})$, lead $(0.82 \mu \mathrm{g} / \mathrm{L})$, manganese $(0.66 \mu \mathrm{g} / \mathrm{L})$, molybdenum $(0.023 \mu \mathrm{g} / \mathrm{L})$, nickel $(0.21 \mu \mathrm{g} / \mathrm{L})$, and zinc $(6.2 \mu \mathrm{g} / \mathrm{L})$. Detections of these trace elements at concentrations less than the SRLs are marked with a "less than or equal to" symbol preceding the reported value in table 7 to indicate that the true value could be less than or equal to the reported value (including the possibility of being a non-detection). One additional trace element - cobalt — had no upper limit on the concentrations that could be attributed to extrinsic contamination (Davis and others, 2014). All groundwater results for cobalt were coded as "reviewed and rejected" in the NWIS database and are not presented in this report.

\section{SRLs Based on Detections in S3-MACK Blanks}

Appendix table A-2 presents a summary of detections in the blanks (seven field blanks, three source-solution blanks, and one equipment blank) and the SRLs that were applied for the MACK study unit. Field blanks were collected at 9 percent of the sites sampled in the MACK study unit. One additional field blank was collected for the analysis of bacteria, making a total of eight bacteria field blanks.

Four VOCs (cyclohexanone, 1,1-difluoroethane, isopropyl alcohol, and tert-butyl alcohol) were detected in blanks collected for the MACK study unit (appendix table A-2). An SRL was not defined for three of these VOCs: cyclohexanone was reported in one groundwater sample at a concentration below the LT-MDL; 1,1-difluoroethane was not detected in any groundwater samples; and tert-butyl alcohol was only detected at a concentration greater than the detection in the field blank in one groundwater sample. The concentration of isopropyl alcohol detected in a field blank was greater than that in two groundwater samples. Because of the detections in the blanks and unacceptable results for replicates (see "Replicates" section and appendix table A-3A), all five reported concentrations of isopropyl alcohol in groundwater samples were considered suspect and were not counted as detections (table 5, appendix table A-2).

Four pesticides and pesticide degradates were detected in field blanks (appendix table A-2). Three of the compounds were not detected in groundwater samples. All detections of simazine in groundwater samples were at concentrations greater than that detected in the field blank; thus, no SRL was needed.

Eight trace elements were detected in blanks collected for the MACK study unit (appendix table A-2). For cobalt, copper, lead, manganese, nickel, and zinc, the SRLs defined by Davis and others (2014) were applied to the data. Detections of these trace elements at concentrations below their respective SRLs were flagged with a "less than or equal to" symbol (table 7). No SRLs were defined for chromium or molybdenum because the detections in blanks were at lower concentrations than the minimum concentration detected in groundwater samples (appendix table A-2). In addition, these two trace elements were only detected in the blank for the Grundfos pump, and this equipment was only used to collect the six groundwater samples from monitoring wells.

Ammonia was detected in three of the seven field blanks at concentrations equal to and slightly greater than the LT-MDL (table 8, appendix table A-2). Concentrations of ammonia detected in groundwater samples were greater than the detections in the field blanks; therefore, no SRL was necessary (table 8, appendix table A-2).

Silica was detected in one of the seven field blanks at a concentration of $0.053 \mathrm{mg} / \mathrm{L}$, but concentrations of silica detected in groundwater samples were greater than that detected in the field blank, so no SRL was needed (table 9, appendix table $\mathrm{A}-2$ ). 


\section{Interim Study Reporting Levels Based on Laboratory Blank Data}

Some pesticides and pesticide degradates in the newly established analytical method (NWQL schedule 2437) were detected in laboratory prep blanks. The QC data from previous GAMA study units were not available because this study unit was the first to use the method. Laboratory prep blanks were reviewed to determine if an interim study reporting level (iSRL) would be needed to raise laboratory reporting levels and further reduce the probability of reporting false-positive detections. An iSRL was defined from the examination of about 150 to 200 laboratory prep blanks for each constituent analyzed from 2013 through 2015 if the 99th percentile value of the blanks was greater than the lowest concentration detected in a groundwater sample and was greater than the highest LT-MDL. The iSRL was set equal to the 99th percentile value of the laboratory prep blanks, following the detection limit by blank data reporting described in the NWQL Technical Memorandum 15.02 (National Water Quality Laboratory, 2015). Out of the 229 pesticides and pesticide degradates analyzed using the NWQL schedule 2437, an iSRL was determined for four pesticides: atrazine, fipronil, methoxyfenozide, and DCPMU (table 3B). Detections at concentrations less than the respective iSRLs were not reported and were not counted as detections for the purposes of calculating detection frequencies in tables $6 A$ and $6 B$.

\section{Replicates}

Sequential replicate samples were collected to assess the precision of the water-quality data. Estimates of data precision are needed to assess whether differences between concentrations in samples are due to differences in groundwater quality or to variability that can result from collecting, processing, and analyzing the samples.

\section{Assessment of Replicate Samples}

Two methods for measuring variability were needed to adequately assess precision for the broad range of measured concentrations of most constituents. The variability between measured concentrations in the pairs of sequential replicate samples was represented by the standard deviation (SD) for low concentrations and by relative standard deviation (RSD) for high concentrations (Anderson, 1987; Mueller and Titus, 2005). The RSD is defined as the SD divided by the mean concentration of each replicate sample pair, expressed as a percentage. The boundary between concentrations for which variability is assessed with SDs and concentrations for which variability is assessed with RSDs was defined as five times the reporting level (RL) for each constituent. For VOCs and pesticides, the LRL was used as the RL to determine whether a pair would be evaluated by using the RSD or the SD. For trace elements, nutrients, and major ions, the LT-MDL was used as the RL to determine whether a pair would be evaluated by using the RSD or the SD. If a constituent had an SRL, the SRL was used instead of the LT-MDL. For isotopic ratios, pairs were evaluated by using the RSD.

Replicate sample pairs for all constituents, except for radiochemical constituents, were evaluated as follows:

- If both values were reported as detections, the SD was calculated if the mean concentration was less than 5 RL for the constituent, and the RSD was calculated if the mean concentration was greater or equal to $5 \mathrm{RL}$ for the constituent. Acceptable precision was defined as an SD of less than one-half the RL or an RSD of less than 10 percent.

- If both values were reported as non-detections, the variability was set to zero by definition.

- If one value was reported as a non-detection and the other value was reported as a detection, then a value of zero was substituted for the non-detection, and the SD was calculated. Substituting zero for the non-detection yielded the maximum estimate of variability for the replicate sample pair.

- If one value for a replicate sample pair was reported as a non-detection and the other value was reported as less than or equal to the SRL (a coded value), or if both values were reported as coded values of less than or equal to the SRL, the SD was not calculated because the values could be analytically identical. The "less than or equal to" code indicates that the associated value is a maximum potential concentration, and that concentration could be low enough to be reported as a non-detection.

Replicate samples of radiochemical constituents were evaluated by using the following equation (McCurdy and others, 2008) to calculate the normalized absolute difference (NAD):

$$
N A D=\frac{\left|R_{1}-R_{2}\right|}{\sqrt{\left(C S U_{1}^{2}+C S U_{2}^{2}\right)}}
$$

$$
\begin{aligned}
& \begin{array}{l}
\text { where } \\
R_{1} \text { and } R_{2} \quad \text { are the results for the two samples in the } \\
\text { replicate sample pair, and }
\end{array} \\
& C S U_{1} \text { and } C S U_{2} \text { are the combined standard uncertainties } \\
& \text { associated with the results. }
\end{aligned}
$$

A value of 1.65 for the NAD corresponds to a significance level $(\alpha)$ of 5 percent $(\alpha=0.05)$. Values of NAD less than 1.65 were defined as corresponding to differences that are not statistically different, and which, therefore, indicate acceptable replicate results. 
If results from replicate samples indicate that precision was unacceptable for a constituent and no specific cause was identified, then this greater variability must be considered when interpreting the data. For example, if measured concentrations are slightly greater than a water-quality benchmark, then actual concentrations could be slightly less than that benchmark. Similarly, if measured concentrations are slightly less than a water-quality benchmark, then actual concentrations could be slightly greater than a benchmark. Also, if a constituent has high variability in replicate samples, then a greater difference between concentrations measured in two samples is required to conclude that the two samples have concentrations that are meaningfully different.

\section{Variability in Replicate Samples}

Appendix tables A-3A-C summarize the results of replicate samples for constituents detected in groundwater samples collected in the MACK study unit. Replicate samples were collected at seven wells, representing approximately 9 percent of all the samples collected. Approximately 2,700 replicate pairs of constituent determinations resulted, and of those, about 300 pairs had a detection in one or both samples of the pair.

Of these 300 pairs, 7 pairs - one each for 7 constituents (carbon disulfide, isopropyl alcohol, cadmium, copper, nitrite, gross beta radioactivity for 72 -hour count, and tritium) - were outside the limits for acceptable precision. Results for replicate samples of constituents that were not detected in groundwater samples are not reported in appendix tables A-3A-C. All replicate samples for alkalinity, major and minor ions, perchlorate, isotopic tracers, and microbial indicators had variability within acceptable precision.

For each of the 90 VOCs, 7 replicate samples were analyzed. Of the 630 replicate pairs, 623 were composed of 2 values reported as non-detections. Of the six replicate pairs with at least one detection, four were paired detections of 1,2,3-trichloropropane, 1,2-dichloropropane, or 1,2-dibromo3 -chloropropane, and all four pairs had acceptable precision (appendix table A-3A). Carbon disulfide and isopropyl alcohol each had a replicate pair consisting of a non-detection and a detection with a concentration greater than the LRL. Both pairs were considered unacceptable precision. There were no other quality-control issues with carbon disulfide, and no action was taken on the basis of this unacceptable precision. The maximum concentration of carbon disulfide detected in groundwater samples $(0.21 \mu \mathrm{g} / \mathrm{L})$ was far less than the HBSL benchmark concentration $(700 \mu \mathrm{g} / \mathrm{L})$; thus, the less than acceptable precision was considered unlikely to affect interpretation of the data. Isopropyl alcohol also was detected in a field blank at a concentration greater than the concentration in two of the groundwater samples. On the basis of these two quality-control issues, all detections of isopropyl alcohol in groundwater samples were considered suspect, and the data were censored.

For each of the 229 pesticides and pesticide degradates, 7 replicate samples were analyzed. Of the 1,603 replicate pairs, 1,598 were composed of 2 values reported as nondetections. Of the 15 replicate pairs with at least one detection, 14 pairs had acceptable precision (appendix table A-3A). The pesticide degradate compound hlorodiamino-s-triazine (CAAT) had a replicate pair with unacceptable precision; however, the concentrations were less than five times the LRL.

Seven replicate samples were analyzed for each of the nutrients, trace elements, and major ions. Of the 294 replicate pairs, only 73 were composed of 2 values reported as nondetections in this study. Of the 221 replicate pairs composed of at least 1 detection, 217 had acceptable precision (appendix table A-3A). The four pairs with unacceptable precision each were composed of a non-detection and a detection at a concentration less than five times the LT-MDL (nitrite-N, cadmium, copper, and nickel, appendix table A-3A).

Seven replicate samples of each isotopic tracer were analyzed for variability, and all the RSDs were within acceptable precision (appendix tables A-3A, A-3B).

The seven replicate samples of each radioactive constituent (including tritium) were analyzed for variability. All replicated samples yielded statistically similar results ( $p$ less than or equal to 0.05 ), with two exceptions: one replicate sample pair for gross beta radioactivity (72-hour count) and one replicate sample pair for tritium (appendix table A-3B).

Seven replicate samples of each microbial indicator (E. coli, total coliform, and enterococci) were analyzed for variability, and all results were within acceptable precision (appendix table A-3C). All replicate pairs were composed of two samples that had no verifiable presence of microbial indicators, except for one replicate pair for which the presence of total coliform was detected in both samples composing the pair.

\section{Matrix Spikes}

A matrix-spike pair consists of two environmental samples from the same well to which a known concentration of constituents has been added to one of the two samples. Matrix-spike pairs are used to evaluate the effect of the matrix, in this case groundwater, on the analytical technique used to measure the constituent. The known compounds added in matrix spikes are the same as those analyzed in the environmental samples. This enables an analysis of matrix interferences on a compound-by-compound basis. For this study, matrix spikes were added by the laboratory performing the analysis rather than in the field. Low matrix-spike recovery can indicate that the compound would not be detected in some samples if present only at very low concentrations. Low and high matrix-spike recoveries can be a potential concern if the concentration of a compound in a groundwater sample is close to the health-based benchmark; a low recovery could result in a falsely measured concentration less than the healthbased benchmark, whereas a high recovery could result in a falsely measured concentration greater than the health-based benchmark. 
The GAMA-PBP defined the data-quality objective range for acceptable median matrix-spike recoveries at 70 to 130 percent. Only constituents that had median matrix-spike recoveries outside of this range were flagged for unacceptable recoveries. For some constituents, an acceptable range of 70 to 130 percent for the median matrix-spike recovery was more restrictive than the acceptable control limits for laboratoryset spike recoveries. Laboratory-set spikes are aliquots of laboratory blank water to which the same spike solution used for the matrix spikes has been added. One set spike is analyzed for each set of samples. Acceptable control limits for set spikes are defined relative to the long-term variability in recovery. For example, for many NWQL analyses, acceptable set-spike recovery is within $\pm 3 \mathrm{~F}$-pseudosigma of the median recovery for at least 30 set spikes (Conner and others, 1998). The F-pseudosigma is calculated by dividing the fourth-spread (analogous to interquartile range) by 1.349; therefore, the less the F-pseudosigma, the more precise the determinations (Hoaglin, 1983).

Matrix spikes were performed for VOCs, pesticides, and pesticide degradates because the analytical methods for these constituents can be susceptible to matrix interferences.

Appendix tables A- $4 A-B$ present a summary of matrixspike recoveries for the MACK study unit. Replicate samples for spike additions of organic constituents were collected at six wells for VOCs, pesticides, and pesticide degradates, which was approximately 8 percent of all the wells sampled.

Groundwater samples were spiked with 89 VOCs to calculate matrix-spike recoveries (the laboratory did not report the expected spike concentration for butanol). Median matrix-spike recoveries were between 70 and 130 percent for all VOCs, with the exception of butane, which had a median matrix-spike recovery of 65 percent (appendix table A-4A). Low matrix-spike recovery could indicate that the compound would not be detected in some samples if only present at very low concentrations. Butane was detected in three groundwater samples ranging in concentration from $0.020 \mu \mathrm{g} / \mathrm{L}$ to $0.041 \mu \mathrm{g} / \mathrm{L}$ (table 5). There is no benchmark for butane. The minimum matrix-spike recovery was less than 70 percent for 10 VOCs, and the maximum matrix-spike recovery was greater than 130 percent for 3 VOCs.

Groundwater samples were spiked with 228 pesticides and pesticide degradates to calculate matrix-spike recoveries (matrix-spike samples were not analyzed for phosmet; appendix table A-4B). Median matrix-spike recoveries were between 70 and 130 percent for 196 of the 228 pesticides and pesticide degradates. Of the 31 pesticides and pesticide degradates detected in samples from the MACK study unit, only one, 1H-1,2,4-triazole, had a median matrix-spike recovery outside of the acceptable range. The minimum matrix-spike recovery was less than 70 percent for 227 pesticides and pesticide degradates, and the maximum matrix-spike recovery was greater than 130 percent for 31 pesticides and pesticide degradates. This large variability in matrix-spike recoveries indicates that the analytical method does not reliably measure concentrations, and that the method primarily is biased towards reporting lower concentrations than are actually present in samples.

Environmental detections were not modified on the basis of the matrix-spike recovery analysis.

\section{Surrogates}

Surrogate compounds are added to environmental samples in the laboratory prior to analysis to evaluate the recovery of similar constituents. Surrogate compounds were added in the laboratory to all groundwater and QC samples that were analyzed by the NWQL for VOCs, pesticides, and pesticide degradates. Surrogates are used to identify general problems that could arise during laboratory sample analysis that can affect the results for all compounds in that sample. Potential problems include matrix interferences (such as high levels of dissolved organic carbon) that produce a positive bias or incomplete laboratory recovery (possibly because of improper maintenance and calibration of analytical equipment) that produces a negative bias. A 70 to 130 percent recovery of surrogates, in general, is considered acceptable; values outside this range indicate possible problems with the processing and analysis of samples (Connor and others, 1998; Sandstrom and others, 2001).

Surrogate recoveries from the 9 field, equipment, or source-solution blanks were compared to surrogate recoveries from the 77 groundwater samples and the 7 replicates. Wilcoxon rank-sum tests indicated no significant differences between surrogate recoveries in blanks compared to groundwater samples for 23 of the 26 surrogate compounds ( $p$-values ranged from 0.075 to 0.944 ). For cis-permethrin$13 \mathrm{C} 6$ and alachlor- $d 13$, surrogate recoveries for blanks were significantly greater than surrogate recoveries for groundwater samples, and for carbendazim- $d 4$, surrogate recoveries for groundwater samples were significantly greater than surrogate recoveries for blanks. These results indicated that there could be some matrix characteristics of groundwater samples that could affect recovery of some pesticides and pesticide degradates. Because the recoveries for cis-permethrin-13C6, alachlor- $d 13$, and carbendazim- $d 4$ were within the acceptable range for 89,92 , and 88 of the 92 samples, respectively, however, the difference between surrogate recoveries in blanks and groundwater samples was not considered great enough to be meaningful.

Appendix table A-5 lists the minimum, 10th percentile, 25th percentile, median, 75th percentile, 90 th percentile, and maximum recovery for each of the 26 surrogate compounds. The 10th-percentile recovery value was greater than 70 percent for all 26 surrogate compounds, and only 4 surrogate compounds had a minimum recovery less than 70 percent. The 90th-percentile recovery was less than 130 percent for 24 of the 26 surrogate compounds, and only 4 surrogate compounds had a maximum recovery of greater than 130 percent. These results indicated that surrogate recoveries were generally acceptable.

Environmental detections were not modified on the basis of the surrogate recovery analysis. 


\section{Other Quality-Control Issues}

Three other laboratory QC issues arose during the analysis of samples collected for the MACK study unit: the effect of holding-time violations on the results for radioactive constituent data, the effect of laboratory bias on the results for trace element data, and the rejection of analytical data.

\section{Effect of Holding-Time Violations}

Holding time refers to the recommended maximum time in calendar days from sample collection to the analysis of the sample. A holding-time violation is committed when a sample is analyzed past the designated holding time for a particular analysis. Analyses of some samples in the MACK study unit were completed after the holding time because of a scheduling error at the laboratory or shipping error. A delay in the analysis at the laboratory can result in different measured activities or concentrations than what was present in the sample.

The gross alpha- and beta-particle results are reported as the amount of activity measured in the sample. Because of decay of radioactive elements present in the groundwater sample at the time of collection and ingrowth of radioactive daughter products, gross alpha- and gross beta-particle activities can change over time. Gross alpha- and beta-particle activities for 72-hour counts were analyzed past the holding time in 11 groundwater samples, and gross beta-particle activities for 72-hour counts were analyzed past the holding time in 4 additional groundwater samples. The results for these samples are footnoted in table 11. A replicate sample was analyzed past the holding time for one sample collected for 72-hour counts of gross alpha- and beta-particle activities, but the precision was within acceptable ranges. Gross alphaparticle activity (72-hour) could be less, and gross betaparticle activity (72-hour) can be less or more, in samples analyzed past the holding time than if they had been analyzed on time.

\section{Laboratory Bias}

Laboratory bias indicated from internal laboratory QC tests is another issue that must be investigated to determine whether the data are affected. The Branch of Quality Systems (BQS) operates independent, external quality-assurance projects called the Inorganic Blind Sample Project (IBSP) and Blind Blank Program (BBP) to monitor and evaluate the quality of results for analyses of trace elements, nutrients, major and minor ions, silica, and TDS by the NWQL. The IBSP submits standard-reference samples consisting of natural matrix water samples spiked with reagent chemicals to contain known concentrations of the inorganic constituents (Farrar and Long, 1997). The IBSP samples are disguised as regular environmental samples for submission to the NWQL; the BBP samples are disguised as regular blank samples. The $\mathrm{BQS}$ uses results from the IBSP and BBP samples to evaluate potential bias in the results reported by the NWQL. The BQS summarizes results every other month, reporting the amount of bias (if any) observed in the results.

Samples from the S3-MACK study unit were analyzed between August 2013 and April 2014. Results from BQS samples analyzed during the same period were reported in the October 2013 through June 2014 BQS summary reports. The BQS reported that five inorganic constituents showed evidence of bias during this period: positive bias for laboratory alkalinity and fluoride, and negative bias for arsenic, boron, and lead (Tedmund M. Struzeski, U.S. Geological Survey Branch of Quality Systems, written commun., 2014). The BQS determines bias from the relative percentage difference between the measured and most probable (known) values.

The ISBP data for these five constituents were examined to determine whether the laboratory bias was large enough to affect the results of comparisons between concentrations in environmental samples and benchmark concentrations. The IBSP data were evaluated using an approach similar to that used to evaluate replicate analyses for this study. For each constituent, the IBSP samples were divided into two groups of most probable concentrations greater than and less than a threshold value equal to five times the reporting limit. Bias in the samples that had concentrations above the threshold value was quantified by using relative percentage difference between the measured and most probable values, whereas bias in the samples that had concentrations below the threshold value was quantified by using the absolute difference between the measured and most probable values.

Alkalinity concentrations in the 46 IBSP samples from the October 2013 through June 2014 BQS summary reports ranged from 13 to $74 \mathrm{mg} / \mathrm{L}$ as calcium carbonate $\left(\mathrm{CaCO}_{3}\right)$. The 36 IBSP samples that had alkalinity concentrations less than $40 \mathrm{mg} / \mathrm{L}$ as $\mathrm{CaCO}_{3}$ (five times the reporting limit of $8 \mathrm{mg} / \mathrm{L}$ as $\mathrm{CaCO}_{3}$ ) had a median bias of $+2.3 \mathrm{mg} / \mathrm{L}$ as $\mathrm{CaCO}_{3}$, and the 10 IBSP samples that had alkalinity concentrations greater than $40 \mathrm{mg} / \mathrm{L}$ had a median bias of +3.4 percent. The measured value was greater than the most probable value for all 46 samples, and the magnitude of positive bias was similar to the magnitude of positive bias in laboratory alkalinity values compared to field alkalinity values. As discussed in the section "Constituents determined by multiple analytical methods," this bias was unlikely to cause data-quality problems.

Arsenic concentrations in the 55 IBSP samples from the October 2013 through June 2014 BQS summary reports ranged from 0.18 to $18.7 \mu \mathrm{g} / \mathrm{L}$. The 26 IBSP samples that had arsenic concentrations less than $1.0 \mu \mathrm{g} / \mathrm{L}$ (10 times the reporting limit) had a median bias of $-0.01 \mu \mathrm{g} / \mathrm{L}$ (interquartile range, IQR, was -0.06 to $+0.02 \mu \mathrm{g} / \mathrm{L}$ ), and the 29 IBSP samples that had arsenic concentrations greater than $1.0 \mu \mathrm{g} / \mathrm{L}$ had a median bias of -0.1 percent (IQR was -7.5 to +2.2 percent). The median bias values in both concentration groups were negative, but close to zero, and the IQRs of the bias values straddled zero bias. Therefore, the bias was not considered sufficiently systematic to cause data-quality concerns. 
Boron concentrations in the 51 IBSP samples from the October 2013 through June 2014 BQS summary reports ranged from 5.6 to $37 \mu \mathrm{g} / \mathrm{L}$. The 33 IBSP samples that had boron concentrations less than $25 \mu \mathrm{g} / \mathrm{L}$ (five times the reporting limit) had a median bias of $+0.3 \mu \mathrm{g} / \mathrm{L}$ (IQR was -0.2 to $+1.1 \mu \mathrm{g} / \mathrm{L}$ ), and the 18 IBSP samples that had boron concentrations greater than $25 \mu \mathrm{g} / \mathrm{L}$ had a median bias of +3.1 percent (IQR was -0.6 to +5.1 percent). The median bias values in both concentration groups were positive, but the IQRs of the bias values straddled zero bias. Therefore, the bias was not considered sufficiently systematic to cause dataquality concerns.

Fluoride concentrations in the 56 IBSP samples from the October 2013 through June 2014 BQS summary reports ranged from 0.05 to $0.63 \mathrm{mg} / \mathrm{L}$. The $11 \mathrm{IBSP}$ samples that had fluoride concentrations less than $0.1 \mathrm{mg} / \mathrm{L}$ (10 times the reporting limit) had a median bias of $+0.010 \mathrm{mg} / \mathrm{L}$ (IQR was +0.006 to $+0.014 \mathrm{mg} / \mathrm{L}$ ), and the 45 IBSP samples that had fluoride concentrations greater than $0.1 \mathrm{mg} / \mathrm{L}$ had a median bias of +6.3 percent (IQR was +4.8 to +7.7 percent). These results indicated that fluoride concentration results likely had a positive bias. Of the 77 samples in the MACK study unit, only 2 had fluoride concentrations greater than $0.5 \mathrm{mg} / \mathrm{L}$ (S3-MACK-K02, 1.05 mg/L; S3-MACK-K13, 1.07 mg/L). If the bias observed in the IBSP samples is assumed to apply to concentrations outside of the concentration range of the IBSP samples, then the two MACK study-unit samples that had measured concentrations just more than one-half the value of the MCL-CA for fluoride actually could have had concentrations just less than one-half the MCL-CA (table 7).

Lead concentrations in the 55 IBSP samples from the October 2013 through June 2014 BQS summary reports ranged from 0.29 to $56 \mu \mathrm{g} / \mathrm{L}$. The 47 IBSP samples that had lead concentrations less than $4 \mu \mathrm{g} / \mathrm{L}$ (100 times the reporting level) had a median bias of $-0.07 \mu \mathrm{g} / \mathrm{L}$ (IQR was -0.14 to $-0.02 \mu \mathrm{g} / \mathrm{L}$ ), and the 8 IBSP samples that had lead concentrations greater than $4 \mu \mathrm{g} / \mathrm{L}$ had a median bias of +0.5 percent (IQR was -1.3 to +3.4 percent). The median bias value in the high concentration group was close to zero, and the IQR of the bias value straddled zero. Therefore, the bias was not considered sufficiently systematic to cause data-quality concerns. Low concentrations of lead appeared to have a slight systematic negative bias. The maximum concentration of lead detected in groundwater samples was $1.69 \mu \mathrm{g} / \mathrm{L}$, which was much less than the AL-US benchmark concentration of $15 \mu \mathrm{g} / \mathrm{L}$ (table 7); therefore, a slight negative bias in concentrations was unlikely to affect interpretation of the water-quality data.

\section{Rejected Analytical Data}

Analytical results for pesticides and pesticide degradates in the NWQL analytical schedule 2437 were reviewed and rejected for one well (S3-MACK-M20) and are not reported in tables $6 A$ or $6 B$ because results were considered suspect and analyses could not be re-rerun for verification due to an initial delay in processing. Concentrations of 31 pesticides and pesticide degradates were reported by the laboratory in the sample from this well and results were considered suspect because of the combination of the large number of reported concentrations in combination with other information for the well (absence of VOC detections, nitrate concentration of less than $2.5 \mathrm{mg} / \mathrm{L}$, tritium activity of less than $2 \mathrm{pCi} / \mathrm{L}$, and a well depth of 280 feet below land surface).

Analytical results for the nutrient phosphate reported under the USGS parameter code 00671 in the NWQL analytical schedule 2755 were reviewed and rejected for three wells (S3-MACK-M10, S3-MACK-M15, and S3-MACK-M20) and are reported as not available in table 8 because results were considered suspect. Concentrations greater than $10 \mathrm{mg} / \mathrm{L}$ of phosphate were reported by the laboratory for the samples from these wells, and results were considered suspect because these values were greater than any concentration reported to date (November 2016) in the USGS GAMA data for this method and because the resulting ion composition failed quality-assurance checks. The cationanion imbalances for two of the three samples were outside of the acceptable range of plus or minus 5 percent: both had high negative imbalances, indicating an excess of anions in the ion composition (phosphate is an anion). In addition, equilibrium calculations showed all three samples would be greatly oversaturated with hydroxyapatite, indicating that such high phosphate concentrations are improbable. The three wells were sampled consecutively, and the samples were analyzed in the same batch by the laboratory.

\section{References Cited}

American Public Health Association, 1998, Standard methods for the examination of water and wastewater (20th ed.): Washington D.C., American Public Health Association, American Water Works Association, and Water Environment Federation, p. 3-37-3-43.

American Society for Testing and Materials, 1998, Water and Environmental Technology, in Annual Book of ASTM Standards: Philadelphia, Pa., American Society for Testing and Materials, section 11.02 (Water II), p. 664-666.

Anderson, R.L., 1987, Practical statistics for analytical chemists: New York, Van Nostrand Reinhold Company, Inc., $315 \mathrm{p}$.

Bennett, G.L., and Fram, M.S., 2014, Groundwater-quality data in the North San Francisco Bay shallow aquifer study unit, 2012-Results from the California GAMA Program: U.S. Geological Survey Data Series 865, p. 94., http://dx.doi.org/10.3133/ds865. 
Bullen, T.D., Krabbenhoft, D.P., and Kendall, Carol, 1996, Kinetic and mineralogic controls on the evolution of groundwater chemistry and ${ }^{87} \mathrm{Sr} /{ }^{86} \mathrm{Sr}$ in a sandy silicate aquifer, northern Wisconsin, USA: Geochimica et Cosmochimica Acta, v. 60, no. 10, p. 1807-1821, http://dx.doi.org/10.1016/0016-7037(96)00052-X.

Busenberg, Eurybiades, Plummer, L.N., and Bartholomay, R.C., 2001, Estimated age and source of the young fraction of ground water at the Idaho National Engineering and Environmental Laboratory: U.S. Geological Survey Water-Resources Investigations Report 2001-4265, 144 p., https://pubs.er.usgs.gov/publication/wri014265.

Childress, C.J.O., Foreman, W.T., Connor, B.F., and Maloney, T.J., 1999, New reporting procedures based on long-term method detection levels and some considerations for interpretations of water-quality data provided by the U.S. Geological Survey National Water Quality Laboratory: U.S. Geological Survey Open-File Report 99-193, 19 p., https://pubs.er.usgs.gov/publication/ofr99193.

Connor, B.F., Rose, D.L., Noriega, M.C., Murtagh, L.K., and Abney, S.R., 1998, Methods of analysis by the U.S. Geological Survey National Water Quality Laboratory-Determination of 86 volatile organic compounds in water by gas chromatography/mass spectrometry, including detections less than reporting limits: U.S. Geological Survey Open-File Report 97-829, 78 p., https://pubs.er.usgs.gov/publication/ofr97829.

Coplen, T.B., 1995, Reporting of stable hydrogen, carbon, and oxygen isotopic abundances: Pure and Applied Chemistry, v. 66, no. 2, p. 273-276, http://dx.doi.org/10.1351/pac199466020273.

Coplen, T.B., Wildman, J.D., and Chen, Julie, 1991, Improvements in the gaseous hydrogen-water equilibration technique for hydrogen isotope-ratio analysis: Analytical Chemistry, v. 63, no. 9, p. 910-912, http://dx.doi.org/10.1021/ac00009a014.

Coplen, T.B., Hopple, J.A., Böhlke, J.K., Peiser, H.S., Rieder, S.E., Krouse, H.R., Rosman, K.J.R., Ding, T., Vocke, R.D., Jr., Revesz, K.M., Lamberty, A., Taylor, P., and DeBierve, P., 2002, Compilation of minimum and maximum isotope ratios of selected elements in naturally occurring terrestrial materials and reagents: U.S. Geological Survey Water-Resources Investigations Report 2001-4222, 98 p., https://pubs.er.usgs.gov/publication/wri014222.

Cunningham, W.L., and Schalk, C.W., 2011, Groundwater technical procedures of the U.S. Geological Survey: U.S. Geological Survey Techniques and Methods 1-A1, 154 p., accessed April 30, 2015, at http://pubs.usgs.gov/tm/1a1/.
Davis, T.A., Olsen, L.D., Fram, M.S., and Belitz, Kenneth, 2014, Updated study reporting levels (SRLs) for traceelement data collected for the California Groundwater Ambient Monitoring and Assessment (GAMA) Priority Basin Project, October 2009-March 2013: U.S. Geological Survey Scientific Investigations Report 2014-5105, 52 p., http://dx.doi.org/10.3133/sir20145105.

Donahue, D.J., Linick, T.W., and Jull, A.J.T., 1990, Isotoperatio and background corrections for accelerator mass spectrometry radiocarbon measurements: Radiocarbon, v. 32 , no. 2, p. 135-142.

Dwyer, G.S., and Vengosh, Avner, 2008, Alternative filament loading solution for accurate analysis of boron isotopes by negative thermal ionization mass spectrometry: Eos, Transactions, American Geophysical Union, v. 89, no. 53, abstract \#H51C-0824.

Eaton, G.F., Hudson, G.B., and Moran, J.E., 2004, Tritiumhelium-3 age-dating of groundwater in the Livermore Valley of California: American Chemical Society ACS Symposium Series, v. 868, p. 235-245, http://dx.doi.org/10.1021/bk-2004-0868.ch016.

Epstein, Samuel, and Mayeda, T.K., 1953, Variation of $\mathrm{O}^{18}$ content of water from natural sources: Geochimica et Cosmochimica Acta, v. 4, p. 213-224.

Faires, L.M., 1993, Methods of analysis by the U.S. Geological Survey National Water Quality Laboratory-Determination of metals in water by inductively coupled plasma-mass spectrometry: U.S. Geological Survey Open-File Report 92-634, 28 p., https://pubs.er.usgs.gov/publication/ofr92634.

Farrar, J.W., and Long, H.K., 1997, Report on the U.S. Geological Survey's evaluation program for standard reference samples distributed in September 1996-T-143 (trace constituents), T-145 (trace constituents), M-140 (major constituents), N-51 (nutrient constituents), N-52 (nutrient constituents), P-27 (low ionic strength constituents), and Hg-23 (mercury): U.S. Geological Survey Open-File Report 97-20, 145 p., https://pubs.er.usgs.gov/publication/ofr9720.

Fishman, M.J., ed., 1993, Methods of analysis by the U.S. Geological Survey National Water Quality Laboratory-Determination of inorganic and organic constituents in water and fluvial sediments: U.S. Geological Survey Open-File Report 93-125, 217 p., https://pubs.er.usgs.gov/publication/ofr93125.

Fishman, M.J., ed., and Friedman, L.C., 1989, Methods for determination of inorganic substances in water and fluvial sediments: U.S. Geological Survey Techniques of WaterResources Investigations, book 5, chap. A1, 545 p., https://pubs.er.usgs.gov/publication/twri05A1. 
Fram, M.S., Munday, Cathy, and Belitz, Kenneth, 2009, Groundwater quality data for the Tahoe-Martis study unit, 2007-Results from the California GAMA Program: U.S. Geological Survey Data Series 432, 88 p., at https://pubs.er.usgs.gov/publication/ds432.

Fram, M.S., Olsen, L.D., and Belitz, Kenneth, 2012, Evaluation of volatile organic compound (VOC) blank data and application of study reporting levels to groundwater data collected for the California GAMA Priority Basin Project, May 2004 through September 2010: U.S. Geological Survey Scientific Investigations Report 2012-5139, 94 p., https://pubs.er.usgs.gov/publication/sir20125139.

Gagnon, A.R., and Jones, G.A., 1993, AMS-graphite target production methods at the Woods Hole Oceanographic Institution during 1986-1991: Radiocarbon, v. 35, book 2, p. 301-310.

Garbarino, J.R., 1999, Methods of analysis by the U.S. Geological Survey National Water Quality Laboratory-Determination of dissolved arsenic, boron, lithium, selenium, strontium, thallium, and vanadium using inductively coupled plasma-mass spectrometry: U.S. Geological Survey Open-File Report 99-93, 31 p., https://pubs.er.usgs.gov/publication/ofr9993.

Garbarino, J.R., Kanagy, L.K., and Cree, M.E., 2006, Determination of elements in natural-water, biota, sediment, and soil samples using collision/reaction cell inductively coupled plasma-mass spectrometry: U.S. Geological Survey Techniques and Methods, book 5, chap. B1, 87 p., https://pubs.er.usgs.gov/publication/tm5B1.

Gran, Gunnar, 1952, Determination of the equivalence point in potentiometric titrations, Part II: Analyst, v. 77, p. 661-671, http://dx.doi.org/10.1039/AN9527700661.

Hahn, G.J., and Meeker W.Q., 1991, Statistical intervals-A guide for practitioners: New York, John Wiley \& Sons, $416 \mathrm{p}$.

Helsel, D.R., and Hirsch, R.M., 2002, Statistical methods in Water Resources: U.S. Geological Survey Techniques of Water-Resources Investigations 04-A3, 523 p., at https://pubs.er.usgs.gov/publication/twri04A3.

Hem, J.D., 1985, Study and interpretation of the chemical characteristics of natural water: U.S. Geological Survey Water Supply Paper 2254, 263 p., 3 pls., https://pubs.er.usgs.gov/publication/wsp2254.

Hoaglin, D.C., 1983, Letter values-A set of selected order statistics, in Hoaglin, D.C., Mosteller, F., and Tukey, J.W., eds., Understanding robust and exploratory data analysis: New York, John Wiley \& Sons, p. 33-54.
Koterba, M.T., Wilde, F.D., and Lapham, W.W., 1995, Groundwater data-collection protocols and procedures for the National Water-Quality Assessment Program-Collection and documentation of water-quality samples and related data: U.S. Geological Survey Open-File Report 95-399, 114 p., https://pubs.er.usgs.gov/publication/ofr95399.

Krieger, H.L., and Whittaker, E.L., 1980, Prescribed procedures for measurement of radioactivity in drinking water: U.S. Environmental Protection Agency EPA-600/4-80-032, 142 p., also available as PB80-224744 at http://www.ntis.gov.

Lane, S.L., Flanagan, Sarah, and Wilde, F.D., 2003, Selection of equipment for water sampling (ver. 2.0): U.S. Geological Survey Techniques of Water-Resources Investigations, book 9, chap. A2, accessed October 1, 2012, at http://pubs.water.usgs.gov/twri9A2/.

Lewis, M.E., 2006, Dissolved oxygen (ver. 2.1): U.S. Geological Survey Techniques of Water-Resources Investigations, book 9, chap. A6.2, accessed October 27, 2011, at http://pubs.water.usgs.gov/twri9A6.2/.

Maloney, T.J., ed., 2005, Quality management system, U.S. Geological Survey National Water Quality Laboratory: U.S. Geological Survey Open-File Report 2005-1263, accessed October 27, 2011, https://pubs.er.usgs.gov/publication/ofr20051263.

McCurdy, D.E., Garbarino, J.R., and Mullin, A.H., 2008, Interpreting and reporting radiological water-quality data: U.S. Geological Survey Techniques and Methods 5-B6, 33 p., https://pubs.er.usgs.gov/publication/tm5B6.

McLain, B.J., 1993, Methods of analysis by the U.S. Geological Survey National Water Quality Laboratory-Determination of chromium in water by graphite furnace atomic absorption spectrophotometry: U.S. Geological Survey Open-File Report 93-449, 16 p., https://pubs.er.usgs.gov/publication/ofr93449.

McNichol, A.P., Gagnon, A.R., and Osborne, E.A., 1992, Illumination of a black box-Analysis of gas composition during graphite target preparation: Radiocarbon, v. 34, book 3, p. 321-329.

McNichol, A.P., Jones, G.A., Hutton, D.L., Gagnon, A.R., and Key, R.M., 1994, The rapid preparation of seawater $\sum \mathrm{CO}_{2}$ for radiocarbon analysis at the National Ocean Sciences AMS Facility: Radiocarbon, v. 36, book 2, p. 237-246.

Moran, J.E., Hudson, G.B., Eaton, G.F., and Leif, Roald, 2002, A contamination vulnerability assessment for the Livermore-Amador and Niles Cone Groundwater Basins: Lawrence Livermore National Laboratory internal report UCRL-AR-148831, 25 p., http://www.swrcb.ca.gov/gama/docs/cas_llnl_alameda.pdf. 
Mueller, D.K., and Titus, C.J., 2005, Quality of nutrient data from streams and ground water sampled during water years 1992-2001: U.S. Geological Survey Scientific Investigations Report 2005-5106, 27 p., https://pubs.er.usgs.gov/publication/sir20055106.

Olsen, L.D., Fram, M.S., and Belitz, Kenneth, 2010, Review of trace-element field-blank data collected for the California Groundwater Ambient Monitoring and Assessment (GAMA) Program, May 2004January 2008: U.S. Geological Survey Scientific Investigations Report 2009-5220, 47 p., https://pubs.er.usgs.gov/publication/sir20095220.

Patton, C.J., and Kryskalla, J.R., 2003, Methods of analysis by the U.S. Geological Survey National Water Quality Laboratory-Evaluation of alkaline persulfate digestion as an alternative to Kjeldahl digestion for determination of total and dissolved nitrogen and phosphorus in water: U.S. Geological Survey WaterResources Investigations Report 2003-4174, 33 p., https://pubs.er.usgs.gov/publication/wri034174.

Pirkey, K.D., and Glodt, S.R., 1998, Quality control at the U.S. Geological Survey National Water Quality Laboratory: U.S. Geological Survey Fact Sheet 02698, 4 p., accessed October 27, 2011, at https://pubs.er.usgs.gov/publication/fs02698.

Radtke, D.B., Davis, J.V., and Wilde, F.D., 2005, Specific electrical conductance (ver. 1.2): U.S. Geological Survey Techniques of Water-Resources Investigations, book 9, chap. A6.3, accessed October 27, 2011, at http://pubs.water.usgs.gov/twri9A6.3/.

Révész, K., and Casciotti, K., 2007, Determination of the $\delta\left({ }^{15} \mathrm{~N} /{ }^{14} \mathrm{~N}\right)$ and $\delta\left({ }^{18} \mathrm{O} /{ }^{16} \mathrm{O}\right)$ of nitrate in water-RSIL Lab Code 2900, chap. C17, in Révész, Kinga, and Coplen, T.B., eds., Methods of the Reston Stable Isotope Laboratory: Reston, Virginia, U.S. Geological Survey Techniques and Methods, book 10, sec. C, chap. 17, 24 p.

Rose, D.L., Sandstrom, M.W., and Murtagh, L.K., 2016, Determination of heat purgeable and ambient purgeable volatile organic compounds in water by gas chromatography/mass spectrometry: U.S. Geological Survey Techniques and Methods 5-B12, $61 \mathrm{p}$., https://pubs.er.usgs.gov/publication/tm5B12.

Sandstrom, M.W., Stroppel, M.E., Foreman, W.T., and Schroeder, M.P., 2001, Methods of analysis by the U.S. Geological Survey National Water Quality Laboratory-Determination of moderateuse pesticides and selected degradates in water by C-18 solid-phase extraction and gas chromatography/ mass spectrometry: U.S. Geological Survey WaterResources Investigations Report 2001-4098, 70 p., https://pubs.er.usgs.gov/publication/wri20014098.
Sandstrom, M.W., Kanagy, L.K., Anderson, C.A., and Kanagy, C.J., 2015, Determination of pesticides and pesticide degradates in filtered water by direct aqueousinjection liquid chromatography-tandem mass spectrometry: U.S. Geological Survey Techniques and Methods 5-B11, 54 p., http://dx.doi.org/10.3133/tm5B11.

Schneider, R.J., Jones, G.A., McNichol, A.P., von Reden, K.F., Elder, K.L., Huang, Kelan, and Kessel, E.D., 1994, Methods for data screening, flagging and error analysis at the National Ocean Sciences AMS Facility in Nuclear Instruments and Methods in Physics Research Section B-Beam Interactions with Materials and Atoms: Woods Hole, Mass., National Ocean Sciences Accelerator Mass Spectrometry Facility, Woods Hole Oceanographic Institution, v. 92. no. 1-4, p. 172-175, http://dx.doi.org/10.1016/0168-583X(94)96000-3.

Shelton, J.L., Burow, K.R., Belitz, Kenneth, Dubrovsky, N.M., Land, Michael, and Gronberg, JoAnn, 2001, Low-level volatile organic compounds in active public supply wells as ground-water tracers in the Los Angeles physiographic basin, California, 2000: U.S. Geological Survey WaterResources Investigations Report 2001-4188, 35 p., https://pubs.er.usgs.gov/publication/wri20014188.

Stumm, Werner, and Morgan, J.J., 1996, Aquatic chemistryChemical equilibria and rates in natural waters ( $3 \mathrm{~d}$ ed.): New York, John Wiley and Sons, 1022 p.

Thatcher, L.L., Janzer, V.J., and Edwards, K.W., 1977, Methods for the determination of radioactive substances in water and fluvial sediments: U.S. Geological Survey Techniques of WaterResources Investigations 05-A5, 95 p., https://pubs.er.usgs.gov/publication/twri05A5.

Timme, P.J., 1995, National Water Quality Laboratory, 1995 services catalog: U.S. Geological Survey Open-File Report 95-352, 120 p., https://pubs.er.usgs.gov/publication/ofr95352.

U.S. Environmental Protection Agency, 2002, Guidelines for establishing procedures for the analysis of pollutants: U.S. Code of Federal Regulations, Title 40, 136 p.

U.S. Environmental Protection Agency, 2005, Method 331.0 - Determination of perchlorate in drinking water by liquid chromatography electrospray ionization mass spectrometry (Revision 1.0, January 2005): Office of Groundwater and Drinking Water, EPA Document \# 815-R05-007, 34 p., accessed January 28, 2015, at https://www.epa.gov/ground-water-and-drinking-water.

U.S. Geological Survey, [variously dated], National field manual for the collection of water-quality data: U.S. Geological Survey Techniques of Water-Resources Investigations, book 9, chap. A1-A9, accessed January 28, 2015, at http://water.usgs.gov/owq/FieldManual/. 
U.S. Geological Survey, 2006, National Elevation Dataset (NED): U.S. Geological Survey database, accessed January 28, 2015, at http://ned.usgs.gov/.

U.S. Geological Survey, 2009, Preferred method selection procedure, National Water Quality Laboratory website, accessed January 28, 2015, at http://wwwnwql.cr.usgs.gov/ dyn.shtml?Preferred_method_selection_procedure.

U.S. Geological Survey, 2015, Changes to National Water Quality Laboratory (NWQL) procedures used to establish and verify laboratory detection and reporting limits: U.S. Geological Survey National Water Quality Laboratory Technical Memorandum 15.02, 21 p., http://nwql.usgs.gov/ Public/tech_memos/nwql.2015-02.pdf.

Vengosh, Avner, Chivas, A.R., and McCulloch, M.T., 1989, Direct determination of boron and chlorine isotopic compositions in geological materials by negative thermalionization mass spectrometry: Chemical Geology-Isotope Geoscience section, v. 79, no. 4, p. 333-343, http://dx.doi.org/10.1016/0168-9622(89)90039-0.

Vogel, J.S., Nelson, D.E., and Southon, J.R., 1987, ${ }^{14} \mathrm{C}$ background levels in an accelerator mass spectrometry system: Radiocarbon, v. 29, no. 3, p. 323-333.

Weiss, R.F., 1968, Piggyback sampler for dissolved gas studies on sealed water samples: Deep Sea Research and Oceanographic Abstracts, v. 15, no. 6, p. 695-699, http://dx.doi.org/10.1016/0011-7471(68)90082-X.

Wilde, F.D., ed., 2004, Cleaning of equipment for water sampling (ver. 2.0): U.S. Geological Survey Techniques of Water-Resources Investigations, book 9, chap. A3, accessed January 28, 2015, at http:/pubs.water.usgs.gov/twri9A3/.
Wilde, F.D., 2006, Temperature (ver. 2.0): U.S. Geological Survey Techniques of Water-Resources Investigations, book 9, chap. A6.1, accessed January 28, 2015, at http://pubs.water.usgs.gov/twri9A6.1/.

Wilde, F.D., and Radtke, D.B., 2005, General information and guidelines (ver. 2.0): U.S. Geological Survey Techniques of Water-Resources Investigations, book 9, chap. A6.0, accessed January 28, 2015, at http://pubs.water.usgs.gov/twri9A6.0/.

Wilde, F.D., Radtke, D.B., Gibs, Jacob, and Iwatsubo, R.T., 1999, Collection of water samples: U.S. Geological Survey Techniques of Water-Resources Investigations, book 9, chap. A4, accessed January 28, 2015, at http://pubs.water.usgs.gov/twri9A4/.

Wilde, F.D., Radtke, D.B., Gibs, J., and Iwatsubo, R.T., 2004, Processing of water samples: U.S. Geological Survey Techniques of Water-Resources Investigations, book 9, chap. A5, accessed January 28, 2015, at http://pubs.water.usgs.gov/twri9A5/.

Wilde, F.D., Busenberg, Eurybiades, and Radtke, D.B., 2006, $\mathrm{pH}$ (ver. 2.0): U.S. Geological Survey Techniques of WaterResources Investigations, book 9, chap. A6.4, accessed January 28, 2015, at http://pubs.water.usgs.gov/twri9A6.4/.

Wright, M.T., Belitz, Kenneth, and Burton, C.A., 2005, California GAMA Program - Ground-water quality data in the San Diego drainages hydrogeologic province, California, 2004: U.S. Geological Survey Data Series 129, 102 p., at https://pubs.er.usgs.gov/publication/ds129. 
Table A-1. Analytical methods used for the determination of organic and inorganic constituents in water by the U.S. Geological Survey (USGS) National Water Quality Laboratory (NWOL) and contract laboratories.

[Laboratory entity codes in the USGS National Water Information System (NWIS) for laboratories other than the USGS National Water Quality Laboratory (NWQL) are given in parentheses after the laboratory names. Abbreviations: NRP, USGS National Research Program; EPA, U.S. Environmental Protection Agency; UV, ultraviolet; VOC, volatile organic compound]

\begin{tabular}{|c|c|c|c|}
\hline Analyte & Analytical method & Laboratory and analytical schedule & Citations \\
\hline \multicolumn{4}{|c|}{ Water-quality indicators } \\
\hline Field parameters & Calibrated field meters and test kits & USGS field measurement & U.S. Geological Survey, variously dated \\
\hline \multicolumn{4}{|c|}{ Organic constituents } \\
\hline VOCs & $\begin{array}{l}\text { Heat purgeable and ambient purgeable } \\
\text { gas chromatography/mass } \\
\text { spectrometry }\end{array}$ & NWQL, Schedules 4436 and 4437 & Rose and others, 2016 \\
\hline $\begin{array}{l}\text { Pesticides and pesticide } \\
\text { degradates }\end{array}$ & $\begin{array}{l}\text { Direct aqueous-injection liquid } \\
\text { chromatography-tandem mass } \\
\text { spectrometry }\end{array}$ & NWQL, Schedule 2437 & Sandstrom and others, 2015 \\
\hline \multicolumn{4}{|c|}{ Inorganic constituents } \\
\hline $\begin{array}{l}\text { Trace elements, and major and } \\
\text { minor ions }\end{array}$ & $\begin{array}{l}\text { Atomic absorption spectrometry, } \\
\text { colorimetry, ion-exchange } \\
\text { chromatography, inductively-coupled } \\
\text { plasma atomic emission spectrometry, } \\
\text { and mass spectrometry }\end{array}$ & $\begin{array}{l}\text { NWQL, Schedule 1948, and Schedules } 2710 \\
\text { and } 2750\end{array}$ & $\begin{array}{l}\text { Fishman and Friedman, 1989; Faires, 1993; } \\
\text { Fishman, 1993; McLain, 1993; American } \\
\text { Public Health Association, 1998; Garbarino, } \\
\text { 1999; Garbarino and others, } 2005\end{array}$ \\
\hline Nutrients & $\begin{array}{l}\text { Alkaline persulfate digestion, Kjedahl } \\
\text { digestion }\end{array}$ & NWQL, Schedule 2755 & Fishman, 1993; Patton and Kryskalla, 2003 \\
\hline Perchlorate & $\begin{array}{l}\text { Liquid chromatography with mass } \\
\text { spectrometry/mass spectrometry } \\
\text { (EPA Method 331.0) }\end{array}$ & $\begin{array}{l}\text { Weck Laboratories, Inc. [Weck], City of Industry, } \\
\text { California (CA-WECK), standard operating } \\
\text { procedure ORG099.R01 }\end{array}$ & U.S. Environmental Protection Agency, 2005 \\
\hline Radon-222 & Liquid scintillation counting & NWQL, Laboratory Code 1369 & American Society for Testing and Materials, 1998 \\
\hline $\begin{array}{l}\text { Gross alpha and gross beta } \\
\text { radioactivity ( } 72 \text {-hour and } \\
30 \text {-day) }\end{array}$ & $\begin{array}{l}\text { Alpha and beta activity counting } \\
\text { (EPA Method 900.0) }\end{array}$ & $\begin{array}{l}\text { TestAmerica Laboratory, Richland, Washington } \\
\text { (TestAmerica), NWQL Schedule } 1792\end{array}$ & Kreiger and Whittaker, 1980 \\
\hline \multicolumn{4}{|c|}{ Geochemical and age-dating tracers } \\
\hline Tritium & $\begin{array}{l}\text { Electrolytic enrichment-liquid } \\
\text { scintillation }\end{array}$ & $\begin{array}{l}\text { USGS Stable Isotope and Tritium Laboratory } \\
\text { (SITL), Menlo Park, California (USGSH3CA), } \\
\text { Laboratory Code } 1565\end{array}$ & Thatcher and others, 1977 \\
\hline $\begin{array}{l}\text { Stable isotopes of hydrogen and } \\
\text { oxygen in water }\end{array}$ & $\begin{array}{l}\text { Gaseous hydrogen and carbon dioxide- } \\
\text { water equilibration and stable-isotope } \\
\text { mass spectrometry }\end{array}$ & $\begin{array}{l}\text { USGS Stable Isotope Laboratory, Reston, Virginia } \\
\text { (USGSSIVA), NWQL Schedule } 1142\end{array}$ & $\begin{array}{l}\text { Epstein and Mayeda, 1953; Coplen and others, } \\
\text { 1991; Coplen, } 1994\end{array}$ \\
\hline $\begin{array}{l}\text { Stable isotopes of carbon in } \\
\text { dissolved inorganic carbon and } \\
\text { carbon-14 abundance }\end{array}$ & Accelerator mass spectrometry & $\begin{array}{l}\text { Woods Hole Oceanographic Institution, National } \\
\text { Ocean Sciences Accelerator Mass Spectrometry } \\
\text { Facility [NOSAMS], Woods Hole, Massachusetts } \\
\text { (MA-WHAMS), NWQL Schedule } 2255\end{array}$ & $\begin{array}{l}\text { Vogel and others, 1987; Donahue and others, } \\
\text { 1990; McNichol and others, 1992; Gagnon } \\
\text { and Jones, 1993; McNichol and others, 1994; } \\
\text { Schneider and others, } 1994\end{array}$ \\
\hline
\end{tabular}


Table A-1. Analytical methods used for the determination of organic and inorganic constituents in water by the U.S. Geological Survey (USGS) National Water Quality Laboratory (NWOL) and contract laboratories.-Continued

[Laboratory entity codes in the USGS National Water Information System (NWIS) for laboratories other than the USGS National Water Quality Laboratory (NWQL) are given in parentheses after the laboratory names. Abbreviations: NRP, USGS National Research Program; EPA, U.S. Environmental Protection Agency; UV, ultraviolet; VOC, volatile organic compound]

\begin{tabular}{|c|c|c|c|}
\hline Analyte & Analytical method & Laboratory and analytical schedule & Citations \\
\hline \multicolumn{4}{|c|}{ Geochemical and age-dating tracers-Continued } \\
\hline $\begin{array}{l}\text { Stable isotopes of nitrogen and } \\
\text { oxygen in dissolved nitrate }\end{array}$ & Denitrifier method and mass spectrometry & $\begin{array}{l}\text { USGS Stable Isotope Laboratory, Reston, Virginia } \\
\text { (USGSSIVA), NWQL Laboratory Code } 2900\end{array}$ & Révész and Casciotti, 2007 \\
\hline $\begin{array}{l}\text { Dissolved standard gases (argon, } \\
\text { carbon dioxide, methane, } \\
\text { nitrogen, and oxygen) }\end{array}$ & $\begin{array}{l}\text { Gas chromatography/thermal } \\
\text { conductivity detector and flame } \\
\text { ionization detector }\end{array}$ & $\begin{array}{l}\text { USGS Chlorofluorocarbon Laboratory, Reston, } \\
\text { Virginia (USGSCFCVA) }\end{array}$ & Busenberg and others, 2001 \\
\hline $\begin{array}{l}\text { Dissolved noble gases, and } \\
\text { helium isotope ratios }\end{array}$ & Mass spectrometry & $\begin{array}{l}\text { Lawrence Livermore National Laboratory (LLNL), } \\
\text { Livermore, California (CA-LLNL) }\end{array}$ & Moran and others, 2002; Eaton and others, 2004 \\
\hline \multicolumn{4}{|c|}{ Microbial indicators } \\
\hline $\begin{array}{l}\text { Escherichia coli (E.coli), total } \\
\text { coliform, and enterococci }\end{array}$ & $\begin{array}{l}\text { IDEXX Colilert }^{\circledR} \text { test kit (Standard } \\
\text { Method 9223) }\end{array}$ & USGS field presence/absence analysis & American Public Health Association, 1998 \\
\hline
\end{tabular}

Method 9223) 
Table A-2. Constituents detected in the blank water samples and the study reporting level (SRL) analysis used for the Madera/ Chowchilla-Kings Shallow Aquifer study unit, Groundwater Ambient Monitoring and Assessment (GAMA) Priority Basin Project, California, August 2013 to April 2014.

[LRL, laboratory reporting level; LT-MDL, long-term method detection level; mg/L, milligrams per liter; ng/L, nanograms per liter; nv, no value established; $\mu \mathrm{g} / \mathrm{L}$, micrograms per liter; $\mu \mathrm{S} / \mathrm{cm}$, microsiemens per centimeter; - , not detected]

\begin{tabular}{|c|c|c|c|c|c|c|c|}
\hline \multirow[b]{2}{*}{ Constituent } & \multirow[b]{2}{*}{ Units } & \multirow[b]{2}{*}{$\begin{array}{l}\text { LRL or } \\
\text { LT-MDL }\end{array}$} & \multirow{2}{*}{$\begin{array}{l}\text { Minimum } \\
\text { concentration } \\
\text { detected in } \\
\text { groundwater }\end{array}$} & \multicolumn{3}{|c|}{ Concentrations detected in blanks } & \multirow[b]{2}{*}{ SRL } \\
\hline & & & & $\begin{array}{c}\text { Grundfos } \\
\text { pump blank } \\
(\mathrm{n}=1)\end{array}$ & $\begin{array}{l}\text { Field blanks } \\
\qquad(n=7)\end{array}$ & $\begin{array}{c}\text { Source-solution } \\
\text { blanks } \\
(n=3)\end{array}$ & \\
\hline \multicolumn{8}{|c|}{ Volatile organic compounds } \\
\hline Cyclohexanone & $\mu \mathrm{g} / \mathrm{L}$ & 1.2 & 0.46 & 0.75 & - & - & $\mathrm{nv}$ \\
\hline 1,1-Difluoroethane & $\mu \mathrm{g} / \mathrm{L}$ & 0.022 & - & - & 0.014 & - & nv \\
\hline Isopropyl alcohol & $\mu \mathrm{g} / \mathrm{L}$ & 0.8 & 0.44 & - & 0.66 & - & all data \\
\hline tert-Butyl alcohol & $\mu \mathrm{g} / \mathrm{L}$ & 0.24 & 0.18 & - & 0.14 & - & nv \\
\hline \multicolumn{8}{|c|}{ Pesticides and degradates } \\
\hline 2-Amino- $N$-isopropylbenzamide & $\mathrm{ng} / \mathrm{L}$ & 4 & - & - & 0.7 & - & nv \\
\hline$N$-(3,4-Dichlorophenyl)- $N$ '-methylurea & $\mathrm{ng} / \mathrm{L}$ & 5 & - & - & 0.8 & - & nv \\
\hline Piperonyl butoxide & $\mathrm{ng} / \mathrm{L}$ & 4 & - & - & 0.3 & - & nv \\
\hline Simazine & $\mathrm{ng} / \mathrm{L}$ & 10 & 8.7 & - & 7.5 & - & nv \\
\hline \multicolumn{8}{|c|}{ Trace elements } \\
\hline Chromium & $\mu \mathrm{g} / \mathrm{L}$ & 0.07 & 0.32 & 0.12 & - & - & $\mathrm{nv}$ \\
\hline Cobalt & $\mu \mathrm{g} / \mathrm{L}$ & 0.023 & na & - & $\begin{array}{c}0.14,0.17,0.13 \\
0.06,0.15,0.09\end{array}$ & - & all data \\
\hline Copper & $\mu \mathrm{g} / \mathrm{L}$ & 0.8 & 0.8 & - & 1 & - & 2.1 \\
\hline Lead & $\mu \mathrm{g} / \mathrm{L}$ & 0.025 & 0.043 & 0.04 & - & - & 0.82 \\
\hline Manganese & $\mu \mathrm{g} / \mathrm{L}$ & 0.15 & 0.43 & 0.83 & 0.28 & - & 0.66 \\
\hline Molybdenum & $\mu \mathrm{g} / \mathrm{L}$ & 0.014 & 0.07 & 0.02 & - & - & $\mathrm{nv}$ \\
\hline Nickel & $\mu \mathrm{g} / \mathrm{L}$ & 0.09 & 0.2 & 0.17 & 0.74 & - & 0.21 \\
\hline Zinc & $\mu \mathrm{g} / \mathrm{L}$ & 1.4 & 2.1 & 1.9 & 2.1 & - & 6.2 \\
\hline \multicolumn{8}{|c|}{ Other inorganic constituents } \\
\hline Silica & $\mathrm{mg} / \mathrm{L}$ & 0.018 & 16.7 & - & 0.05 & - & nv \\
\hline Ammonia, as nitrogen & $\mathrm{mg} / \mathrm{L}$ & 0.010 & 0.019 & - & $0.011,0.011,0.010$ & - & $\mathrm{nv}$ \\
\hline
\end{tabular}


Table A-3A. Quality-control summary for replicate pair analyses of organic and inorganic constituents in groundwater samples collected from the Madera/Chowchilla-Kings Shallow Aquifer study unit, Groundwater Ambient Monitoring and Assessment (GAMA) Priority Basin Project, California, August 2013 to April 2014.

[Constituents for which all replicate pairs were non-detections are not listed. Replicate pairs were evaluated using standard deviation if the average concentration for the pair was less than or equal to five times the LRL, and with relative standard deviation if the average concentration was greater than five times the LRL. Acceptable replicates are defined as those having relative standard deviation less than 10 percent or standard deviation less than half of the LRL (see appendix). Abbreviations: $\mathrm{CaCO}_{3}$, calcium carbonate; E, estimated or having a higher degree of uncertainty; LRL, laboratory reporting level; mg/L, milligrams per liter; ng/L, nanograms per liter; nv, no value in category; <, less than; $\mu \mathrm{g} / \mathrm{L}$, micrograms per liter; $\mu \mathrm{S} / \mathrm{cm}$, microsiemens per centimeter]

\begin{tabular}{|c|c|c|c|c|c|c|}
\hline \multirow[b]{2}{*}{ Constituent } & \multirow{2}{*}{$\begin{array}{c}\text { Number } \\
\text { of pairs of } \\
\text { nondetections / } \\
\text { total number of } \\
\text { replicate pairs }{ }^{1}\end{array}$} & \multirow[b]{2}{*}{ LRL } & \multicolumn{2}{|c|}{ Replicate pairs evaluated with standard deviation } & \multicolumn{2}{|c|}{ Replicate pairs evaluated with relative standard deviation } \\
\hline & & & $\begin{array}{l}\text { Concentrations } \\
\text { for } \\
\text { acceptable } \\
\text { pairs }\end{array}$ & $\begin{array}{c}\text { Concentrations } \\
\text { for } \\
\text { unacceptable } \\
\text { pairs }\end{array}$ & $\begin{array}{l}\text { Concentrations } \\
\text { for } \\
\text { acceptable } \\
\text { pairs }\end{array}$ & $\begin{array}{c}\text { Concentrations } \\
\text { for } \\
\text { unacceptable } \\
\text { pairs }\end{array}$ \\
\hline \multicolumn{7}{|c|}{ Volatile organic compounds ( $\mu \mathrm{g} / \mathrm{L}$ ) } \\
\hline 1,2,3-Trichloropropane & $6 / 7$ & 0.006 & nv & nv & $(0.071,0.069)$ & nv \\
\hline 1,2-Dichloropropane & $5 / 7$ & 0.004 & $(0.006,0.006)$ & nv & $(0.081,0.081)$ & nv \\
\hline 1,2-Dibromo-3-chloropropane & $6 / 7$ & 0.02 & nv & nv & $(0.172,0.165)$ & nv \\
\hline Carbon disulfide & $6 / 7$ & 0.1 & nv & $(<0.10,0.17)$ & nv & nv \\
\hline Isopropyl alcohol & $6 / 7$ & 0.8 & nv & $(<0.8,1.3)$ & nv & nv \\
\hline \multicolumn{7}{|c|}{ Pesticides and degradates (ng/L) } \\
\hline $\begin{array}{l}\text { 2-Chloro-4,6-diamino-s-triazine } \\
\text { (CAAT) }\end{array}$ & $1 / 7$ & 50 & $(27,20) ;(95,88) ;(101,86)$ & $(107,<65)$ & $(208,205) ;(175,186)$ & $\mathrm{nv}$ \\
\hline $\begin{array}{l}\text { 2-Chloro-4-isopropylamino- } \\
\text { 6-amino-s-triazine (CIAT, } \\
\text { deethylatrazine) }\end{array}$ & $6 / 7$ & 11 & $(\mathrm{E} 10,<11)$ & nv & nv & $\mathrm{nv}$ \\
\hline $\begin{array}{l}\text { 2-Chloro-6-ethylamino-4-amino- } \\
\text { s-triazine }\end{array}$ & $5 / 7$ & 50 & $(30,35) ;(61,42)$ & nv & nv & nv \\
\hline $\begin{array}{l}\text { 2-Hydroxy-4-isopropylamino- } \\
\text { 6-ethylamino-s-triazine }\end{array}$ & $6 / 7$ & 8 & $(5,5)$ & nv & nv & nv \\
\hline Demethyl hexazinone B & $6 / 7$ & 3 & $(8,8)$ & nv & nv & $\mathrm{nv}$ \\
\hline Demethyl norflurazon & $6 / 7$ & 4 & nv & nv & $(91,97)$ & nv \\
\hline Hexazinone & $6 / 7$ & 3 & $(2,3)$ & nv & nv & $\mathrm{nv}$ \\
\hline Metolachlor sulfonic acid & $6 / 7$ & 68 & $(97,107)$ & nv & nv & nv \\
\hline Norflurazon & $6 / 7$ & 5 & $(18,17)$ & nv & nv & nv \\
\hline \multicolumn{7}{|c|}{ Major and minor ions (mg/L) } \\
\hline Bromide & $2 / 7$ & 0.01 & $(0.04,0.04)$ & nv & $\begin{array}{l}(0.09,0.09) ;(0.12,0.12) \\
(0.14,0.15) ;(1.11,1.15)\end{array}$ & nv \\
\hline Calcium & $0 / 7$ & 0.022 & $\mathrm{nv}$ & nv & $\begin{array}{c}(49,49.4) ;(49.2,48.4) ;(52.7,51.9) \\
(70.9,72.6) ;(126.8,127) \\
\quad(14.6,14.4) ;(28.4,28.2)\end{array}$ & nv \\
\hline
\end{tabular}


Table A-3A. Quality-control summary for replicate pair analyses of organic and inorganic constituents in groundwater samples collected from the Madera/Chowchilla-Kings Shallow Aquifer study unit, Groundwater Ambient Monitoring and Assessment (GAMA) Priority Basin Project, California, August 2013 to April 2014.—Continued

[Constituents for which all replicate pairs were non-detections are not listed. Replicate pairs were evaluated using standard deviation if the average concentration for the pair was less than or equal to five times the LRL, and with relative standard deviation if the average concentration was greater than five times the LRL. Acceptable replicates are defined as those having relative standard deviation less than 10 percent or standard deviation less than half of the LRL (see appendix). Abbreviations: $\mathrm{CaCO}_{3}$, calcium carbonate; E, estimated or having a higher degree of uncertainty; LRL, laboratory reporting level; mg/L, milligrams per liter; $\mathrm{ng} / \mathrm{L}$, nanograms per liter; nv, no value in category; $<$, less than; $\mu \mathrm{g} / \mathrm{L}$, micrograms per liter; $\mu \mathrm{S} / \mathrm{cm}$, microsiemens per centimeter]

\begin{tabular}{|c|c|c|c|c|c|c|}
\hline \multirow[b]{2}{*}{ Constituent } & \multirow{2}{*}{$\begin{array}{c}\text { Number } \\
\text { of pairs of } \\
\text { nondetections / } \\
\text { total number of } \\
\text { replicate pairs }{ }^{1}\end{array}$} & \multirow[b]{2}{*}{ LRL } & \multicolumn{2}{|c|}{ Replicate pairs evaluated with standard deviation } & \multicolumn{2}{|c|}{ Replicate pairs evaluated with relative standard deviation } \\
\hline & & & $\begin{array}{l}\text { Concentrations } \\
\text { for } \\
\text { acceptable } \\
\text { pairs }\end{array}$ & $\begin{array}{l}\text { Concentrations } \\
\text { for } \\
\text { unacceptable } \\
\text { pairs }\end{array}$ & $\begin{array}{l}\text { Concentrations } \\
\text { for } \\
\text { acceptable } \\
\text { pairs }\end{array}$ & $\begin{array}{c}\text { Concentrations } \\
\text { for } \\
\text { unacceptable } \\
\text { pairs }\end{array}$ \\
\hline \multicolumn{7}{|c|}{ Major and minor ions (mg/L)_Continued } \\
\hline Chloride & $0 / 7$ & 0.06 & nv & nv & $\begin{array}{c}(22.3,22.4) ;(8.3,8.2) ;(2.1,2.1) \\
\quad(27.4,27.4) ;(41.8,41.9) \\
\quad(2.8,2.8) ;(280.3,268.4)\end{array}$ & nv \\
\hline Fluoride & $0 / 7$ & 0.04 & $\begin{array}{l}(0.09,0.1) ;(0.12,0.12) ;(0.02,0.02) \\
(0.05,0.05) ;(0.1,0.1) ;(0.08,0.08)\end{array}$ & $\mathrm{nv}$ & $(0.4,0.38)$ & $\mathrm{nv}$ \\
\hline Iodide & $1 / 7$ & 0.001 & $(0.002,0.001) ;(0.001,0.001)$ & nv & $\begin{array}{l}(0.008,0.008) ;(0.011,0.011) \\
(0.007,0.006) ;(0.392,0.379)\end{array}$ & nv \\
\hline Magnesium & $0 / 7$ & 0.011 & nv & nv & $\begin{array}{c}(9.3,9.3) ;(20.7,20.7) ;(18.3,18.2) \\
(19.6,19.6) ;(35.4,35.6) \\
(3.9,3.8) ;(4.7,4.6)\end{array}$ & nv \\
\hline Potassium & $0 / 7$ & 0.03 & nv & nv & $\begin{array}{l}(3.2,3.2) ;(3.4,3.5) ;(2.8,2.8) ;(5,5) \\
\quad(3.7,3.8) ;(1.7,1.7) ;(2.7,2.6)\end{array}$ & nv \\
\hline Silica & $0 / 7$ & 0.018 & nv & nv & $\begin{array}{c}(67.7,68.4) ;(66.6,65.1) ;(37,36.8) \\
\quad(32,32.4) ;(58.3,58.4) \\
\quad(34.8,34.7) ;(28.6,28.4)\end{array}$ & nv \\
\hline Sodium & $0 / 7$ & 0.06 & nv & nv & $\begin{array}{c}(14.1,14.2) ;(44.2,43.5) ;(15.6,15.7) \\
(35.4,33.7) ;(50.3,50.6) \\
\quad(7.5,7.7) ;(289.8,288.8)\end{array}$ & nv \\
\hline Sulfate & $0 / 7$ & 0.09 & nv & nv & $\begin{array}{l}(6.6,6.6) ;(51.3,51.3) ;(8.1,8.1) ;(51.9,52) \\
\quad(163.5,163.7) ;(7.7,7.7) ;(106.7,101.6)\end{array}$ & nv \\
\hline Total dissolved solids & $0 / 7$ & 20 & nv & nv & $\begin{array}{c}(271,267) ;(381,381) ;(265,267) \\
(404,409) ;(742,737) ;(113,111) ;(897,910)\end{array}$ & nv \\
\hline \multicolumn{7}{|c|}{ Nutrients (mg/L) } \\
\hline Ammonium-N & $6 / 7$ & 0.01 & $(0.021,0.023)$ & nv & nv & nv \\
\hline Nitrate-N & $2 / 7$ & 0.04 & nv & nv & $\begin{array}{l}(3.4,3.4) ;(3.5,3.6) ;(8.5,8.5) \\
\quad(24.1,23.8) ;(0.7,0.8)\end{array}$ & nv \\
\hline Nitrite-N & $4 / 7$ & 0.001 & $(0.002,0.002)$ & $(<0.001,0.002)$ & $(0.007,0.007)$ & nv \\
\hline Orthophosphate-P & $0 / 7$ & 0.004 & $(0.019,0.02)$ & nv & $\begin{array}{l}(0.034,0.033) ;(0.04,0.04) ;(0.023,0.023) \\
(0.022,0.022) ;(0.033,0.033) ;(0.078,0.081)\end{array}$ & nv \\
\hline
\end{tabular}


Table A-3A. Quality-control summary for replicate pair analyses of organic and inorganic constituents in groundwater samples collected from the Madera/Chowchilla-Kings Shallow Aquifer study unit, Groundwater Ambient Monitoring and Assessment (GAMA) Priority Basin Project, California, August 2013 to April 2014.-Continued

[Constituents for which all replicate pairs were non-detections are not listed. Replicate pairs were evaluated using standard deviation if the average concentration for the pair was less than or equal to five times the LRL, and with relative standard deviation if the average concentration was greater than five times the LRL. Acceptable replicates are defined as those having relative standard deviation less than 10 percent or standard deviation less than half of the LRL (see appendix). Abbreviations: $\mathrm{CaCO}_{3}$, calcium carbonate; E, estimated or having a higher degree of uncertainty; LRL, laboratory reporting level; mg/L, milligrams per liter; ng/L, nanograms per liter; nv, no value in category; <, less than; $\mu \mathrm{g} / \mathrm{L}$, micrograms per liter; $\mu \mathrm{S} / \mathrm{cm}$, microsiemens per centimeter]

\begin{tabular}{|c|c|c|c|c|c|c|}
\hline \multirow[b]{2}{*}{ Constituent } & \multirow{2}{*}{$\begin{array}{c}\text { Number } \\
\text { of pairs of } \\
\text { nondetections / } \\
\text { total number of } \\
\text { replicate pairs }{ }^{1}\end{array}$} & \multirow[b]{2}{*}{ LRL } & \multicolumn{2}{|c|}{ Replicate pairs evaluated with standard deviation } & \multicolumn{2}{|c|}{ Replicate pairs evaluated with relative standard deviation } \\
\hline & & & $\begin{array}{c}\text { Concentrations } \\
\text { for } \\
\text { acceptable } \\
\text { pairs }\end{array}$ & $\begin{array}{c}\text { Concentrations } \\
\text { for } \\
\text { unacceptable } \\
\text { pairs }\end{array}$ & $\begin{array}{c}\text { Concentrations } \\
\text { for } \\
\text { acceptable } \\
\text { pairs }\end{array}$ & $\begin{array}{c}\text { Concentrations } \\
\text { for } \\
\text { unacceptable } \\
\text { pairs }\end{array}$ \\
\hline \multicolumn{7}{|c|}{ Nutrients (mg/L)—Continued } \\
\hline Total nitrogen & $1 / 7$ & 0.05 & $(0.1,0.1)$ & nv & $\begin{array}{l}(3.4,3.3) ;(3.7,3.7) ;(8.4,8.2) \\
\quad(23.5,21.8) ;(0.8,0.8)\end{array}$ & nv \\
\hline \multicolumn{7}{|c|}{ Trace elements $(\mu \mathrm{g} / \mathrm{L})$} \\
\hline Aluminum & $0 / 7$ & 2.2 & $\begin{array}{l}(4.3,4.4) ;(4.2,4.7) ;(3.4,2.5) ;(2.8 \\
2.6) ;(3.1,3.4) ;(2.8,2.5) ;(2.4,2.4)\end{array}$ & nv & nv & nv \\
\hline Antimony & $1 / 7$ & 0.027 & $\begin{array}{l}(0.08,0.08) ;(0.1,0.11) ;(0.04,0.04) \\
(0.05,0.05) ;(0.04,0.04) ;(0.03,0.03)\end{array}$ & nv & nv & nv \\
\hline Arsenic & $0 / 7$ & 0.04 & nv & nv & $\begin{array}{c}(3.9,3.9) ;(4,4.1) ;(0.2,0.2) ;(0.8,0.8) \\
(2.5,2.6) ;(1.5,1.5) ;(9,9)\end{array}$ & nv \\
\hline Barium & $0 / 7$ & 0.1 & $\mathrm{nv}$ & nv & $\begin{array}{c}(164,162) ;(20,20) ;(29,28) ;(162,162) \\
(120,122) ;(11,11) ;(78,78)\end{array}$ & $\mathrm{nv}$ \\
\hline Beryllium & $6 / 7$ & 0.006 & $\mathrm{nv}$ & nv & $(0.09,0.09)$ & nv \\
\hline Boron & $0 / 7$ & 3 & $(13,14) ;(13,13) ;(13,13)$ & $\mathrm{nv}$ & $(20,20) ;(75,75) ;(26,26) ;(1,459,1,518)$ & nv \\
\hline Cadmium & $5 / 7$ & 0.016 & $(0.03,0.04)$ & $(0.04,<0.016)$ & $\mathrm{nv}$ & nv \\
\hline Chromium & $2 / 7$ & 0.07 & $\mathrm{nv}$ & nv & $\begin{array}{l}(0.9,0.9) ;(0.6,0.7) ;(1.4,1.4) \\
(1.6,1.6) ;(0.5,0.5)\end{array}$ & nv \\
\hline Copper & $5 / 7$ & 2.1 & $(3.9,4.2)$ & $(<2.1,2.6)$ & nv & nv \\
\hline Iron & $4 / 7$ & 6 & $(9,10)$ & nv & $(221,217) ;(66,66)$ & nv \\
\hline Lead & $6 / 7$ & 0.82 & $(1.7,1.7)$ & nv & $\mathrm{nv}$ & nv \\
\hline Lithium & $0 / 7$ & 0.22 & $(0.5,0.5)$ & nv & $\begin{array}{l}(4.2,4.2) ;(24.1,24.3) ;(1.8,1.9) \\
\quad(3.3,3.3) ;(1.2,1.2) ;(7.8,7.7)\end{array}$ & nv \\
\hline Manganese & $5 / 7$ & 0.66 & $\mathrm{nv}$ & $\mathrm{nv}$ & $(9,10) ;(414,410)$ & nv \\
\hline Molybdenum & $0 / 7$ & 0.014 & $\mathrm{nv}$ & nv & $\begin{array}{c}(1.3,1.2) ;(11,11.1) ;(0.1,0.1) ;(0.9,0.9) \\
(1.3,1.3) ;(0.7,0.7) ;(7.6,7.4)\end{array}$ & $\mathrm{nv}$ \\
\hline Nickel & $1 / 7$ & 0.21 & $\begin{array}{c}(0.7,0.7) ;(0.8,0.9) ;(1,1.1) ;(0.6 \\
0.6) ;(0.8,0.9)\end{array}$ & $(<0.21,0.4)$ & nv & nv \\
\hline
\end{tabular}


Table A-3A. Quality-control summary for replicate pair analyses of organic and inorganic constituents in groundwater samples collected from the Madera/Chowchilla-Kings Shallow Aquifer study unit, Groundwater Ambient Monitoring and Assessment (GAMA) Priority Basin Project, California, August 2013 to April 2014.—Continued

[Constituents for which all replicate pairs were non-detections are not listed. Replicate pairs were evaluated using standard deviation if the average concentration for the pair was less than or equal to five times the LRL, and with relative standard deviation if the average concentration was greater than five times the LRL. Acceptable replicates are defined as those having relative standard deviation less than 10 percent or standard deviation less than half of the LRL (see appendix). Abbreviations: $\mathrm{CaCO}_{3}$, calcium carbonate; E, estimated or having a higher degree of uncertainty; LRL, laboratory reporting level; $\mathrm{mg} / \mathrm{L}$, milligrams per liter; $\mathrm{ng} / \mathrm{L}$, nanograms per liter; nv, no value in category; $<$, less than; $\mu \mathrm{g} / \mathrm{L}$, micrograms per liter; $\mu \mathrm{S} / \mathrm{cm}$, microsiemens per centimeter]

\begin{tabular}{|c|c|c|c|c|c|c|}
\hline \multirow[b]{2}{*}{ Constituent } & \multirow{2}{*}{$\begin{array}{c}\text { Number } \\
\text { of pairs of } \\
\text { nondetections / } \\
\text { total number of } \\
\text { replicate pairs }{ }^{1}\end{array}$} & \multirow[b]{2}{*}{ LRL } & \multicolumn{2}{|c|}{ Replicate pairs evaluated with standard deviation } & \multicolumn{2}{|c|}{ Replicate pairs evaluated with relative standard deviation } \\
\hline & & & $\begin{array}{l}\text { Concentrations } \\
\text { for } \\
\text { acceptable } \\
\text { pairs }\end{array}$ & $\begin{array}{l}\text { Concentrations } \\
\text { for } \\
\text { unacceptable } \\
\text { pairs }\end{array}$ & $\begin{array}{l}\text { Concentrations } \\
\text { for } \\
\text { acceptable } \\
\text { pairs }\end{array}$ & $\begin{array}{c}\text { Concentrations } \\
\text { for } \\
\text { unacceptable } \\
\text { pairs }\end{array}$ \\
\hline \multicolumn{7}{|c|}{ Trace elements $(\mu \mathrm{g} / \mathrm{L})$-Continued } \\
\hline Selenium & $1 / 7$ & 0.03 & $(0.08,0.09) ;(0.06,0.06)$ & nv & $\begin{array}{l}(0.87,0.87) ;(0.2,0.24) \\
(0.41,0.42) ;(0.21,0.21)\end{array}$ & nv \\
\hline Strontium & $0 / 7$ & 0.2 & $\mathrm{nv}$ & nv & $\begin{array}{c}(368,367) ;(412,417) ;(406,412) \\
(588,597) ;(647,656) ;(112,111) ;(315,314)\end{array}$ & nv \\
\hline Uranium & $0 / 7$ & 0.004 & $\mathrm{nv}$ & nv & $\begin{array}{l}(3.7,3.6) ;(61.4,62.4) ;(7.8,7.9) \\
\quad(20.5,20.4) ;(11.7,11.7) \\
\quad(2.2,2.2) ;(10.1,10.6)\end{array}$ & $\mathrm{nv}$ \\
\hline Vanadium & $0 / 7$ & 0.08 & $\mathrm{nv}$ & nv & $\begin{array}{c}(21.7,21.6) ;(16.3,16.7) ;(1.4,1.4) ; \\
(7.7,7.7) ;(37.2,37.6) ;(8.3,8.3) ;(3,2.9)\end{array}$ & nv \\
\hline Zinc & $3 / 7$ & 6.2 & $\begin{array}{l}(26.6,26.9) ;(18.7,21.1) \\
(13.7,13.5) ;(27.5,29.4)\end{array}$ & $\mathrm{nv}$ & $\mathrm{nv}$ & $\mathrm{nv}$ \\
\hline \multicolumn{7}{|c|}{ Other inorganic constituents (units as indicated) } \\
\hline $\begin{array}{l}\text { Alkalinity (field; } \mathrm{mg} / \mathrm{L} \text { as } \\
\qquad \mathrm{CaCO}_{3} \text { ) }\end{array}$ & $0 / 3$ & $\mathrm{nv}$ & $\mathrm{nv}$ & nv & $(130,127) ;(217,222) ;(228,259)$ & nv \\
\hline Alkalinity (lab; mg/L as $\mathrm{CaCO}_{3}$ ) & $0 / 7$ & 4.6 & $\mathrm{nv}$ & nv & $\begin{array}{c}(144,144) ;(228,228) ;(241,241) ; \\
(224,224) ;(257,257) ;(56,56) ;(242,241)\end{array}$ & nv \\
\hline pH (lab; standard units) & $0 / 7$ & $\mathrm{nv}$ & $\mathrm{nv}$ & $\mathrm{nv}$ & $\begin{array}{c}(7.8,7.8) ;(7.8,7.8) ;(7.1,7.2) ;(7.9,7.9) \\
\quad(7.6,7.6) ;(7.9,7.9) ;(8,8.1)\end{array}$ & $\mathrm{nv}$ \\
\hline $\begin{array}{l}\text { Specific conductance }(\mathrm{lab} ; \mu \mathrm{S} / \\
\mathrm{cm})\end{array}$ & $0 / 7$ & 5 & $\mathrm{nv}$ & $\mathrm{nv}$ & $\begin{array}{l}(370,371) ;(546,544) ;(428,431) \\
\quad(631,633) ;(1,015,1,020) \\
\quad(137,137) ;(1,523,1,516)\end{array}$ & $\mathrm{nv}$ \\
\hline Perchlorate & $2 / 7$ & 0.1 & $(0.28,0.28) ;(0.14,0.11) ;(0.19,0.18)$ & nv & $(2.76,2.73) ;(1.21,1.07)$ & nv \\
\hline$\delta^{2} \mathrm{H}$ of water & $0 / 7$ & $\mathrm{nv}$ & $\mathrm{nv}$ & nv & $\begin{array}{c}(-87.8,-88.6) ;(-94.9,-95.2) \\
(-96.1,-96.5) ;(-86.9,-86.0) ; \\
(-74.6,-73.1) ;(-99.5,-99.6) ;(-70.2,-71.2)\end{array}$ & nv \\
\hline
\end{tabular}


Table A-3A. Quality-control summary for replicate pair analyses of organic and inorganic constituents in groundwater samples collected from the Madera/Chowchilla-Kings Shallow Aquifer study unit, Groundwater Ambient Monitoring and Assessment (GAMA) Priority Basin Project, California, August 2013 to April 2014.-Continued

[Constituents for which all replicate pairs were non-detections are not listed. Replicate pairs were evaluated using standard deviation if the average concentration for the pair was less than or equal to five times the LRL, and with relative standard deviation if the average concentration was greater than five times the LRL. Acceptable replicates are defined as those having relative standard deviation less than 10 percent or standard deviation less than half of the LRL (see appendix). Abbreviations: $\mathrm{CaCO}_{3}$, calcium carbonate; E, estimated or having a higher degree of uncertainty; LRL, laboratory reporting level; $\mathrm{mg} / \mathrm{L}$, milligrams per liter; $\mathrm{ng} / \mathrm{L}$, nanograms per liter; nv, no value in category; <, less than; $\mu \mathrm{g} / \mathrm{L}$, micrograms per liter; $\mu \mathrm{S} / \mathrm{cm}$, microsiemens per centimeter]

\begin{tabular}{|c|c|c|c|c|c|c|}
\hline \multirow[b]{2}{*}{ Constituent } & \multirow{2}{*}{$\begin{array}{c}\text { Number } \\
\text { of pairs of } \\
\text { nondetections / } \\
\text { total number of } \\
\text { replicate pairs }{ }^{1}\end{array}$} & \multirow[b]{2}{*}{ LRL } & \multicolumn{2}{|c|}{ Replicate pairs evaluated with standard deviation } & \multicolumn{2}{|c|}{ Replicate pairs evaluated with relative standard deviation } \\
\hline & & & $\begin{array}{l}\text { Concentrations } \\
\text { for } \\
\text { acceptable } \\
\text { pairs }\end{array}$ & $\begin{array}{l}\text { Concentrations } \\
\text { for } \\
\text { unacceptable } \\
\text { pairs }\end{array}$ & $\begin{array}{l}\text { Concentrations } \\
\text { for } \\
\text { acceptable } \\
\text { pairs }\end{array}$ & $\begin{array}{c}\text { Concentrations } \\
\text { for } \\
\text { unacceptable } \\
\text { pairs }\end{array}$ \\
\hline \multicolumn{7}{|c|}{ Other inorganic constituents (units as indicated) —Continued } \\
\hline$\delta^{18} \mathrm{O}$ of water & $0 / 7$ & nv & nv & nv & $\begin{array}{c}(-11.83,-12.01) ;(-12.83,-12.78) ; \\
(-12.98,-12.98) ;(-11.52,-11.63) ; \\
(-9.63,-9.66) ;(-13.53,-13.53) ; \\
(-9.36,-9.36)\end{array}$ & nv \\
\hline$\delta^{15} \mathrm{~N}$ of nitrate & $0 / 5$ & nv & nv & nv & $\begin{array}{c}(5.54,5.66) ;(10.49,10.63) ;(4.14,4.10) \\
(7.18,7.18) ;(4.05,4.04)\end{array}$ & nv \\
\hline$\delta^{18} \mathrm{O}$ of nitrate & $0 / 5$ & nv & nv & nv & $\begin{array}{l}(3.50,3.45) ;(2.39,2.58) \\
(1.69,1.60) ;(-0.92,-1.14)\end{array}$ & $(0.05,0.20)$ \\
\hline $\begin{array}{l}\delta^{13} \mathrm{C} \text { of dissolved inorganic } \\
\text { carbon }\end{array}$ & $0 / 7$ & nv & nv & nv & $\begin{array}{c}(-14.76,-14.78) ;(-16.80,-16.80) ; \\
(-13.25,-13.26) ;(-15.58,-15.54) ; \\
(-14.27,-14.27) ;(-15.96,-16.11) ; \\
(-28.66,-28.67)\end{array}$ & nv \\
\hline
\end{tabular}

etections. The IT-MDL is equal to half of the LRL. 
Table A-3B. Quality-control summary for replicate pair analyses of radioactive constituents in groundwater samples collected from the Madera/Chowchilla-Kings Shallow Aquifer study unit, Groundwater Ambient Monitoring and Assessment (GAMA) Priority Basin Project, California, August 2013 to April 2014.

[Seven replicate pairs were analyzed for each constituent. Constituents for which all replicate pairs were non-detections are not listed. Replicate pairs were evaluated using standard deviation if the average concentration for the pair was less than or equal to five times the LRL, and with relative standard deviation if the average concentration was greater than five times the LRL. Acceptable replicates are defined as those having relative standard deviation less than 10 percent or standard deviation less than half of the LRL (see appendix). Abbreviations: LRL, laboratory reporting level; nv, no value in category; \pm , plus or minus]

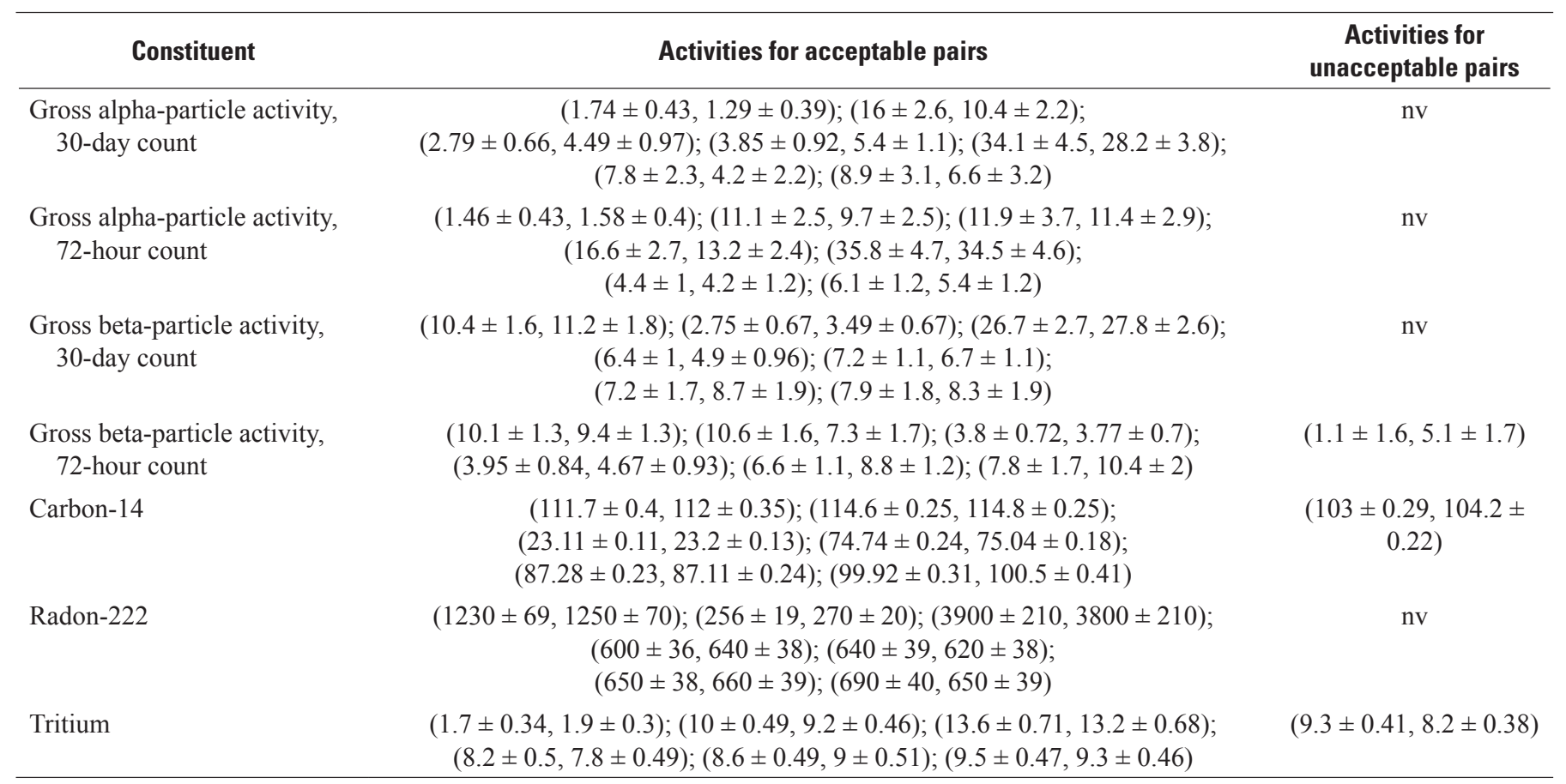

Table A-3C. Quality-control summary for replicate pair analyses of microbial indicators in groundwater samples collected from the Madera/Chowchilla-Kings Shallow Aquifer study unit, Groundwater Ambient Monitoring and Assessment (GAMA) Priority Basin Project, California, August 2013 to April 2014.

[M, detection of presence verified but not quantified; $\mathrm{U}$, not detected]

\begin{tabular}{lcccc}
\hline & $\begin{array}{c}\text { Number of } \\
\text { acceptable } \\
\text { pairs }\end{array}$ & & & $\begin{array}{c}\text { Number of } \\
\text { unacceptable } \\
\text { pairs }\end{array}$ \\
\cline { 2 - 3 } & $\mathbf{U}-\mathbf{U}$ & $\mathbf{M - M}$ & & $\mathbf{U}-\mathbf{M}$ \\
\hline Escherichia coli $($ E. coli) & 7 & 0 & & 0 \\
Total coliform & 6 & 1 & & 0 \\
Enterococci & 7 & 0 & & 0 \\
\hline
\end{tabular}


Table A-4A. Quality-control summary for matrix-spike recoveries of volatile organic compounds (VOCs) in groundwater samples collected from the Madera/Chowchilla-Kings Shallow Aquifer study unit, Groundwater Ambient Monitoring and Assessment (GAMA) Priority Basin Project, California, August 2013 to April 2014.

[Acceptable recovery range is between 70 and 130 percent. Abbreviations: na, not available; VOC, volatile organic compound]

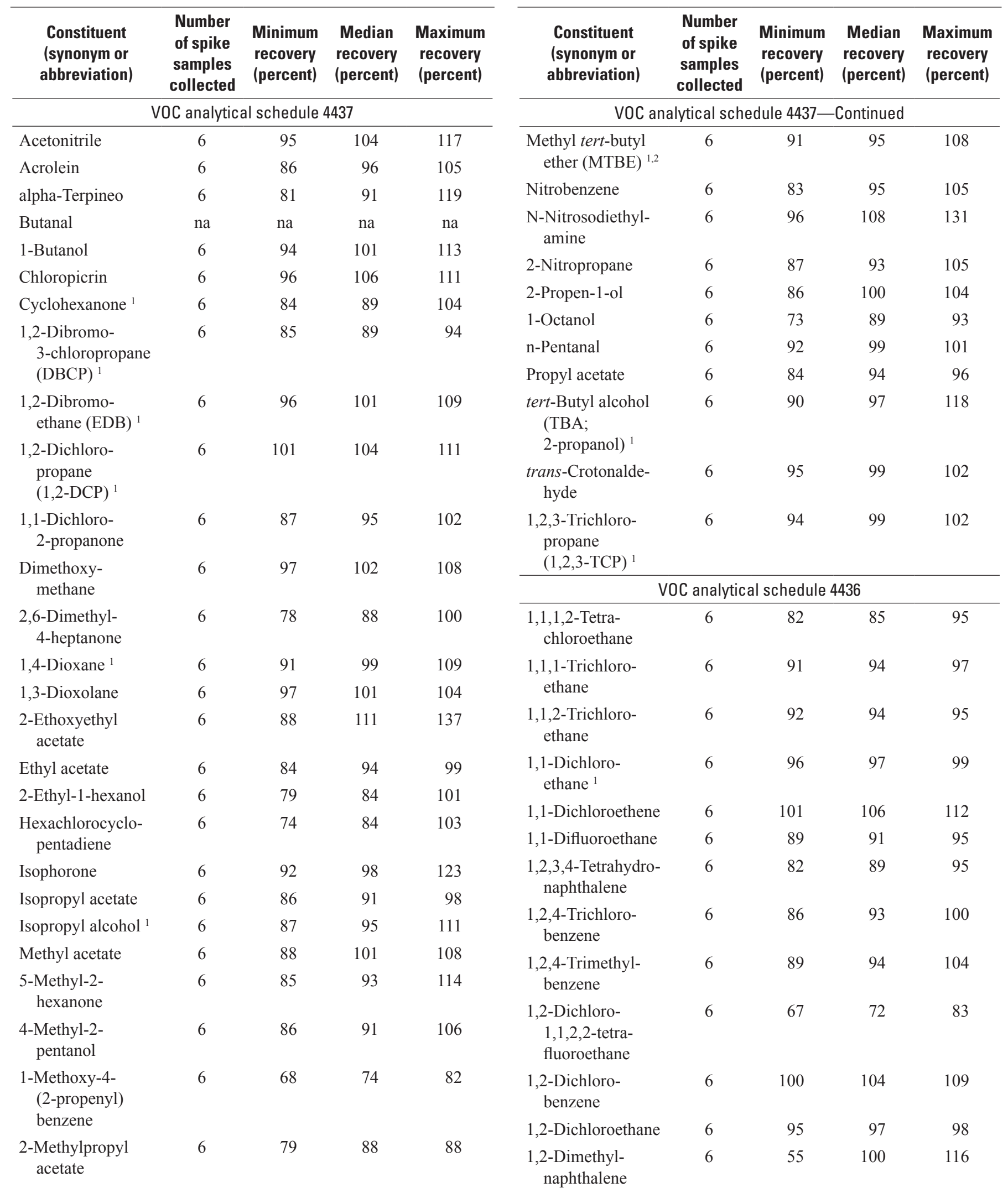


Table A-4A. Quality-control summary for matrix-spike recoveries of volatile organic compounds (VOCs) in groundwater samples collected from the Madera/Chowchilla-Kings Shallow Aquifer study unit, Groundwater Ambient Monitoring and Assessment (GAMA) Priority Basin Project, California, August 2013 to April 2014.-Continued

[Acceptable recovery range is between 70 and 130 percent. Abbreviations: na, not available; VOC, volatile organic compound]

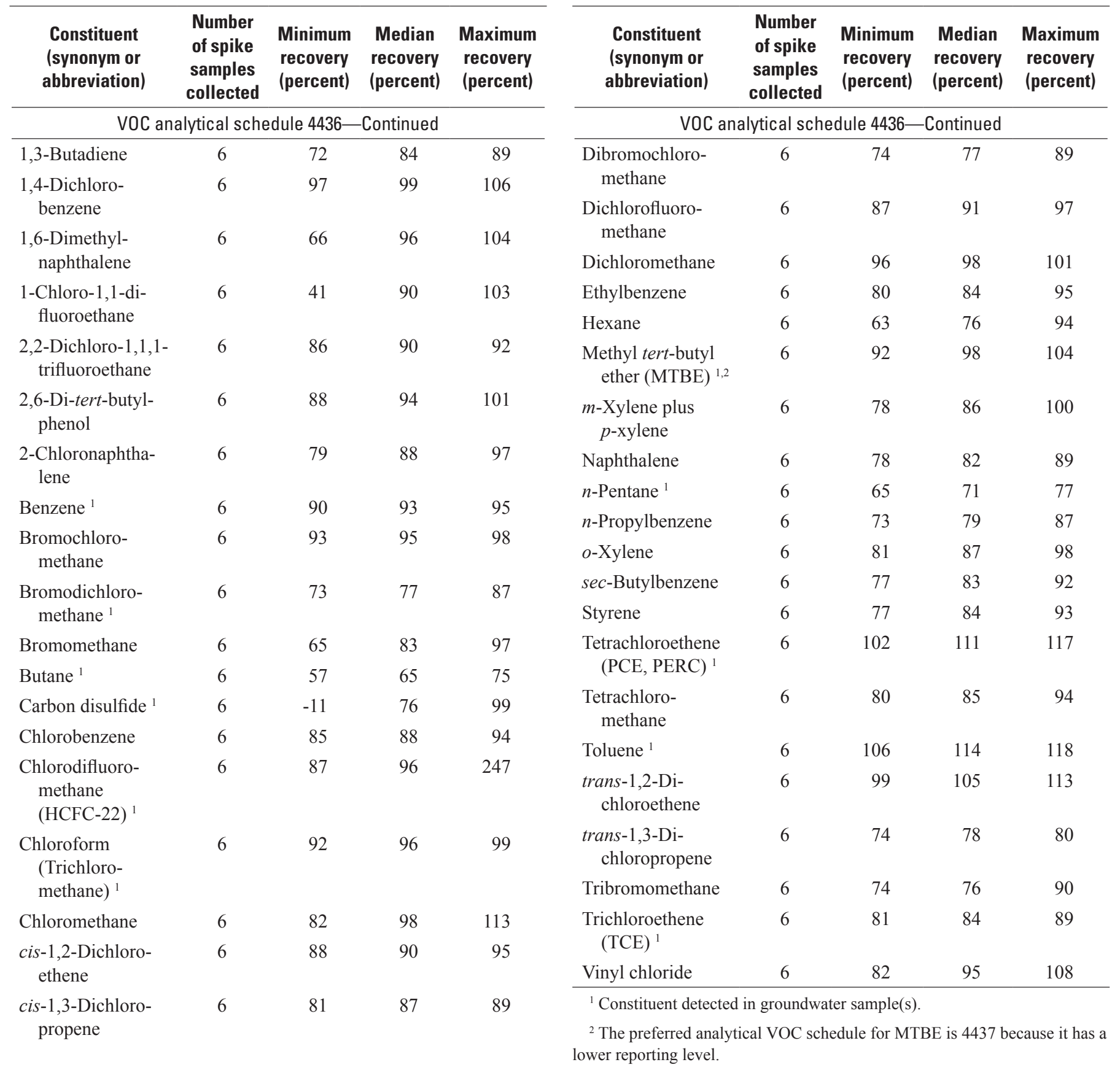


Table A-4B. Quality-control summary for matrix-spike recoveries of pesticides and pesticide degradates in samples collected for the Madera/Chowchilla-Kings Shallow Aquifer study unit, Groundwater Ambient Monitoring and Assessment (GAMA) Priority Basin Project, California, August 2013 to April 2014.

[Acceptable recovery range is between 70 and 130 percent. Abbreviations: na, not available]

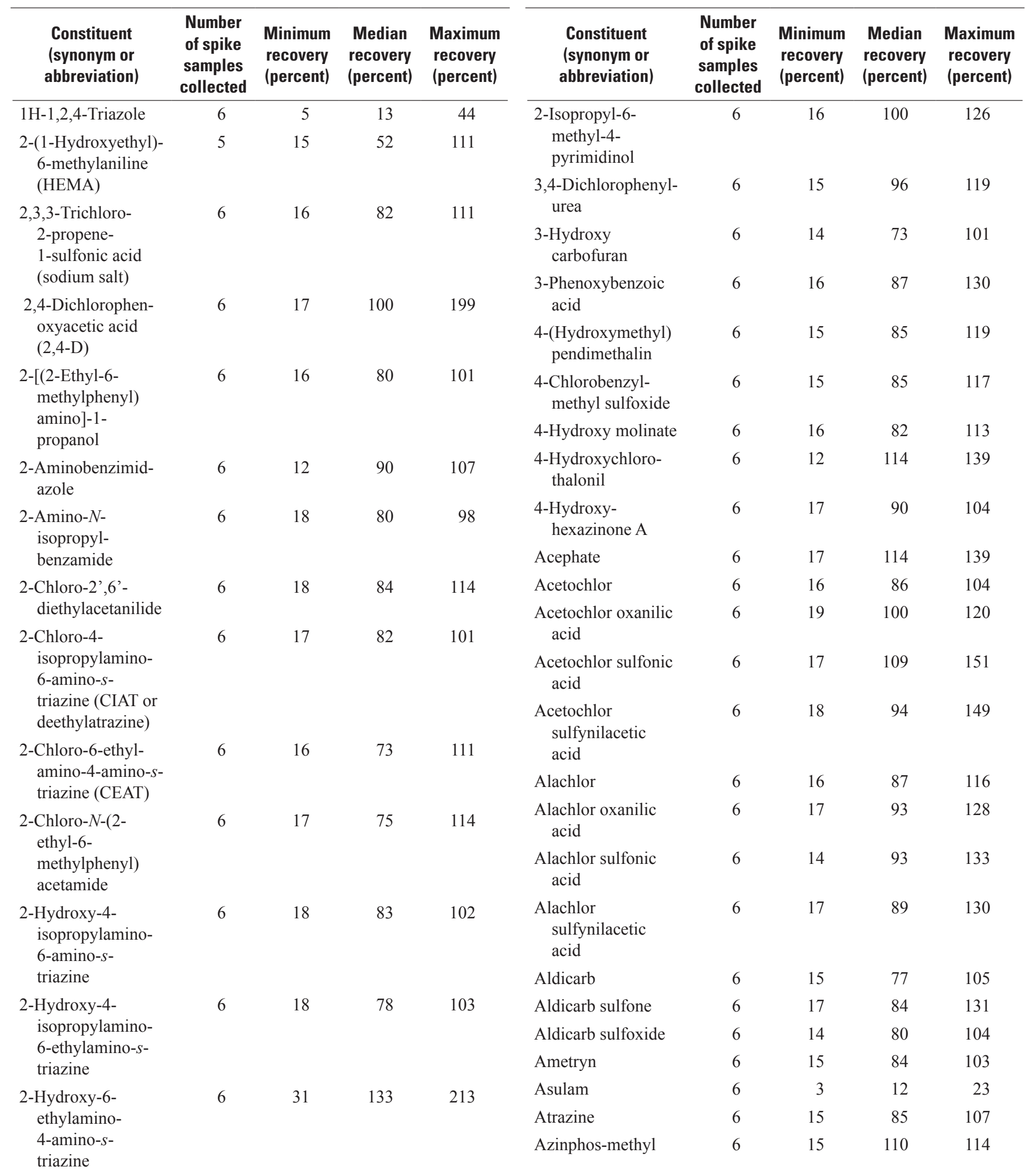


Table A-4B. Quality-control summary for matrix-spike recoveries of pesticides and pesticide degradates in samples collected for the Madera/Chowchilla-Kings Shallow Aquifer study unit, Groundwater Ambient Monitoring and Assessment (GAMA) Priority Basin Project, California, August 2013 to April 2014.-Continued

[Acceptable recovery range is between 70 and 130 percent. Abbreviations: na, not available]

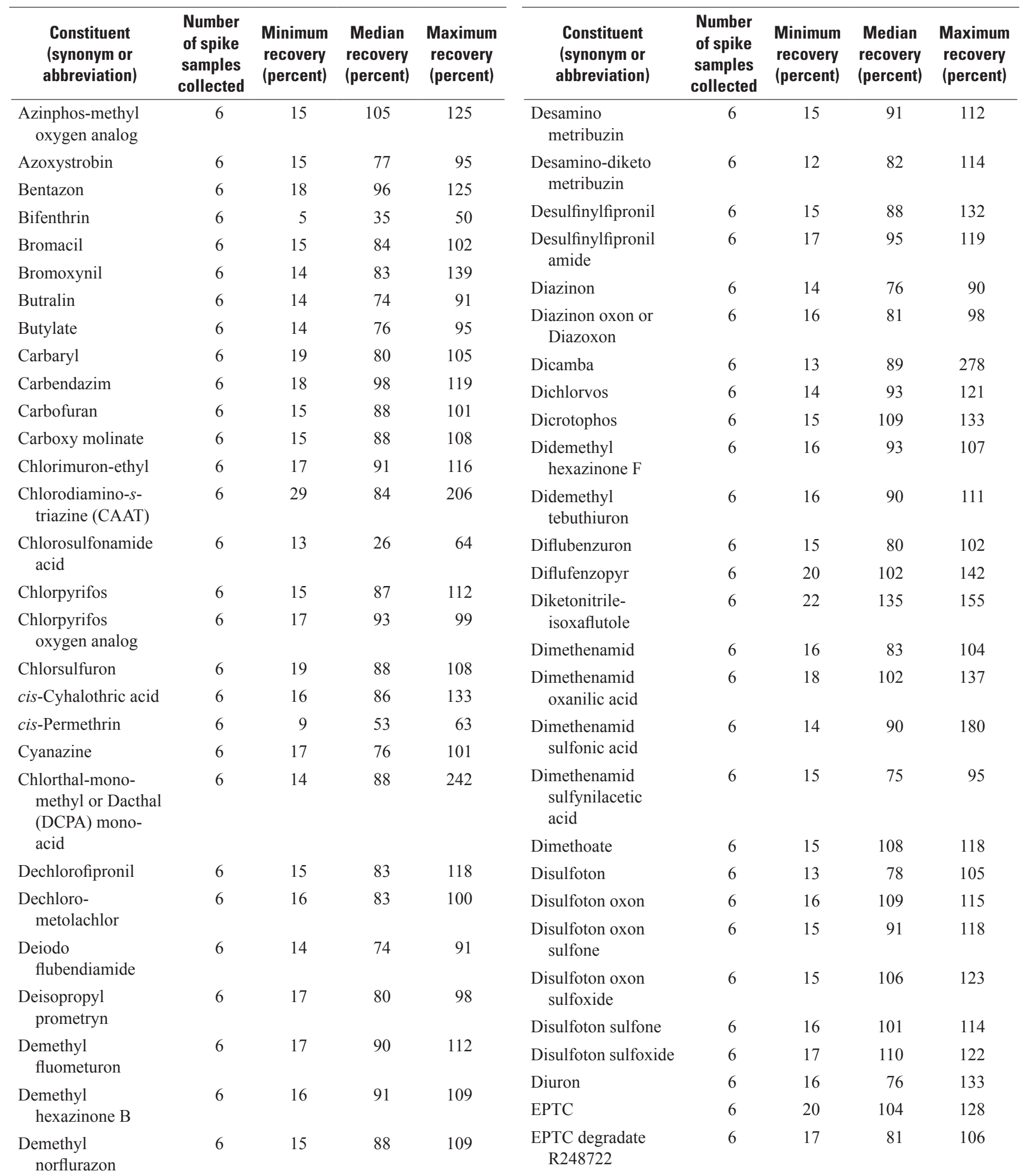


Table A-4B. Quality-control summary for matrix-spike recoveries of pesticides and pesticide degradates in samples collected for the Madera/Chowchilla-Kings Shallow Aquifer study unit, Groundwater Ambient Monitoring and Assessment (GAMA) Priority Basin Project, California, August 2013 to April 2014.-Continued

[Acceptable recovery range is between 70 and 130 percent. Abbreviations: na, not available]

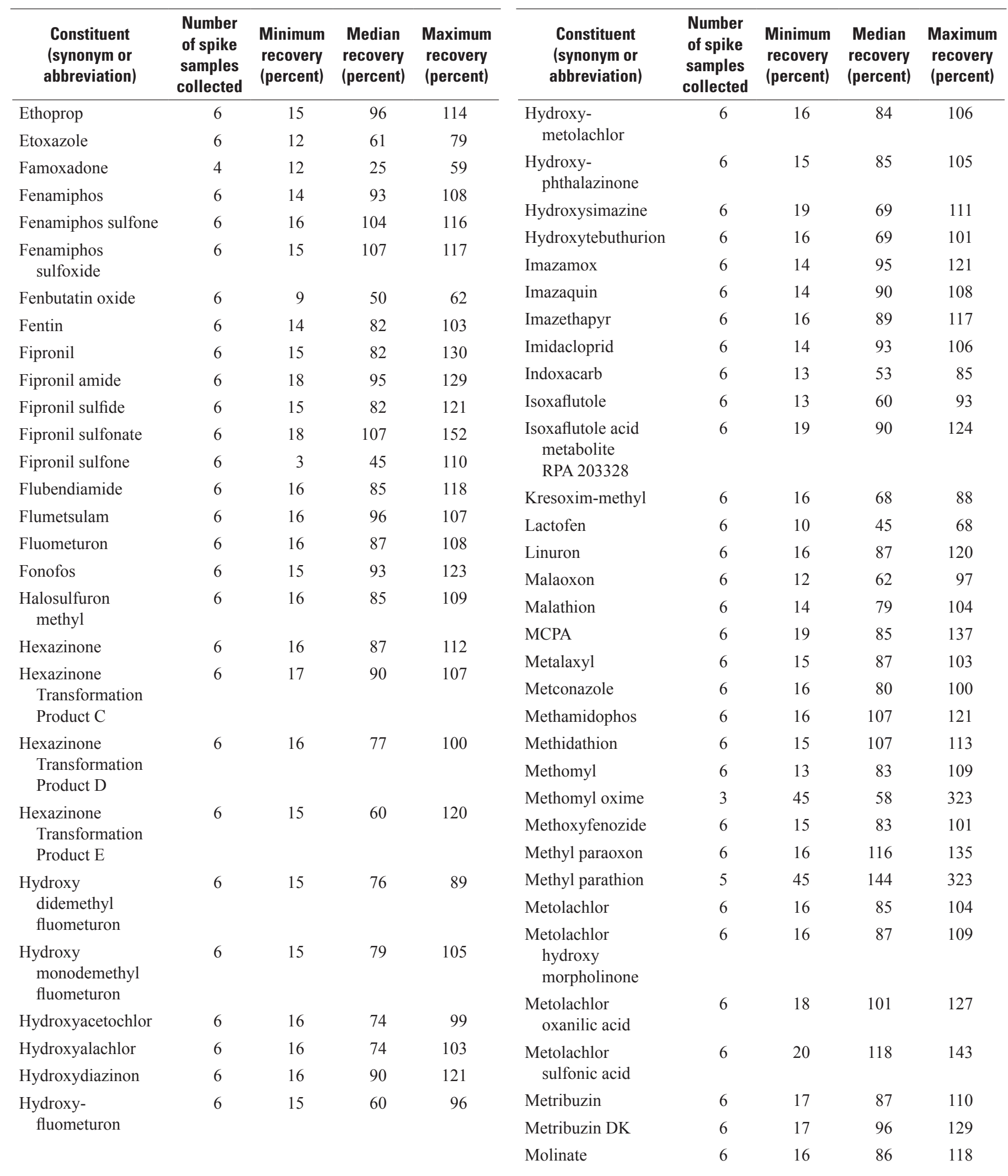


Table A-4B. Quality-control summary for matrix-spike recoveries of pesticides and pesticide degradates in samples collected for the Madera/Chowchilla-Kings Shallow Aquifer study unit, Groundwater Ambient Monitoring and Assessment (GAMA) Priority Basin Project, California, August 2013 to April 2014.-Continued

[Acceptable recovery range is between 70 and 130 percent. Abbreviations: na, not available]

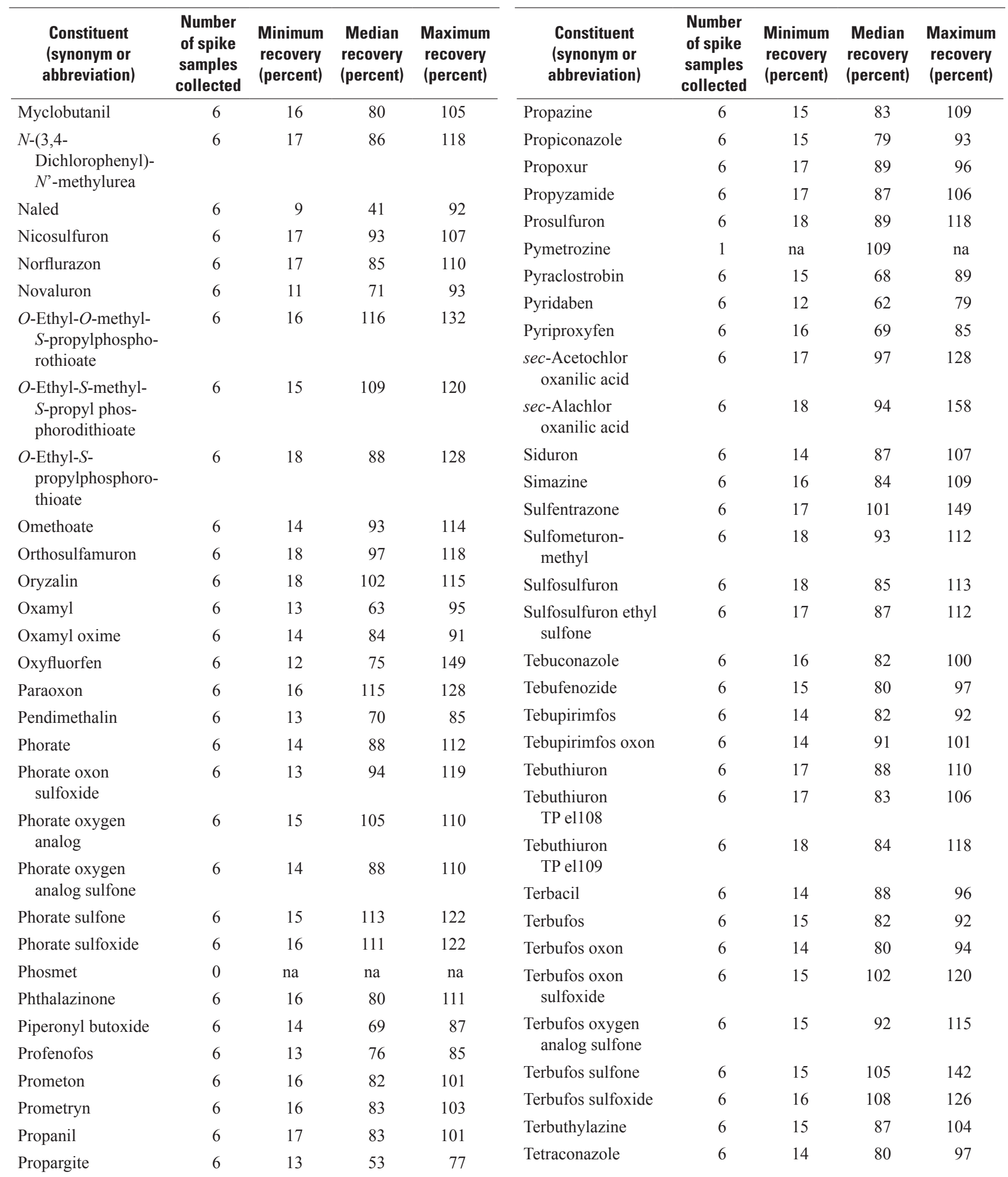


Table A-4B. Quality-control summary for matrix-spike recoveries of pesticides and pesticide degradates in samples collected for the Madera/Chowchilla-Kings Shallow Aquifer study unit, Groundwater Ambient Monitoring and Assessment (GAMA)

Priority Basin Project, California, August 2013 to April 2014.Continued

[Acceptable recovery range is between 70 and 130 percent. Abbreviations: na, not available]

\begin{tabular}{lcccc}
\hline $\begin{array}{c}\text { Constituent } \\
\text { (synonym or } \\
\text { abbreviation) }\end{array}$ & $\begin{array}{c}\text { Number } \\
\text { of spike } \\
\text { samples } \\
\text { collected }\end{array}$ & $\begin{array}{c}\text { Minimum } \\
\text { recovery } \\
\text { (percent) }\end{array}$ & $\begin{array}{c}\text { Median } \\
\text { recovery } \\
\text { (percent) }\end{array}$ & $\begin{array}{c}\text { Maximum } \\
\text { recovery } \\
\text { (percent) }\end{array}$ \\
\hline Thiobencarb & 6 & 15 & 83 & 100 \\
trans-Permethrin & 6 & 9 & 57 & 62 \\
Triallate & 6 & 16 & 82 & 99 \\
Tribuphos & 6 & 12 & 73 & 88 \\
Triclopyr & 6 & 12 & 82 & 98 \\
Tridemethyl & 6 & 17 & 80 & 106 \\
$\quad \begin{array}{l}\text { hexazinone G } \\
\text { Tridemethyl }\end{array}$ & 6 & 15 & 85 & 112 \\
tebuthiuron & & & & \\
Trifloxystrobin & 6 & 15 & 64 & 79 \\
\hline
\end{tabular}


Table A-5. Quality-control summary for surrogate compound recoveries of volatile organic compounds (VOCs) and pesticides and pesticide degradates in groundwater samples collected from the Madera/Chowchilla-Kings Shallow Aquifer study unit, Groundwater Ambient Monitoring and Assessment (GAMA) Priority Basin Project, California, August 2013 to April 2014.

[All values are in percentages.]

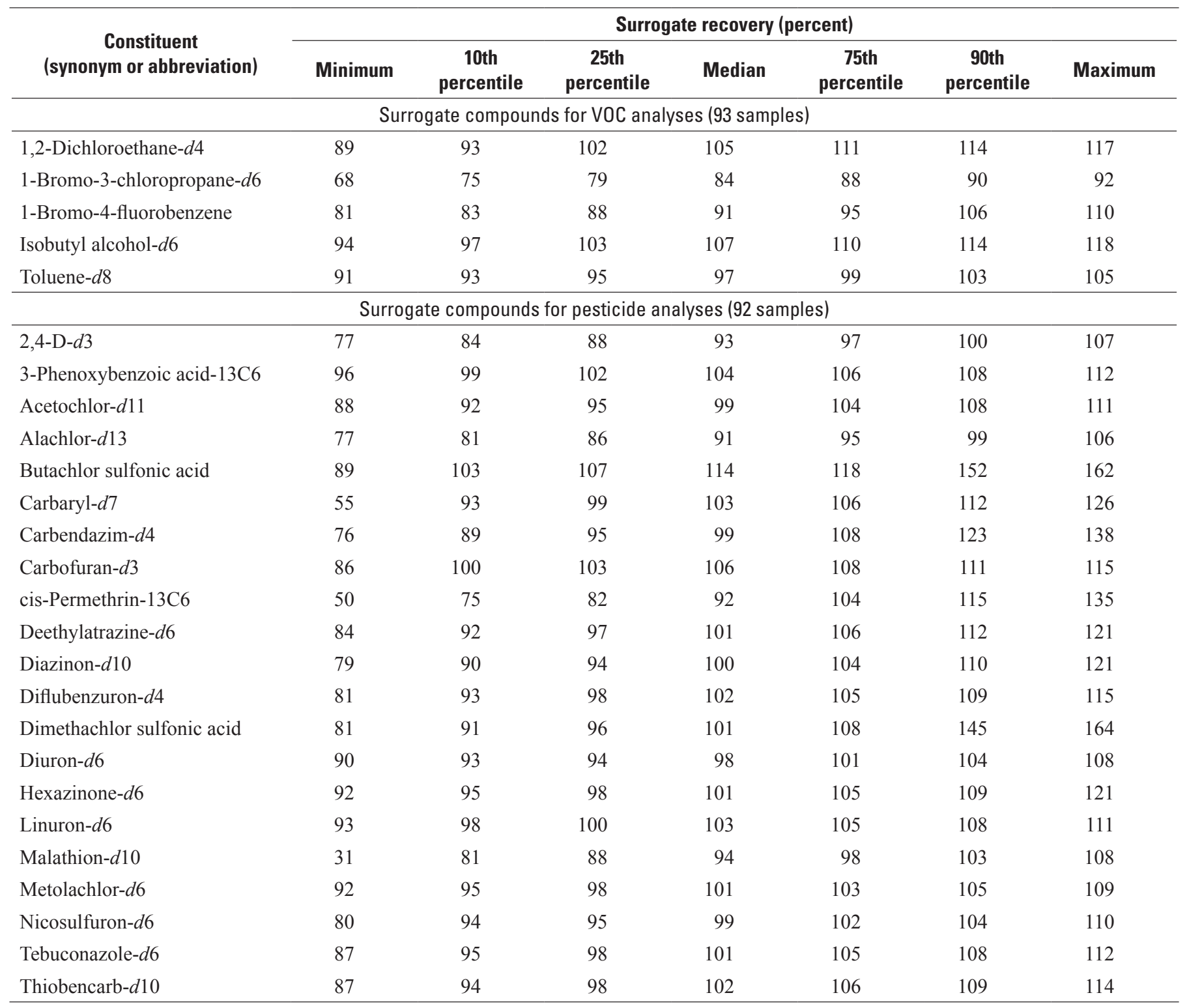



Publishing support provided by the U.S. Geological Survey Science Publishing Network, Sacramento Publishing Service Center

For more information concerning the research in this report, contact the Director, California Water Science Center

U.S. Geological Survey

$6000 \mathrm{~J}$ Street, Placer Hall

Sacramento, California 95819

http://ca.water.usgs.gov 


\section{$\frac{\mathbb{2}}{\mathbb{3}}$}

